\title{
A Phosphine-Free Manganese Catalyst Enables Selective Transfer Hydrogenation of Nitriles to Primary and Secondary Amines Using Ammonia Borane
}

Koushik Sarkar, ${ }^{\dagger a}$ Kuhali Das,${ }^{\dagger a}$ Abhishek Kundu ${ }^{\text {b }}$, Debasish Adhikari, ${ }^{\text {"b }}$ and Biplab Maji ${ }^{* a}$

aDepartment of Chemical Sciences, Indian Institute of Science Education and Research Kolkata, Mohanpur-741246 (India)

${ }^{\mathrm{b}}$ Department of Chemical Sciences, Indian Institute of Science Education and Research Mohali, SAS Nagar140306 (India)

Email: adhikari@iisermohali.ac.in,bm@iiserkol.ac.in

Table of Contents

1. General information

2. Synthesis of the ligands and metal complexes

2a. Synthesis of the ligands

2b. Synthesis of the metal complexes

3.Detailed reaction optimization tables of primary amine (2) synthesis

4. Detailed reaction optimization tables of symmetrical secondary amine (3) synthesis

5. Detailed reaction optimization tables of asymmetrical secondary amine (4) synthesis

6. Mechanistic studies

6a. IR analysis

6b. ${ }^{11}$ B NMR experiment at different time intervals

6c. Isotope labeling experiment

6d. Control experiment

7. Kinetic experiments

7a. Determination of reaction order for $\mathrm{Mn} 2$ in toluene

$7 \mathrm{~b}$. Determination of reaction order for $1 \mathrm{a}$ in toluene

7c. Determination of reaction order for $\mathrm{NH}_{3} \mathrm{BH}_{3}$ in toluene

7d. Determination of reaction order for Mn2 in THF

7e. Determination of reaction order for $\mathrm{NH}_{3} \mathrm{BH}_{3}$ in $\mathrm{THF}$

7f. Determination of reaction order for $1 \mathrm{a}$ in THF

7g. Eyring analysis

7h. Determination of kinetic isotope effect (KIE)

7i. Probing the dehydrogenation of $\mathrm{NH}_{3} \mathrm{BH}_{3}$ 


\section{Analytical data for the synthesized compounds}

\section{Computational details}

\section{Copies of the IR spectrum of metal complexes}

\section{Copies of GCMS traces}

\section{Copies of the ${ }^{1} \mathrm{H}$ and ${ }^{13} \mathrm{C}$ spectrum}

\section{References}

\section{General information:}

All the reactions were conducted under the $\mathrm{Ar}$ and $\mathrm{N}_{2}$ atmosphere. The glasswares were dried in an oven. Solvents were dried under the standard protocol. All the solvents were degassed and stored over activated molecular sieves ( $4 \AA$ ). The chemicals were purchased from Avra Synthesis, Sigma-Aldrich, TCI, Alfa Aeser and used without further purification unless otherwise mentioned. Ligands and metal complexes were prepared according to our published procedures. ${ }^{1}{ }^{1} \mathrm{H},{ }^{13} \mathrm{C},{ }^{19} \mathrm{~F},{ }^{11} \mathrm{~B}$, NMR spectra were collected using BRUKER $\left({ }^{1} \mathrm{H}: 500 \mathrm{MHz},{ }^{13} \mathrm{C}: 126 \mathrm{MHz}\right)$ and JEOL $\left({ }^{1} \mathrm{H}: 400 \mathrm{MHz},{ }^{13} \mathrm{C}: 100 \mathrm{MHz}\right)$ instrument. NMR data was taken in the ppm unit and referenced against the solvent residual peaks. Coupling constants $(\mathrm{J})$ are reported in Hertz $(\mathrm{Hz})$. Coupling patterns are indicated as s (singlet), d (doublet), t (triplet), dd (doublet of doublet), td (triplet of doublet), or m (multiplet). FT-IR spectra were recorded by BRUKER FT-IR Spectrometer. The elemental analyses were carried out with a PerkinElmer 240C elemental analyzer. Mass spectral analyses were done in Bruker micrOTOF-Q II Spectrometer. For thin-layer chromatography (TLC) analysis, Merck precoated TLC plates (silica gel $60 \mathrm{~F} 254 / 0.25 \mathrm{~mm})$ were used. Visualization was accomplished by UV light $(254 \mathrm{~nm})$. All the kinetics study has been done by using gas chromatography using appropriate internal standard.

\section{Synthesis of the ligands and metal complexes}

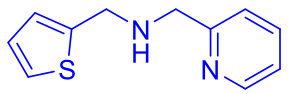

L1

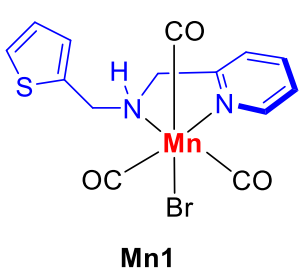

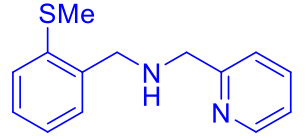

L2

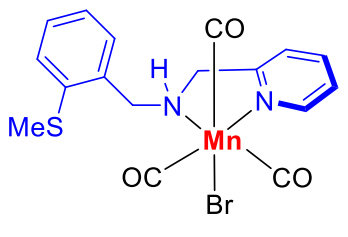

Mn2

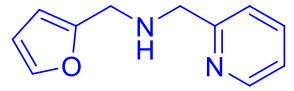

L3

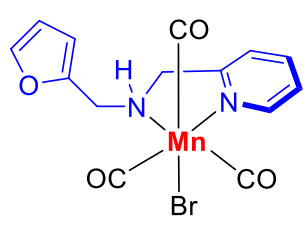

Mn3

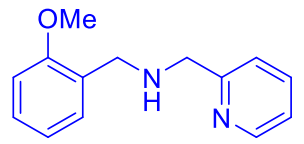

L4

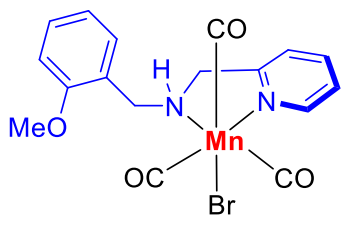

Mn4

Figure S1. Ligands L1-4 and Mn complexes Mn1-4.

\section{2a. Synthesis of ligands}

Ligands were synthesized according to the literature procedure published by our group. ${ }^{\text {a }}$

Synthesis of $\mathbf{L} 1$ and $\mathbf{L 3}^{1}$ : The corresponding aldehyde $(2 \mathrm{mmol})$ and 2-(aminomethyl)pyridine (2.2 mmol) were mixed and stirred at room temperature in methanol $(20 \mathrm{~mL})$ in the presence of excess $\mathrm{Na}_{2} \mathrm{SO}_{4}(5 \mathrm{mmol})$ for $5 \mathrm{~h}$. Filtration was done to remove the solid particles from the reaction mixture. After adding $\mathrm{NaBH}_{4}(2 \mathrm{mmol})$ at $0{ }^{\circ} \mathrm{C}$, the mixture was gently warmed at $45^{\circ} \mathrm{C}$ for $1 \mathrm{~h}$. The solvent was then removed under reduced pressure, and water $(5 \mathrm{~mL})$ was then added. The organic part was extracted with DCM and washed with brine. All the volatiles were then evaporated, and the residue was purified by column chromatography on silica gel.

Pyridin-2-yl-N-(thiophen-2-ylmethyl)methanamine (L1): Yield = 85\%. (350 mg, $1.7 \mathrm{mmol}$ ). ${ }^{1} \mathbf{H}$ NMR $\left(400 \mathrm{MHz}, \mathrm{CDCl}_{3}\right) \delta 8.52(\mathrm{~d}, \mathrm{~J}=4.3 \mathrm{~Hz}, 1 \mathrm{H}), 7.61(\mathrm{td}, \mathrm{J}=7.7,1.6 \mathrm{~Hz}, 1 \mathrm{H}), 7.29(\mathrm{~d}, \mathrm{~J}=7.7$ 
$\mathrm{Hz}, 1 \mathrm{H}), 7.18(\mathrm{dd}, \mathrm{J}=4.4,2.1 \mathrm{~Hz}, 1 \mathrm{H}), 7.13(\mathrm{dd}, \mathrm{J}=7.4,4.8 \mathrm{~Hz}, 1 \mathrm{H}), 6.95-6.89(\mathrm{~m}, 2 \mathrm{H}), 4.01(\mathrm{~s}, 2 \mathrm{H})$, $3.92(\mathrm{~s}, 2 \mathrm{H}), 2.13(\mathrm{~s}, 1 \mathrm{H})$.

1-(furan-2-yl)-N-(3yridine-2-ylmethyl)methanamine (L3): Yield $=50 \%(190 \mathrm{mg}, 1 \mathrm{mmol}) .{ }^{1} \mathbf{H}$ NMR $\left(400 \mathrm{MHz}, \mathrm{CDCl}_{3}\right) \delta 8.55(\mathrm{dd}, \mathrm{J}=4.8,1.2 \mathrm{~Hz}, 1 \mathrm{H}), 7.63(\mathrm{td}, \mathrm{J}=7.7,1.8 \mathrm{~Hz}, 1 \mathrm{H}), 7.35(\mathrm{~d}, \mathrm{~J}=$ $1.6 \mathrm{~Hz}, 1 \mathrm{H}), 7.30(\mathrm{~d}, \mathrm{~J}=7.8 \mathrm{~Hz}, 1 \mathrm{H}), 7.15(\mathrm{dd}, \mathrm{J}=7.7,4.9 \mathrm{~Hz}, 1 \mathrm{H}), 6.30(\mathrm{dd}, \mathrm{J}=3.1,2.0 \mathrm{~Hz}, 1 \mathrm{H}), 6.19$ $(\mathrm{d}, \mathrm{J}=2.9 \mathrm{~Hz}, 1 \mathrm{H}), 3.92(\mathrm{~s}, 2 \mathrm{H}), 3.83(\mathrm{~s}, 2 \mathrm{H}), 2.71(\mathrm{~s}, 1 \mathrm{H}) .{ }^{13} \mathbf{C} \mathbf{N M R}\left(101 \mathrm{MHz}, \mathrm{CDCl}_{3}\right) \delta 159.5,153.8$, $149.4,142.0,136.6,122.5,122.1,110.2,107.3,54.3,45.8$.

Synthesis of L2: In a $50 \mathrm{~mL}$ round bottom Schlenk flask, $20 \mathrm{~mL}$ DMF and sodium thiomethoxide (4.7 mmol) were taken under Ar. Then bromobenzaldehyde $(2 \mathrm{mmol})$ was added dropwise, and the reaction mixture was stirred for 15 mins at room temperature. Next, $60 \mathrm{~mL} 1 \mathrm{M} \mathrm{HCl}$ was added to the reaction mixture, and the organic layer was extracted with DCM $(3 \times 60 \mathrm{~mL})$. The organic part was washed with water $(4 \times 20 \mathrm{~mL})$. The product 2-(methylthio)benzaldehyde was purified by column chromatography. After that, 2-picolylamine ( $2.2 \mathrm{mmol}$ ) was added in a solution of 2-(methylthio)benzaldehyde ( $2 \mathrm{mmol}$ ) in methanol $(20 \mathrm{~mL})$, followed by the addition of $\mathrm{Na}_{2} \mathrm{SO}_{4}(4 \mathrm{mmol})$ under Ar. The reaction was stirred for $5 \mathrm{~h}$ at $37{ }^{\circ} \mathrm{C}$. The reaction mixture was filtered, and to the filtrate, $\mathrm{NaBH}_{4}(2 \mathrm{mmol})$ was added, keeping the temperature at $0{ }^{\circ} \mathrm{C}$. The mixture was then warmed at $45^{\circ} \mathrm{C}$ and stirred for $1 \mathrm{~h}$. After that, all volatiles were removed by reduced pressure, and $5 \mathrm{~mL}$ of water was added. The organic part was extracted with DCM and washed with brine. The obtained crude organic part was purified using column chromatography to afford the product (L2).

N-(2-(methylthio)benzyl)-1-(3yridine-2-yl)methanamine (L2): ${ }^{\text {b, } 2}$ Yield $=80 \%(390 \mathrm{mg}, 1.6$ mmol). ${ }^{1} \mathbf{H}$ NMR $\left(400 \mathrm{MHz}, \mathrm{CDCl}_{3}\right) \delta 8.55(\mathrm{~d}, J=3.8 \mathrm{~Hz}, 1 \mathrm{H}), 7.67-7.60(\mathrm{~m}, 1 \mathrm{H}), 7.36(\mathrm{t}, J=8.1 \mathrm{~Hz}$, 2H), $7.26-7.20(\mathrm{~m}, 2 \mathrm{H}), 7.18-7.08(\mathrm{~m}, 2 \mathrm{H}), 3.95(\mathrm{~s}, 2 \mathrm{H}), 3.92(\mathrm{~s}, 2 \mathrm{H}), 2.47$ (s, 3H), 2.04 (br s, 1H). ${ }^{13} \mathrm{C}$ NMR $\left(101 \mathrm{MHz}, \mathrm{CDCl}_{3}\right) \delta 160.1,149.4,137.9,137.7,136.5,129.1,127.9$ 125.8, 125.0, 122.4, $122.0,54.8,51.5,15.9$.

Synthesis of L4: ${ }^{3}$ Pyridine-2-carbaldehyde ( $2 \mathrm{mmol}$ ) and (2-methoxyphenyl)methanamine ( $\left.2.5 \mathrm{mmol}\right)$ were taken in a flask containing $20 \mathrm{~mL}$ of methanol. Two equivalents of $\mathrm{Na}_{2} \mathrm{SO}_{4}$ was added to it. The resulting solution was stirred at r.t. for $5 \mathrm{~h}$. Then the solution was filtered, and to the filtrate, $\mathrm{NaBH}_{4}(2$ mmol) was added, keeping the temperature at $0{ }^{\circ} \mathrm{C}$. The mixture was warmed at $45{ }^{\circ} \mathrm{C}$ and stirred for 1 $\mathrm{h}$. The solvent was removed under reduced pressure. Water $(5 \mathrm{~mL})$ was added, and the organic part was extracted with DCM. The DCM layer was washed with brine and dried over anhydrous $\mathrm{Na}_{2} \mathrm{SO}_{4}$. The obtained crude organic part was purified using column chromatography to afford the product $(\mathbf{L 4})$.

N-(2-methoxybenzyl)-1-(3yridine-2-yl)methanamine (L4): Yield = 82\% (373.9 mg, $1.64 \mathrm{mmol}) .{ }^{\mathbf{1}} \mathbf{H}$ NMR $\left(400 \mathrm{MHz}, \mathrm{CDCl}_{3}\right) \delta 8.54(\mathrm{~d}, J=4.8 \mathrm{~Hz}, 1 \mathrm{H}), 7.63(\mathrm{td}, J=7.8,1.6 \mathrm{~Hz}, 1 \mathrm{H}), 7.36(\mathrm{~d}, J=7.8 \mathrm{~Hz}$, $1 \mathrm{H}), 7.28(\mathrm{~d}, J=7.3 \mathrm{~Hz}, 1 \mathrm{H}), 7.23(\mathrm{td}, J=8.0,1.3 \mathrm{~Hz}, 1 \mathrm{H}), 7.14(\mathrm{dd}, J=6.9,5.3 \mathrm{~Hz}, 1 \mathrm{H}), 6.91(\mathrm{t}, J=$ $7.7 \mathrm{~Hz}, 1 \mathrm{H}), 6.86(\mathrm{~d}, J=8.1 \mathrm{~Hz}, 1 \mathrm{H}), 3.92(\mathrm{~s}, 2 \mathrm{H}), 3.86(\mathrm{~s}, 2 \mathrm{H}), 3.83(\mathrm{~s}, 3 \mathrm{H}) .{ }^{13} \mathbf{C} \mathbf{~ N M R}(101 \mathrm{MHz}$, $\left.\mathrm{CDCl}_{3}\right) \delta 160.3,157.8,149.3,136.5,130.0,128.4,122.3,121.9,120.5,110.3,55.4,54.8,49.0$.

\section{2b. Synthesis of the metal complex Mn1-4:}

Synthesis of Mn1-3: The Mn1-3 complexes were synthesized according to our previously published procedure. ${ }^{1}$

In a $10 \mathrm{~mL}$ Schlenk tube, the corresponding ligand $(0.2 \mathrm{mmol})$ and $\mathrm{Mn}(\mathrm{CO})_{5} \mathrm{Br}(0.22 \mathrm{mmol})$ were added in $4 \mathrm{~mL}$ THF under $\mathrm{Ar}$, and the reaction was stirred at $80{ }^{\circ} \mathrm{C}$ for $3 \mathrm{~h}$. After that, the THF was evaporated under the vacuum, and the residue was washed with hexane $(3 \times 2 \mathrm{~mL})$. The corresponding Metal complexes were obtained as yellow to brown colored solid.

Mn1. Yield = 93\% (80 mg, 0.18 mmol). Selected IR (ATR, $\left.v \mathrm{~cm}^{-1}\right): 3182.3,2019.7$ (s, vco), 1938.1 (s, vco), 1865.3 (s, vco), 1619.9, 1462.9, 1271.1, 1120.2, 1026.4, 965.2, 791.8, 706.1. 
Mn2. Yield $=90 \%(84 \mathrm{mg}, 0.18 \mathrm{mmol})$. Selected IR (ATR, $\left.v \mathrm{~cm}^{-1}\right): 3199,2028\left(\mathrm{~s}, v_{\mathrm{CO}}\right), 1940\left(\mathrm{~s}, v_{\mathrm{CO}}\right)$, $1910\left(\mathrm{~s}, v_{\mathrm{CO}}\right), 1453,1279,1108,996,796,782,653,549$.

Mn3. Yield $=84 \%$ (69 mg, $0.17 \mathrm{mmol}$ ). Selected IR (ATR, $\left.v \mathrm{~cm}^{-1}\right)$ : 3182.3, 2021.7 (s, vco), 1936.1 (s, $v \mathrm{co}$ ), 1897.3 (s, vco), 1617.9, 1479.5, 1464.9, 1166.1, 1030.5, 971.3, 789.8, 751.0, 706.1, 651.1.

Synthesis of Mn4: In a $10 \mathrm{~mL}$ Schlenk tube, $\mathbf{L 4}(0.2 \mathrm{mmol})$, and $\mathrm{Mn}(\mathrm{CO})_{5} \mathrm{Br}(0.22 \mathrm{mmol})$ were added in $4 \mathrm{~mL}$ THF under Ar, and the reaction was stirred at $80^{\circ} \mathrm{C}$ for $3 \mathrm{~h}$. After that, the THF was evaporated under vacuum, and the yellow-colored residue was washed with $n$-hexane $(3 \times 2 \mathrm{~mL})$. Mn4 was obtained as yellow solid and characterized by IR, ${ }^{1} \mathrm{H},{ }^{13} \mathrm{C}$ NMR, and HRMS analysis. Yield $=90 \%(80$ $\mathrm{mg}, 0.18 \mathrm{mmol})$.

Selected IR (ATR, $\left.v \mathrm{~cm}^{-1}\right)$ : 3227, $2024\left(v_{\mathrm{CO}}\right), 1908\left(v_{\mathrm{CO}}\right), 1614,1459,1257,1044,1000,780,653 .{ }^{1} \mathbf{H}$ NMR $\left(500 \mathrm{MHz}, \mathrm{CDCl}_{3}\right) \delta 9.02(\mathrm{~s}, 1 \mathrm{H}), 7.72(\mathrm{~s}, 1 \mathrm{H}), 7.53-7.09(\mathrm{~m}, 4 \mathrm{H}), 6.99(\mathrm{~s}, 1 \mathrm{H}), 4.89(\mathrm{~s}, 2 \mathrm{H})$, $4.71(\mathrm{~s}, 2 \mathrm{H}), 3.91(\mathrm{~s}, 3 \mathrm{H}) .{ }^{13} \mathbf{C} \mathbf{~ N M R}\left(126 \mathrm{MHz}, \mathrm{CDCl}_{3}\right) \delta 221.4,159.4,158.1,154.0,138.2,130.9$, 130.7, 124.8, 124.1, 121.4, 120.9, 110.9, 57.6, 57.4, 55.6. HRMS: Calculated for $\left[\mathrm{C}_{17} \mathrm{H}_{16} \mathrm{MnN}_{2} \mathrm{O}_{4}\right.$; $[\mathrm{M}$ $\left.-\mathrm{Br}]^{+}\right]$367.0491, found 367.0487.

\section{Detailed reaction optimization for primary amine (2) synthesis.}

\section{General procedure.}

In a $15 \mathrm{~mL}$ reaction tube $\mathrm{Mn} 2$ ( $3 \mathrm{~mol} \%, 3.5 \mathrm{mg})$ and $\mathrm{NH}_{3} \mathrm{BH}_{3}(0.75 \mathrm{mmol}, 23.2 \mathrm{mg})$ were added under Ar followed by addition of $n$-hexane $(0.25 \mathrm{~mL})$. After that, $1 \mathbf{a}(0.25 \mathrm{mmol}, 25.8 \mu \mathrm{L})$ was added, and the reaction tube was sealed. The tube was placed in a preheated oil bath at $60{ }^{\circ} \mathrm{C}$ and stirred for $6 \mathrm{~h}$. Then, the reaction mixture was cooled at room temperature and quenched by the addition of methanol $(1 \mathrm{~mL})$. Mesitylene $(0.25 \mathrm{mmol}, 35 \mu \mathrm{L})$ was added as an internal standard, and yield was determined by GC-MS analysis. After that, the reaction mixture was filtered through the celite bed, and then 0.5 $\mathrm{mL} \mathrm{HCl} \mathrm{(1M} \mathrm{in} \mathrm{ether)} \mathrm{was} \mathrm{added} \mathrm{dropwise} \mathrm{into} \mathrm{the} \mathrm{filtrate,} \mathrm{and} \mathrm{quick} \mathrm{precipitation} \mathrm{was} \mathrm{observed.} \mathrm{The}$ precipitate was washed with $n$-hexane and EtOAc and dried under the vacuum. The product was characterized by ${ }^{1} \mathrm{H}$ and ${ }^{13} \mathrm{C}$ NMR spectroscopy using $\mathrm{D}_{2} \mathrm{O}$ as the solvent.

\section{Table S1: Screening of catalyst.}

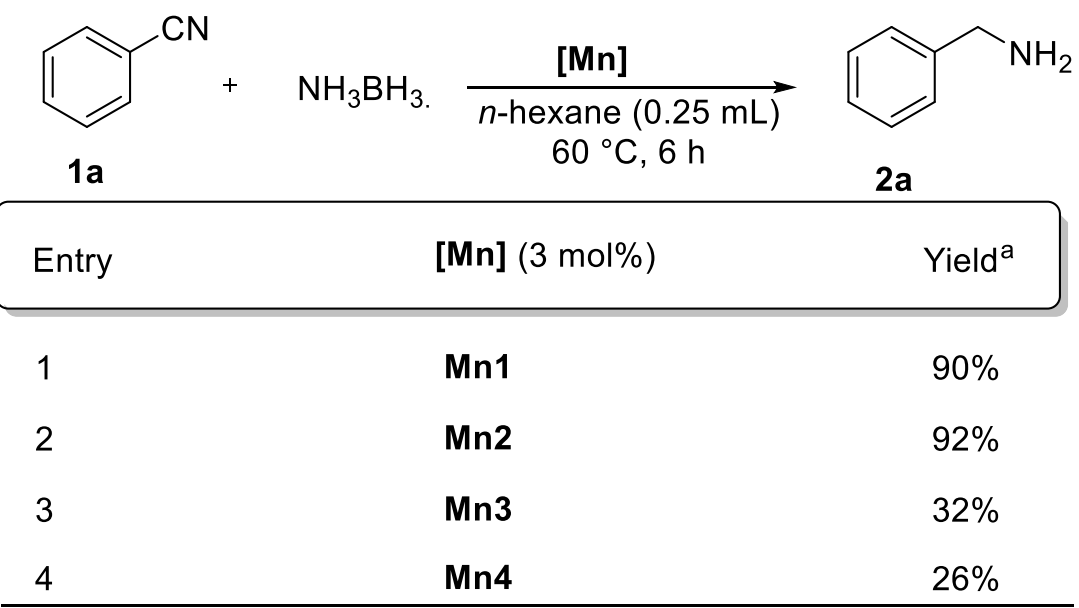

Reaction condition: $1 \mathbf{a}(0.25 \mathrm{mmol}),[\mathrm{Mn}]$ (3 mol\%), $\mathrm{NH}_{3} \mathrm{BH}_{3}(0.75 \mathrm{mmol}), n$-hexane $(0.25 \mathrm{~mL}), 60$ ${ }^{\circ} \mathrm{C}$, Ar, 6 h. ${ }^{\text {a } Y i e l d ~ w a s ~ o b t a i n e d ~ b y ~ G C-M S ~ a n a l y s i s ~ u s i n g ~ m e s i t y l e n e ~}(0.25 \mathrm{mmol})$ as an internal standard. 
Table S2: Screening of $\mathrm{NH}_{3} \mathrm{BH}_{3}$.

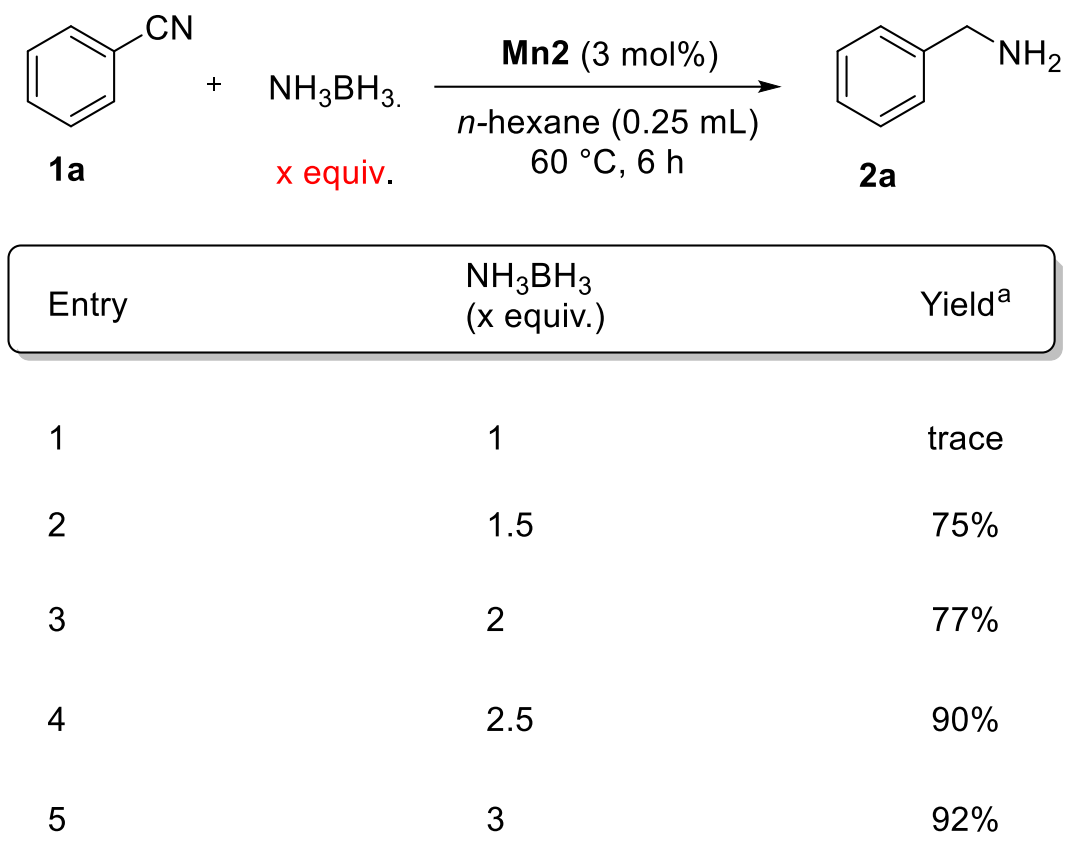

Reaction condition: 1a $(0.25 \mathrm{mmol}), \mathbf{M n} 2$ ( $3 \mathrm{~mol} \%), \mathrm{NH}_{3} \mathrm{BH}_{3}$ (x equiv.), $n$-hexane $(0.25 \mathrm{~mL}), 60^{\circ} \mathrm{C}$, Ar, 6 h. ${ }^{a}$ Yield was obtained by GC-MS analysis using mesitylene $(0.25 \mathrm{mmol})$ as an internal standard.

Table S3: Screening of temperature.

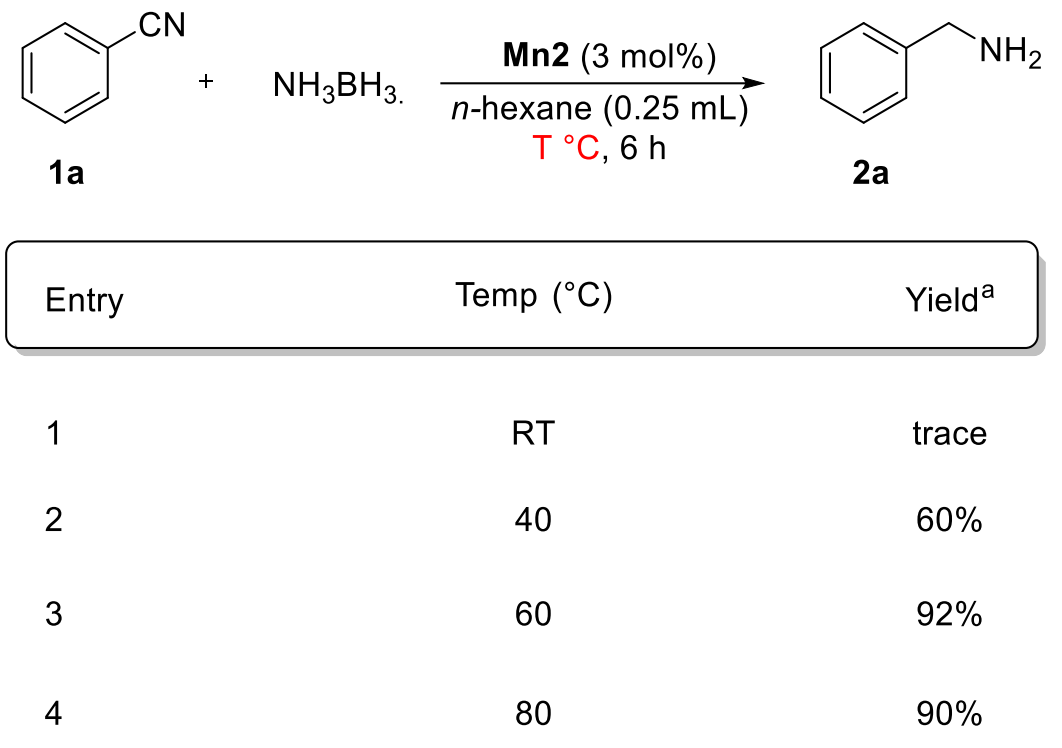

Reaction condition: $1 \mathrm{a}(0.25 \mathrm{mmol}), \mathrm{Mn} 2(3 \mathrm{~mol} \%), \mathrm{NH}_{3} \mathrm{BH}_{3}(0.75 \mathrm{mmol}), n$-hexane $(0.25 \mathrm{~mL}), \mathrm{T}$

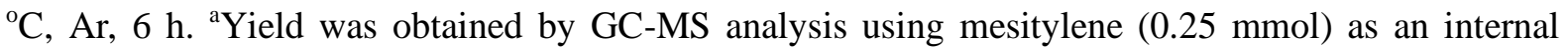
standard. 
Table S4: Screening of solvent.

\begin{tabular}{|ccc|}
\hline Entry & $\mathrm{NH}_{3} \mathrm{BH}_{3}$ & $\begin{array}{c}\text { Solvent, } \\
6{ }^{\circ} \mathrm{C}, 6 \mathrm{~h}\end{array}$ \\
\hline 1. & Solvent & Yal \\
2. & $n$-haxane & $\mathrm{NH}_{2}$ \\
3. & toluene & $92 \%$ \\
4. & $\mathrm{THF}$ & $90 \%$ \\
5. & DCM & $90 \%$ \\
\hline
\end{tabular}

Reaction condition: 1a $(0.25 \mathrm{mmol})$, $\mathrm{Mn} 2$ (3 mol\%), $\mathrm{NH}_{3} \mathrm{BH}_{3}(0.75 \mathrm{mmol})$, solvent $(0.25 \mathrm{~mL}), 60^{\circ} \mathrm{C}$, Ar, 6 h. ${ }^{\text {a}}$ Yield was obtained by GC-MS analysis using mesitylene $(0.25 \mathrm{mmol})$ as an internal standard.

\section{Detailed reaction optimization for symmetrical secondary amine (3) synthesis}

\section{General procedure.}

In a $15 \mathrm{~mL}$ reaction tube $\mathrm{Mn1}(3 \mathrm{~mol} \%, 3.5 \mathrm{mg})$ and $\mathrm{NH}_{3} \mathrm{BH}_{3}(0.75 \mathrm{mmol}, 23.2 \mathrm{mg}$ ) were added under Ar followed by the addition of $i$-PrOH $(0.5 \mathrm{~mL})$ and $1 \mathbf{a}(0.25 \mathrm{mmol}, 25.8 \mu \mathrm{L})$. The reaction tube was sealed and placed in a preheated oil bath at $60{ }^{\circ} \mathrm{C}$ for $12 \mathrm{~h}$. After that, the reaction mixture was cooled at room temperature, followed by the addition of $1 \mathrm{~mL} \mathrm{MeOH}$ to quench the reaction. Mesitylene $(0.25$ mmol, $35 \mu \mathrm{L}$ ) was added as an internal standard, and yield was determined by GC-MS analysis. After that, the solvent was evaporated under reduced pressure, and the product (3a) was purified by column chromatography on silica gel using ethyl acetate/hexane as eluent. The product 3a was characterized by ${ }^{1} \mathrm{H}$ and ${ }^{13} \mathrm{C}$ NMR spectroscopy using $\mathrm{CDCl}_{3}$ as the solvent.

Table S5: Screening of catalyst.

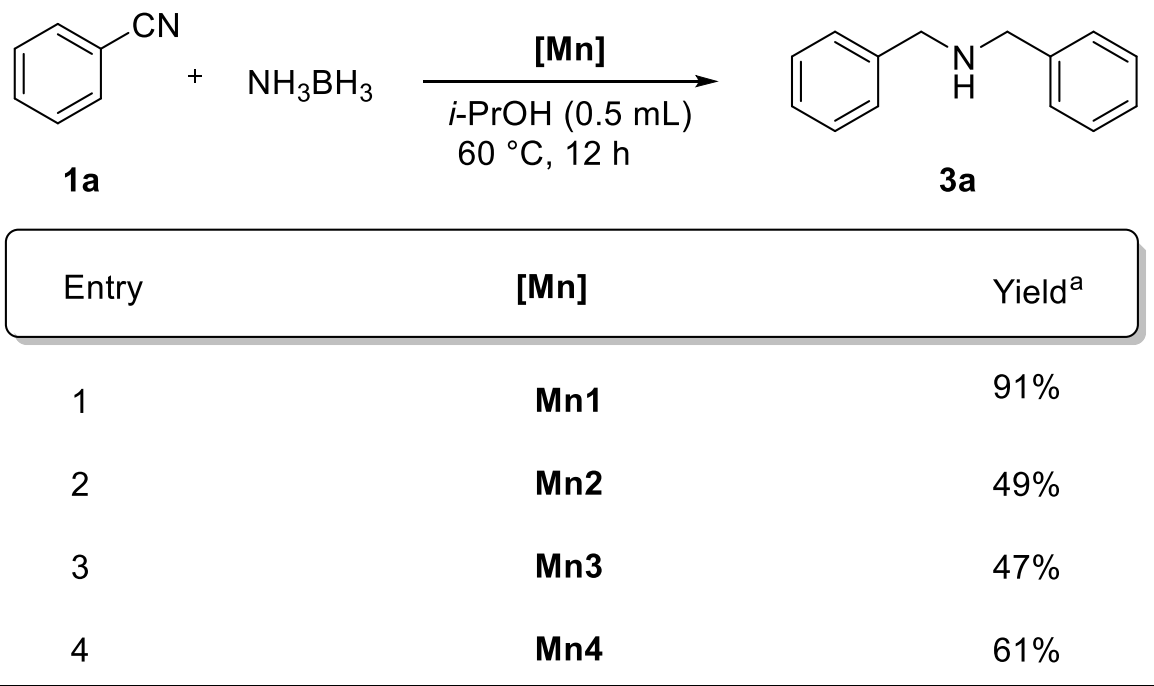

Reaction condition: $1 \mathrm{a}(0.25 \mathrm{mmol}), \mathrm{NH}_{3} \mathrm{BH}_{3}(0.75 \mathrm{mmol}),[\mathrm{Mn}](3 \mathrm{~mol} \%), i$-PrOH $(0.5 \mathrm{~mL}), 60^{\circ} \mathrm{C}$, Ar, $12 \mathrm{~h},{ }^{a}$ Yield was obtained by GC-MS analysis using mesitylene $(0.25 \mathrm{mmol})$ as an internal standard. 
Table S6: Screening of $\mathrm{NH}_{3} \mathrm{BH}_{3}$.

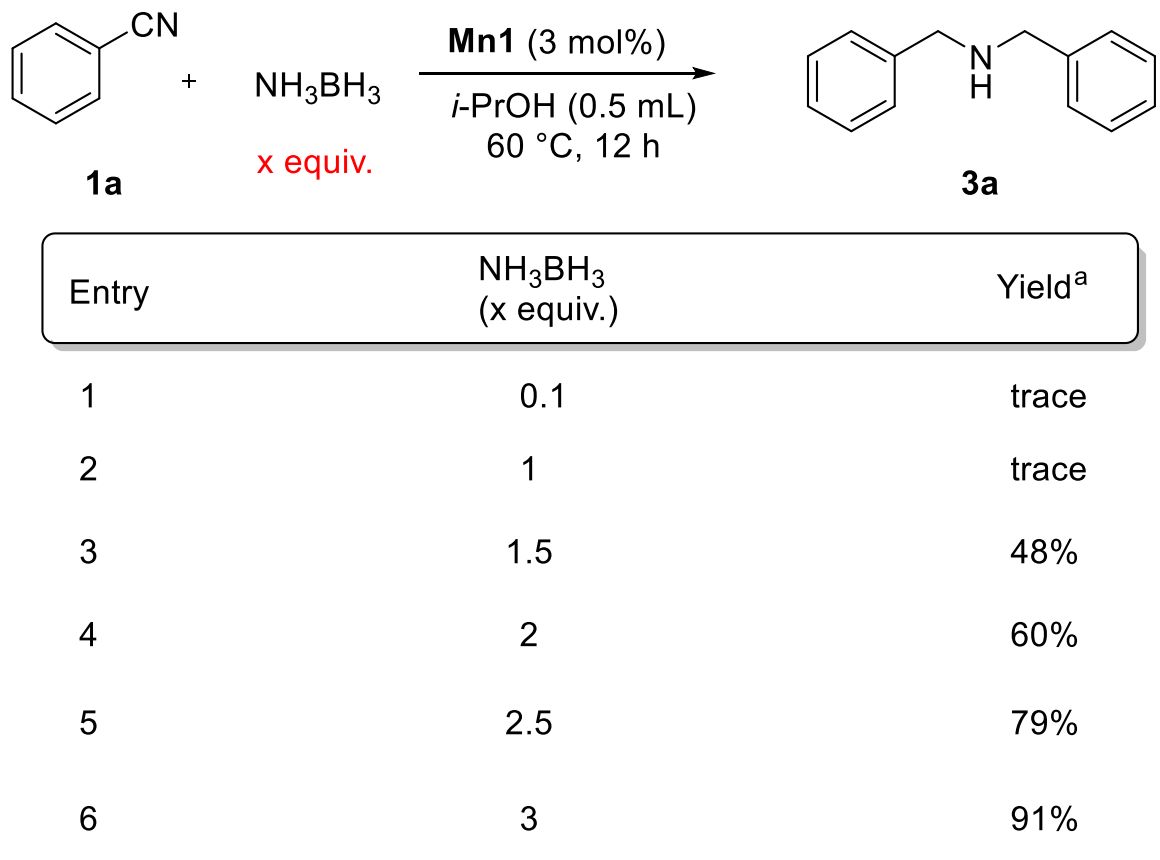

Reaction condition: 1a $(0.25 \mathrm{mmol}), \mathrm{NH}_{3} \mathrm{BH}_{3}$ (x equiv.), $\mathrm{Mn1}(3 \mathrm{~mol} \%), i-\mathrm{PrOH}(0.5 \mathrm{~mL}), 60{ }^{\circ} \mathrm{C}, \mathrm{Ar}$, $12 \mathrm{~h} .{ }^{\text {a }}$ Yield was obtained by GC-MS using mesitylene $(0.25 \mathrm{mmol})$ as an internal standard.

Table S7: Screening of Solvent.

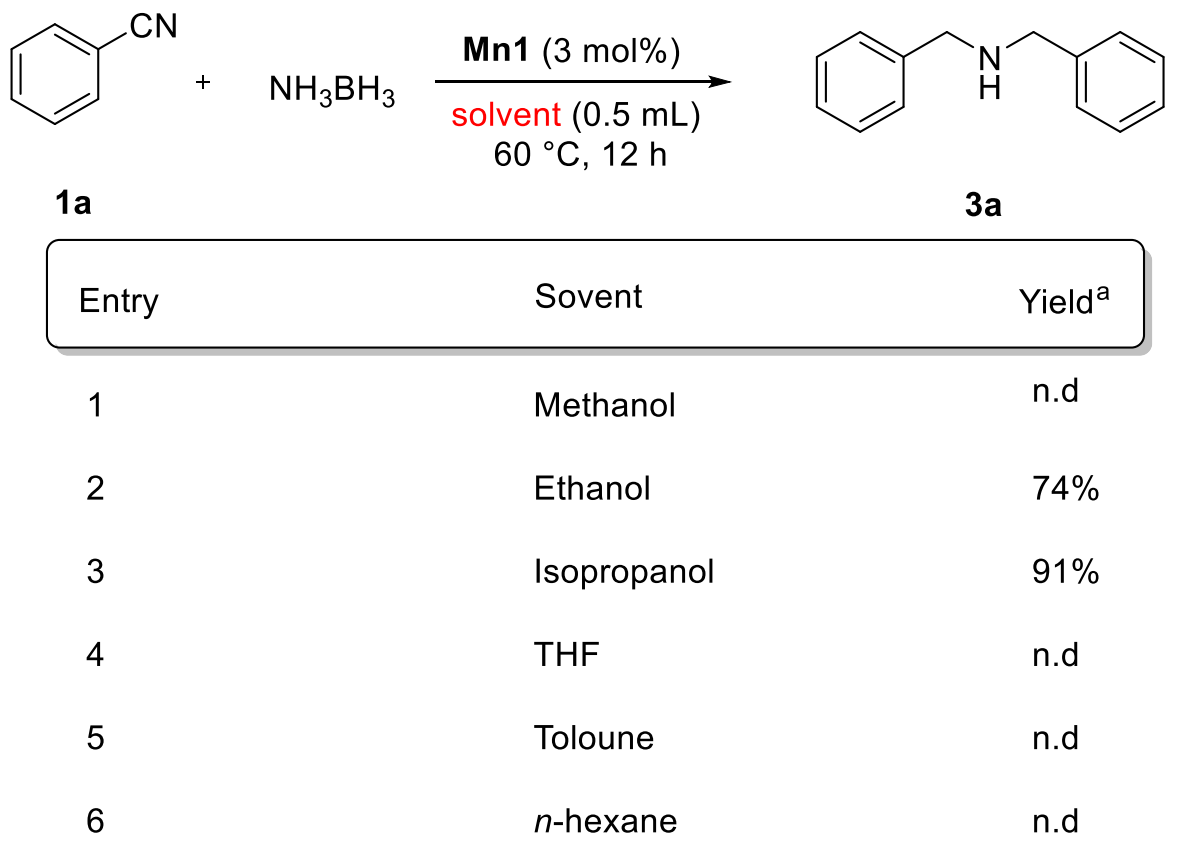

Reaction condition: $1 \mathrm{a}(0.25 \mathrm{mmol}), \mathrm{Mn} 1$ ( $3 \mathrm{~mol} \%), \mathrm{NH}_{3} \mathrm{BH}_{3}(0.75 \mathrm{mmol})$, solvent $(0.5 \mathrm{~mL}), 60^{\circ} \mathrm{C}$, $\mathrm{Ar}, 12 \mathrm{~h} .{ }^{\mathrm{a}}$ Yield was obtained by GC-MS analysis using mesitylene $(0.25 \mathrm{mmol})$ as an internal standard. 


\section{Detailed reaction optimization for asymmetrical secondary amine (4) synthesis}

\section{General procedure.}

In a $15 \mathrm{~mL}$ reaction tube, $\mathbf{M n} 2(3 \mathrm{~mol} \%, 3.5 \mathrm{mg})$ and $\mathrm{NH}_{3} \mathrm{BH}_{3}(0.75 \mathrm{mmol})$ were added under $\mathrm{Ar}$, followed by the addition of $i$-PrOH $(1 \mathrm{~mL})$, amine $(\mathbf{6 a})$, and nitrile (1a, $0.25 \mathrm{mmol}, 25.8 \mu \mathrm{L})$. The reaction tube was placed in a preheated oil bath at $80{ }^{\circ} \mathrm{C}$ for $24 \mathrm{~h}$. After that, the reaction mixture was cooled at room temperature, followed by the addition of $1 \mathrm{~mL} \mathrm{MeOH}$ to quench the reaction, and the solvent was evaporated under vacuum. Product $\mathbf{4}$ was purified by column chromatography on silica gel using ethyl acetate/hexane as eluent and characterized by ${ }^{1} \mathrm{H}$ and ${ }^{13} \mathrm{C}$ NMR spectroscopy using $\mathrm{CDCl}_{3}$ as the solvent.

Table S8: Reaction optimization of asymmetrical secondary amine.

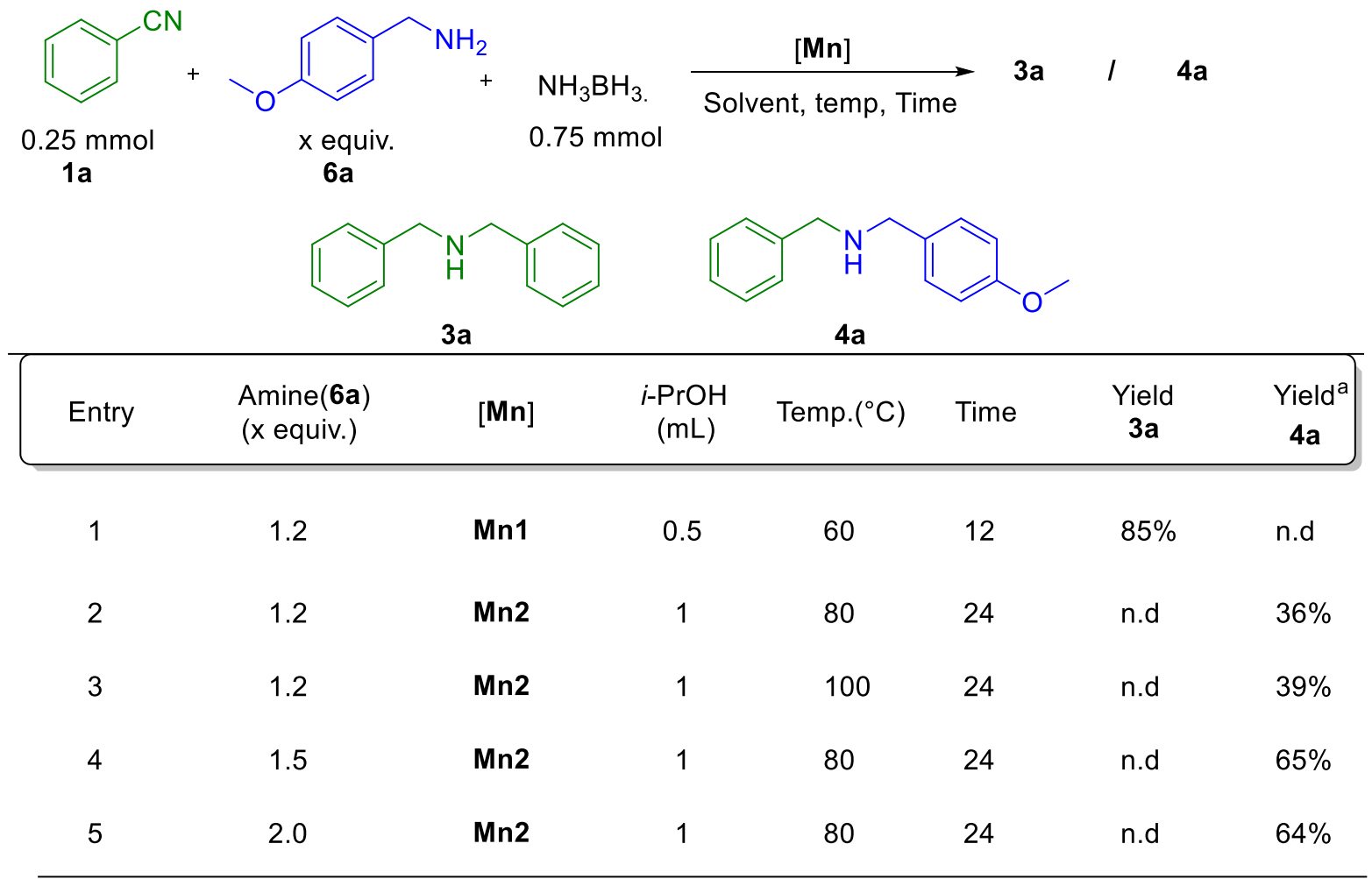

Reaction condition: $1 \mathrm{a}(0.25 \mathrm{mmol}), \mathbf{6 a}$ (x equiv.), $\mathrm{NH}_{3} \mathrm{BH}_{3}(0.75 \mathrm{mmol}),[\mathrm{Mn}](3 \mathrm{~mol} \%), i$-PrOH $(\mathrm{mL}), 80^{\circ} \mathrm{C}, \mathrm{Ar}, 24 \mathrm{~h} .{ }^{\mathrm{a}}$ Isolated yield. $\mathrm{n} . \mathrm{d}=$ not detected. 


\section{Mechanistic studies:}

\section{6a. IR analysis}

A solid-state ATR of Mn2 was taken. It shows a sharp peak at $2028 \mathrm{~cm}^{-1}$, and the peaks at 1940 and $1910 \mathrm{~cm}^{-1}$ were overlapped with each other.

In the second experiment, $\mathbf{M n} 2(0.05 \mathrm{mmol})$ was treated with $\mathrm{NH}_{3} \mathrm{BH}_{3}(0.15 \mathrm{mmol})$ in $2 \mathrm{~mL}$ THF solution at $60{ }^{\circ} \mathrm{C}$. The mixture was stirred for $2 \mathrm{~h}$. The solution was filtered through a syringe filter under Ar. The solvent was evaporated under vacuum, and solid-state ATR was measured for the remaining yellow solid.

In the third experiment, Mn2 $(0.05 \mathrm{mmol})$ catalyst was dissolved in THF $(2 \mathrm{~mL})$, and $t$-BuOK $(0.1$ mmol) was added under Ar. The color of the solution instantly changed from yellow to dark violet. The violet-colored solution was stirred for $2 \mathrm{~h}$ and filtered through a syringe filter under Ar. The solvent was evaporated under vacuum, and solid-state ATR was measured for the resulting solid.

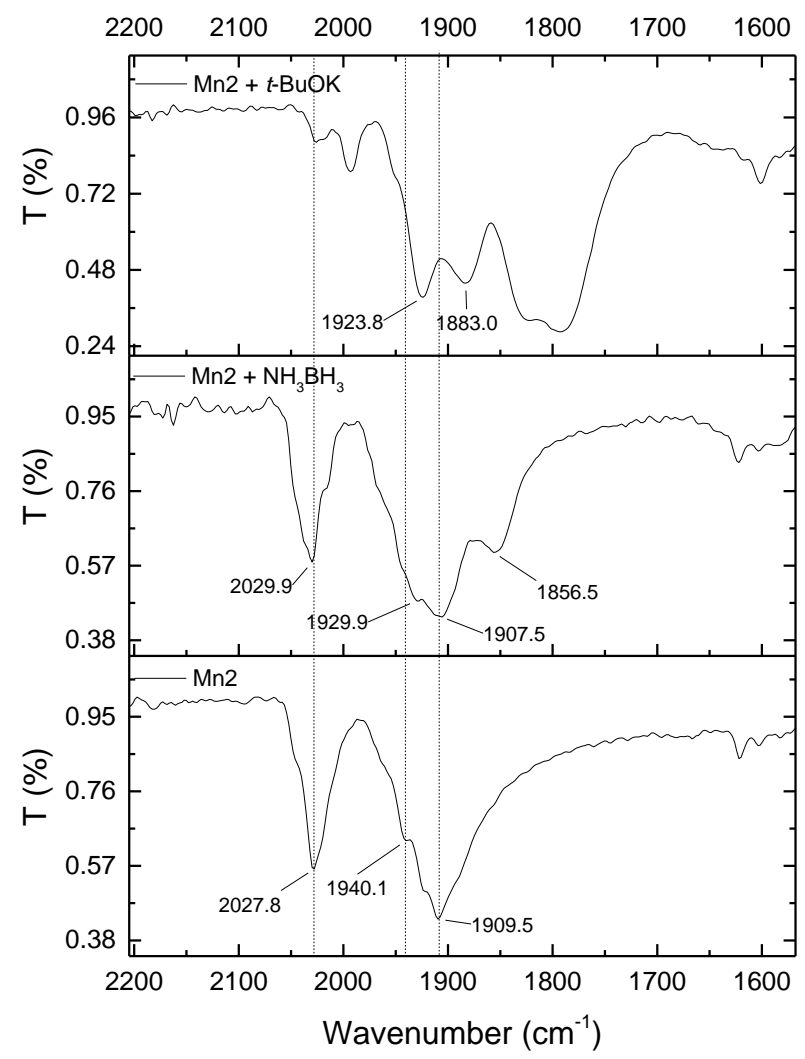

Figure S2. IR spectrum for a) Mn2 catalyst; b) Mn2 catalyst after treatment with ammonia borane; c) Mn2 after treatment with $t$-BuOK 
6b. ${ }^{11} \mathbf{B}$ NMR experiment at different time intervals: In an Ar filled glove box, $\mathrm{NH}_{3} \mathrm{BH}_{3}(0.45 \mathrm{mmol}$, $13.9 \mathrm{mg}), \mathrm{Mn} 2$ catalyst $(0.0045 \mathrm{mmol}, 2.1 \mathrm{mg})$ were taken in a J. Young NMR tube, and $0.5 \mathrm{~mL}$ of $\mathrm{C}_{6} \mathrm{D}_{6}$ was added to it followed by the addition of benzonitrile $(0.15 \mathrm{mmol}, 15.5 \mu \mathrm{L})$. The NMR tube was sealed, and ${ }^{11} \mathrm{~B}$ NMR of the resulting mixture was taken at different time intervals at room temperature.

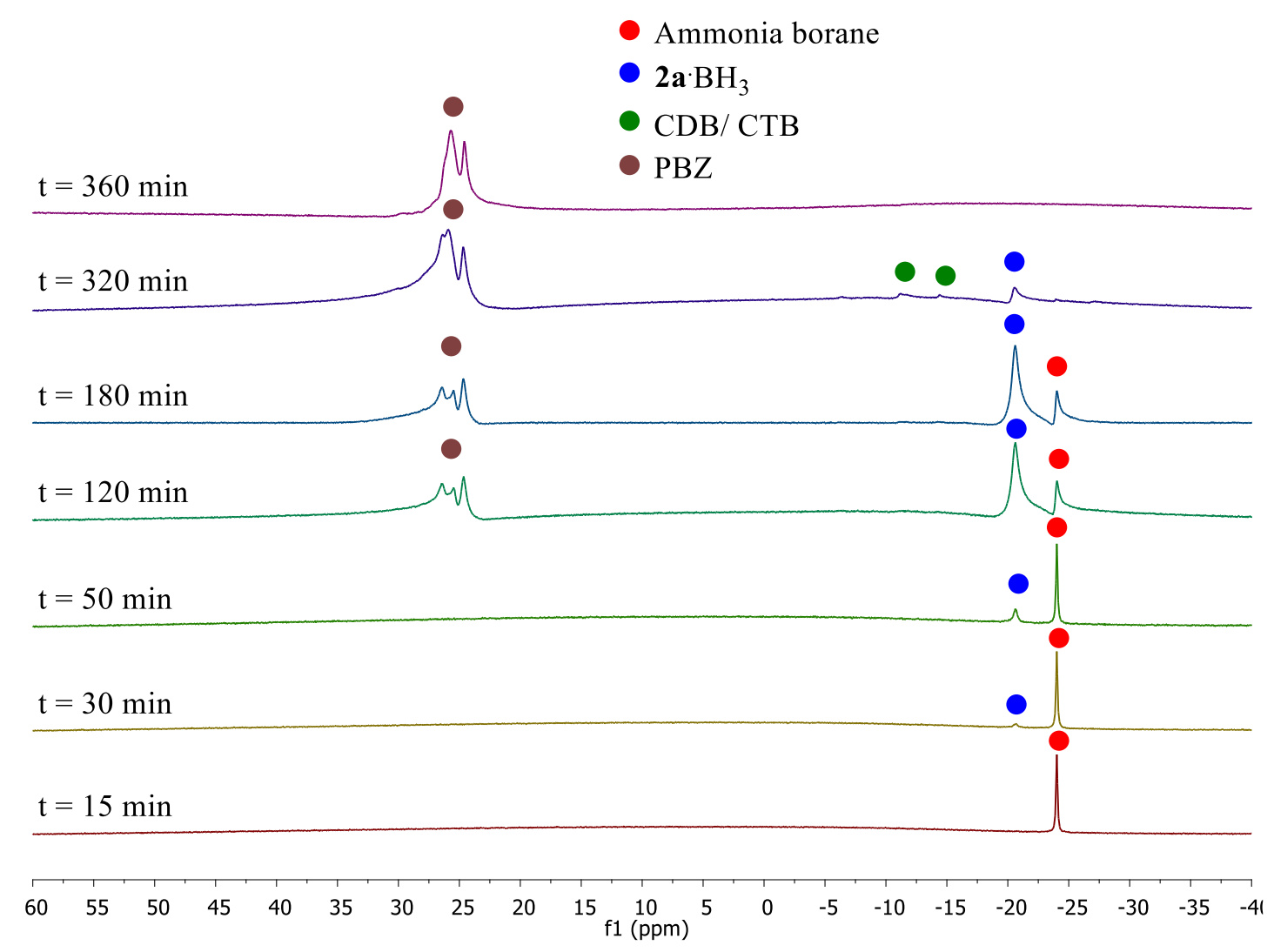

Figure S3. ${ }^{11} \mathrm{~B}$ NMR in $\mathrm{C}_{6} \mathrm{D}_{6}$. Where $\mathrm{CDB}=$ Cyclodiaminoborane, $\mathrm{CTB}=$ Cyclotriaminoborane, $\mathrm{PBZ}$ $=$ Polyborazine.

\section{6c. Isotope labeling experiment:}

$\mathrm{ND}_{3} \mathrm{BD}_{3}, \mathrm{NH}_{3} \mathrm{BD}_{3}$, and $\mathrm{ND}_{3} \mathrm{BH}_{3}$ were synthesized according to the literature procedure. ${ }^{4}$

Synthesis of $\mathrm{NH}_{3} \mathrm{BD}_{3} .{ }^{4 a} \mathrm{NaBD}_{4}(2.5 \mathrm{mmol})$ and powdered $\left(\mathrm{NH}_{4}\right)_{2} \mathrm{SO}_{4}(3.75 \mathrm{mmol})$ were added under Ar atmosphere to a $100 \mathrm{~mL}$ three-neck round bottom flask fitted with a stirrer, a stopper, and a condenser fitted with a connecting tube (one neck of the round-bottomed flask is connected with a dropping funnel, the middle neck is fitted with a condenser and the third neck was closed with a stopper) connecting tube was vented via an oil bubbler to the hood exhaust. THF $(20 \mathrm{~mL})$ was transferred into the flask via a dropping funnel, and the flask was stirred at $40{ }^{\circ} \mathrm{C}$ for $3 \mathrm{~h}$. After that, the reaction mixture was cooled to room temperature and filtered. The residue was washed with THF $(5 \mathrm{~mL})$. The filtrate was concentrated under reduced pressure to obtain $\mathrm{NH}_{3} \mathrm{BD}_{3}$ as a white solid. Yield $=66 \%$ (55 mg, 1.66 mmol).

Synthesis of $\mathbf{N D}_{3} \mathbf{B H}_{3 .}{ }^{4 b} \mathrm{NH}_{3} \mathrm{BH}_{3}(0.75 \mathrm{mmol})$ was taken in a Schlenk tube under Ar atmosphere, and then $1 \mathrm{~mL}$ of $\mathrm{D}_{2} \mathrm{O}$ was added to it. The mixture was stirred until $\mathrm{NH}_{3} \mathrm{BH}_{3}$ completely dissolves in $\mathrm{D}_{2} \mathrm{O}$ (typically 5 mins) to furnish a clear, colorless solution. The solvent was then vacuum distilled, and the white crystalline residue was dried under vacuum. This process was repeated three times to get the $\mathrm{ND}_{3} \mathrm{BH}_{3}$ with $99 \%$ incorporation of deuterium.

Synthesis of $\mathbf{N D}_{3} \mathbf{B D}_{3 .}{ }^{4 \mathrm{~b}} \mathrm{NH}_{3} \mathrm{BD}_{3}(0.75 \mathrm{mmol})$ was taken in a Schlenk tube under Ar atmosphere, and then $1 \mathrm{~mL}$ of $\mathrm{D}_{2} \mathrm{O}$ was added to it. The mixture was stirred until $\mathrm{NH}_{3} \mathrm{BD}_{3}$ completely dissolves in $\mathrm{D}_{2} \mathrm{O}$ 
(typically 5 mins) to furnish a clear, colorless solution. The solvent was then vacuum distilled, and the white crystalline residue was dried under vacuum. This process was repeated three times to get the $\mathrm{ND}_{3} \mathrm{BD}_{3}$ with $99 \%$ incorporation of deuterium.

\section{General procedure for the synthesis of $\mathbf{d}_{2}$-benzylamine:}

Following the standard condition, $\mathbf{M n} 2(3 \mathrm{~mol} \%, 1.4 \mathrm{mg})$ and $\mathrm{NH}_{3} \mathrm{BD}_{3}(0.3 \mathrm{mmol}, 10 \mathrm{mg})$ were dissolved in THF $(0.4 \mathrm{~mL})$ in the reaction tube under Ar, followed by the addition of benzonitrile $(0.1$ mmol, $10.4 \mu \mathrm{L}$ ). The reaction mixture was stirred at $60{ }^{\circ} \mathrm{C}$ for $6 \mathrm{~h}$. After that, the reaction was cooled to room temperature and then filtered through celite bed. The filtrate was dried under the vacuum. The amine product was characterized by ${ }^{1} \mathrm{H} \mathrm{NMR}$ in $\mathrm{CDCl}_{3}$ solvent.

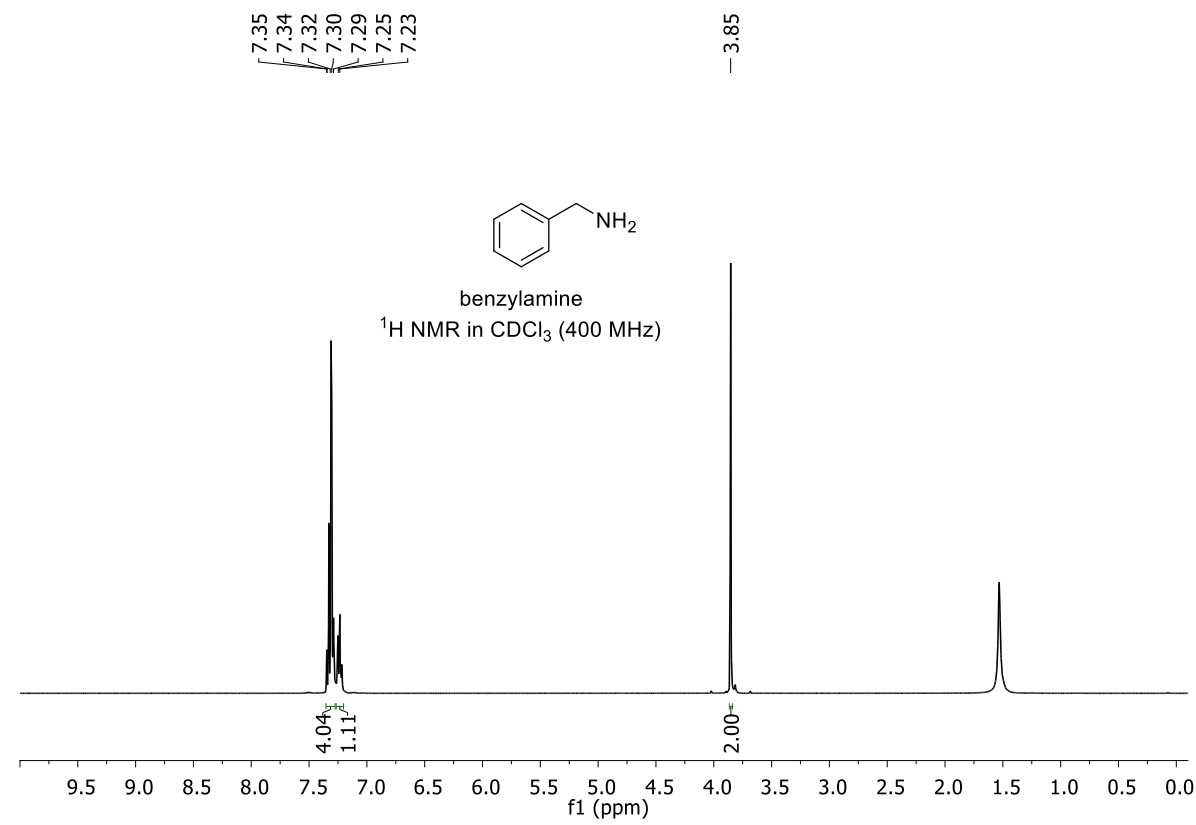

Figure S4. ${ }^{1} \mathbf{H}$ NMR spectrum of $\mathbf{2 a}$ in $\mathrm{CDCl}_{3}$

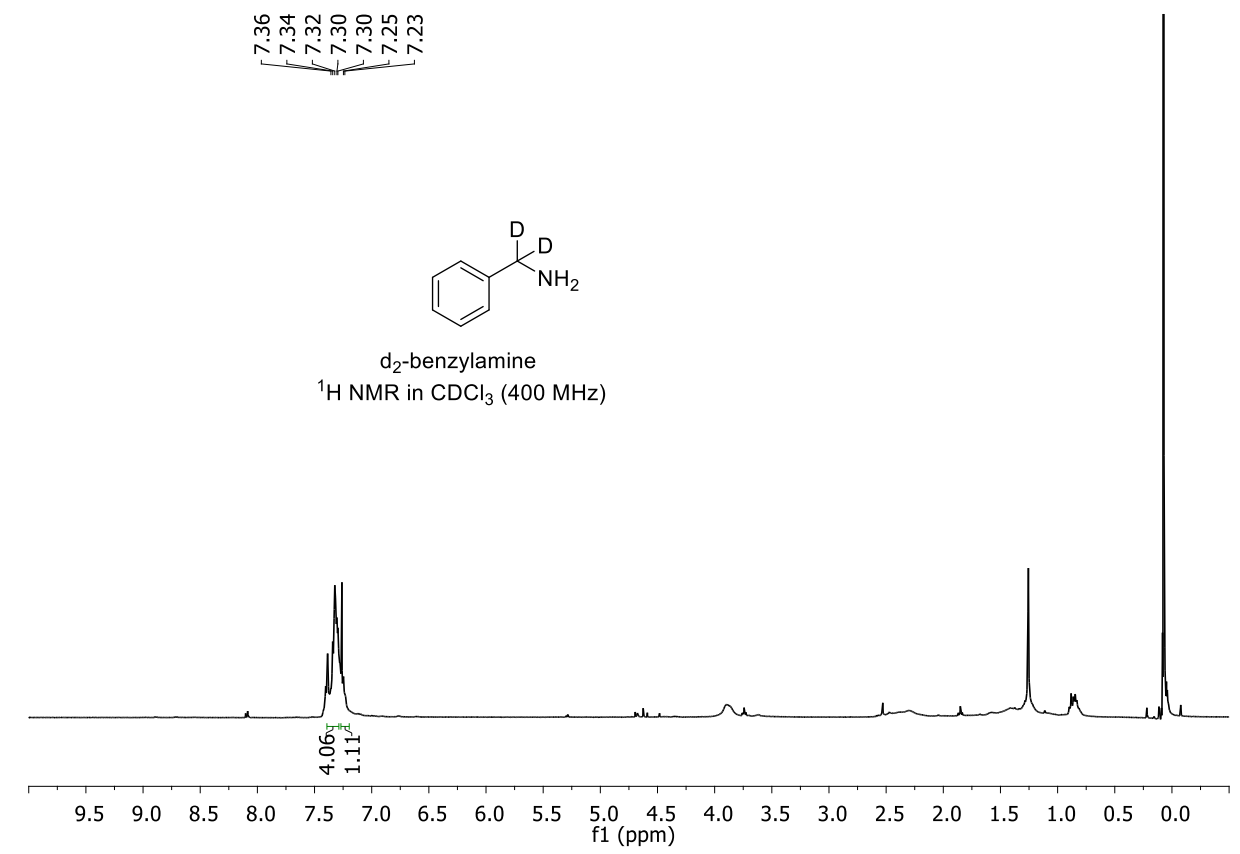

Figure S5. ${ }^{1} \mathrm{H}$ NMR spectrum of $\mathrm{d}_{2}-\mathbf{2 a}$ in $\mathrm{CDCl}_{3}$. 


\section{6d. Control experiment: Probing the hemilability by adding external ligands:}

In an oven-dried reaction tube, $\mathrm{NH}_{3} \mathrm{BH}_{3}(0.3 \mathrm{mmol}, 9.3 \mathrm{mg}), \mathbf{M n} 2(3 \mathrm{~mol} \%, 1.3 \mathrm{mg})$, and additive (20 mol\%) were added under Ar. To this, $n$-hexane $(0.1 \mathrm{~mL})$ was added, followed by the addition of benzonitrile $(0.1 \mathrm{mmol}, 10.3 \mu \mathrm{L})$. The reaction tube was sealed and placed in a preheated oil bath at 60 ${ }^{\circ} \mathrm{C}$ and stirred for $6 \mathrm{~h}$. Then, the reaction mixture was quenched by the addition of methanol $(1 \mathrm{~mL})$. Mesitylene ( $0.1 \mathrm{mmol}, 14 \mu \mathrm{L}$, internal standard) was added, and the yield was determined by the GCMS analysis.

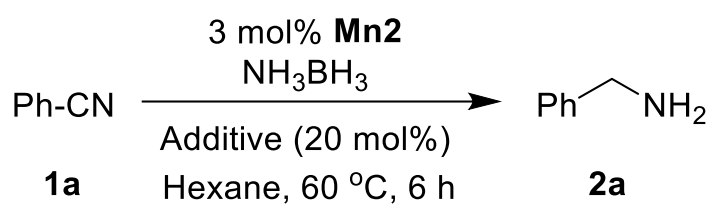

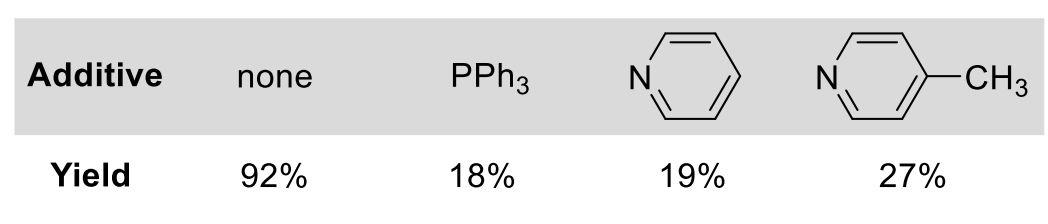

Table S9: Controlled experiments in the presence of additives. 


\section{Kinetic experiments:}

The rate of the reaction was determined according to the initial rate law method (up to $45 \%$ yield of 2a). The kinetic experiment was done using GC-MS by collecting multiple data points (3-12) at the initial stage of the reaction up to 30 to $35 \mathrm{~min}$. The reaction rate was determined by the least-square fit method with the formation of the initial concentration of product $2 \mathbf{a}(\mathrm{M})$ versus time.

General method: In an oven-dried Schlenk tube, $\mathrm{NH}_{3} \mathrm{BH}_{3}$ and $\mathbf{M n} 2$ were added under Ar. To this, toluene or tetrahydrofuran was added, followed by $n$-decane (internal standard) and benzonitrile (1a). The Schlenk tube was sealed with a septum and placed in a preheated oil bath at $60{ }^{\circ} \mathrm{C}$ maintaining the Ar atmosphere. At a constant interval of either 1 or $2 \mathrm{~min}$, the aliquot was collected, and the yield of $\mathbf{2 a}$ (M) was monitored by GC-MS.

\section{7a. Determination of reaction order for $\mathrm{Mn} 2$ in toluene:}

The concentration of $\mathbf{M n 2}$ was varied while keeping a constant concentration of $\mathrm{NH}_{3} \mathrm{BH}_{3}$ and $\mathbf{1 a}$ in toluene.

\begin{tabular}{|c|c|c|c|c|c|}
\hline Entry & $\mathbf{N H}_{\mathbf{3}} \mathbf{B H}_{\mathbf{3}} \mathbf{( M )}$ & $\mathbf{1 a}(\mathbf{M})$ & $\mathbf{M n 2} \mathbf{( M )}$ & Initial rate(r) (M/min) & $\mathbf{R}^{\mathbf{2}}$ \\
\hline $\mathrm{A}$ & 0.75 & 0.25 & 0.0075 & 0.0048 & 0.962 \\
\hline $\mathrm{B}$ & 0.75 & 0.25 & 0.0150 & 0.0088 & 0.953 \\
\hline $\mathrm{C}$ & 0.75 & 0.25 & 0.0225 & 0.0112 & 0.972 \\
\hline $\mathrm{D}$ & 0.75 & 0.25 & 0.0300 & 0.0160 & 0.999 \\
\hline
\end{tabular}

a)

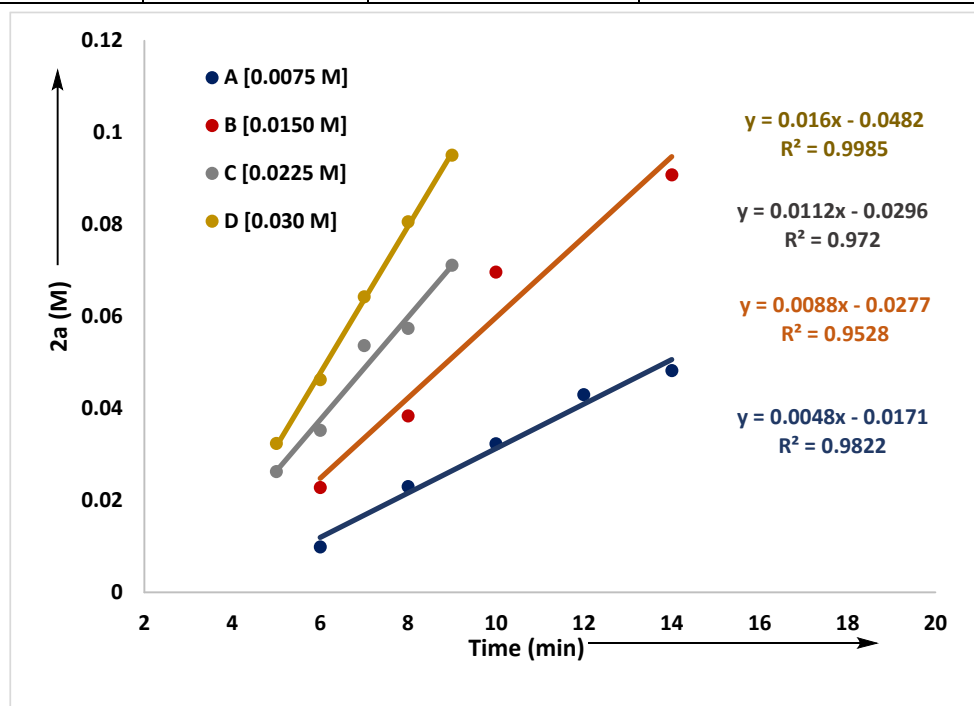

b)

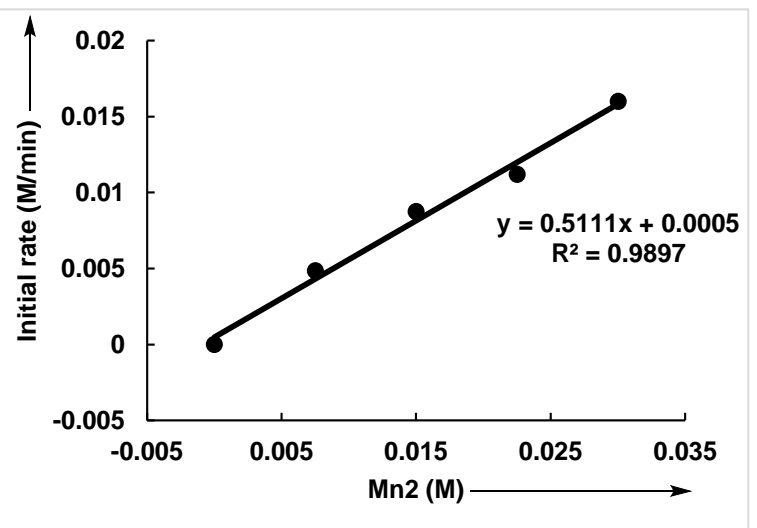

Figure S6. (a) Plot of [2a] vs time for varying concentrations of Mn2, A [0.0075 M], B [0.0150 M], C [0.0225 M], D [0.0300 M]; (b) Plot of Initial rate vs [Mn2]. 
7b. Determination of reaction order for $1 \mathrm{a}$ in toluene:

The concentration of $\mathbf{1 a}$ was varied while keeping a constant concentration of $\mathbf{M n} \mathbf{2}$ and $\mathrm{NH}_{3} \mathrm{BH}_{3}$.

\begin{tabular}{|c|c|c|c|c|c|}
\hline Entry & $\mathbf{N H}_{\mathbf{3}} \mathbf{B H}_{\mathbf{3}} \mathbf{( M )}$ & $\mathbf{1 a}(\mathbf{M})$ & $\mathbf{M n 2} \mathbf{( M )}$ & Initial rate(r) (M/min) & $\mathbf{R}^{2}$ \\
\hline $\mathrm{A}^{\prime}$ & 0.75 & 0.250 & 0.0075 & 0.0048 & 0.982 \\
\hline B $^{\prime}$ & 0.75 & 0.375 & 0.0075 & 0.0070 & 0.991 \\
\hline C' $^{\prime}$ & 0.75 & 0.500 & 0.0075 & 0.0058 & 0.994 \\
\hline D' $^{\prime}$ & 0.75 & 0.625 & 0.0075 & 0.0068 & 0.977 \\
\hline
\end{tabular}

a)

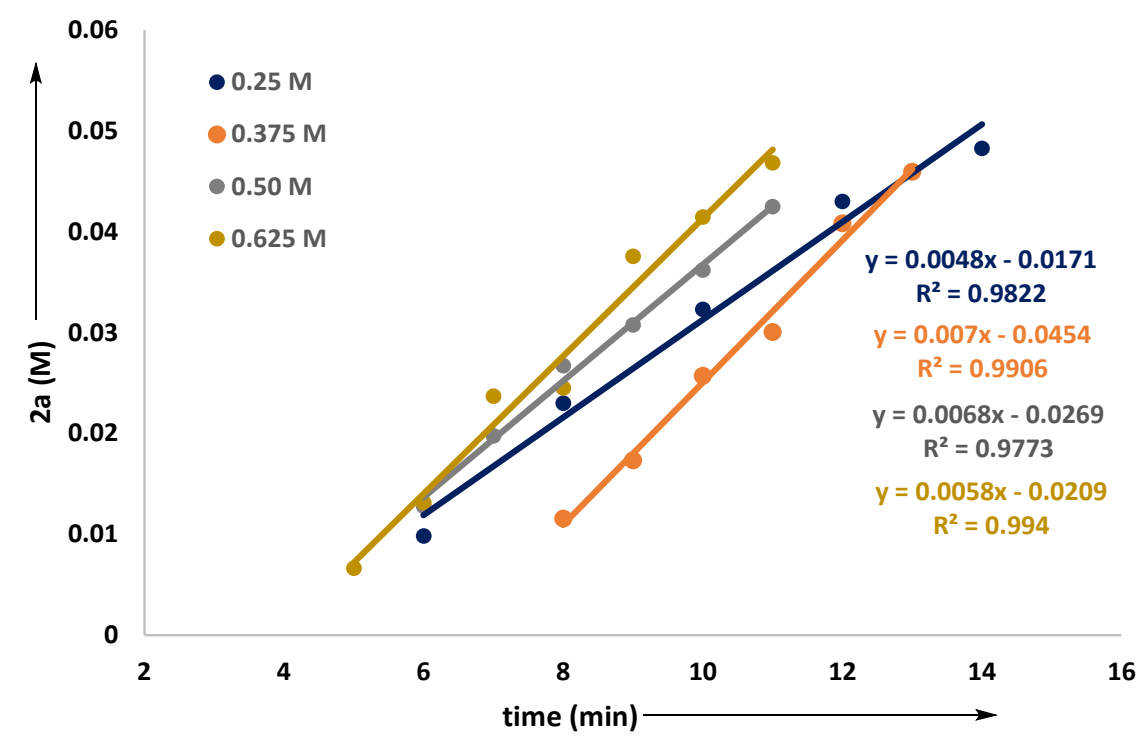

b)

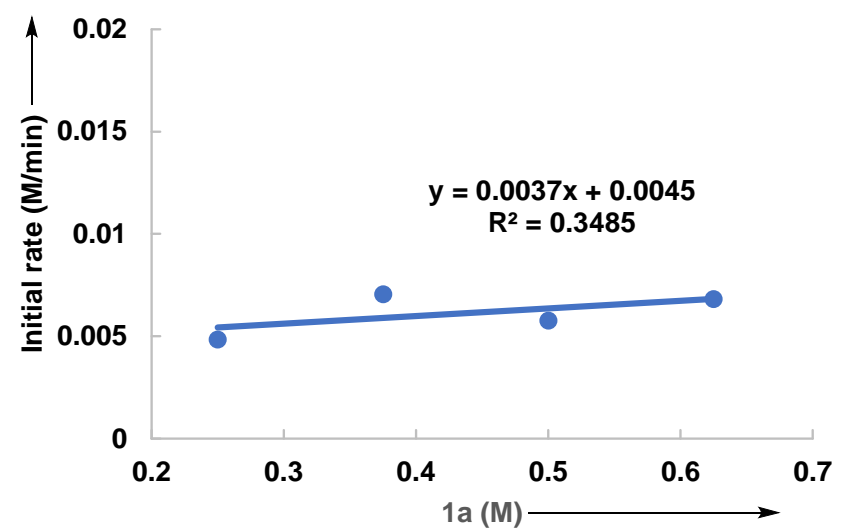

Figure S7. (a) Plot of [2a] vs time for varying concentrations of 1a, A [0.25 M], B [0.375 M], C [0.500 M], D [0.625 M]; (b) Plot of Initial rate vs [1a]. 
7c. Determination of reaction order for $\mathrm{NH}_{3} \mathrm{BH}_{3}$ in toluene:

The concentration of $\mathrm{NH}_{3} \mathrm{BH}_{3}$ was varied while keeping a constant concentration of $\mathbf{M n} 2$ and $\mathbf{1 a}$ in toluene.

\begin{tabular}{|c|c|c|c|c|c|}
\hline Entry & $\mathbf{N H}_{3} \mathbf{B H}_{3}(\mathbf{M})$ & $\mathbf{1 a}(\mathbf{M})$ & $\mathbf{M n 2}(\mathbf{M})$ & Initial rate(r) (M/min) & $\mathbf{R}^{2}$ \\
\hline A" & 0.25 & 0.25 & 0.0075 & 0.0062 & 0.992 \\
\hline B" & 0.75 & 0.25 & 0.0075 & 0.0048 & 0.982 \\
\hline C" & 1.00 & 0.25 & 0.0075 & 0.0060 & 0.998 \\
\hline D" & 1.25 & 0.25 & 0.0075 & 0.0048 & 0.974 \\
\hline
\end{tabular}

a)

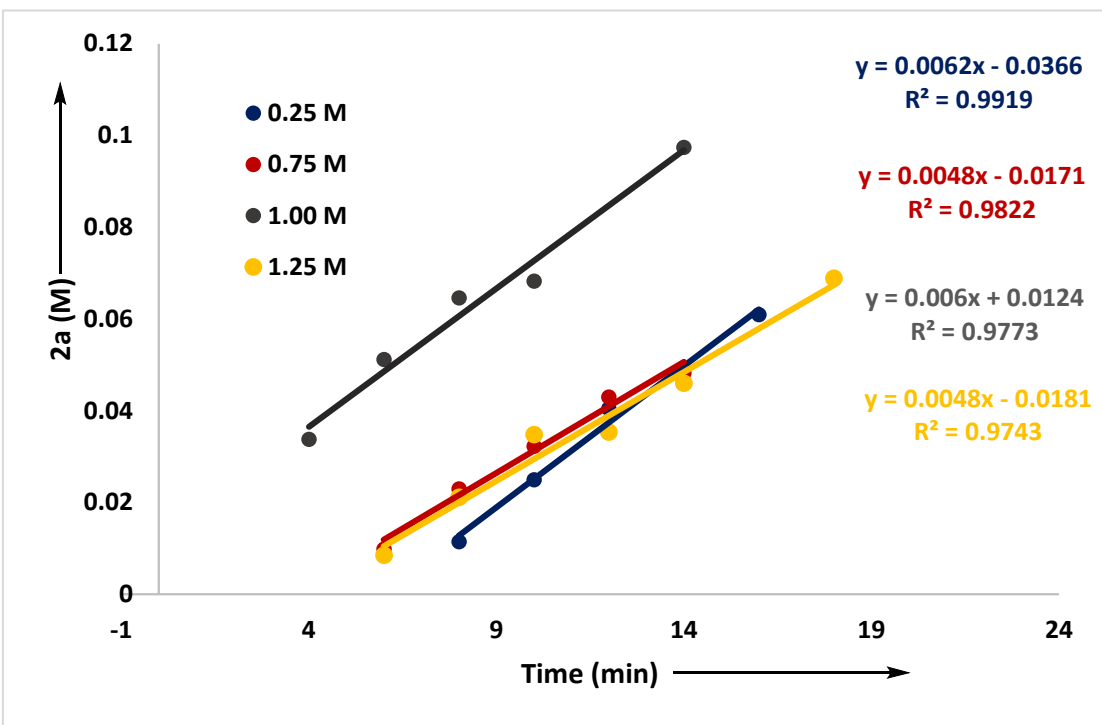

b)

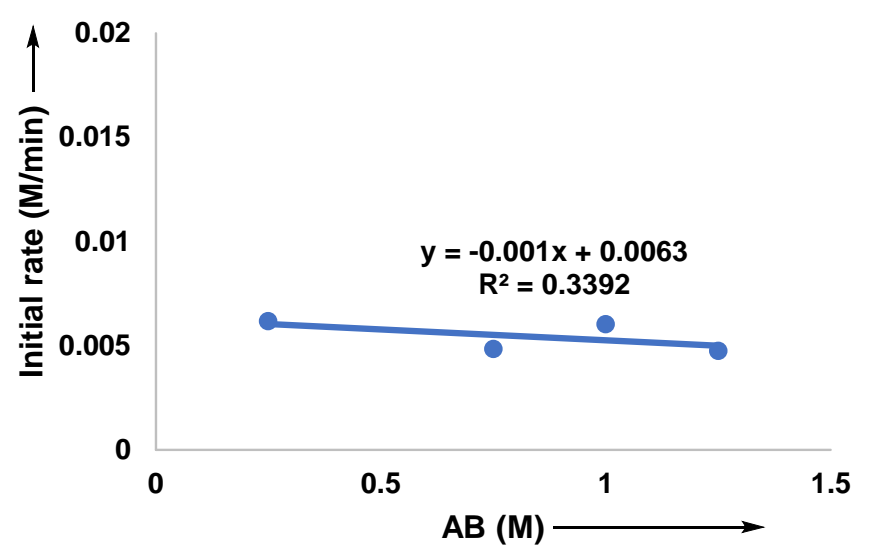

Figure S8. (a) Plot of [2a] vs time for varying concentrations of AB. A $[0.25 \mathrm{M}], \mathbf{B}[0.75 \mathrm{M}], \mathbf{C}[1.00$ $\mathrm{M}], \mathbf{D}[1.25 \mathrm{M}]$; (b) Plot of Initial rate vs [AB]. 
7d. Determination of reaction order for Mn2 in THF:

The concentration of $\mathbf{M n} 2$ was varied while keeping constant the concentration of $\mathrm{NH}_{3} \mathrm{BH}_{3}$ and $\mathbf{1 a}$ in THF

\begin{tabular}{|c|c|c|c|c|c|}
\hline Entry & 1a[M] & $\mathbf{A B}[\mathbf{M}]$ & $\mathbf{M n 2}[\mathbf{M}]$ & Initial rate (r) [M/min] & $\mathbf{R}^{\mathbf{2}}$ \\
\hline A $^{\prime}$ & 0.25 & 0.75 & 0.0075 & 0.0107 & 0.989 \\
\hline B' $^{\prime}$ & 0.25 & 0.75 & 0.0150 & 0.0146 & 0.995 \\
\hline C' $^{\prime}$ & 0.25 & 0.75 & 0.0225 & 0.0231 & 0.993 \\
\hline
\end{tabular}

a)

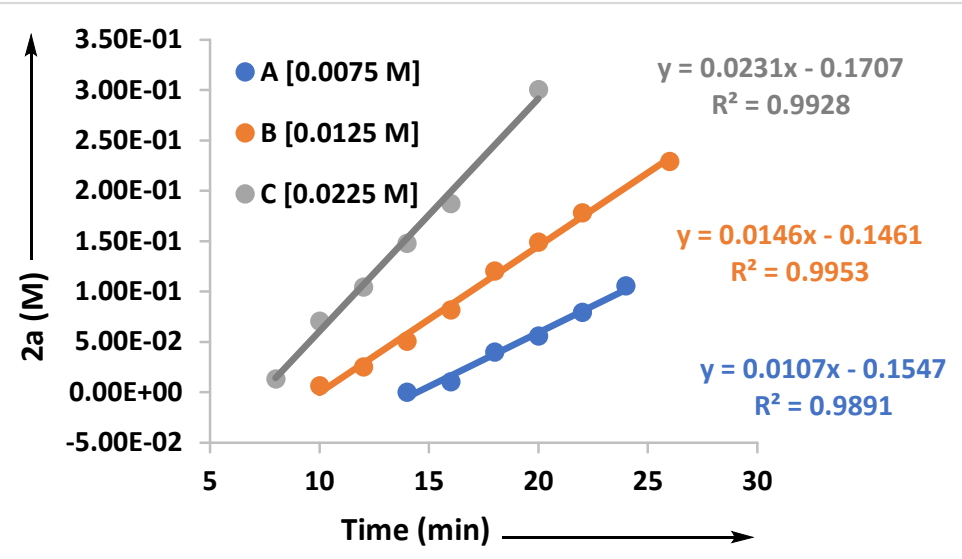

b)

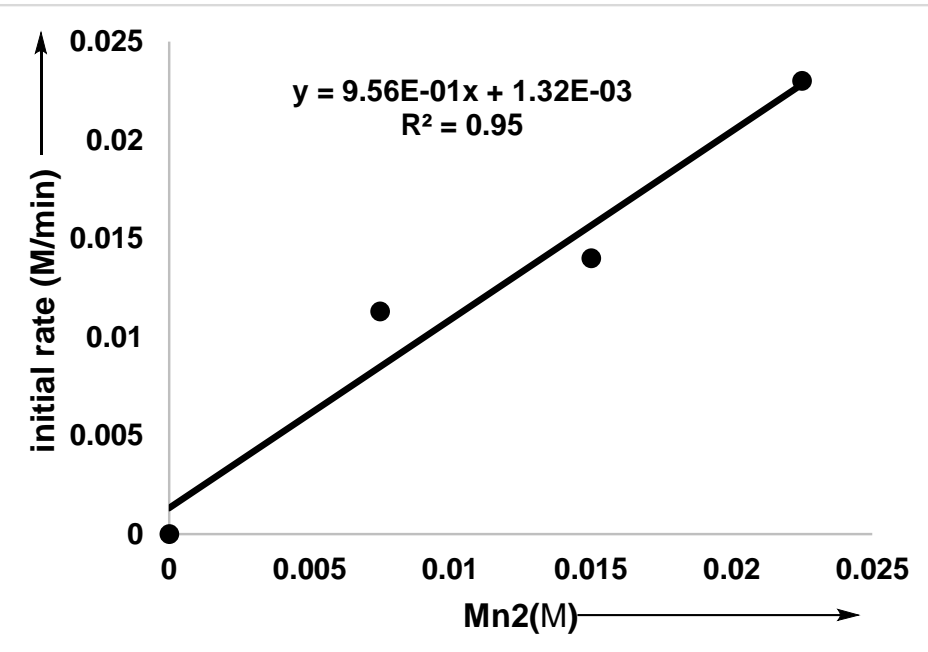

Figure S9. (a) Plot of [2a] vs time for varying concentrations of Mn2, A [0.0075 M], B [0.0150 M], C [0.0225 M]; (b) Plot of Initial rate vs [Mn2]. 
7e. Determination of reaction order for $\mathrm{NH}_{3} \mathrm{BH}_{3}$ in THF:

The concentration of $\mathrm{NH}_{3} \mathrm{BH}_{3}$ was varied while keeping the constant concentration of $\mathbf{M n} \mathbf{2}$ and $\mathbf{1 a}$ in THF.

\begin{tabular}{|c|c|c|c|c|c|}
\hline Entry & 1a [M] & AB [M] & Mn2 [M] & Initial rate(r) [M/min] & $\mathbf{R}^{\mathbf{2}}$ \\
\hline A $^{\prime}$ & 0.25 & 0.25 & 0.0075 & 0.0027 & 0.990 \\
\hline B' & 0.25 & 0.50 & 0.0075 & 0.0051 & 0.989 \\
\hline C' $^{\prime}$ & 0.25 & 0.75 & 0.0075 & 0.0107 & 0.989 \\
\hline D' & 0.25 & 1.00 & 0.0075 & 0.0137 & 0.924 \\
\hline
\end{tabular}

a)

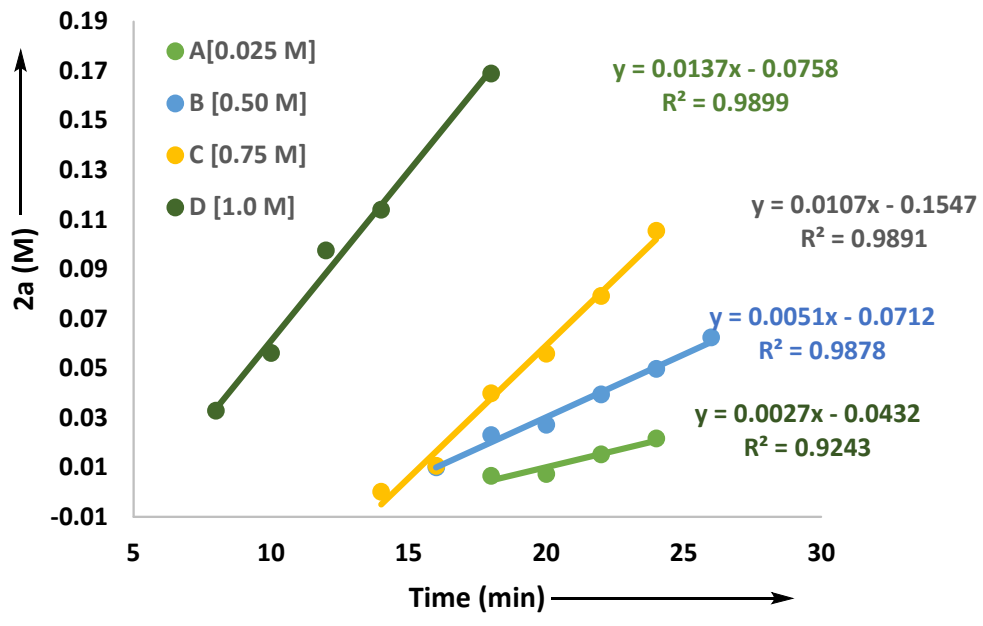

b)

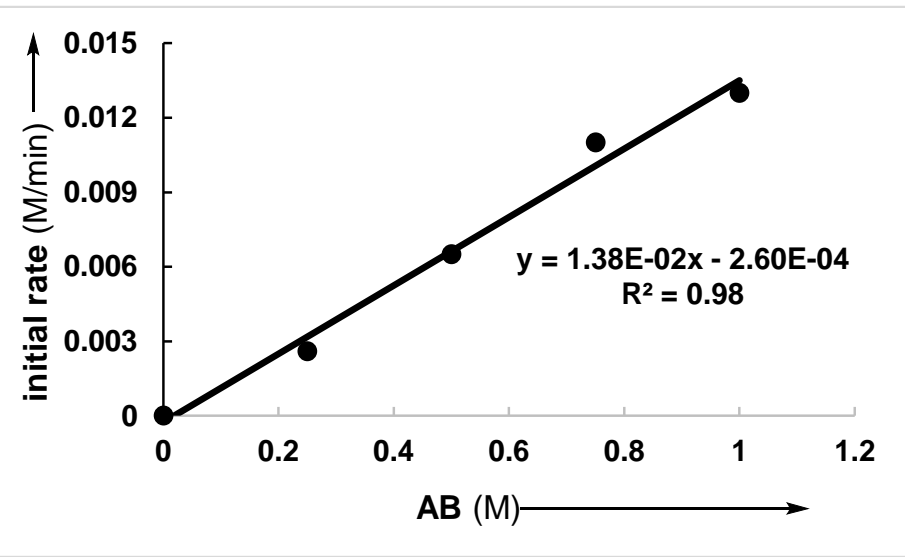

Figure S10. (a) Plot of [2a] vs time for varying concentrations of $\mathrm{NH}_{3} \mathrm{BH}_{3}, \mathbf{A}[0.25 \mathrm{M}], \mathbf{B}[0.50 \mathrm{M}], \mathbf{C}$ [0.75 M], D [1.0 M]; (b) Plot of Initial rate vs [AB]. 
7f. Determination of reaction order for $1 \mathrm{a}$ in $\mathrm{THF}$.

The concentration of $\mathbf{1 a}$ was varied while keeping the constant concentration of $\mathbf{M n} \mathbf{2}$ and $\mathrm{NH}_{3} \mathrm{BH}_{3}$ in THF.

\begin{tabular}{|c|c|c|c|c|c|}
\hline Entry & 1a (M) & AB (M) & Mn2 (M) & Initial rate(r)(M/min) & $\mathbf{R}^{\mathbf{2}}$ \\
\hline A & 0.25 & 0.75 & 0.0075 & 0.0107 & 0.989 \\
\hline B & 0.50 & 0.75 & 0.0075 & 0.0126 & 0.960 \\
\hline C' & 0.75 & 0.75 & 0.0075 & 0.0123 & 0.951 \\
\hline D" & 1.0 & 0.75 & 0.0075 & 0.0159 & 0.993 \\
\hline
\end{tabular}

a)

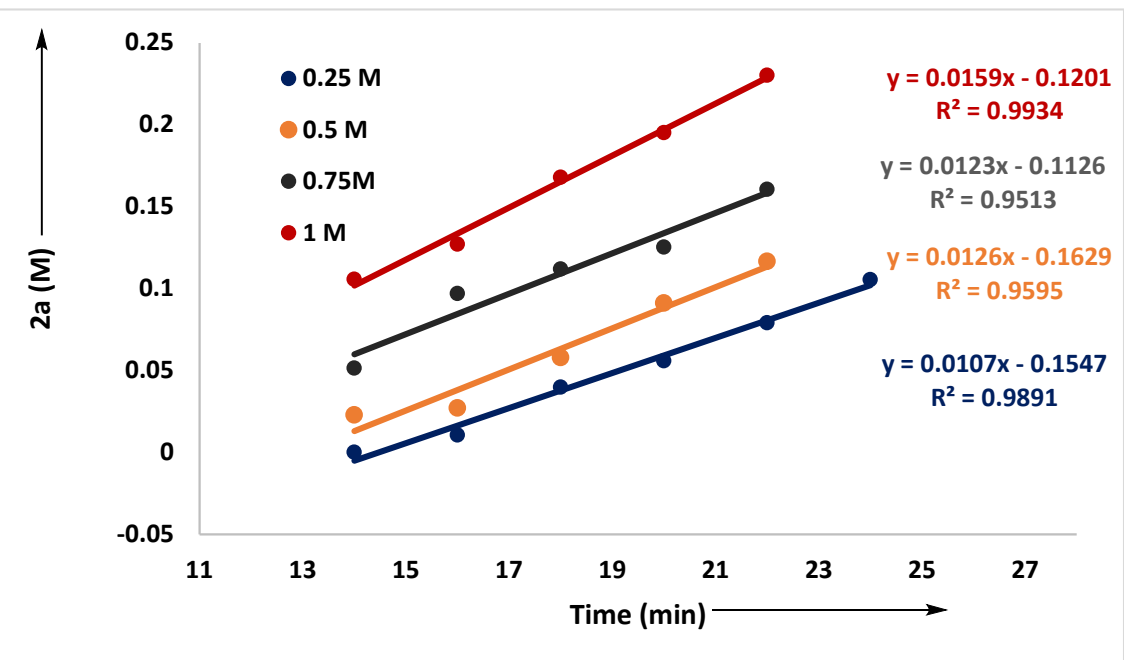

b)

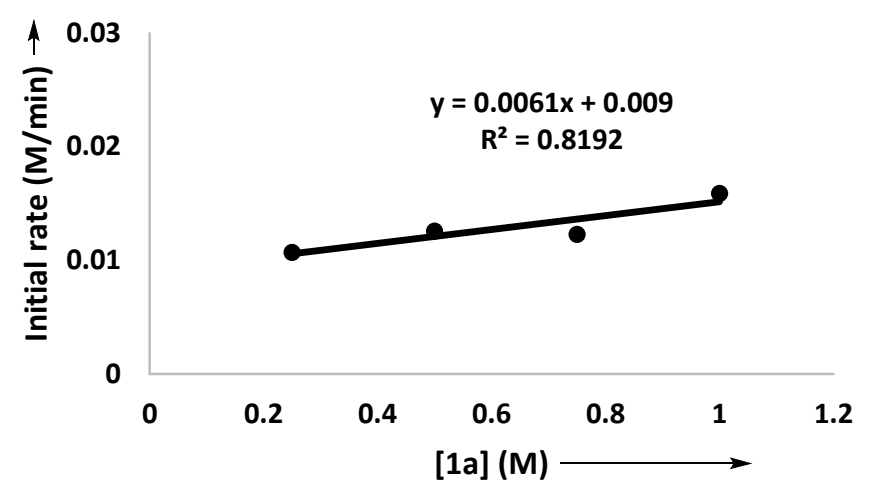

Figure S11. (a) Plot of [2a] vs time for varying concentrations of $\mathrm{NH}_{3} \mathrm{BH}_{3}, \mathbf{A}[0.25 \mathrm{M}], \mathbf{B}[0.50 \mathrm{M}], \mathbf{C}$ [0.75 M], D [1.0 M]; (b) Plot of Initial rate vs [1a]. 


\section{7g. Eyring analysis:}

In an oven-dried Schlenk tube, $\mathrm{NH}_{3} \mathrm{BH}_{3}(0.9 \mathrm{mmol}, 27.8 \mathrm{mg})$ and $\mathbf{M n} 2(3 \mathrm{~mol} \%, 4.2 \mathrm{mg})$ were taken under Ar. To this, THF $(1.2 \mathrm{~mL})$ was added, and the resulting solution was stirred for 2 min to get a homogeneous mixture. Then $n$-decane $(0.3 \mathrm{mmol}, 58.5 \mu \mathrm{L})$ and $1 \mathrm{a}(0.3 \mathrm{mmol}, 31 \mu \mathrm{L})$ were added at room temperature and quickly transferred to a preheated oil bath set at different temperatures $45^{\circ} \mathrm{C}, 50$ ${ }^{\circ} \mathrm{C}, 55^{\circ} \mathrm{C}$, and $60{ }^{\circ} \mathrm{C}$. The aliquot was collected at $2 \mathrm{~min}$ interval of time, and the yield was monitored by GC-MS.

\begin{tabular}{|c|c|c|c|c|}
\hline $\boldsymbol{k}[\mathbf{M} / \mathbf{m i n}]$ & $\mathbf{T}(\mathbf{K})$ & $\mathbf{1} / \mathbf{T}$ & $\ln (\boldsymbol{k} / \mathbf{T})$ & $\ln (\boldsymbol{k})$ \\
\hline $3.56 \mathrm{e}-03$ & 318 & 0.003145 & -11.40 & -5.63799 \\
\hline $5.49 \mathrm{e}-03$ & 323 & 0.003096 & -10.9825 & -5.20483 \\
\hline $8.19 \mathrm{e}-03$ & 328 & 0.003049 & -10.5979 & -4.80484 \\
\hline $1.10 \mathrm{e}-02$ & 333 & 0.003003 & -10.318 & -4.50986 \\
\hline
\end{tabular}

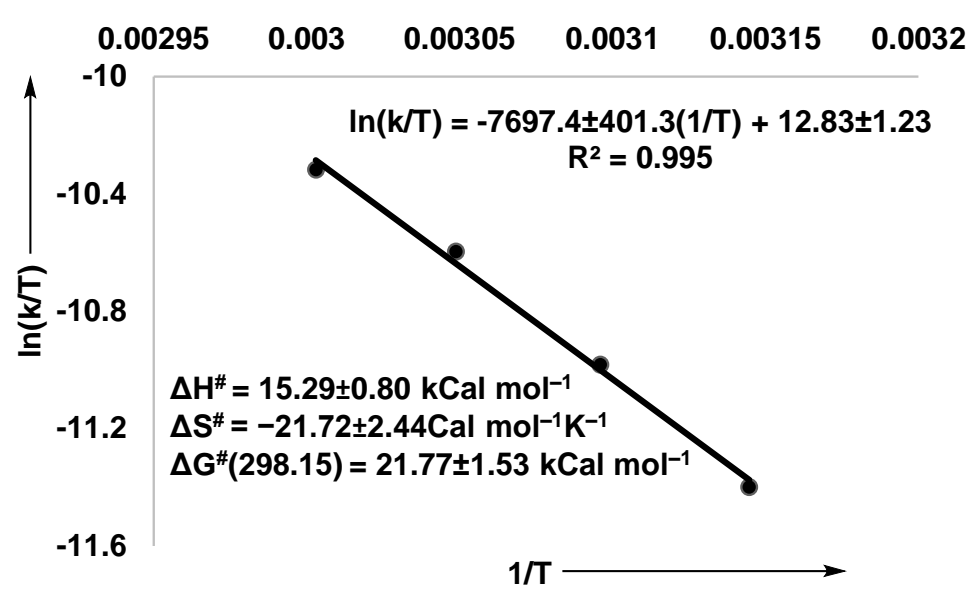

Figure S12. Eyring plot for the Mn2 catalyst transfer hydrogenation of 1a with AB in THF. Based on respective equations, activation thermodynamic parameters were calculated

\section{7h. Determination of Kinetic Isotope effect (KIE):}

In an oven-dried reaction tube $\mathrm{Mn} 2(3 \mathrm{~mol} \%, 1.4 \mathrm{mg})$ and $\mathrm{NH}_{3} \mathrm{BD}_{3}(0.3 \mathrm{mmol}, 10 \mathrm{mg})$ was taken followed by addition of THF $(0.4 \mathrm{~mL}), 1 \mathbf{a}(0.1 \mathrm{mmol}, 10.4 \mu \mathrm{L})$ and $n$-decane $(0.1 \mathrm{mmol}, 19.5 \mu \mathrm{L})$ inside the Ar filled glove box. The reaction mixture was taken out of the glove box and was heated at $60{ }^{\circ} \mathrm{C}$, and the aliquot (3-15) was collected at 2 min intervals, and the yield was monitored by GC-MS. From the plot of the yield of $2 \mathrm{a}$ vs time, the initial rate was determined. The $\mathrm{KIE}_{1}=k_{\mathrm{NH} \cdot \mathrm{BH}} / k_{\mathrm{NH} \cdot \mathrm{BD}}=2.4$ was obtained.

The same procedure was followed for $\mathrm{ND}_{3} \mathrm{BH}_{3}(0.3 \mathrm{mmol}, 10 \mathrm{mg}), \mathrm{ND}_{3} \mathrm{BD}_{3}(0.3 \mathrm{mmol}, 10.5 \mathrm{mg})$, and $\mathrm{KIE}_{2}=k_{\mathrm{NH} \cdot \mathrm{BH}} / k_{\mathrm{ND} \cdot \mathrm{BH}}=1.5$, and overall KIE $=k_{\mathrm{NH} \cdot \mathrm{BH}} / k_{\mathrm{ND} \cdot \mathrm{BD}}=3.2$ were determined.

\begin{tabular}{|c|c|c|c|c|}
\hline & $\mathbf{N H}_{\mathbf{3}} \mathbf{B H}_{\mathbf{3}}$ & $\mathbf{N H}_{\mathbf{3}} \mathbf{B D}_{\mathbf{3}}$ & $\mathbf{N D}_{\mathbf{3}} \mathbf{B H}_{\mathbf{3}}$ & $\mathbf{N D}_{\mathbf{3}} \mathbf{B D}_{\mathbf{3}}$ \\
\hline $\boldsymbol{k}$ & 0.0107 & 0.00440 & 0.00720 & 0.00340 \\
\hline KIE & & 2.4 & 1.5 & 3.20 \\
\hline
\end{tabular}




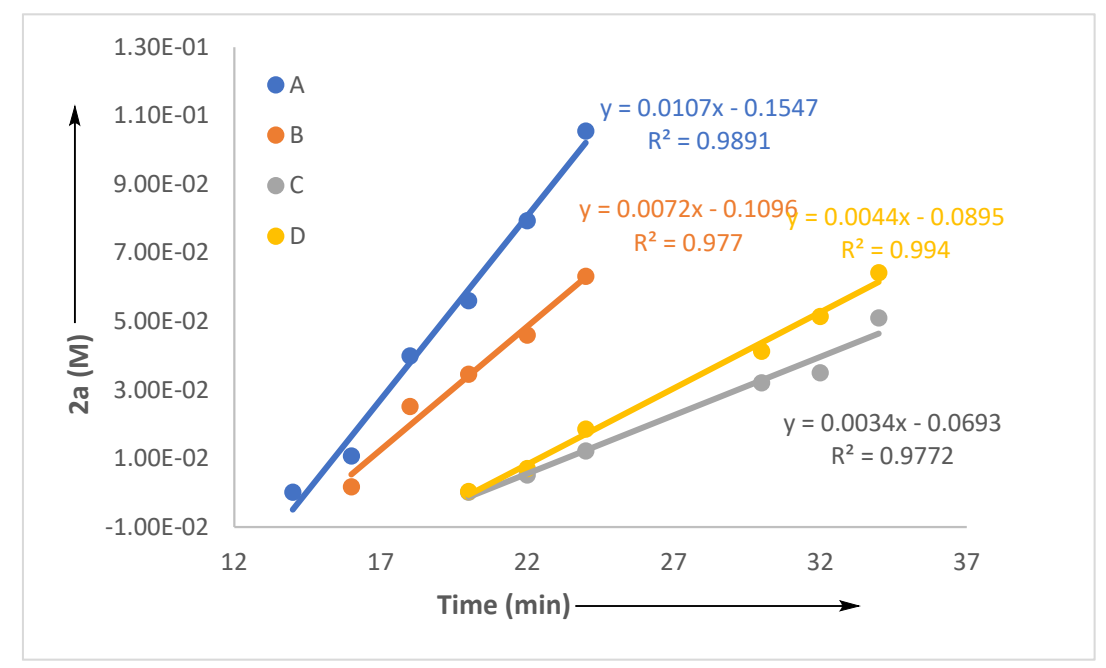

Figure S13. Plot of [2a] vs time for varying concentrations of $\mathrm{A}=\mathrm{NH}_{3} \mathrm{BH}_{3}, \mathrm{~B}=\mathrm{ND}_{3} \mathrm{BH}_{3}, \mathrm{C}=$ $\mathrm{ND}_{3} \mathrm{BD}_{3}$, and $\mathrm{D}=\mathrm{NH}_{3} \mathrm{BD}_{3}$.

\section{7i. Probing the dehydrogenation of $\mathrm{NH}_{3} \mathrm{BH}_{3}$}

In an oven-dried Schlenk tube Mn2 (0.0030 mmol, $1.4 \mathrm{mg}), \mathrm{AB}(0.3 \mathrm{mmol}, 9.3 \mathrm{mg})$ and $\mathrm{PPh}_{3}(0.020$ mmol, $5.3 \mathrm{mg}$ ) were added followed by addition of $n$-hexane $(1 \mathrm{~mL})$ at $60^{\circ} \mathrm{C}$. Outlet of the Schlenk tube was connected with a silicon pipe and the other end of the pipe was connected with the water filled burette. In kinetics hydrogen gas evolution was monitored volumetrically using a burette filled with water. The amount of hydrogen gas generated is calculated using $\mathrm{PV}=\mathrm{nRT}$ equation.

The same experiment was then performed without adding $\mathrm{PPh}_{3}$. Similar rate of hydrogen evolution was observed.

\begin{tabular}{|c|c|c|c|}
\hline \multicolumn{2}{|c|}{ In the presence of external base $\mathbf{P P h}_{\mathbf{3}}$} & \multicolumn{2}{c|}{ In the absence of external base $\mathbf{P P h}_{\mathbf{3}}$} \\
\hline Time (sec) & Volume of $\mathrm{H}_{2}(\mathrm{~mL})$ & Time $(\mathrm{sec})$ & Volume of $\mathrm{H}_{2}(\mathrm{~mL})$ \\
\hline 0 & 0 & 0 & 0 \\
\hline 30 & 0.8 & 30 & 0.7 \\
\hline 60 & 1.6 & 60 & 1.8 \\
\hline 90 & 2.1 & 90 & 2.7 \\
\hline 120 & 2.6 & 120 & 3.1 \\
\hline 150 & 2.9 & 150 & 3.4 \\
\hline 180 & 3.1 & 180 & 3.6 \\
\hline 210 & 3.4 & 211 & 3.8 \\
\hline 240 & 3.5 & 240 & 3.9 \\
\hline 300 & 3.6 & 300 & 3.9 \\
\hline 360 & 3.8 & 391 & 4.1 \\
\hline 420 & 3.9 & 613 & 4.2 \\
\hline
\end{tabular}




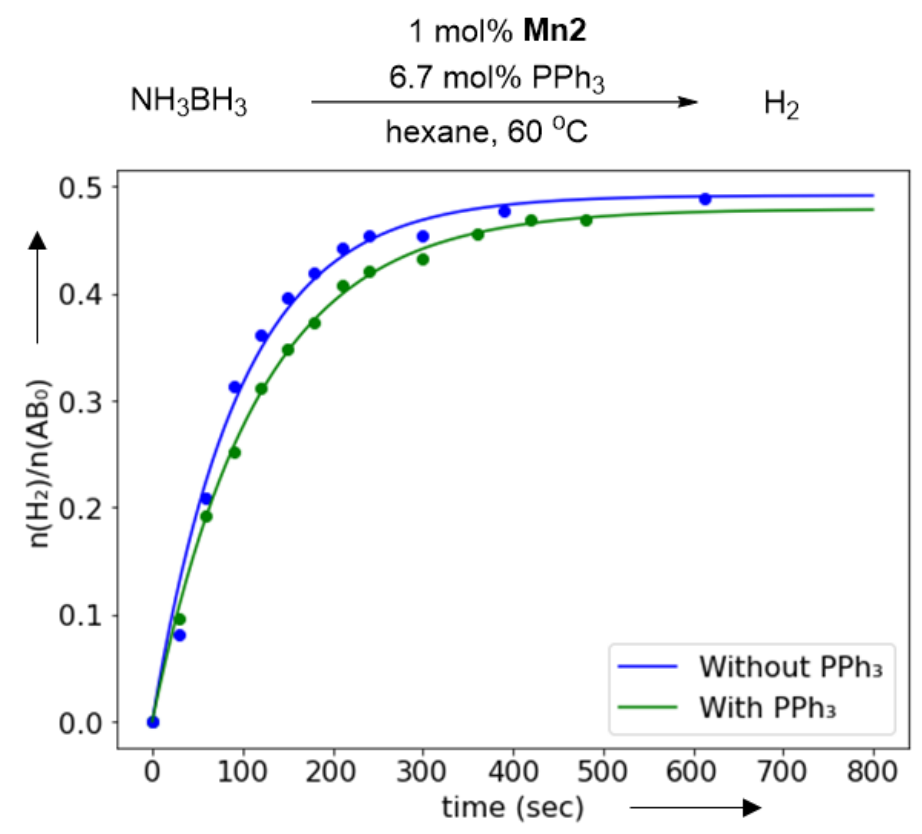

Figure S14. Kinetic monitoring of the Mn2 catalyzed dehydrogenation reaction of $A B$ in the presence and absence of $\mathrm{PPh}_{3}$.

${ }^{11}$ B NMR experiment: In a $\mathrm{N}_{2}$ filled glove box, $\mathrm{NH}_{3} \mathrm{BH}_{3}(0.90 \mathrm{mmol}, 27.8 \mathrm{mg})$, $\mathbf{M n} 2$ catalyst $(0.009$ mmol, $4.167 \mathrm{mg})$, and $\mathrm{PPh}_{3}(0.5 \mathrm{mg}, 0.0019 \mathrm{mmol})$ were taken in a J. Young NMR tube followed by the addition of $0.5 \mathrm{~mL}$ of $\mathrm{C}_{6} \mathrm{D}_{6}$. The NMR tube was sealed and shaken and quickly ${ }^{11} \mathrm{~B}$ NMR of the resulting mixture was taken at room temperature without spinning and data collected at 385 scans.

PBZ

CDB / CTB

Ammoniaborane

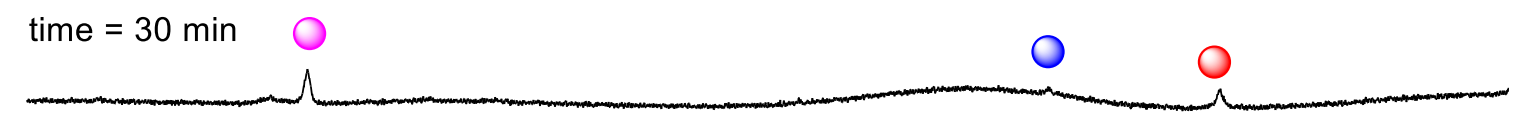

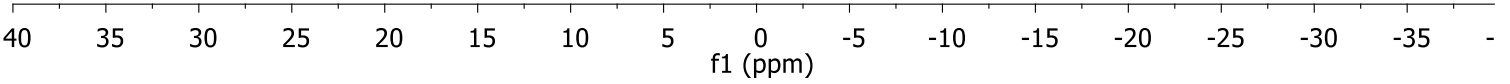

Figure S15: ${ }^{11} \mathrm{~B}$ NMR in $\mathrm{C}_{6} \mathrm{D}_{6}$ in the presence of $\mathbf{M n 2}, \mathbf{A B}$, and $\mathbf{P P h}_{3}$, Where $\mathrm{CDB}=$ Cyclodiaminoborane, $\mathrm{CTB}=$ Cyclotriaminoborane, $\mathrm{PBZ}=$ Polyborazine . 
phenylmethanaminium chloride(2'a $)^{5}$ : Yield $=89 \%(32 \mathrm{mg}, 0.22 \mathrm{mmol}) .{ }^{1} \mathbf{H}$ NMR $\left(400 \mathrm{MHz}, \mathrm{D}_{2} \mathrm{O}\right)$

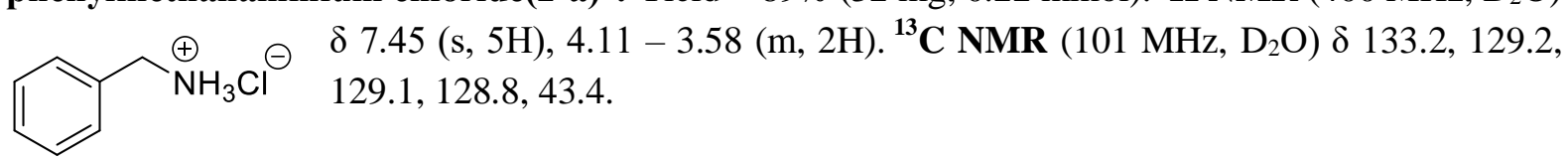
p-tolylmethanaminium chloride(2'b) ${ }^{5}$ : Yield $=98 \%$ (39 mg, $\left.0.24 \mathrm{mmol}\right) .{ }^{1} \mathbf{H}$ NMR $\left(400 \mathrm{MHz}, \mathrm{D}_{2} \mathrm{O}\right)$ $\begin{array}{ll}\mathrm{NH}_{3} \mathrm{Cl}^{\ominus} & \delta 7.18(\mathrm{~d}, J=7.8 \mathrm{~Hz}, 4 \mathrm{H}), 3.99(\mathrm{~s}, 2 \mathrm{H}), 2.20(\mathrm{~s}, 3 \mathrm{H}) .{ }^{13} \mathbf{C} \mathbf{N M R}(101 \mathrm{MHz} \text {, } \\ \left.\mathrm{D}_{2} \mathrm{O}\right) \delta 139.6,129.9,129.8,128.9,43.1,20.3 .\end{array}$ m-tolylmethanaminium chloride (2'c) $)^{5}:$ Yield $=96 \%(38 \mathrm{mg}, 0.24 \mathrm{mmol}) .{ }^{1} \mathbf{H}$ NMR $\left(400 \mathrm{MHz}, \mathrm{D}_{2} \mathrm{O}\right)$ $\overbrace{}^{\oplus} \ominus \delta 7.33(\mathrm{t}, J=7.7 \mathrm{~Hz}, 1 \mathrm{H}), 7.23(\mathrm{dd}, J=17.7,7.3 \mathrm{~Hz}, 3 \mathrm{H}), 4.08(\mathrm{~s}, 2 \mathrm{H}), 2.31(\mathrm{~s}$, $3 \mathrm{H}) .{ }^{13} \mathbf{C}$ NMR $\left(101 \mathrm{MHz}, \mathrm{D}_{2} \mathrm{O}\right) \delta 139.4,133.0,129.7,129.3,129.2,125.7$, 43.3, 20.4.

o-tolylmethanaminium chloride(2'd) ${ }^{5}$ : Yield $=93 \%(37 \mathrm{mg}, 0.23 \mathrm{mmol}) .{ }^{1} \mathbf{H}$ NMR $\left(400 \mathrm{MHz}, \mathrm{D}_{2} \mathrm{O}\right)$ $\begin{array}{ll}\mathrm{NH}_{3} \mathrm{Cl}^{\ominus} & \delta 7.27(\mathrm{~s}, 4 \mathrm{H}), 4.13(\mathrm{~s}, 2 \mathrm{H}), 2.31(\mathrm{~s}, 3 \mathrm{H}) .{ }^{13} \mathrm{C} \mathbf{N M R}\left(126 \mathrm{MHz}, \mathrm{D}_{2} \mathrm{O}\right) \delta 137.1 \text {, } \\ & 131.4,130.8,129.2,128.9,126.5,40.5,18.0 .\end{array}$

(3-methoxyphenyl)methanaminium chloride(2'e $)^{5}$ : Yield $=92 \%(40.1 \mathrm{mg}, 0.22 \mathrm{mmol}) .{ }^{1} \mathbf{H}$ NMR MeO $\stackrel{P}{A}^{\ominus}\left(500 \mathrm{MHz}, \mathrm{D}_{2} \mathrm{O}\right) \delta 7.39(\mathrm{~s}, 1 \mathrm{H}), 7.01(\mathrm{~s}, 3 \mathrm{H}), 4.11(\mathrm{~s}, 2 \mathrm{H}), 3.81(\mathrm{~s}, 3 \mathrm{H})$. ${ }^{13}$ C NMR (126 MHz, D $\left.2 \mathrm{O}\right) \delta 158.8,134.5,130.1,120.9,114.1,113.9$, 55.0, 42.9 .

(4-methoxyphenyl)methanaminium(2' f) ${ }^{5}$ : Yield $=85 \%$ (37 mg, $\left.0.21 \mathrm{mmol}\right) .{ }^{\mathbf{1}} \mathbf{H}$ NMR (400 MHz, $\begin{array}{ll}\mathrm{NH}_{3} \mathrm{Cl}^{\ominus} & \begin{array}{l}\left.\mathrm{D}_{2} \mathrm{O}\right) \delta 7.23(\mathrm{~d}, J=6.9 \mathrm{~Hz}, 2 \mathrm{H}), 6.86(\mathrm{~d}, J=6.5 \mathrm{~Hz}, 2 \mathrm{H}), 3.93(\mathrm{~s}, 2 \mathrm{H}), 3.66 \\ (\mathrm{~s}, 3 \mathrm{H}) .{ }^{13} \mathbf{C N M R}\left(101 \mathrm{MHz}, \mathrm{D}_{2} \mathrm{O}\right) \delta 159.3,130.5,125.7,114.5,55.4,42.9 .\end{array}\end{array}$

(3-chlorophenyl)methanaminium chloride(2'g) ${ }^{5}$ : Yield $=71 \%(31.5 \mathrm{mg}, 0.18 \mathrm{mmol}) .{ }^{1} \mathbf{H}$ NMR (400<smiles>[NH3+]Cc1cccc(Cl)c1</smiles>
$\left.\mathrm{MHz}, \mathrm{D}_{2} \mathrm{O}\right) \delta 7.35(\mathrm{~d}, J=1.5 \mathrm{~Hz}, 1 \mathrm{H}), 7.33-7.32(\mathrm{~m}, 1 \mathrm{H}), 7.31-7.28(\mathrm{~m}$, $1 \mathrm{H}), 7.24(\mathrm{dd}, J=5.3,3.4 \mathrm{~Hz}, 1 \mathrm{H}), 4.03(\mathrm{~s}, 2 \mathrm{H}) .{ }^{13} \mathbf{C} \mathbf{N M R}\left(101 \mathrm{MHz}, \mathrm{D}_{2} \mathrm{O}\right)$ $\delta 134.7,134.2,130.7,129.2,128.8,127.2,42.7$.

(4-bromophenyl)methanaminium chloride(2'h $)^{6}$ : Yield = 73\%, (41 mg, $\left.0.18 \mathrm{mmol}\right) .{ }^{1} \mathbf{H}$ NMR (400

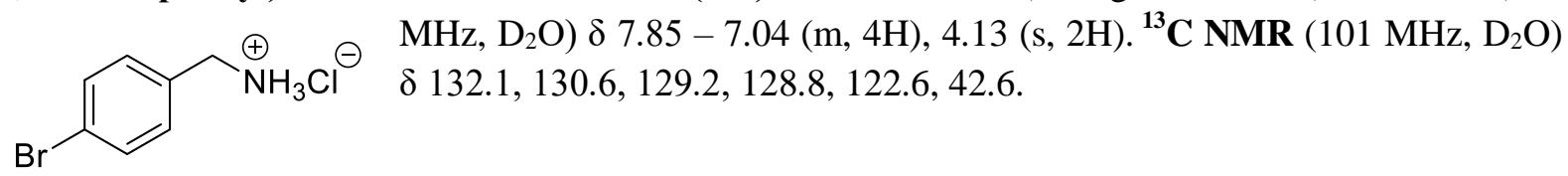

(4-fluorophenyl)methanaminium chloride(2'i) ${ }^{6}$ : Yield $=85 \%$ (35 mg, $\left.0.21 \mathrm{mmol}\right) .{ }^{1} \mathbf{H}$ NMR (400 $\begin{array}{ll}\mathrm{NH}_{3} \mathrm{Cl} & \begin{array}{l}\left.\mathrm{MHz}, \mathrm{D}_{2} \mathrm{O}\right) \delta 7.46(\mathrm{~s}, 2 \mathrm{H}), 7.20(\mathrm{~s}, 2 \mathrm{H}), 4.15(\mathrm{~s}, 2 \mathrm{H}) .{ }^{13} \mathbf{C} \mathbf{N M R}(101 \mathrm{MHz}, \\ \left.\mathrm{D}_{2} \mathrm{O}\right) \delta 162.8(\mathrm{~d}, J=246.4 \mathrm{~Hz}), 131.0(\mathrm{~d}, J=6.1 \mathrm{~Hz}), 128.90,116.0(\mathrm{~d}, J= \\ 22.2 \mathrm{~Hz}), 42.6 .\end{array}\end{array}$

(2-fluorophenyl)methanaminium(2'j) ${ }^{6}$ : Yield $=91 \%(36.8 \mathrm{mg}, 0.22 \mathrm{mmol}) .{ }^{\mathbf{1}} \mathbf{H}$ NMR $(400 \mathrm{MHz}$,<smiles>Fc1ccccc1C[18O][Na]</smiles>
$\left.\mathrm{D}_{2} \mathrm{O}\right) \delta 7.48(\mathrm{~d}, J=6.4 \mathrm{~Hz}, 1 \mathrm{H}), 7.24(\mathrm{dd}, J=20.3,8.4 \mathrm{~Hz}, 3 \mathrm{H}), 4.20(\mathrm{~s}, 2 \mathrm{H})$.

${ }^{13} \mathrm{C}$ NMR $\left(126 \mathrm{MHz}, \mathrm{D}_{2} \mathrm{O}\right) \delta 160.9(\mathrm{~d}, J=252.0 \mathrm{~Hz}), 131.7(\mathrm{~d}, J=63.0 \mathrm{~Hz})$, $131.2(\mathrm{~d}, J=3.4 \mathrm{~Hz}), 125.0(\mathrm{~d}, J=6.3 \mathrm{~Hz}), 119.8(\mathrm{~d}, J=15.1 \mathrm{~Hz}), 115.8(\mathrm{~d}, J=$ 21.4), 37.4 . 
(3-fluorophenyl)methenamine(2'k) ${ }^{6}$ : Yield $=81 \%(33 \mathrm{mg}, 0.20 \mathrm{mmol}) .{ }^{1} \mathbf{H} \mathbf{N M R}\left(400 \mathrm{MHz}, \mathrm{D}_{2} \mathrm{O}\right) \delta$<smiles>[NH3+]Cc1cccc(F)c1</smiles>
$7.47(\mathrm{dd}, J=13.9,7.8 \mathrm{~Hz}, 1 \mathrm{H}), 7.26(\mathrm{~d}, J=7.7 \mathrm{~Hz}, 1 \mathrm{H}), 7.23-7.18(\mathrm{~m}, 2 \mathrm{H})$, $4.19(\mathrm{~s}, 2 \mathrm{H}) .{ }^{13} \mathbf{C} \mathbf{N M R}\left(126 \mathrm{MHz}, \mathrm{D}_{2} \mathrm{O}\right) \delta 162.6(\mathrm{~d}, \mathrm{~J}=239 \mathrm{~Hz}), 135.0(\mathrm{~d}, \mathrm{~J}=$ $7.56 \mathrm{~Hz}), 131.1(\mathrm{~d}, J=8.82 \mathrm{~Hz}), 124.6(\mathrm{~d}, J=2.52 \mathrm{~Hz}), 116.0(\mathrm{~d}, J=18.9 \mathrm{~Hz})$, $115.7(\mathrm{~d}, J=22.86 \mathrm{~Hz}), 42.72$.

(4-iodophenyl)methanaminium chloride(2'l) ${ }^{7}$ : Yield = 62\% (42 mg, $\left.0.15 \mathrm{mmol}\right){ }^{1}{ }^{1} \mathrm{H}$ NMR (400 $\begin{array}{ll}\mathrm{NH}_{3} \mathrm{Cl}^{\ominus} & \begin{array}{l}\left.\mathrm{MHz}, \mathrm{D}_{2} \mathrm{O}\right) \delta 7.70(\mathrm{~d}, J=7.1 \mathrm{~Hz}, 1 \mathrm{H}), 7.32(\mathrm{~s}, 2 \mathrm{H}), 7.06(\mathrm{~d}, J=9.5 \mathrm{~Hz}, 1 \mathrm{H}) \\ 128.8,42.5 .\end{array}\end{array}$

(1-oxo-1,3-dihydroisobenzofuran-5-yl)methanaminium chloride(2' $\mathbf{m})^{8}$ : Yield $=50 \%(25 \mathrm{mg}, 0.125$ $\begin{array}{ll}\mathrm{O} & \begin{array}{l}\mathrm{mmol}) .{ }^{1} \mathbf{H} \mathbf{N M R}\left(400 \mathrm{MHz}, \mathrm{D}_{2} \mathrm{O}\right) \delta 7.91(\mathrm{~d}, J=7.8 \mathrm{~Hz}, 1 \mathrm{H}), 7.79-7.54(\mathrm{~m}, \\ 2 \mathrm{H}), 5.44(\mathrm{~s}, 2 \mathrm{H}), 4.31(\mathrm{~s}, 2 \mathrm{H}) .{ }^{13} \mathbf{C} \mathbf{N M R}\left(126 \mathrm{MHz}, \mathrm{D}_{2} \mathrm{O}\right) \delta 233.9,226.2, \\ 208.3,189.4,185.8,185.1,182.7,131.1,102.8 .\end{array}\end{array}$ 1-(4-(aminomethyl)phenyl)ethan-1-ol( 2'n) ${ }^{\mathbf{9}}$ :Yield = 76\% (0.37 mmol, $\left.56.5 \mathrm{mg}\right) .{ }^{\mathbf{1}} \mathbf{H}$ NMR (400<smiles>CC(O)c1ccc(CN)cc1</smiles>
$\left.\mathrm{MHz}, \mathrm{CDCl}_{3}\right) \delta 7.37(\mathrm{dd}, J=20.3,7.9 \mathrm{~Hz}, 2 \mathrm{H}), 7.30-7.26(\mathrm{~m}, 2 \mathrm{H}), 4.91(\mathrm{~d}, J$ $=6.4 \mathrm{~Hz}, 1 \mathrm{H}), 3.91(\mathrm{~s}, 2 \mathrm{H}), 1.48(\mathrm{~d}, J=6.3 \mathrm{~Hz}, 3 \mathrm{H}) \cdot{ }^{13} \mathbf{C ~ N M R}(101 \mathrm{MHz}$, $\left.\mathrm{CDCl}_{3}\right) \delta 146.6,135.2,128.6,126.4,70.0,52.9,25.4$.

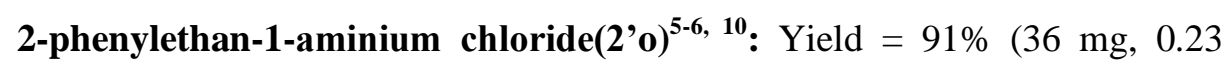<smiles>[NH3+]CCc1ccccc1</smiles>
mmol). ${ }^{1} \mathbf{H}$ NMR $\left(500 \mathrm{MHz}, \mathrm{D}_{2} \mathrm{O}\right) \delta$ 7.65-7.54 (m, $\left.J=17.9,7.4 \mathrm{~Hz}, 5 \mathrm{H}\right), 4.70$ $(\mathrm{s}, 2 \mathrm{H}), 3.22(\mathrm{t}, J=7.3 \mathrm{~Hz}, 2 \mathrm{H}), 2.94(\mathrm{t}, J=7.3 \mathrm{~Hz}, 2 \mathrm{H}) .{ }^{13} \mathbf{C} \mathbf{N M R}(126 \mathrm{MHz}$, $\left.\mathrm{D}_{2} \mathrm{O}\right) \delta 136.6,129.0,128.8,127.3,40.7,32.8$.

3-phenylpropan-1-aminium chloride(2'p) ${ }^{11}$ : Yield $=50 \%$ (21 mg, $\left.0.12 \mathrm{mmol}\right) .{ }^{1} \mathbf{H}$ NMR (400 MHz,<smiles>[NH3+]CCCc1ccccc1</smiles>
$\left.\mathrm{D}_{2} \mathrm{O}\right) \delta 7.39(\mathrm{t}, J=7.3 \mathrm{~Hz}, 2 \mathrm{H}), 7.35-7.20(\mathrm{~m}, 3 \mathrm{H}), 3.00(\mathrm{t}, J=7.1 \mathrm{~Hz}, 2 \mathrm{H})$, $2.73(\mathrm{t}, J=7.6 \mathrm{~Hz}, 2 \mathrm{H}), 2.03-1.91(\mathrm{~m}, 2 \mathrm{H}) .{ }^{13} \mathbf{C ~ N M R}\left(101 \mathrm{MHz}, \mathrm{D}_{2} \mathrm{O}\right) \delta$ $141.0,128.8,128.5,126.5,39.3,31.8,28.6$.

2-(p-tolyl)ethan-1-aminium chloride(2'q): ${ }^{12}$ Yield = 81\% (35 mg, $\left.0.20 \mathrm{mmol}\right) .{ }^{1} \mathbf{H}$ NMR (400 MHz, $\begin{array}{ll}\stackrel{\oplus}{\oplus} \mathrm{NH}_{3} \mathrm{Cl} & \left.\mathrm{D}_{2} \mathrm{O}\right) \delta 7.08(\mathrm{~s}, 4 \mathrm{H}), 3.09(\mathrm{~d}, J=6.9 \mathrm{~Hz}, 2 \mathrm{H}), 2.79(\mathrm{~d}, J=6.5 \mathrm{~Hz}, 2 \mathrm{H}), 2.16 \\ (\mathrm{~s}, 3 \mathrm{H}) .{ }^{13} \mathbf{C} \mathbf{N M R}\left(101 \mathrm{MHz}, \mathrm{D}_{2} \mathrm{O}\right) \delta 137.4,133.6,129.6,128.9,40.9,32.4 \text {, } \\ 20.1 .\end{array}$

2-(2-methoxyphenyl)ethan-1-aminium chloride(2'r $)^{5}$ : Yield = 85\% (40 mg, $\left.0.21 \mathrm{mmol}\right)$.

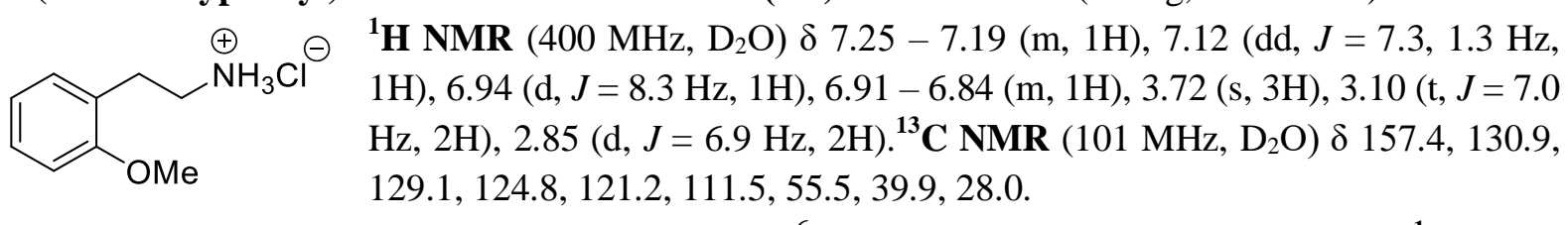

(4-methoxyphenyl)methanaminium chloride(2's) ${ }^{6}$ : Yield $=96 \%(41.8 \mathrm{mg}, 0.24 \mathrm{mmol}) .{ }^{1} \mathbf{H}$ NMR<smiles>COc1ccc(CC[NH3+])cc1</smiles>
$\left(400 \mathrm{MHz}, \mathrm{D}_{2} \mathrm{O}\right) \delta 7.13(\mathrm{~d}, J=8.0 \mathrm{~Hz}, 2 \mathrm{H}), 6.85(\mathrm{~d}, J=7.9 \mathrm{~Hz}, 2 \mathrm{H})$, $3.67(\mathrm{~s}, 3 \mathrm{H}), 3.10(\mathrm{t}, J=7.1 \mathrm{~Hz}, 2 \mathrm{H}), 2.79(\mathrm{t}, J=7.1 \mathrm{~Hz}, 2 \mathrm{H}) .{ }^{13} \mathbf{C} \mathbf{N M R}$ $\left(101 \mathrm{MHz}, \mathrm{D}_{2} \mathrm{O}\right) \delta 158.0,130.2,129.2,114.5,55.4,40.9,32.0$. 
2-(3,4-dimethoxyphenyl)ethan-1-aminium(2’t $)^{6}$ : Yield $=90 \%$ (49.1 mg, $\left.0.22 \mathrm{mmol}\right) .{ }^{\mathbf{1}} \mathbf{H}$ NMR (400<smiles>COc1ccc(CC[NH3+])cc1OC</smiles>
$\left.\mathrm{MHz}, \mathrm{D}_{2} \mathrm{O}\right) \delta 6.75(\mathrm{~d}, J=8.1 \mathrm{~Hz}, 1 \mathrm{H}), 6.72(\mathrm{~s}, 1 \mathrm{H}), 6.66(\mathrm{~d}, J=7.9 \mathrm{~Hz}$, $1 \mathrm{H}), 3.61(\mathrm{~s}, J=7.6 \mathrm{~Hz}, 6 \mathrm{H}), 3.04(\mathrm{t}, J=7.1 \mathrm{~Hz}, 2 \mathrm{H}), 2.71(\mathrm{t}, J=7.1$ $\mathrm{Hz}, 2 \mathrm{H}), 1.85(\mathrm{~s}, 1 \mathrm{H}) .{ }^{13} \mathrm{C}$ NMR $\left(101 \mathrm{MHz}, \mathrm{D}_{2} \mathrm{O}\right) \delta 141.0,128.8,128.5$, $126.5,39.3,31.8,28.6$.

2-(3-fluorophenyl)ethan-1-aminium chloride(2'u) ${ }^{10}$ : Yield $=81 \%(36 \mathrm{mg}, 0.20 \mathrm{mmol}) .{ }^{1} \mathbf{H}$ NMR<smiles></smiles>
$\left(400 \mathrm{MHz}, \mathrm{D}_{2} \mathrm{O}\right) \delta 7.40(\mathrm{~d}, J=6.6 \mathrm{~Hz}, 1 \mathrm{H}), 7.25-6.99(\mathrm{~m}, 4 \mathrm{H}), 3.29(\mathrm{t}, J=$ $6.8 \mathrm{~Hz}, 2 \mathrm{H}), 3.01(\mathrm{t}, J=6.6 \mathrm{~Hz}, 3 \mathrm{H}) .{ }^{13} \mathbf{C} \mathbf{~ N M R}\left(101 \mathrm{MHz}, \mathrm{D}_{2} \mathrm{O}\right) \delta 162.8(\mathrm{~d}$, $\mathrm{J}=245 \mathrm{~Hz}), 139.2(\mathrm{~d}, \mathrm{~J}=7.07 \mathrm{~Hz}), 130.7(\mathrm{~d}, \mathrm{~J}=8.08 \mathrm{~Hz}), 124.8,115.6(\mathrm{~d}, \mathrm{~J}$ $=22.22 \mathrm{~Hz}), 114.1(\mathrm{~d}, \mathrm{~J}=21.21 \mathrm{~Hz}), 40.6,32.6$.

2-(4-fluorophenyl)ethan-1-aminium chloride(2'v $)^{10}$ : Yield $=81 \%(35.7 \mathrm{mg}, 0.20 \mathrm{mmol}) .{ }^{\mathbf{1}} \mathbf{H}$ NMR<smiles>Fc1ccc(CC[N]Cl)cc1</smiles>
$\left(400 \mathrm{MHz}, \mathrm{D}_{2} \mathrm{O}\right) \delta 7.30(\mathrm{dd}, J=8.4,5.6 \mathrm{~Hz}, 2 \mathrm{H}), 7.11(\mathrm{t}, J=8.8 \mathrm{~Hz}, 2 \mathrm{H})$, $3.24(\mathrm{t}, J=7.3 \mathrm{~Hz}, 1 \mathrm{H}), 2.96(\mathrm{t}, J=7.2 \mathrm{~Hz}, 1 \mathrm{H}) .{ }^{13} \mathbf{C} \mathbf{~ N M R}\left(126 \mathrm{MHz}, \mathrm{D}_{2} \mathrm{O}\right)$ $\delta 161.6(\mathrm{~d}, \mathrm{~J}=243.2 \mathrm{~Hz}), 132.3(\mathrm{~d}, \mathrm{~J}=2.52 \mathrm{~Hz}), 130.4(\mathrm{~d}, \mathrm{~J}=7.6 \mathrm{~Hz}), 115.6(\mathrm{~d}$, $\mathrm{J}=21.42 \mathrm{~Hz}), 40.67,32.00 .0$

2-(4-chlorophenyl)ethan-1-aminium chloride (2'w ${ }^{10}$ : Yield $=61 \%(29.5 \mathrm{mg}, 0.15 \mathrm{mmol}) .{ }^{\mathbf{1}} \mathbf{H} \mathbf{~ N M R}$ $\begin{array}{ll}\mathrm{NH}_{3} \mathrm{Cl}^{\ominus} & \begin{array}{l}\left(400 \mathrm{MHz}, \mathrm{D}_{2} \mathrm{O}\right) \delta 7.25(\mathrm{~d}, J=8.4 \mathrm{~Hz}, 1 \mathrm{H}), 7.13(\mathrm{~d}, J=8.4 \mathrm{~Hz}, 1 \mathrm{H}), 3.11 \\ (\mathrm{t}, J=7.3 \mathrm{~Hz}, 1 \mathrm{H}), 2.82(\mathrm{t}, J=7.3 \mathrm{~Hz}, 1 \mathrm{H}) .{ }^{\mathbf{1 3}} \mathbf{C} \mathbf{N M R}\left(126 \mathrm{MHz}, \mathrm{D}_{2} \mathrm{O}\right) \delta\end{array} \\ \begin{array}{l}135.2,132.3,130.3,128.8,40.5,32.1 .\end{array}\end{array}$

2-(4-bromophenyl)ethan-1-aminium chloride(2'x $)^{10}$ : Yield $=65 \%$ (39 mg, $\left.0.164 \mathrm{mmol}\right) .{ }^{1} \mathbf{H}$ NMR

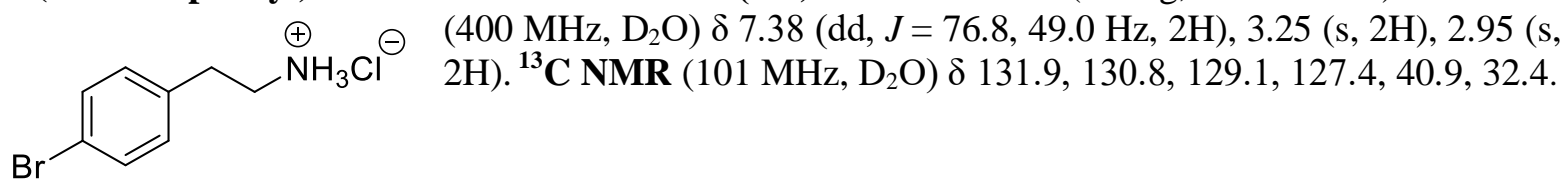
2,2'-(1,4-phenylene)bis(ethan-1-aminium) chloride(2'y ${ }^{13}$ : Yield $=54 \%(32 \mathrm{mg}, 0.135 \mathrm{mmol}) .{ }^{\mathbf{1}} \mathbf{H}$

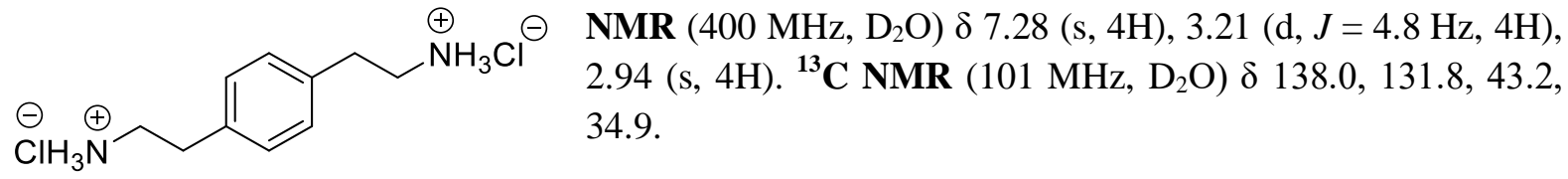

2-(naphthalen-1-yl)ethan-1-amine(2'z) ${ }^{14}$ : Yield $=72 \%(37 \mathrm{mg}, 0.17 \mathrm{mmol}) .{ }^{\mathbf{1}} \mathbf{H}$ NMR $(400 \mathrm{MHz}$, $\begin{array}{ll}\stackrel{\mathrm{NH}}{3} \mathrm{Cl}^{\ominus} & \begin{array}{l}\left.\mathrm{D}_{2} \mathrm{O}\right) \delta 7.92-7.65(\mathrm{~m}, 3 \mathrm{H}), 7.43(\mathrm{dd}, J=14.5,6.3 \mathrm{~Hz}, 2 \mathrm{H}), 7.37-7.21(\mathrm{~m}, \\ 2 \mathrm{H}), 3.25(\mathrm{t}, J=5.2 \mathrm{~Hz}, 2 \mathrm{H}), 3.18(\mathrm{t}, J=3.0 \mathrm{~Hz}, 2 \mathrm{H}) .{ }^{\mathbf{1 3}} \mathbf{C} \mathbf{N M R}(126 \mathrm{MHz},\end{array} \\ \begin{array}{l}\left.\mathrm{D}_{2} \mathrm{O}\right) \delta 133.8,132.7,131.2,129.1,128.1,127.5,126.9,126.4,126.0,123.2, \\ 40.0,30.3 .\end{array}\end{array}$

Hexane-1,6-diaminium chloride(2'za) ${ }^{15}$ : Yield $=95 \%(44.5 \mathrm{mg}, 0.237 \mathrm{mmol}) .{ }^{1} \mathbf{H}$ NMR $(400 \mathrm{MHz}$, $\stackrel{\ominus}{\mathrm{ClH}_{3}} \mathrm{~N}^{\oplus} \mathrm{NH}_{3}^{\oplus} \mathrm{Cl}^{\ominus}$

$\left.\mathrm{D}_{2} \mathrm{O}\right) \delta 2.83(\mathrm{dd}, J=55.3,5.6 \mathrm{~Hz}, 4 \mathrm{H}), 1.50(\mathrm{~d}, J=51.3 \mathrm{~Hz}, 4 \mathrm{H})$ $1.24(\mathrm{~d}, J=56.7 \mathrm{~Hz}, 4 \mathrm{H}) .{ }^{13} \mathrm{C} \mathbf{N M R}\left(101 \mathrm{MHz}, \mathrm{D}_{2} \mathrm{O}\right) \delta 41.0,28.1$, 26.7 . 
Dibenzylamine(3a) ${ }^{14}$ : Yield $=97 \%(24 \mathrm{mg}, 0.12 \mathrm{mmol}) .{ }^{1} \mathbf{H}$ NMR $\left(400 \mathrm{MHz}, \mathrm{CDCl}_{3}\right): \delta 7.38-7.29$<smiles>c1ccc(CNCc2ccccc2)cc1</smiles>
$(\mathrm{m}, 4 \mathrm{H}), 7.25(\mathrm{dd}, J=6.3,4.6, \mathrm{~Hz}, 2 \mathrm{H}), 3.81(\mathrm{~s}, 2 \mathrm{H}), 1.61(\mathrm{~s}, 1 \mathrm{H}) .{ }^{13} \mathrm{C}$ NMR (101 MHz, $\left.\mathrm{CDCl}_{3}\right): \delta 140.5,128.5,128.3,127.1,53.3$.

Bis(3-methyl benzyl)amine(3b) $)^{16}$ : Yield $=98 \%(27.5 \mathrm{mg}, 0.18 \mathrm{mmol}) .{ }^{1} \mathbf{H}$ NMR $\left(500 \mathrm{MHz}, \mathrm{CDCl}_{3}\right)$ :<smiles>Cc1cccc(CNCc2cccc(C)c2)c1</smiles>
$\delta 7.34(\mathrm{t}, J=7.5 \mathrm{~Hz}, 2 \mathrm{H}), 7.29-7.24(\mathrm{~m}, 4 \mathrm{H}), 7.19(\mathrm{~d}, J=7.4 \mathrm{~Hz}, 2 \mathrm{H})$, $3.90(\mathrm{~s}, 6 \mathrm{H}), 2.47$ (s, 4H), $1.84(\mathrm{~s}, 1 \mathrm{H}) .{ }^{13} \mathbf{C} \mathbf{N M R}\left(101 \mathrm{MHz}, \mathrm{CDCl}_{3}\right): \delta$ $140.2,138.1,129.1,128.4,127.8,125.3,53.3,21.5$.

Bis(3-chlorobenzyl)amine(3c) ${ }^{7}$ : Yield $=88 \%(24.8 \mathrm{mg}, 0.11 \mathrm{mmol}) .{ }^{1} \mathbf{H}$ NMR $\left(400 \mathrm{MHz}, \mathrm{CDCl}_{3}\right) \delta$<smiles>Cc1ccc(CNCc2ccc(C)cc2)cc1</smiles>
$7.25(\mathrm{~d}, J=7.9 \mathrm{~Hz}, 4 \mathrm{H}), 7.16(\mathrm{~d}, J=7.9 \mathrm{~Hz}, 4 \mathrm{H}), 3.78(\mathrm{~s}, 4 \mathrm{H}), 2.36(\mathrm{~s}$, $6 \mathrm{H}) .{ }^{13} \mathbf{C}$ NMR $\left(101 \mathrm{MHz}, \mathrm{CDCl}_{3}\right) \delta 137.4,136.6,129.2,128.2,77.5$, $77.2,76.9,52.9,21.2$.

Bis(4-methoxybenzyl)amine(3d) ${ }^{7}$ : Yield $=93 \%(30 \mathrm{mg}, 0.116 \mathrm{mmol}) .{ }^{1} \mathbf{H}$ NMR $\left(500 \mathrm{MHz}, \mathrm{CDCl}_{3}\right)$ :<smiles>COc1cccc(CNCc2cccc(OC)c2)c1</smiles>
$\delta 7.25(\mathrm{~d}, \mathrm{~J}=8.7 \mathrm{~Hz}, 2 \mathrm{H}), 7.25(\mathrm{~d}, \mathrm{~J}=8.7 \mathrm{~Hz}, 2 \mathrm{H}), 6.87(\mathrm{~d}, \mathrm{~J}=$ $8.5 \mathrm{~Hz}, 2 \mathrm{H}), 6.87(\mathrm{~d}, \mathrm{~J}=8.5 \mathrm{~Hz}, 2 \mathrm{H}), 3.80(\mathrm{~s}, 6 \mathrm{H}), 3.73(\mathrm{~s}, 4 \mathrm{H})$, $1.63(\mathrm{~s}, 1 \mathrm{H}) .{ }^{13} \mathrm{C}$ NMR $\left(101 \mathrm{MHz}, \mathrm{CDCl}_{3}\right) \delta 159.9,140.9$, 129.6, 120.8, 113.9, 112.9, 5.33, 52.6.

Bis(3-fluorobenzyl)amine(3e) ${ }^{7}$ : Yield $=72 \%(42 \mathrm{mg}, 0.18 \mathrm{mmol}) .{ }^{1} \mathbf{H}$ NMR $\left(400 \mathrm{MHz}, \mathrm{CDCl}_{3}\right): \delta$<smiles>Fc1cccc(CNCc2cccc(F)c2)c1</smiles>
$7.30-7.23(\mathrm{~m}, 2 \mathrm{H}), 7.10-7.06(\mathrm{~m}, 6 \mathrm{H}), 6.96-6.91(\mathrm{~m}, 3 \mathrm{H}), 3.78(\mathrm{~s}$, 4H), $1.65(\mathrm{~s}, 1 \mathrm{H}) .{ }^{13} \mathbf{C}$ NMR $\left(101 \mathrm{MHz}, \mathrm{CDCl}_{3}\right): \delta 163.2(\mathrm{~d}, \mathrm{~J}=252.5$ $\mathrm{Hz}), 143.0(\mathrm{~d}, \mathrm{~J}=8.08 \mathrm{~Hz}), 130.0(\mathrm{~d}, \mathrm{~J}=8.08 \mathrm{~Hz}), 123.7,115.0(\mathrm{~d}, \mathrm{~J}$ $=21 \mathrm{~Hz}), 114.0(\mathrm{~d}, \mathrm{~J}=21.21 \mathrm{~Hz}), 52.6,29.8$.

Bis(4-fluorobenzyl)amine(3f) ${ }^{7}$ : Yield $=72 \%(42 \mathrm{mg}, 0.18 \mathrm{mmol}) .{ }^{1} \mathbf{H}$ NMR $\left(400 \mathrm{MHz}, \mathrm{CDCl}_{3}\right): \delta$<smiles>Fc1ccc(CNCc2ccc(F)cc2)cc1</smiles>
$7.30(\mathrm{dd}, J=8.5,5.6 \mathrm{~Hz}, 4 \mathrm{H}), 7.02(\mathrm{t}, J=8.7 \mathrm{~Hz}, 4 \mathrm{H}), 3.76(\mathrm{~s}, 4 \mathrm{H})$, $1.63(\mathrm{~s}, 1 \mathrm{H}) .{ }^{13} \mathrm{C}$ NMR $\left(101 \mathrm{MHz}, \mathrm{CDCl}_{3}\right): \delta 162.0(\mathrm{~d}, \mathrm{~J}=252.5 \mathrm{~Hz})$, $136.0,129.8(\mathrm{~d}, \mathrm{~J}=10.1 \mathrm{~Hz}), 115.3(\mathrm{~d}, \mathrm{~J}=20.2 \mathrm{~Hz}), 52.5$.

Bis(3-chlorobenzyl)amine(3g) ${ }^{16}$ : Yield $=55 \%(36 \mathrm{mg}, 0.13 \mathrm{mmol}) .{ }^{1} \mathbf{H}$ NMR $\left(400 \mathrm{MHz}, \mathrm{CDCl}_{3}\right) \delta$<smiles>Clc1cccc(CNCc2cccc(Cl)c2)c1</smiles>
7.34 (s, 2H), 7.23-7.21 (dd, 6H), $3.76(\mathrm{~s}, 4 \mathrm{H}), 1.63(\mathrm{~s}, 1 \mathrm{H}) .{ }^{\mathbf{1 3}} \mathbf{C}$ NMR $\left(101 \mathrm{MHz}, \mathrm{CDCl}_{3}\right) \delta 142.3,134.4,129.8,128.3,127.3,126.3,77.4$, $77.1,76.8,52.6$.

Bis(4-bromobenzyl)amine(3h) ${ }^{7}$ : Yield $=91 \%(39 \mathrm{mg}, 0.11 \mathrm{mmol}) .{ }^{1} \mathbf{H}$ NMR $\left(500 \mathrm{MHz}, \mathrm{CDCl}_{3}\right) \delta$<smiles>Brc1ccc(CNCc2ccc(Br)cc2)cc1</smiles>
$7.45(\mathrm{~d}, J=8.3 \mathrm{~Hz}, 3 \mathrm{H}), 7.21$ (d, $J=8.2 \mathrm{~Hz}, 3 \mathrm{H}), 3.73(\mathrm{~s}, 3 \mathrm{H}), 1.63$ $(\mathrm{s}, 1 \mathrm{H}) .{ }^{13} \mathbf{C}$ NMR $\left(126 \mathrm{MHz}, \mathrm{CDCl}_{3}\right) \delta 139.2,131.6,129.9,120.9$, Br 52.5 .

Dimethyl 4,4' -(azanediylbis(methylene))dibenzoate(3i) ${ }^{16}$ : Yield $=65 \%(26 \mathrm{mg}, 0.08 \mathrm{mmol})$.<smiles>COC(=O)c1ccc(CNCc2ccc(C(C)=O)cc2)cc1</smiles>
${ }^{1} \mathbf{H}$ NMR $\left(400 \mathrm{MHz}, \mathrm{CDCl}_{3}\right): \delta 7.98(\mathrm{~d}, J=8.1 \mathrm{~Hz}, 4 \mathrm{H})$, $7.39(\mathrm{~d}, J=8.1 \mathrm{~Hz}, 4 \mathrm{H}), 3.89(\mathrm{~s}, 6 \mathrm{H}), 3.83(\mathrm{~s}, 4 \mathrm{H}) .{ }^{\mathbf{1 3}} \mathbf{C}$ NMR $\left(101 \mathrm{MHz}, \mathrm{CDCl}_{3}\right) \delta 167.1,145.5,129.9,129.1$, $128.1,77.5,77.2,76.8,52.9,52.2$. 
N-benzyl-1-(4-methoxyphenyl)methanamine(4a) ${ }^{7}$ : Yield $=66 \%(37 \mathrm{mg}, 0.17 \mathrm{mmol}) .{ }^{1} \mathbf{H}$ NMR<smiles>COc1ccc(CNCc2ccccc2)cc1</smiles>
$\left(500 \mathrm{MHz}, \mathrm{CDCl}_{3}\right) \delta 7.37(\mathrm{~d}, J=3.9 \mathrm{~Hz}, 5 \mathrm{H}), 7.30(\mathrm{~s}, 1 \mathrm{H}), 7.28(\mathrm{~s}$, $1 \mathrm{H}), 6.91(\mathrm{~d}, J=8.6 \mathrm{~Hz}, 2 \mathrm{H}), 3.83(\mathrm{~s}, 2 \mathrm{H}), 3.83(\mathrm{~s}, 3 \mathrm{H}), 3.79(\mathrm{~s}, 2 \mathrm{H})$, $2.98(\mathrm{~s}, 1 \mathrm{H}) .{ }^{13} \mathbf{C}$ NMR $\left(101 \mathrm{MHz}, \mathrm{CDCl}_{3}\right) \delta 158.7,140.5,132.5$, $129.4,128.5,128.3,127.0,113.9,55.4,53.1,52.6$.

N-benzyl-1-(4-chlorophenyl)methanamine(4b) ${ }^{17}:$ Yield = 52\% (31 mg, $\left.0.13 \mathrm{mmol}\right){ }^{1} \mathbf{H}$ NMR (500<smiles>Clc1ccc(CNCc2ccccc2)cc1</smiles>
$\left.\mathrm{MHz}, \mathrm{CDCl}_{3}\right): \delta 7.29-7.17(\mathrm{~m}, 9 \mathrm{H}), 3.70(\mathrm{t}, J=10.6 \mathrm{~Hz}, 4 \mathrm{H}), 1.62(\mathrm{~s}$, 1H). ${ }^{13} \mathbf{C ~ N M R ~}\left(126 \mathrm{MHz}, \mathrm{CDCl}_{3}\right): \delta 140 ., 138.8,132.6,129.4,129.4$, $128.5,128.4,128.4,128.0,127.0,53.0,52.3$

N-benzyl-1-(pyridin-2-yl)methanamine(4c) ${ }^{18}$ : Yield = 60\% (30 mg, $\left.0.15 \mathrm{mmol}\right){ }^{1} \mathbf{H}$ NMR $(500 \mathrm{MHz}$,<smiles>c1ccc(CNCc2ccccn2)cc1</smiles>
$\left.\mathrm{CDCl}_{3}\right): \delta 8.56(\mathrm{~d}, J=4.6 \mathrm{~Hz}, 1 \mathrm{H}), 7.60-7.69(\mathrm{~m}, 1 \mathrm{H}), 7.38-7.19(\mathrm{~m}, 6 \mathrm{H})$, $7.16(\mathrm{dd}, J=6.9,5.2 \mathrm{~Hz}, 1 \mathrm{H}), 3.93(\mathrm{~s}, 2 \mathrm{H}), 3.85(\mathrm{~s}, 2 \mathrm{H}), 2.07(\mathrm{~s}, 2 \mathrm{H}) \cdot{ }^{13} \mathbf{C}$ NMR $\left(126 \mathrm{MHz}, \mathrm{CDCl}_{3}\right): \delta 159.7,149.3,140.1,136.4,128.4,128.2,127$, $122.3,121.9,54.5,53.5$.

N-benzylcyclohexanamine(4d) $)^{7}$ : Yield $=60 \%(57 \mathrm{mg}, 0.29 \mathrm{mmol}) .{ }^{1} \mathbf{H}$ NMR $\left(400 \mathrm{MHz}, \mathrm{CDCl}_{3}\right) \delta$<smiles>c1ccc(CNC2CCCCC2)cc1</smiles>
$7.30(\mathrm{~d}, J=4.4 \mathrm{~Hz}, 4 \mathrm{H}), 7.26-7.20(\mathrm{~m}, 1 \mathrm{H}), 3.80(\mathrm{~s}, 2 \mathrm{H}), 2.47(\mathrm{tt}, J=10.1,3.7$ $\mathrm{Hz}, 1 \mathrm{H}), 1.94-1.86(\mathrm{~m}, 2 \mathrm{H}), 1.77-1.68(\mathrm{~m}, 2 \mathrm{H}), 1.55-1.64(\mathrm{~m}, 1 \mathrm{H}), 1.22-1.31$ $(\mathrm{m}, 2 \mathrm{H}), 1.18-1.06(\mathrm{~m}, 3 \mathrm{H}) .{ }^{13} \mathbf{C}$ NMR $\left(101 \mathrm{MHz}, \mathrm{CDCl}_{3}\right) \delta 140.8,128.2,127.9$, 126.6, 56.0, 50.8, 33.4, 26.0, 24.8 .

N-(4-methylbenzyl)butan-1-amine(4e) ${ }^{14}$ : Yield $=54 \%(24 \mathrm{mg}, 0.13 \mathrm{mmol}) .{ }^{1} \mathbf{H}$ NMR $(400 \mathrm{MHz}$, $\left.\mathrm{CDCl}_{3}\right) \delta 7.21(\mathrm{~d}, J=7.9 \mathrm{~Hz}, 2 \mathrm{H}), 7.13(\mathrm{~d}, J=7.9 \mathrm{~Hz}, 2 \mathrm{H}), 3.75(\mathrm{~s}, 2 \mathrm{H})$, $2.65-2.58(\mathrm{~m}, 2 \mathrm{H}), 2.34(\mathrm{~s}, 3 \mathrm{H}), 1.52-1.47(\mathrm{~m}, 2 \mathrm{H}), 1.39-1.31(\mathrm{~m}, 2 \mathrm{H})$, $1.26(\mathrm{~s}, 1 \mathrm{H}), 0.91(\mathrm{t}, J=7.3 \mathrm{~Hz}, 3 \mathrm{H}) .{ }^{13} \mathbf{C} \mathbf{N M R}\left(101 \mathrm{MHz}, \mathrm{CDCl}_{3}\right) \delta 137.6$, $136.5,129.2,128.2,77.5,77.2,76.8,53.9,49.3,32.4,21.2,20.6,14.16$.

N-(4-methoxybenzyl)-1-(p-tolyl)methanamine(4f) ${ }^{19}$ : Yield = 52\% (30 mg, $\left.0.12 \mathrm{mmol}\right) .{ }^{1} \mathbf{H}$ NMR<smiles>COc1ccc(CNCc2ccc(C)cc2)cc1</smiles>
$\left(500 \mathrm{MHz}, \mathrm{CDCl}_{3}\right): \delta 7.25(\mathrm{dd}, J=15.2,8.2 \mathrm{~Hz}, 4 \mathrm{H}), 7.16(\mathrm{~s}, 2 \mathrm{H})$, $6.88(\mathrm{~d}, J=8.5 \mathrm{~Hz}, 2 \mathrm{H}), 3.81(\mathrm{~s}, 3 \mathrm{H}), 3.76(\mathrm{~d}, J=10.3 \mathrm{~Hz}, 4 \mathrm{H}), 2.36$ (s, 3H), 1.73 (s, 2H) ${ }^{13} \mathbf{C}$ NMR $\left(126 \mathrm{MHz}, \mathrm{CDCl}_{3}\right): \delta$ 158.6, 137.3, $136.4,132.5,129.3,129.0,128.0,113.7,55.2,52.7,52.4,21.0$

N-(4-methoxybenzyl)-1-(m-tolyl) methanamine(4g) ${ }^{19}$ : Yield $=50 \%$ (30 mg, $\left.0.12 \mathrm{mmol}\right) .{ }^{\mathbf{1}} \mathbf{H}$ NMR<smiles>COc1ccc(CNCc2cccc(C)c2)cc1</smiles>
$\left(500 \mathrm{MHz}, \mathrm{CDCl}_{3}\right) \delta 7.31-7.28(\mathrm{~m}, 2 \mathrm{H}), 7.26(\mathrm{q}, J=7.5 \mathrm{~Hz}, 1 \mathrm{H}), 7.19$ $(\mathrm{d}, J=0.6 \mathrm{~Hz}, 1 \mathrm{H}), 7.16(\mathrm{~d}, J=8.6 \mathrm{~Hz}, 1 \mathrm{H}), 7.11(\mathrm{~d}, J=0.5 \mathrm{~Hz}, 1 \mathrm{H})$, $6.91(\mathrm{~d}, J=8.7 \mathrm{~Hz}, 2 \mathrm{H}), 3.84(\mathrm{~s}, 3 \mathrm{H}), 3.80(\mathrm{~s}, 2 \mathrm{H}), 3.79(\mathrm{~s}, 2 \mathrm{H}), 2.39$ $(\mathrm{s}, 3 \mathrm{H}) .{ }^{13} \mathbf{C}$ NMR $\left(126 \mathrm{MHz}, \mathrm{CDCl}_{3}\right): \delta 158.6,140.2,137.9,129.3$, $128.9,128.2,127.6,125.1,113.7,55.2,53.0,52.6,21.3$.

N-(4-bromobenzyl)-1-(4-methoxyphenyl)methanamine(4h) ${ }^{17}$ : Yield $=65 \%$ (50 mg, $\left.0.16 \mathrm{mmol}\right) .{ }^{\mathbf{1}} \mathbf{H}$<smiles>COc1ccc(CNCc2ccc(Br)cc2)cc1</smiles>
NMR $\left(500 \mathrm{MHz}, \mathrm{CDCl}_{3}\right) \delta 7.45(\mathrm{~d}, J=8.2 \mathrm{~Hz}, 2 \mathrm{H}), 7.23(\mathrm{dd}, J=$ $11.6,8.4 \mathrm{~Hz}, 4 \mathrm{H}), 6.88(\mathrm{~d}, J=8.3 \mathrm{~Hz}, 2 \mathrm{H}), 3.81(\mathrm{~s}, 3 \mathrm{H}), 3.74(\mathrm{~d}$, $J=8.6 \mathrm{~Hz}, 4 \mathrm{H}), 1.70(\mathrm{~s}, 1 \mathrm{H}) .{ }^{13} \mathbf{C}$ NMR $\left(126 \mathrm{MHz}, \mathrm{CDCl}_{3}\right): \delta$ 158.7, 139.3, 131.4, 129.8, 129.3, 120.6, 113.8, 55.2, 52.4, 52.3. 
N-(4-methylbenzyl)cyclohexanamine(4i) ${ }^{19}$ : Yield = 50\% (26 mg, $\left.0.13 \mathrm{mmol}\right) .{ }^{1} \mathbf{H}$ NMR $(500 \mathrm{MHz}$,<smiles>Cc1ccc(CNC2CCCCC2)cc1</smiles>
$\left.\mathrm{CDCl}_{3}\right) \delta 7.16(\mathrm{dd}, J=37.7,7.8 \mathrm{~Hz}, 4 \mathrm{H}), 5.29(\mathrm{~s}, 1 \mathrm{H}), 3.77(\mathrm{~s}, 2 \mathrm{H}), 2.52$ $-2.45(\mathrm{~m}, 1 \mathrm{H}), 2.33(\mathrm{~s}, 3 \mathrm{H}), 1.91(\mathrm{~d}, J=10.4 \mathrm{~Hz}, 2 \mathrm{H}), 1.73(\mathrm{dd}, J=9.4$, $3.4 \mathrm{~Hz}, 2 \mathrm{H}), 1.60(\mathrm{~s}, 1 \mathrm{H}), 1.56(\mathrm{~s}, 1 \mathrm{H}), 1.29-1.09(\mathrm{~m}, 5 \mathrm{H}){ }^{13} \mathbf{C}$ NMR $(126$ $\left.\mathrm{MHz}, \mathrm{CDCl}_{3}\right): \delta 137.8,136.3,129.0,128.0,56.0,53.4,50.7,33.5,26.2$, 25.0, 21.0. 


\section{Computational Details:}

All calculations were carried out using Density Functional Theory as implemented in the Gaussian0920 quantum chemistry programs. The geometries of stationary points and transition states (TS) were optimized with B3LYP functional. ${ }^{21}$ We used double- $\zeta$ basis set with the relativistic effective core potential of Hay and Wadt (LANL2DZ) 22 for the manganese atom and 6-31G* basis set for other elements $(\mathrm{H}, \mathrm{C}, \mathrm{O}, \mathrm{S}$ and $\mathrm{N})$. The geometries were optimized without any symmetry constraints. Harmonic force constants were computed at the optimized geometries to characterize the stationary points as minima or saddle points. The zero-point vibrational corrections were determined from the harmonic vibrational frequencies to convert the total energies $\mathrm{E}^{\mathrm{e}}$ to $\mathrm{E}^{0}$. The rigid-rotor harmonicoscillator approximation was applied for evaluating the thermal and entropic contribution that were necessary to obtain the enthalpies, $\mathrm{H}_{298}$ and Gibbs free energies, $\mathrm{G}_{298}$ at $298 \mathrm{~K}$. To get accurate energies of the optimized structures were reevaluated by additional single point energy calculations of each optimized geometry using $6-311 \mathrm{G}^{*}$ triple- $\zeta$ basis $\operatorname{set}^{23}$ for light atoms $(\mathrm{H}, \mathrm{C}, \mathrm{N}, \mathrm{S}, \mathrm{O})$. The solvent correction was done using the polarized continuum model. ${ }^{24}$

\section{Cartesian Coordinates for Optimized geometries and Transition States}

\begin{tabular}{lccc} 
I & & & \\
O 1 & & & \\
Mn & 1.12569900 & 1.43150200 & 1.18600600 \\
O & -1.48387600 & 2.79658700 & 1.30161800 \\
$\mathrm{O}$ & 1.48051100 & 1.76022500 & -1.72777800 \\
$\mathrm{~N}$ & 2.14034800 & 3.13516900 & 1.60243800 \\
$\mathrm{C}$ & 3.01013300 & 3.03567400 & 2.62826600 \\
$\mathrm{C}$ & 2.01602700 & 4.32557000 & 0.97564200 \\
$\mathrm{H}$ & 1.30539000 & 4.35535900 & 0.15758900 \\
$\mathrm{C}$ & 3.64545900 & 5.34671500 & 2.40740500 \\
$\mathrm{H}$ & 4.23225900 & 6.20494800 & 2.72178100 \\
$\mathrm{C}$ & 2.74398000 & 5.44740300 & 1.34364900 \\
$\mathrm{H}$ & 2.60519200 & 6.37911100 & 0.80515200 \\
$\mathrm{C}$ & 3.07859900 & 1.67702500 & 3.25714400 \\
$\mathrm{H}$ & 4.13213900 & 1.33679300 & 3.25570700 \\
$\mathrm{H}$ & 2.80651900 & 1.77168300 & 4.32720600 \\
$\mathrm{C}$ & 2.29110000 & -0.57067100 & 3.17664900 \\
$\mathrm{H}$ & 3.21685300 & -0.65049400 & 3.76614700 \\
$\mathrm{H}$ & 1.47516000 & -0.69869100 & 3.91151500 \\
$\mathrm{C}$ & -0.45784800 & 2.24877500 & 1.20561600 \\
$\mathrm{C}$ & 1.29261100 & 1.63228800 & -0.58570700 \\
$\mathrm{C}$ & 3.77675000 & 4.12352300 & 3.05460100 \\
$\mathrm{H}$ & 4.46537600 & 3.99896300 & 3.88527800 \\
$\mathrm{~N}$ & 2.21049000 & 0.75220300 & 2.55508600 \\
$\mathrm{C}$ & 2.28120900 & -1.75774000 & 2.23037400 \\
$\mathrm{C}$ & 3.24459800 & -2.76344800 & 2.38219400 \\
$\mathrm{C}$ & 1.31717400 & -1.92537800 & 1.22142600 \\
$\mathrm{C}$ & 3.26174100 & -3.89187200 & 1.56077900 \\
$\mathrm{H}$ & 3.99975000 & -2.65438900 & 3.15732000 \\
$\mathrm{C}$ & 1.33435100 & -3.04934700 & 0.38805100 \\
$\mathrm{C}$ & 2.30456100 & -4.03748100 & 0.55744500 \\
$\mathrm{H}$ & 4.02771300 & -4.64983700 & 1.70099900 \\
$\mathrm{H}$ & 0.58537100 & -3.14195400 & -0.39305700 \\
$\mathrm{H}$ & 2.31279900 & -4.90821300 & -0.09211800 \\
$\mathrm{~S}$ & 0.07850800 & -0.66624800 & 0.89052400 \\
$\mathrm{C}$ & -1.09321800 & -0.93411100 & 2.28042700 \\
$\mathrm{H}$ & -1.91717500 & -0.23400600 & 2.12301300 \\
$\mathrm{H}$ & -1.46869900 & -1.95965300 & 2.24986500 \\
$\mathrm{H}$ & -0.62372200 & -0.72531700 & 3.24348300 \\
& & & \\
\hline
\end{tabular}

II

01

$\begin{array}{rrr}1.01209900 & 1.34549700 & 1.16437100 \\ -1.51004700 & 2.85061400 & 1.42746800 \\ 1.19570900 & 1.62607800 & -1.76618700 \\ 2.12226400 & 3.00762400 & 1.52190200 \\ 3.01774000 & 2.88289400 & 2.52353800 \\ 2.03021400 & 4.19370900 & 0.88054400 \\ 1.29697000 & 4.24418900 & 0.08361400 \\ 3.74945500 & 5.15786100 & 2.24450300 \\ 4.38768500 & 5.99118100 & 2.52330500 \\ 2.81756900 & 5.28645800 & 1.20985000 \\ 2.70363200 & 6.21623200 & 0.66237600 \\ 3.05018800 & 1.53385400 & 3.17200900 \\ 4.09112600 & 1.17059100 & 3.19830100 \\ 2.73724100 & 1.64082300 & 4.22788600 \\ 2.21663800 & -0.70435800 & 3.09835100 \\ 3.17951400 & -0.82949500 & 3.61206200 \\ 1.45024500 & -0.76530700 & 3.89144100 \\ -0.52570900 & 2.24552300 & 1.27712700 \\ 1.06598700 & 1.52859400 & -0.61194300 \\ 3.84894200 & 3.93978800 & 2.90535800 \\ 4.56584200 & 3.79088900 & 3.70678400 \\ 2.17081200 & 0.61565600 & 2.45464900 \\ 2.06083100 & -1.89634100 & 2.17170400 \\ 2.99009800 & -2.94429600 & 2.24591200 \\ 1.01125000 & -2.01761300 & 1.24312500 \\ 2.88552000 & -4.07265700 & 1.42944700 \\ 3.81921500 & -2.86149500 & 2.94350100 \\ 0.90557200 & -3.14387000 & 0.41812000 \\ 1.83992500 & -4.17594400 & 0.51192100 \\ 3.62652700 & -4.86339000 & 1.50717400 \\ 0.09045800 & -3.20303900 & -0.29723800 \\ 1.75238900 & -5.04784800 & -0.13008500 \\ -0.17768900 & -0.69375100 & 0.98903600 \\ -1.24210000 & -0.85959800 & 2.47747800 \\ -2.03842300 & -0.11984700 & 2.36619300 \\ -1.67223100 & -1.86332000 & 2.50669000 \\ -0.68394800 & -0.65318700 & 3.39233700 \\ 3.63251200 & 0.14978900 & 0.88359900 \\ 4.47328600 & -0.20026700 & 0.39632800 \\ 4.56377800 & 0.32400700 & -0.47489300 \\ 4.28669100 & -1.17351800 & 0.15062900 \\ 5.82921900 & -0.06777900 & 1.32403800 \\ 5.59485400 & -0.70901800 & 2.33217800 \\ 6.73446600 & -0.53589900 & 0.66795200 \\ \end{array}$

$\begin{array}{lll}1.01209900 & 1.34549700 & 1.16437100\end{array}$

$-1.51004700-285061400-1.42746800$

$\begin{array}{lllll}\mathrm{O} & 1.19570900 & 1.62607800 & -1.76618700\end{array}$

$\mathrm{N} \quad 2.12226400 \quad 3.00762400 \quad 1.52190200$

$\begin{array}{llll}\mathrm{C} & 3.01774000 & 2.88289400 & 2.52353800\end{array}$

C $\quad 2.03021400 \quad 4.19370900 \quad 0.88054400$

$\begin{array}{llll}\mathrm{H} & 1.29697000 & 4.24418900 & 0.08361400\end{array}$

$\begin{array}{llll}\mathrm{C} & 3.74945500 & 5.15786100 & 2.24450300\end{array}$

$\begin{array}{llll}\mathrm{H} & 4.38768500 & 5.99118100 & 2.52330500\end{array}$

$\begin{array}{llll}\mathrm{C} & 2.81756900 & 5.28645800 & 1.20985000\end{array}$

$\begin{array}{llll}\mathrm{H} & 2.70363200 & 6.21623200 & 0.66237600\end{array}$

$\begin{array}{llll}\mathrm{C} & 3.05018800 & 1.53385400 & 3.17200900\end{array}$

$\begin{array}{llll}\mathrm{H} & 4.09112600 & 1.17059100 & 3.19830100\end{array}$

$\begin{array}{llll}\mathrm{H} & 2.73724100 & 1.64082300 & 4.22788600\end{array}$

$\begin{array}{llll}\mathrm{C} & 2.21663800 & -0.70435800 & 3.09835100 \\ \mathrm{H} & 3.17951400 & -0.82949500 & 3.61206200\end{array}$

$\begin{array}{llll}\mathrm{H} & 3.17951400 & -0.82949500 & 3.61206200 \\ \mathrm{H} & 1.45024500 & -0.76530700 & 3.89144100\end{array}$

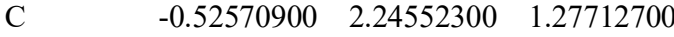

$\begin{array}{lllll}\mathrm{C} & 1.06598700 & 1.52859400 & -0.61194300\end{array}$

$\begin{array}{llll}\mathrm{C} & 3.84894200 & 3.93978800 & 2.90535800\end{array}$

$\begin{array}{llll}\mathrm{H} & 4.56584200 & 3.79088900 & 3.70678400\end{array}$

$\begin{array}{llll}\mathrm{N} & 2.17081200 & 0.61565600 & 2.45464900\end{array}$

$\begin{array}{llll}\mathrm{C} & 2.06083100 & -1.89634100 & 2.17170400\end{array}$

$\begin{array}{llll}\text { C } & 2.99009800 & -2.94429600 & 2.24591200\end{array}$

$\begin{array}{llll}\mathrm{C} & 1.01125000 & -2.01761300 & 1.24312500\end{array}$

$\begin{array}{llll}\mathrm{C} & 2.88552000 & -4.07265700 & 1.42944700\end{array}$

$\begin{array}{llll}\mathrm{H} & 3.81921500 & -2.86149500 & 2.94350100\end{array}$

$\begin{array}{llll}\mathrm{C} & 0.90557200 & -3.14387000 & 0.41812000\end{array}$

$\begin{array}{llll}\mathrm{C} & 1.83992500 & -4.17594400 & 0.51192100\end{array}$

$\mathrm{H} \quad 3.62652700 \quad-4.86339000 \quad 1.50717400$

$\mathrm{H} \quad 0.09045800 \quad-3.20303900 \quad-0.29723800$

$\mathrm{H} \quad \begin{array}{llll}\mathrm{H} & 1.75238900 & -5.04784800 & -0.13008500\end{array}$

$\begin{array}{llll}\mathrm{S} & -0.17768900 & -0.69375100 & 0.98903600\end{array}$

$\begin{array}{llll}\text { C } & -1.24210000 & -0.85959800 & 2.47747800\end{array}$

$\mathrm{H} \quad-2.03842300 \quad-0.11984700 \quad 2.36619300$

$\mathrm{H} \quad-1.67223100 \quad-1.86332000 \quad 2.50669000$

$\mathrm{H} \quad-\quad-0.68394800 \quad-0.65318700 \quad 3.39233700$

$\begin{array}{llll}\mathrm{H} & 3.63251200 & 0.14978900 & 0.88359900\end{array}$

$\begin{array}{llll}\mathrm{N} & 4.47328600 & -0.20026700 & 0.39632800\end{array}$

$\mathrm{H} \quad \begin{array}{llll}\mathrm{H} & 4.56377800 & 0.32400700 & -0.47489300\end{array}$

H $\quad 4.28669100 \quad-1.17351800 \quad 0.15062900$

$\begin{array}{llll}\mathrm{B} & 5.82921900 & -0.06777900 & 1.32403800 \\ \mathrm{H} & 5.59485400 & -0.70901800 & 2.33217800\end{array}$

$\begin{array}{llll}\mathrm{H} & 5.59485400 & -0.7501800 & 2.33217800 \\ \mathrm{H} & 6.73446600 & -0.53589900 & 0.66795200\end{array}$ 


\begin{tabular}{|c|c|c|c|}
\hline $\mathrm{H}$ & 5.95900000 & 1.12173000 & 1.53876800 \\
\hline III & & & \\
\hline \multicolumn{4}{|l|}{01} \\
\hline $\mathrm{Mn}$ & 1.00185900 & 1.49832700 & 1.32719600 \\
\hline $\mathrm{O}$ & -0.63321900 & 1.77458400 & 3.77842800 \\
\hline $\mathrm{O}$ & -1.10307000 & 2.78062500 & -0.30601800 \\
\hline $\mathrm{N}$ & 2.06090700 & 3.20787100 & 1.68426400 \\
\hline $\mathrm{C}$ & 3.09813500 & 3.06779900 & 2.53822800 \\
\hline $\mathrm{C}$ & 1.89242100 & 4.39737700 & 1.07571100 \\
\hline $\mathrm{H}$ & 1.04759000 & 4.47090300 & 0.40037300 \\
\hline $\mathrm{C}$ & 3.80823200 & 5.33604400 & 2.16744200 \\
\hline $\mathrm{H}$ & 4.49288900 & 6.15831900 & 2.35282900 \\
\hline $\mathrm{C}$ & 2.73549700 & 5.48236900 & 1.28907200 \\
\hline $\mathrm{H}$ & 2.55012400 & 6.41663200 & 0.76952100 \\
\hline $\mathrm{C}$ & 3.18863200 & 1.73612800 & 3.23600100 \\
\hline $\mathrm{H}$ & 4.22575300 & 1.52362000 & 3.53132100 \\
\hline $\mathrm{H}$ & 2.58809500 & 1.78074900 & 4.15549400 \\
\hline $\mathrm{C}$ & 2.40738300 & -0.57050700 & 3.17150400 \\
\hline $\mathrm{H}$ & 3.23577000 & -0.71850600 & 3.87914700 \\
\hline $\mathrm{H}$ & 1.50011100 & -0.42268000 & 3.77018500 \\
\hline $\mathrm{C}$ & 0.01626800 & 1.66164900 & 2.81685400 \\
\hline $\mathrm{C}$ & -0.28363000 & 2.26524500 & 0.34049500 \\
\hline $\mathrm{C}$ & 3.98864700 & 4.10836500 & 2.79870700 \\
\hline $\mathrm{H}$ & 4.81711700 & 3.94614700 & 3.48110600 \\
\hline $\mathrm{N}$ & 2.63211500 & 0.66687200 & 2.38781900 \\
\hline $\mathrm{C}$ & 2.30160100 & -1.81825800 & 2.31941700 \\
\hline $\mathrm{C}$ & 3.17127100 & -2.88346000 & 2.58928500 \\
\hline $\mathrm{C}$ & 1.36562800 & -1.97994300 & 1.28045300 \\
\hline $\mathrm{C}$ & 3.12062900 & -4.07412600 & 1.86553200 \\
\hline $\mathrm{H}$ & 3.90668200 & -2.77140400 & 3.38212100 \\
\hline $\mathrm{C}$ & 1.32809700 & -3.16683700 & 0.53915100 \\
\hline $\mathrm{C}$ & 2.19461300 & -4.21847800 & 0.83406100 \\
\hline $\mathrm{H}$ & 3.81188600 & -4.87857000 & 2.10005300 \\
\hline $\mathrm{H}$ & 0.61850700 & -3.25693400 & -0.27817600 \\
\hline $\mathrm{H}$ & 2.15343400 & -5.13476700 & 0.25224900 \\
\hline S & 0.21061400 & -0.68738300 & 0.78102200 \\
\hline $\mathrm{C}$ & -1.25813700 & -1.15080100 & 1.77963300 \\
\hline $\mathrm{H}$ & -2.05201800 & -0.45101500 & 1.50677100 \\
\hline $\mathrm{H}$ & -1.56308900 & -2.16930400 & 1.52911100 \\
\hline $\mathrm{H}$ & -1.05371400 & -1.06538500 & 2.84878300 \\
\hline $\mathrm{H}$ & 3.30476200 & 0.46870500 & 1.63035000 \\
\hline $\mathrm{N}$ & 2.22556700 & 1.32763300 & -0.41927200 \\
\hline $\mathrm{H}$ & 2.19677700 & 2.24845900 & -0.85799700 \\
\hline $\mathrm{H}$ & 1.75959900 & 0.72595700 & -1.10005100 \\
\hline B & 3.77074900 & 0.89818600 & -0.36647700 \\
\hline $\mathrm{H}$ & 4.36404900 & 1.67720600 & 0.37600000 \\
\hline $\mathrm{H}$ & 3.84256200 & -0.24832800 & 0.07320000 \\
\hline $\mathrm{H}$ & 4.24908700 & 0.93725600 & -1.48776200 \\
\hline
\end{tabular}

IV

01

$\begin{array}{llll}\text { Mn } & 1.13520700 & 1.57985200 & 1.32387400\end{array}$

$\begin{array}{lllll}\mathrm{O} & -0.78307500 & 1.69618100 & 3.57998500\end{array}$

$\begin{array}{lllll}\mathrm{O} & -0.88551300 & 2.67695500 & -0.53996500\end{array}$

$\begin{array}{llll}\mathrm{N} & 2.07199700 & 3.31196900 & 1.71852900\end{array}$

$\begin{array}{llll}\text { C } & 3.04060400 & 3.23449700 & 2.66051200\end{array}$

$\begin{array}{llll}\mathrm{C} & 1.94258900 & 4.45825700 & 1.02449200\end{array}$

$\begin{array}{llll}\mathrm{H} & 1.16408800 & 4.47500800 & 0.27188200\end{array}$

$\begin{array}{llll}\mathrm{C} & 3.75367100 & 5.48727300 & 2.21062500\end{array}$

$\begin{array}{llll}\mathrm{H} & 4.41333200 & 6.32876100 & 2.39989900\end{array}$

$\begin{array}{llll}\mathrm{C} & 2.75330900 & 5.56596900 & 1.24395000\end{array}$

$\begin{array}{llll}\mathrm{H} & 2.60360800 & 6.46350100 & 0.65340400\end{array}$

$\begin{array}{llll}\mathrm{C} & 3.10242000 & 1.93594000 & 3.42440900\end{array}$

$\begin{array}{llll}\mathrm{H} & 4.10483400 & 1.78016700 & 3.84206300\end{array}$
01 $\mathrm{Mn}$

O

O

$\mathrm{N}$

C

C

$\mathrm{H}$

C

$\mathrm{H}$

$\mathrm{H}$

$\mathrm{C}$
$\mathrm{H}$

$\mathrm{H}$

C

$\mathrm{H}$

$\mathrm{H}$

C

C

$\mathrm{H}$

C

C

C

C

$\mathrm{H}$

C

$\begin{array}{rrr}2.39585900 & 1.97981500 & 4.26471800 \\ 2.43507400 & -0.45125300 & 3.25414100 \\ 1.68424100 & -0.25123000 & 4.02558000 \\ 1.99084300 & -1.13295300 & 2.52432700 \\ -0.01473700 & 1.70056400 & 2.70575800 \\ -0.09563400 & 2.24015900 & 0.19018800 \\ 3.89843400 & 4.29945800 & 2.92397100 \\ 4.67384200 & 4.18782200 & 3.67535100 \\ 2.68407200 & 0.82992700 & 2.54200600 \\ 3.66165700 & -1.08865800 & 3.87869700 \\ 3.93312400 & -0.86998900 & 5.23809300 \\ 4.54794200 & -1.90144500 & 3.13894600 \\ 5.05077800 & -1.42593600 & 5.85850600 \\ 3.24372100 & -0.26300000 & 5.82094900 \\ 5.67852600 & -2.44552000 & 3.76491600 \\ 5.92921800 & -2.21691500 & 5.11692200 \\ 5.23354100 & -1.24242600 & 6.91364400 \\ 6.36622300 & -3.04798900 & 3.17866100 \\ 6.80901900 & -2.64935300 & 5.58534900 \\ 4.28374000 & -2.24507500 & 1.38910500 \\ 3.76938700 & -4.00570900 & 1.48359300 \\ 3.61350100 & -4.33638400 & 0.45291200 \\ 4.55111400 & -4.61911900 & 1.93880100 \\ 2.83722100 & -4.11988200 & 2.04326200 \\ 3.43099800 & 0.70844900 & 1.83931100 \\ 2.26240100 & 0.77942500 & -0.21600500 \\ 1.78860300 & 0.85251400 & -1.11463100 \\ 2.35512100 & -0.23005500 & -0.05970400 \\ 3.76776100 & 1.34083500 & -0.45287600 \\ 3.69675700 & 2.53551400 & -0.68093900 \\ 4.42644400 & 1.14081500 & 0.57524300 \\ 4.27350600 & 0.71794300 & -1.37039300\end{array}$

$\begin{array}{rrr}0.93439100 & 1.51968700 & 1.39424900 \\ -0.94686000 & 2.10079100 & 3.62194700 \\ -0.74554100 & 2.76148000 & -0.67925200 \\ 2.09799200 & 3.16953500 & 1.66517400 \\ 3.03316600 & 3.07186300 & 2.64016600 \\ 2.06408500 & 4.30689900 & 0.94212400 \\ 1.31145400 & 4.34957700 & 0.16434500 \\ 3.90060800 & 5.26314600 & 2.15999600 \\ 4.60042800 & 6.07106700 & 2.35152500 \\ 2.93517400 & 5.36946700 & 1.15980000 \\ 2.85397200 & 6.25975000 & 0.54448400 \\ 2.98823700 & 1.78562700 & 3.42244400 \\ 3.96056500 & 1.57509500 & 3.89451500 \\ 2.23853100 & 1.86578600 & 4.22114200 \\ 2.33383700 & -0.57634700 & 3.23432300 \\ 3.13242400 & -0.74658300 & 3.97235400 \\ 1.39333000 & -0.47876800 & 3.78868200 \\ -0.16454700 & 1.86746600 & 2.78443000 \\ -0.11937900 & 2.25802200 & 0.16585200 \\ 3.94933000 & 4.08637900 & 2.90627700 \\ 4.68723900 & 3.95065000 & 3.69159700 \\ 2.55036800 & 0.70320000 & 2.52495000 \\ 2.30777800 & -1.75012400 & 2.27948600 \\ 3.26379400 & -2.76363000 & 2.42775900 \\ 1.37002800 & -1.86218500 & 1.23341000 \\ 3.30012000 & -3.86373400 & 1.57013100 \\ 3.99261600 & -2.68623900 & 3.23153400 \\ 1.41761800 & -2.95877600 & 0.36574500 \\ 2.37463900 & -3.96085300 & 0.53252000 \\ 4.05275700 & -4.63460000 & 1.71001600 \\ 0.69868100 & -3.01759500 & -0.44603500\end{array}$




\begin{abstract}
$2.39725100 \quad-4.80712300 \quad-0.14838100$ $\begin{array}{lll}0.09857500 & -0.61809500 & 0.91908800\end{array}$ $\begin{array}{lll}-1.17636900 & -1.20629100 & 2.11157200\end{array}$ $\begin{array}{lll}-2.02553300 & -0.52645300 & 2.00784300\end{array}$ $\begin{array}{lll}-1.48456000 & -2.22346000 & 1.85668700\end{array}$ $-0.81410800 \quad-1.16647300 \quad 3.14159100$ $3.25795400 \quad 0.56218600 \quad 1.80488900$ $\begin{array}{lll}1.84586300 & 1.18385300 & 0.08314800\end{array}$
\end{abstract}

\section{VI}

01

$\begin{array}{llll}\text { Mn } & 0.67462800 & 1.60655900 & 2.08903300\end{array}$

$\begin{array}{llll}\mathrm{O} & -0.44192100 & 2.93122500 & 4.51419500\end{array}$

$\begin{array}{llll}\mathrm{O} & -1.47051200 & 2.49013300 & 0.27335800\end{array}$

$\begin{array}{llll}\mathrm{N} & 2.00918300 & 3.02353800 & 1.62028300\end{array}$

$\begin{array}{llll}\mathrm{C} & 3.19816200 & 2.92083600 & 2.26523900\end{array}$

$\begin{array}{llll}\mathrm{C} & 1.88438900 & 3.97090900 & 0.66936000\end{array}$

$\begin{array}{lllll}\mathrm{H} & 0.92233200 & 4.01782000 & 0.17372600\end{array}$

$\begin{array}{llll}\mathrm{C} & 4.14274200 & 4.72464600 & 0.98140100\end{array}$

$\begin{array}{lllll}\mathrm{H} & 4.97241100 & 5.37959800 & 0.73301300\end{array}$

$\begin{array}{lllll}\mathrm{C} & 2.91730600 & 4.83729200 & 0.32673600\end{array}$

$\begin{array}{llll}\mathrm{H} & 2.75505700 & 5.58023400 & -0.44742800\end{array}$

$\begin{array}{llll}\mathrm{C} & 3.25004900 & 1.87348900 & 3.34967000\end{array}$

$\begin{array}{llll}\mathrm{H} & 4.28652900 & 1.57239500 & 3.55209000\end{array}$

$\begin{array}{llll}\mathrm{H} & 2.83736600 & 2.29372200 & 4.27735000\end{array}$

$\begin{array}{llll}\mathrm{C} & 2.14950200 & -0.24226500 & 4.04813000\end{array}$

$\begin{array}{llll}\mathrm{H} & 1.56339600 & 0.26563600 & 4.82042600\end{array}$

$\mathrm{H} \quad \begin{array}{llll}\mathrm{H} & 1.50938200 & -1.01776700 & 3.61388100\end{array}$

$\begin{array}{llll}\mathrm{C} & 0.05193200 & 2.43186900 & 3.58195800\end{array}$

$\begin{array}{llll}\mathrm{C} & -0.64637000 & 2.15265200 & 1.02366400\end{array}$

$\begin{array}{llll}\mathrm{C} & 4.27970500 & 3.74354900 & 1.96336300\end{array}$

$\begin{array}{llll}\mathrm{H} & 5.21640900 & 3.61188200 & 2.49679200\end{array}$

$\begin{array}{llll}\mathrm{N} & 2.39023800 & 0.73939300 & 2.96557500\end{array}$

$\begin{array}{lllll}\mathrm{C} & & 3.38523600 & -0.86112400 & 4.68513300\end{array}$

$\begin{array}{llll}\mathrm{C} & 3.67238400 & -0.56594300 & 6.02470800\end{array}$

$\begin{array}{llll}\mathrm{C} & 4.25297500 & -1.73473100 & 3.99285100\end{array}$

$\begin{array}{lllll}\mathrm{C} & 4.78531100 & -1.10620300 & 6.67097800\end{array}$

$\begin{array}{llll}\mathrm{H} & 3.00673800 & 0.09884700 & 6.57062100\end{array}$

$\begin{array}{lllll}\text { C } & 5.37331300 & -2.26840000 & 4.64240200\end{array}$

$\begin{array}{llll}\mathrm{C} & 5.64149400 & -1.95944800 & 5.97693800\end{array}$

$\mathrm{H} \quad 4.98092100 \quad-0.85752000 \quad 7.71035600$

$\mathrm{H} \quad 6.03444200 \quad-2.92897900 \quad 4.08958700$

$\mathrm{H} \quad 6.51341500 \quad-2.38355500 \quad 6.46706300$

$\begin{array}{llll}\mathrm{S} & 4.00622600 & -2.14310700 & 2.25404100\end{array}$

$\begin{array}{llll}\mathrm{C} & 2.83159200 & -3.54942800 & 2.37168300\end{array}$

$\begin{array}{llll}\mathrm{H} & 2.63931200 & -3.87273500 & 1.34516500\end{array}$

$\mathrm{H} \quad 3.27402100 \quad-4.37463200 \quad 2.93492100$

$\mathrm{H} \quad 1.88672000 \quad-3.24812000 \quad 2.83070000$

$\begin{array}{llll}\mathrm{H} & 2.81555300 & 0.23979100 & 2.18398400\end{array}$

$\begin{array}{llll}\mathrm{H} & 1.02997300 & 0.67086500 & 0.78885800\end{array}$

VII

01

$\begin{array}{lrrr}\mathrm{Mn} & 0.56025700 & 1.66568300 & 2.31298000 \\ \mathrm{O} & -0.18519600 & 3.41835300 & 4.60827800 \\ \mathrm{O} & -1.53859600 & 2.62768600 & 0.48810000 \\ \mathrm{~N} & 2.01038200 & 2.90562600 & 1.57714100 \\ \mathrm{C} & 3.23055000 & 2.80270100 & 2.15320700 \\ \mathrm{C} & 1.88225300 & 3.74276400 & 0.52817300 \\ \mathrm{H} & 0.89618000 & 3.79562000 & 0.08331900 \\ \mathrm{C} & 4.19563800 & 4.37887900 & 0.61144300 \\ \mathrm{H} & 5.04406300 & 4.94400200 & 0.23700700 \\ \mathrm{C} & 2.93736900 & 4.49463900 & 0.02248000 \\ \mathrm{H} & 2.76641800 & 5.15128900 & -0.82451100 \\ \mathrm{C} & 3.29838300 & 1.88982600 & 3.34813700\end{array}$

$\begin{array}{lll}4.32707100 & 1.54112100 & 3.51265900\end{array}$

$\begin{array}{lll}2.99275300 & 2.44748700 & 4.24478200\end{array}$

$\begin{array}{lll}2.18493000 & -0.04596000 & 4.39195100\end{array}$

$\begin{array}{lll}1.69860600 & 0.57843200 & 5.14849100\end{array}$

$\begin{array}{lll}1.47979200 & -0.83703000 & 4.13011400\end{array}$

$\begin{array}{lll}0.14854100 & 2.72293700 & 3.73312400\end{array}$

$\begin{array}{lll}-0.72695800 & 2.26923800 & 1.24372700\end{array}$

$\begin{array}{llll}4.33870400 & 3.51101700 & 1.69239400\end{array}$

$\begin{array}{lll}5.29902200 & 3.37880800 & 2.18204700\end{array}$

$\begin{array}{llll}2.33934900 & 0.78588500 & 3.17868600\end{array}$

$\begin{array}{lll}3.46175900 & -0.63539300 & 4.97457900\end{array}$

$\begin{array}{lll}3.91021800 & -0.17943200 & 6.22192900\end{array}$

$\begin{array}{lll}4.21357300 & -1.63926300 & 4.32438500\end{array}$

$\begin{array}{lll}5.06778800 & -0.68515700 & 6.81555300\end{array}$

$\begin{array}{llll}3.33622000 & 0.58799300 & 6.73649200\end{array}$

$\begin{array}{lll}5.37923200 & -2.13936500 & 4.91900000\end{array}$

$\begin{array}{llll}5.80820600 & -1.66799100 & 6.16074400\end{array}$

$\begin{array}{lll}5.38861100 & -0.30879400 & 7.78308600\end{array}$

$\begin{array}{lll}5.94796300 & -2.90292200 & 4.39678800\end{array}$

$\begin{array}{lll}6.71355700 & -2.06730300 & 6.60951000\end{array}$

$\begin{array}{lll}3.75931100 & -2.26761600 & 2.69632400\end{array}$

$\begin{array}{lll}2.53811000 & -3.56690000 & 3.13334900\end{array}$

$2.24102000 \quad-4.03537600 \quad 2.19111500$

$\begin{array}{lll}2.99160500 & -4.31904700 & 3.78360400\end{array}$

$\begin{array}{lll}1.65161100 & -3.14377900 & 3.61233400\end{array}$

$\begin{array}{lll}2.64649200 & 0.18016000 & 2.41773500\end{array}$

$\begin{array}{lll}0.88059100 & 0.74850300 & 1.01208400\end{array}$

$\begin{array}{lll}-0.54044900 & 0.20424000 & 2.89373100\end{array}$

$\begin{array}{lll}-1.22415500 & -0.69436300 & 3.19213600\end{array}$

$\begin{array}{lll}-2.06182700 & -1.80805000 & 3.49756100\end{array}$

$\begin{array}{lll}-2.23057900 & -2.23162700 & 4.83060200\end{array}$

$\begin{array}{lll}-2.72648700 & -2.49100500 & 2.45913100\end{array}$

$\begin{array}{lll}-3.04872800 & -3.32183600 & 5.11376800\end{array}$

$\begin{array}{lll}-1.72381600 & -1.69837400 & 5.62888700\end{array}$

$\begin{array}{lll}-3.54390000 & -3.57749200 & 2.75751200\end{array}$

$\begin{array}{lll}-2.59528600 & -2.15874700 & 1.43432600\end{array}$

$\begin{array}{lll}-3.70660600 & -3.99641300 & 4.08131100\end{array}$

$\begin{array}{lll}-3.17679200 & -3.64355700 & 6.14341600\end{array}$

$\begin{array}{llll}-4.05624000 & -4.09906100 & 1.95410600\end{array}$

$\begin{array}{lll}-4.34608900 & -4.84484600 & 4.30791200\end{array}$

VIII

01

$\mathrm{Mn}$

$\mathrm{O}$

$\mathrm{O}$

$\mathrm{N}$

C

C

$\mathrm{H}$

$\mathrm{C}$

$\mathrm{H}$

$\mathrm{C}$

$\mathrm{H}$

$\mathrm{C}$

$\mathrm{C}$

$\mathrm{C}$

$\mathrm{H}$

$\mathrm{H}$

C

C

$\mathrm{H}$

N

C

$\begin{array}{rrr}0.76461400 & 1.59067300 & 1.51701700 \\ -0.68860500 & 1.97595000 & 4.08028000 \\ -1.34873800 & 2.88466600 & -0.07804900 \\ 1.89758000 & 3.27753600 & 1.74760700 \\ 3.00408000 & 3.13412400 & 2.51106500 \\ 1.69977100 & 4.45812400 & 1.13170300 \\ 0.80986900 & 4.52991200 & 0.51816600 \\ 3.71527100 & 5.38481800 & 2.04420200 \\ 4.42425700 & 6.19946100 & 2.15862600 \\ 2.57384300 & 5.53289200 & 1.25786400 \\ 2.35970700 & 6.46112000 & 0.73830600 \\ 3.14636700 & 1.80013400 & 3.19926600 \\ 4.20528000 & 1.58258300 & 3.40846200 \\ 2.62503100 & 1.83447200 & 4.16632200 \\ 2.45442900 & -0.54951300 & 3.07070500 \\ 3.37975600 & -0.72104100 & 3.64081600 \\ 1.63579200 & -0.50612900 & 3.80018700 \\ -0.09687100 & 1.81939200 & 3.08660000 \\ -0.53604000 & 2.36374300 & 0.57352000 \\ 3.93252100 & 4.16114100 & 2.67402900 \\ 4.81355100 & 3.99558300 & 3.28691400 \\ 2.51007200 & 0.75676400 & 2.38552600 \\ 2.27849700 & -1.70585200 & 2.10738300\end{array}$




$\begin{array}{lrrr}\mathrm{C} & 3.24376900 & -2.72146500 & 2.09878600 \\ \mathrm{C} & 1.19231500 & -1.81667200 & 1.21651400 \\ \mathrm{C} & 3.14814200 & -3.81702300 & 1.24033800 \\ \mathrm{H} & 4.08899700 & -2.64759100 & 2.77917400 \\ \mathrm{C} & 1.10653300 & -2.90651100 & 0.34311700 \\ \mathrm{C} & 2.07578700 & -3.90994600 & 0.35555400 \\ \mathrm{H} & 3.91463800 & -4.58671200 & 1.25848600 \\ \mathrm{H} & 0.27483300 & -2.95838300 & -0.35303600 \\ \mathrm{H} & 1.99458700 & -4.75049900 & -0.32770800 \\ \mathrm{~S} & -0.11837900 & -0.58124100 & 1.09474700 \\ \mathrm{C} & -1.22443900 & -1.17042300 & 2.43852600 \\ \mathrm{H} & -2.08966700 & -0.50282900 & 2.44135200 \\ \mathrm{H} & -1.54887700 & -2.19187600 & 2.22603000 \\ \mathrm{H} & -0.73510500 & -1.12282200 & 3.41379500 \\ \mathrm{H} & 2.97543600 & 0.67551200 & 1.46696500 \\ \mathrm{~N} & 1.89474900 & 1.23413600 & -0.11677800 \\ \mathrm{C} & 1.65489800 & 1.39249800 & -1.35182000 \\ \mathrm{H} & 0.68654300 & 1.77402500 & -1.73943900 \\ \mathrm{C} & 2.62123100 & 1.09542900 & -2.45130600 \\ \mathrm{C} & 2.24239800 & 1.31328500 & -3.78371400 \\ \mathrm{C} & 3.90945400 & 0.60080800 & -2.19307600 \\ \mathrm{C} & 3.12291100 & 1.04658400 & -4.83358200 \\ \mathrm{H} & 1.24480200 & 1.69611100 & -3.99273200 \\ \mathrm{C} & 4.79053600 & 0.33341900 & -3.23696100 \\ \mathrm{H} & 4.19361100 & 0.43392000 & -1.15806800 \\ \mathrm{C} & 4.40140900 & 0.55536300 & -4.56339300 \\ \mathrm{H} & 2.81183000 & 1.22171600 & -5.86075800 \\ \mathrm{H} & 5.78564000 & -0.04978500 & -3.02175300 \\ \mathrm{H} & 5.09063600 & 0.34631600 & -5.37784400\end{array}$

$\begin{array}{lrrr}\text { NH3-BH3 } & & & \\ 0 ~ 1 & & & \\ \text { B } & -2.50000100 & -0.15254200 & -0.09143800 \\ \text { H } & -1.32750000 & -0.15254200 & -0.39533400 \\ \text { H } & -3.08625100 & 0.86287400 & -0.39533700 \\ \text { H } & -3.08625100 & -1.16795900 & -0.39533700 \\ \text { N } & -2.49999900 & -0.15254200 & 1.57679500 \\ \text { H } & -2.02443700 & 0.67115600 & 1.94688400 \\ \text { H } & -3.45112500 & -0.15254300 & 1.94688200 \\ \text { H } & -2.02443700 & -0.97624000 & 1.94688400\end{array}$

\section{NH2-BH2}

$\begin{array}{llll}01 & & & \\ \text { B } & -2.70051200 & 1.07979800 & 0.04550200 \\ \text { H } & -3.26735700 & 2.13253900 & -0.01191800 \\ \text { H } & -3.27855900 & 0.03968300 & -0.08458400 \\ \text { N } & -1.32651000 & 1.06453000 & 0.27044800 \\ \text { H } & -0.77299300 & 1.90241100 & 0.38933100 \\ \text { H } & -0.78239600 & 0.21430500 & 0.33061300\end{array}$

\section{PhCN}

01
C
C
C
C
C
C
H
H
H
H
H
C
N

\section{Ph-CH-NH}

$\begin{array}{lrrr}01 & & & \\ \mathrm{C} & -1.00182600 & 0.03449300 & -0.03357900 \\ \mathrm{C} & 0.39069100 & 0.10599600 & 0.00812600 \\ \mathrm{C} & 1.03947200 & 1.34695000 & 0.06789100 \\ \mathrm{C} & 0.27098100 & 2.52267600 & 0.08543300 \\ \mathrm{C} & -1.11732000 & 2.45093200 & 0.04381900 \\ \mathrm{C} & -1.75750600 & 1.20746100 & -0.01576200 \\ \mathrm{H} & -1.49490800 & -0.93270000 & -0.07980500 \\ \mathrm{H} & 0.98269600 & -0.80696700 & -0.00564300 \\ \mathrm{H} & 0.78537900 & 3.47720000 & 0.13184000 \\ \mathrm{H} & -1.70673600 & 3.36392700 & 0.05762000 \\ \mathrm{H} & -2.84263800 & 1.15567300 & -0.04816400 \\ \mathrm{~N} & 3.15925500 & 2.49832600 & 0.16636700 \\ \mathrm{C} & 2.51269400 & 1.39731600 & 0.11120200 \\ \mathrm{H} & 4.16722700 & 2.31991900 & 0.18890200 \\ \mathrm{H} & 3.00199000 & 0.41127400 & 0.09203100\end{array}$

TS-I

$\begin{array}{lrrr}0 ~ 1 & & & \\ \mathrm{Mn} & 0.73376100 & 1.50902600 & 1.43636900 \\ \mathrm{O} & -0.99191600 & 2.17766800 & 3.70314100 \\ \mathrm{O} & -1.17218500 & 2.66986700 & -0.52605800 \\ \mathrm{~N} & 1.88352900 & 3.17686800 & 1.61730700 \\ \mathrm{C} & 2.93749200 & 3.04359100 & 2.45522500 \\ \mathrm{C} & 1.72491900 & 4.34555100 & 0.95859000 \\ \mathrm{H} & 0.86826400 & 4.41028700 & 0.29841400 \\ \mathrm{C} & 3.69123500 & 5.27338300 & 1.96408300 \\ \mathrm{H} & 4.40301500 & 6.08350000 & 2.09189000 \\ \mathrm{C} & 2.59741700 & 5.41391400 & 1.10707700 \\ \mathrm{H} & 2.42401400 & 6.33040300 & 0.55317000 \\ \mathrm{C} & 3.02917300 & 1.72569900 & 3.16913000 \\ \mathrm{H} & 4.07963100 & 1.45091000 & 3.31964800 \\ \mathrm{H} & 2.53972400 & 1.81086300 & 4.15233800 \\ \mathrm{C} & 2.31329800 & -0.59794600 & 3.05390500 \\ \mathrm{H} & 3.25332200 & -0.72803100 & 3.60265000 \\ \mathrm{H} & 1.50103200 & -0.59400900 & 3.79569200 \\ \mathrm{C} & -0.33002100 & 1.90612800 & 2.78714700 \\ \mathrm{C} & -0.43228400 & 2.21663200 & 0.24344100 \\ \mathrm{C} & 3.86332800 & 4.07114400 & 2.64013900 \\ \mathrm{H} & 4.71383700 & 3.90799100 & 3.29275900 \\ \mathrm{~N} & 2.33784000 & 0.70655400 & 2.36202100 \\ \mathrm{C} & 2.17132500 & -1.77231800 & 2.10660700 \\ \mathrm{C} & 3.15578200 & -2.76885200 & 2.10877100 \\ \mathrm{C} & 1.09306500 & -1.91991200 & 1.21501100 \\ \mathrm{C} & 3.06908300 & -3.88131200 & 1.27160400 \\ \mathrm{H} & 4.01623700 & -2.65135000 & 2.76016400 \\ \mathrm{C} & 1.00974200 & -3.02632200 & 0.36082600 \\ \mathrm{C} & 1.99251600 & -4.01502900 & 0.39542400 \\ \mathrm{H} & 3.85309800 & -4.63267000 & 1.29400300 \\ \mathrm{H} & 0.17621900 & -3.10337800 & -0.33120000 \\ \mathrm{H} & 1.92235700 & -4.87275600 & -0.26729800 \\ \mathrm{~S} & -0.19772400 & -0.67632600 & 1.04958500 \\ \mathrm{C} & -1.32335900 & -1.13998100 & 2.42575900 \\ \mathrm{H} & -2.17286500 & -0.45426000 & 2.38611600 \\ \mathrm{H} & -1.66791900 & -2.16407900 & 2.26793000 \\ \mathrm{H} & -0.82638500 & -1.05044100 & 3.39340900 \\ \mathrm{H} & 2.93032100 & 0.60217700 & 1.41175300 \\ \mathrm{~N} & 3.78992300 & 0.52630900 & 0.10772200 \\ \mathrm{H} & 3.77086800 & 1.26213500 & -0.59883200 \\ \mathrm{H} & 3.67139500 & -0.34797600 & -0.40516200 \\ \mathrm{~B} & 5.21729000 & 0.53406700 & 0.84639900 \\ \mathrm{H} & 5.40149400 & 1.67659700 & 1.26859700 \\ \mathrm{H} & 5.14819300 & -0.25530000 & 1.79650700\end{array}$


TS-IIA

01

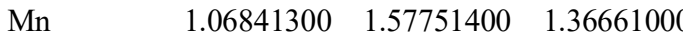

$\begin{array}{lllll}\mathrm{O} & -0.72971500 & 1.87177300 & 3.65670200\end{array}$

$\begin{array}{llll}\mathrm{O} & -0.98664400 & 2.89128000 & -0.36299300\end{array}$

$\begin{array}{llll}\mathrm{N} & 2.10826000 & 3.27616700 & 1.78857300\end{array}$

$\begin{array}{llll}\mathrm{C} & 3.09741400 & 3.12972100 & 2.69898500\end{array}$

$\begin{array}{llll}\mathrm{C} & 1.97828900 & 4.46564300 & 1.17296000\end{array}$

$\begin{array}{llll}\mathrm{H} & 1.17422000 & 4.53950100 & 0.45082900\end{array}$

$\begin{array}{llll}\mathrm{C} & 3.84418600 & 5.39069000 & 2.36181300\end{array}$

$\begin{array}{lllll}\mathrm{H} & 4.52450900 & 6.20794700 & 2.58206900\end{array}$

$\begin{array}{llll}\mathrm{C} & 2.81812500 & 5.54460200 & 1.43190200\end{array}$

$\begin{array}{llll}\mathrm{H} & 2.66715500 & 6.48014800 & 0.90363800\end{array}$

$\begin{array}{lllll}\mathrm{C} & 3.14625100 & 1.79111900 & 3.39192200\end{array}$

$\begin{array}{llll}\mathrm{H} & 4.15740000 & 1.58377300 & 3.77184800\end{array}$

$\begin{array}{llll}\mathrm{H} & 2.47224000 & 1.81675000 & 4.25868300\end{array}$

$\begin{array}{llll}\mathrm{C} & 2.39994500 & -0.53363400 & 3.23579400\end{array}$

$\begin{array}{llll}\mathrm{H} & 3.18345500 & -0.69311600 & 3.99079000\end{array}$

$\mathrm{H} \quad \begin{array}{llll}\mathrm{H} & 1.45618900 & -0.39744200 & 3.77706600\end{array}$

$\begin{array}{llll}\text { C } & -0.01387000 & 1.74917800 & 2.73966700\end{array}$

$\begin{array}{llll}\mathrm{C} & -0.12510900 & 2.34530300 & 0.19864600\end{array}$

$\begin{array}{llll}\mathrm{C} & 3.98434400 & 4.16006600 & 3.00189400\end{array}$

$\begin{array}{llll}\mathrm{H} & 4.77381000 & 3.99422200 & 3.72864400\end{array}$

$\begin{array}{llll}\mathrm{N} & 2.65825600 & 0.72542900 & 2.49539800\end{array}$

$\begin{array}{lllll}\mathrm{C} & 2.35639400 & -1.75448900 & 2.34209200\end{array}$

$\begin{array}{llll}\mathrm{C} & 3.23571800 & -2.81072900 & 2.61899500\end{array}$

$\begin{array}{llll}\mathrm{C} & 1.47220100 & -1.89442600 & 1.25482900\end{array}$

$\begin{array}{llll}\mathrm{C} & 3.24324400 & -3.97714400 & 1.85499900\end{array}$

$\begin{array}{llll}\mathrm{H} & 3.92700600 & -2.71348400 & 3.45292800\end{array}$

$\begin{array}{llll}\mathrm{C} & 1.49385600 & -3.05781400 & 0.47733300\end{array}$

$\begin{array}{llll}\text { C } & 2.36766600 & -4.10206900 & 0.77786300\end{array}$

$\begin{array}{llll}\mathrm{H} & 3.93724600 & -4.77734100 & 2.09602700\end{array}$

$\mathrm{H} \quad \begin{array}{llll}0.82710600 & -3.13179500 & -0.37632400\end{array}$

$\mathrm{H} \quad 2.37116300 \quad-4.99881400 \quad 0.16488500$

$\begin{array}{llll}\text { S } & 0.30705600 & -0.61214800 & 0.74648900\end{array}$

$\begin{array}{llll}\mathrm{C} & -1.14178900 & -1.09294600 & 1.77180800\end{array}$

$\mathrm{H} \quad-1.94391400 \quad-0.39501200 \quad 1.51916700$

$\mathrm{H} \quad-1.44643600 \quad-2.11028000 \quad 1.51526500$

$\mathrm{H} \quad-0.92749700 \quad-1.01920000 \quad 2.83991500$

$\begin{array}{llll}\mathrm{H} & 3.35056200 & 0.55560500 & 1.76106100\end{array}$

$\begin{array}{llll}\text { B } & 2.73561000 & 1.03968200 & -0.97950500\end{array}$

$\begin{array}{llll}\mathrm{H} & 2.99523300 & 1.53277600 & 0.13816600\end{array}$

$\mathrm{H} \quad 2.56678900 \quad-0.16716300 \quad-0.85597100$

$\mathrm{H} \quad 3.70604100 \quad 1.25882400 \quad-1.69477700$

$\mathrm{N} \quad \begin{array}{llll}1.39921900 & 1.71130700 & -1.45669500\end{array}$

$\mathrm{H} \quad \begin{array}{llll}\mathrm{H} & 1.55495900 & 2.62011700 & -1.89112100\end{array}$

$\mathrm{H} \quad \begin{array}{llll}0.89883900 & 1.15054600 & -2.14470900\end{array}$

TS-III

01

$\begin{array}{llll}\text { Mn } & 0.25211600 & 1.72257800 & 2.71051200\end{array}$

$\begin{array}{llll}\mathrm{O} & 0.26754700 & 3.74288900 & 4.89981700\end{array}$

$\begin{array}{llll}\mathrm{O} & -2.02015900 & 3.05757000 & 1.39733100\end{array}$

$\begin{array}{llll}\mathrm{N} & 1.66332500 & 2.59952900 & 1.51323100\end{array}$

$\begin{array}{llll}\mathrm{C} & 2.95693000 & 2.38111100 & 1.84527300\end{array}$

$\begin{array}{llll}\text { C } & 1.41447500 & 3.29017900 & 0.38174600\end{array}$

$\begin{array}{llll}\mathrm{H} & 0.36969900 & 3.44854200 & 0.14403500\end{array}$

$\begin{array}{llll}\mathrm{C} & 3.74979400 & 3.53413900 & -0.11445200\end{array}$

$\begin{array}{llll}\mathrm{H} & 4.55951300 & 3.88892400 & -0.74514100\end{array}$

$\begin{array}{lllll}\mathrm{C} & 2.41827700 & 3.77473200 & -0.44998800\end{array}$

$\mathrm{H} \quad 2.14958200 \quad 4.32462000 \quad-1.34611500$

C $\quad 3.17848700 \quad 1.66750800 \quad 3.15220600$

$\begin{array}{llll}\mathrm{H} & 4.16575200 & 1.18644200 & 3.17019100\end{array}$
$\mathrm{H}$

C

$\mathrm{H}$

$\mathrm{H}$

TS-IV

01

$\mathrm{Mn}$

$\mathrm{O}$

$\mathrm{O}$

$\mathrm{N}$

C

C

$\mathrm{H}$

C

$\mathrm{H}$

$\mathrm{C}$

C

$\mathrm{H}$

$\mathrm{H}$

$\mathrm{C}$

$\mathrm{H}$

C

$\mathrm{H}$

$\mathrm{N}$

C

n

$\begin{array}{lll}3.16399700 & 2.40802900 & 3.96422100\end{array}$

$\begin{array}{llll}2.08677300 & 0.19418600 & 4.79370500\end{array}$

$\begin{array}{lll}1.94538600 & 1.04751400 & 5.46387900\end{array}$

$\begin{array}{lll}1.19213000 & -0.42203900 & 4.89273500\end{array}$

$\begin{array}{lll}0.30501300 & 2.96987600 & 4.03448800\end{array}$

$\begin{array}{lll}-1.13579600 & 2.52824500 & 1.93958300\end{array}$

$\begin{array}{lll}4.01607400 & 2.82130500 & 1.05255100\end{array}$

$\begin{array}{lll}5.03585700 & 2.60501000 & 1.35674500\end{array}$

$\begin{array}{lll}2.07756500 & 0.72487100 & 3.40870300\end{array}$

$\begin{array}{lll}3.33730900 & -0.57017200 & 5.19982300\end{array}$

$\begin{array}{lll}4.16689500 & -0.03482900 & 6.19475700\end{array}$

$\begin{array}{lll}3.69768900 & -1.81389100 & 4.63320800\end{array}$

$\begin{array}{lll}5.32392900 & -0.69106400 & 6.61803000\end{array}$

$\begin{array}{llll}3.89483400 & 0.91598500 & 6.64752700\end{array}$

$\begin{array}{lll}4.86342500 & -2.46619300 & 5.05434300\end{array}$

$\begin{array}{lll}5.67709200 & -1.91055300 & 6.04312300\end{array}$

$\begin{array}{llll}5.94513100 & -0.24774100 & 7.39149300\end{array}$

$\begin{array}{lll}5.12575600 & -3.41663400 & 4.59943500\end{array}$

$\begin{array}{lll}6.57670800 & -2.43039700 & 6.36079500\end{array}$

$\begin{array}{lll}2.73582200 & -2.58021300 & 3.31345900\end{array}$

$\begin{array}{lll}1.38542100 & -3.39375600 & 4.25518900\end{array}$

$\begin{array}{lll}0.75349000 & -3.89064800 & 3.51448300\end{array}$

$\begin{array}{lll}1.79428600 & -4.13613500 & 4.94478300\end{array}$

$\begin{array}{lll}0.78036300 & -2.66590400 & 4.80021900\end{array}$

$\begin{array}{lll}2.16440900 & -0.07306800 & 2.77754100\end{array}$

$\begin{array}{lll}0.19303500 & 0.58633400 & 1.57499900\end{array}$

$\begin{array}{lll}-0.85409400 & 0.41820000 & 4.00160700\end{array}$

$\begin{array}{lll}-0.83065200 & -0.09332600 & 2.90701800\end{array}$

$\begin{array}{lll}-1.37104500 & -1.25430800 & 2.19581300\end{array}$

$-2.21331100 \quad-2.12475500 \quad 2.91432300$

$\begin{array}{lll}-1.08399100 & -1.53181700 & 0.85449600\end{array}$

$\begin{array}{lll}-2.74589400 & -3.25297800 & 2.29699600\end{array}$

$\begin{array}{lll}-2.44138100 & -1.89715600 & 3.95093900\end{array}$

$\begin{array}{lll}-1.62136700 & -2.66286900 & 0.23956400\end{array}$

$\begin{array}{lll}-0.44089800 & -0.85241400 & 0.30405600\end{array}$

$\begin{array}{lll}-2.45074100 & -3.52651600 & 0.95725900\end{array}$

$\begin{array}{lll}-3.39653300 & -3.91760600 & 2.85910200\end{array}$

$\begin{array}{lll}-1.39280000 & -2.86759200 & -0.80281800\end{array}$

$\begin{array}{llll}-2.86985500 & -4.40604900 & 0.47579200\end{array}$

$\begin{array}{lll}0.62013100 & 1.62743300 & 1.59141900\end{array}$

$\begin{array}{lll}-0.46340800 & 1.99334300 & 4.32508700\end{array}$

$\begin{array}{lll}-1.65699000 & 2.89219900 & 0.19449900\end{array}$

$\begin{array}{lll}1.73996900 & 3.34117100 & 1.73739400\end{array}$

$\begin{array}{lll}2.95130200 & 3.18739400 & 2.31854100\end{array}$

$\begin{array}{llll}1.40555700 & 4.55680200 & 1.26286200\end{array}$

$\begin{array}{llll}0.42828100 & 4.63344300 & 0.80102400\end{array}$

$\begin{array}{lll}3.49927500 & 5.50382100 & 1.94353600\end{array}$

$\begin{array}{lll}4.18695800 & 6.34094500 & 2.02247400\end{array}$

$\begin{array}{lll}2.24712000 & 5.65982700 & 1.34870100\end{array}$

$\begin{array}{llll}1.92255200 & 6.61549900 & 0.95013600\end{array}$

$\begin{array}{llll}3.24135800 & 1.81273700 & 2.86471700\end{array}$

$\begin{array}{lll}4.32894400 & 1.62027200 & 2.82937700\end{array}$

$\begin{array}{llll}2.96882300 & 1.80988800 & 3.93795600\end{array}$

$\begin{array}{lll}2.59557900 & -0.50412100 & 2.74987900\end{array}$

$\begin{array}{lll}3.62947300 & -0.66695900 & 3.09661800\end{array}$

$\begin{array}{lll}1.96468200 & -0.56946400 & 3.65470400\end{array}$

$\begin{array}{lll}-0.04248800 & 1.84761000 & 3.24894200\end{array}$

$\begin{array}{lll}-0.78012900 & 2.38360500 & 0.76855900\end{array}$

$\begin{array}{lll}3.85261600 & 4.24803800 & 2.42877000\end{array}$

$\begin{array}{llll}4.81996300 & 4.07896200 & 2.89225600\end{array}$

$\begin{array}{lll}2.47274400 & 0.81663600 & 2.13693400\end{array}$

$\begin{array}{llll}2.26247200 & -1.64172200 & 1.80028400\end{array}$

$\begin{array}{lll}3.22642400 & -2.63279100 & 1.56794300\end{array}$ 


$\begin{array}{lrrr}\mathrm{C} & 1.02530800 & -1.76792700 & 1.13829700 \\ \mathrm{C} & 2.98579400 & -3.71113000 & 0.71592300 \\ \mathrm{H} & 4.18842500 & -2.55183300 & 2.06870300 \\ \mathrm{C} & 0.78675800 & -2.84021700 & 0.27032100 \\ \mathrm{C} & 1.76016200 & -3.81697600 & 0.06090000 \\ \mathrm{H} & 3.75800100 & -4.45961200 & 0.56002900 \\ \mathrm{H} & -0.16838500 & -2.90053200 & -0.24320700 \\ \mathrm{H} & 1.56229100 & -4.64579300 & -0.61296800 \\ \mathrm{~S} & -0.30533500 & -0.56132300 & 1.30867800 \\ \mathrm{C} & -1.09707100 & -1.17143800 & 2.84921700 \\ \mathrm{H} & -1.95714800 & -0.52384100 & 3.03606400 \\ \mathrm{H} & -1.43518300 & -2.19981700 & 2.70206700 \\ \mathrm{H} & -0.41316200 & -1.11345500 & 3.69832300 \\ \mathrm{H} & 2.50366300 & 0.88776600 & 0.82250500 \\ \mathrm{~N} & 1.68723400 & 1.24897600 & -0.09564600 \\ \mathrm{C} & 1.66160400 & 1.34946900 & -1.36058000 \\ \mathrm{H} & 0.76343700 & 1.74666000 & -1.86343200 \\ \mathrm{C} & 2.75481100 & 0.97929500 & -2.29428500 \\ \mathrm{C} & 2.59310600 & 1.23131400 & -3.66501400 \\ \mathrm{C} & 3.94793400 & 0.38265200 & -1.85391500 \\ \mathrm{C} & 3.59783100 & 0.90493600 & -4.57616500 \\ \mathrm{H} & 1.66976800 & 1.68943300 & -4.01380200 \\ \mathrm{C} & 4.95063000 & 0.05448200 & -2.76189500 \\ \mathrm{H} & 4.07217900 & 0.17346000 & -0.79545500 \\ \mathrm{C} & 4.78068100 & 0.31577100 & -4.12629500 \\ \mathrm{H} & 3.45755900 & 1.10889200 & -5.63454000 \\ \mathrm{H} & 5.86852900 & -0.40925400 & -2.40920800 \\ \mathrm{H} & 5.56553800 & 0.05837900 & -4.83278200\end{array}$

TS-V

$\begin{array}{lrrr}0 \mathrm{1} & & & \\ \mathrm{Mn} & 1.11278100 & 1.13074200 & 1.01765000 \\ \mathrm{O} & -0.15685200 & 0.55560400 & 3.62795000 \\ \mathrm{O} & -1.37970200 & 2.35822300 & 0.02747100 \\ \mathrm{~N} & 1.97111300 & 2.82552700 & 1.76550100 \\ \mathrm{C} & 3.10129400 & 2.62735800 & 2.48695700 \\ \mathrm{C} & 1.52304400 & 4.08694600 & 1.61285000 \\ \mathrm{H} & 0.62443000 & 4.20091700 & 1.01822000 \\ \mathrm{C} & 3.31886900 & 4.98332600 & 2.92621500 \\ \mathrm{H} & 3.84528000 & 5.81920500 & 3.37709600 \\ \mathrm{C} & 2.16009000 & 5.18682600 & 2.17843300 \\ \mathrm{H} & 1.75180000 & 6.18012200 & 2.02433100 \\ \mathrm{C} & 3.54811300 & 1.18935700 & 2.60219500 \\ \mathrm{H} & 4.63821600 & 1.13696700 & 2.74307500 \\ \mathrm{H} & 3.07609900 & 0.71408200 & 3.47148600 \\ \mathrm{C} & 3.47180100 & -0.96768300 & 1.35443100 \\ \mathrm{H} & 3.51079500 & -1.21844100 & 0.28821700 \\ \mathrm{H} & 4.48351400 & -1.10932700 & 1.75906800 \\ \mathrm{C} & 0.38468100 & 0.75262100 & 2.61708800 \\ \mathrm{C} & -0.40957100 & 1.86481600 & 0.43744900 \\ \mathrm{C} & 3.79756000 & 3.68248700 & 3.07309800 \\ \mathrm{H} & 4.70673200 & 3.48043100 & 3.63094800 \\ \mathrm{~N} & 3.10824300 & 0.47471000 & 1.39254600 \\ \mathrm{C} & 2.51041400 & -1.88985900 & 2.06789900 \\ \mathrm{C} & 2.93675700 & -2.63010500 & 3.17359200 \\ \mathrm{C} & 1.18922500 & -2.07342500 & 1.60087600 \\ \mathrm{C} & 2.09057000 & -3.53590200 & 3.81654800 \\ \mathrm{H} & 3.95575700 & -2.49711000 & 3.53014400 \\ \mathrm{C} & 0.34236700 & -2.98272400 & 2.23896900 \\ \mathrm{C} & 0.79328900 & -3.71118900 & 3.34322000 \\ \mathrm{H} & 2.44521600 & -4.09854500 & 4.67507000 \\ \mathrm{H} & -0.67172400 & -3.13480800 & 1.88845700 \\ \mathrm{H} & 0.12137400 & -4.41372400 & 3.82831200 \\ \mathrm{~S} & 0.68422700 & -1.07778700 & 0.18630600 \\ \mathrm{C} & -1.10929300 & -1.39305300 & 0.08756100 \\ \mathrm{H} & -1.47523800 & -0.72662500 & -0.69650200\end{array}$

$\begin{array}{lll}-1.30480800 & -2.42838900 & -0.20155500\end{array}$ $\begin{array}{lll}-1.60609800 & -1.14889700 & 1.02944700\end{array}$ $\begin{array}{llll}3.57032300 & 0.89257900 & 0.57838500\end{array}$ $\begin{array}{llll}3.67128600 & 2.57742800 & -1.10606300\end{array}$ $\begin{array}{llll}3.68509100 & 3.27329800 & -1.84729400\end{array}$ $\begin{array}{llll}3.47545500 & 3.10054700 & -0.25758800\end{array}$ $\begin{array}{llll}2.65450700 & 1.48009600 & -1.42247600\end{array}$ $\begin{array}{llll}3.06631500 & 0.33550500 & -1.24993000\end{array}$ $\begin{array}{llll}1.62666500 & 1.56199200 & -0.57271400\end{array}$ $\begin{array}{lll}2.10511400 & 1.58305900 & -2.50215000\end{array}$

\section{TS-VI}

0

$\begin{array}{llll}\mathrm{Mn} & 0.82237900 & 1.49349100 & 1.69911100 \\ \mathrm{O} & -0.78992200 & 1.79402400 & 4.16365900\end{array}$

$\mathrm{O}$

$\mathrm{O}$

$\mathrm{N}$

C

$\mathrm{H}$

$\mathrm{H}$

C

$\mathrm{H}$

$\mathrm{C}$

$\mathrm{H}$

$\mathrm{H}$

$\mathrm{C}$

$\mathrm{H}$

C

C

$\mathrm{H}$

N

C

C

C

C

$\mathrm{H}$

C

C

$\mathrm{H}$

$\mathrm{H}$

$\mathrm{C}$

$\mathrm{H}$

$\mathrm{H}$

$\mathrm{H}$

$\mathrm{H}$

$\mathrm{H}$

$\mathrm{N}$

C

C

C

C

C

C

$\mathrm{H}$

$\mathrm{H}$

$\mathrm{H}$

$\mathrm{H}$

$\mathrm{H}$ $\begin{array}{lll}-1.23086900 & 2.72880100 & -0.02029300\end{array}$

$\begin{array}{lll}1.88586000 & 3.21143700 & 1.97551500\end{array}$

$\begin{array}{lll}2.93157900 & 3.10640900 & 2.82706700\end{array}$

$\begin{array}{lll}1.69108700 & 4.39111700 & 1.35353300\end{array}$

$\begin{array}{lll}0.84708300 & 4.43631000 & 0.67583400\end{array}$

$\begin{array}{lll}3.59496300 & 5.38286300 & 2.42489600\end{array}$

$\begin{array}{lll}4.26271500 & 6.22189800 & 2.59655400\end{array}$

$\begin{array}{lll}2.51298500 & 5.49462700 & 1.55194900\end{array}$

$\begin{array}{lll}2.30555700 & 6.41834500 & 1.02172400\end{array}$

$\begin{array}{lll}4.09822400 & 1.58290000 & 3.80934300\end{array}$

$\begin{array}{lll}2.44277800 & 1.76877800 & 4.42224200\end{array}$

$\begin{array}{lll}2.48223400 & -0.59319800 & 3.28094900\end{array}$

$\begin{array}{lll}3.37253400 & -0.73670000 & 3.91059900\end{array}$

$\begin{array}{lll}1.61349300 & -0.58504700 & 3.95027800\end{array}$

$\begin{array}{lll}-0.13479500 & 1.67271300 & 3.20540300\end{array}$

$\begin{array}{lll}-0.43749800 & 2.23078100 & 0.67554600\end{array}$

$\begin{array}{lll}3.80547400 & 4.16531100 & 3.06728900\end{array}$

$\begin{array}{lll}4.64037600 & 4.02689200 & 3.74738600\end{array}$

$\begin{array}{llll}2.54545800 & 0.72434700 & 2.60773600\end{array}$

$\begin{array}{lll}2.41423100 & -1.74286500 & 2.29797000\end{array}$

$\begin{array}{lll}3.41545000 & -2.72178300 & 2.33166000\end{array}$

$\begin{array}{lll}1.38789100 & -1.87815000 & 1.34369000\end{array}$

$\begin{array}{lll}3.40841300 & -3.80540100 & 1.45344800\end{array}$

$\begin{array}{lll}4.21967600 & -2.62548600 & 3.05695800\end{array}$

$\begin{array}{lll}1.38929600 & -2.95571400 & 0.45083200\end{array}$

$\begin{array}{llll}2.39233400 & -3.92352300 & 0.50669600\end{array}$

$\begin{array}{llll}4.20137200 & -4.54627700 & 1.50336000\end{array}$

$\begin{array}{llll}0.59957700 & -3.02714900 & -0.29129100\end{array}$

$\begin{array}{llll}2.38163900 & -4.75646500 & -0.19058800\end{array}$

$\begin{array}{lll}0.05131100 & -0.67884100 & 1.17223100\end{array}$

$\begin{array}{lll}-1.12084900 & -1.34164100 & 2.42564000\end{array}$

$\begin{array}{lll}-1.40040000 & -2.36420700 & 2.16095500\end{array}$

$\begin{array}{lll}-0.69223700 & -1.31264800 & 3.42982600\end{array}$

$\begin{array}{lll}3.22831800 & 0.65655900 & 1.81854100\end{array}$

$\begin{array}{lll}1.74996300 & 1.28669300 & 0.26796500\end{array}$

$\begin{array}{lll}4.09741700 & 0.84774200 & 0.25769500\end{array}$

$\begin{array}{llll}3.17917600 & 1.10916300 & -0.45817300\end{array}$

$\begin{array}{llll}2.83167700 & 1.35274800 & -1.86474800\end{array}$

$\begin{array}{llll}1.52473100 & 1.56166200 & -2.31635100\end{array}$

$\begin{array}{llll}3.89227300 & 1.35932200 & -2.79052000\end{array}$

$\begin{array}{llll}1.27825500 & 1.77679200 & -3.67315100\end{array}$

$\begin{array}{lll}0.71107000 & 1.55631700 & -1.60166400\end{array}$

$3.64057000 \quad 1.57652700 \quad-4.14205200$

$\begin{array}{llll}4.90194800 & 1.19253000 & -2.42916500\end{array}$

$\begin{array}{llll}2.33177200 & 1.78609000 & -4.58844000\end{array}$

$\begin{array}{llll}0.25865100 & 1.93809300 & -4.01273700\end{array}$

$\begin{array}{llll}4.46603300 & 1.58173800 & -4.84911900\end{array}$

$\begin{array}{llll}2.13661900 & 1.95539000 & -5.64422100\end{array}$
$3.05638400 \quad 1.77162800 \quad 3.51086400$

$\begin{array}{lll}-2.00320100 & -0.69778100 & 2.39418600\end{array}$

$\begin{array}{llll}0.59957700 & -3.02714900 & -0.29129100\end{array}$ 


$\begin{array}{ccc}2.38163900 & -4.75646500 & -0.19058800 \\ 0.05131100 & -0.67884100 & 1.17223100 \\ -1.12084900 & -1.34164100 & 2.42564000 \\ -2.00320100 & -0.69778100 & 2.39418600 \\ -1.40040000 & -2.36420700 & 2.16095500 \\ -0.69223700 & -1.31264800 & 3.42982600 \\ 3.22831800 & 0.65655900 & 1.81854100 \\ 1.74996300 & 1.28669300 & 0.26796500 \\ 4.09741700 & 0.84774200 & 0.25769500 \\ 3.17917600 & 1.10916300 & -0.45817300 \\ 2.83167700 & 1.35274800 & -1.86474800 \\ 1.52473100 & 1.56166200 & -2.31635100 \\ 3.89227300 & 1.35932200 & -2.79052000 \\ 1.27825500 & 1.77679200 & -3.67315100 \\ 0.71107000 & 1.55631700 & -1.60166400 \\ 3.64057000 & 1.57652700 & -4.14205200 \\ 4.90194800 & 1.19253000 & -2.42916500 \\ 2.33177200 & 1.78609000 & -4.58844000 \\ 0.25865100 & 1.93809300 & -4.01273700 \\ 4.46603300 & 1.58173800 & -4.84911900 \\ 2.13661900 & 1.95539000 & -5.64422100\end{array}$




\section{Copies of IR data of metal complexes.}

\section{IR spectrum of Mn1.}

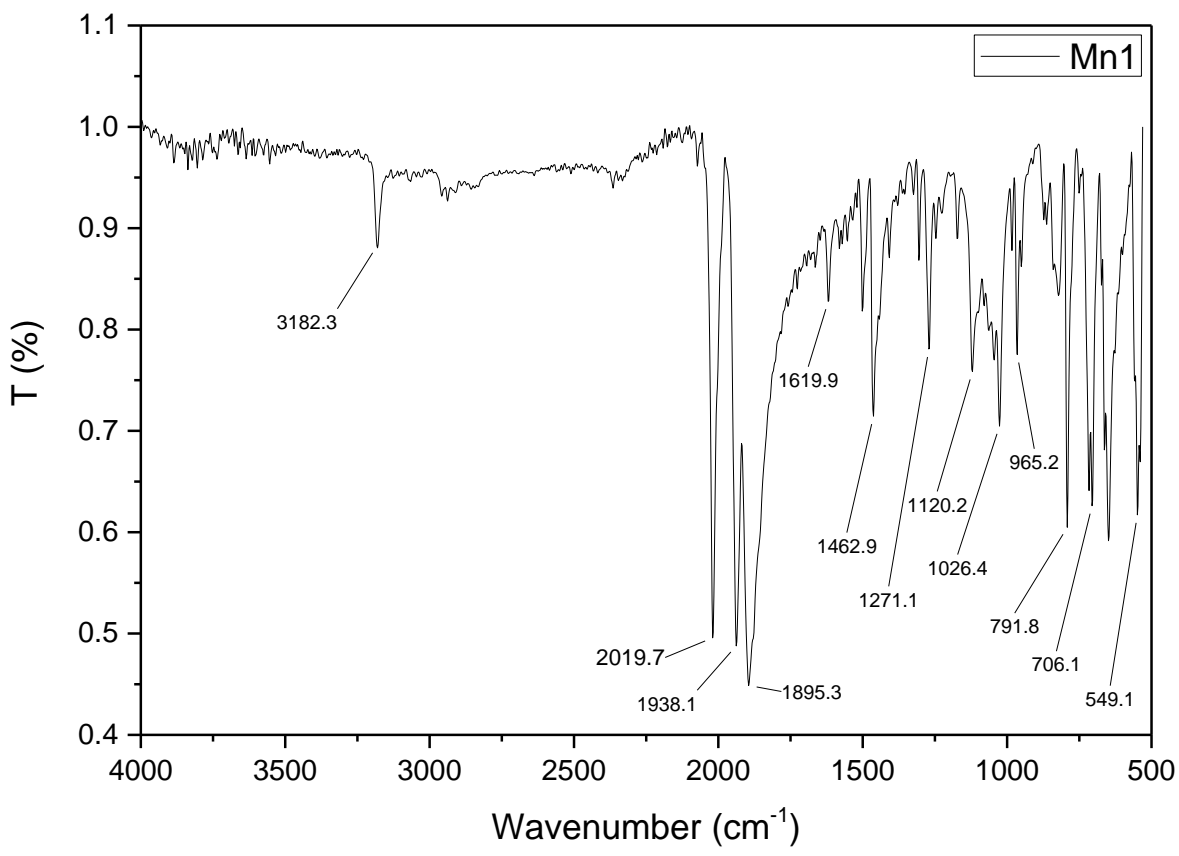

\section{IR spectrum of Mn2.}

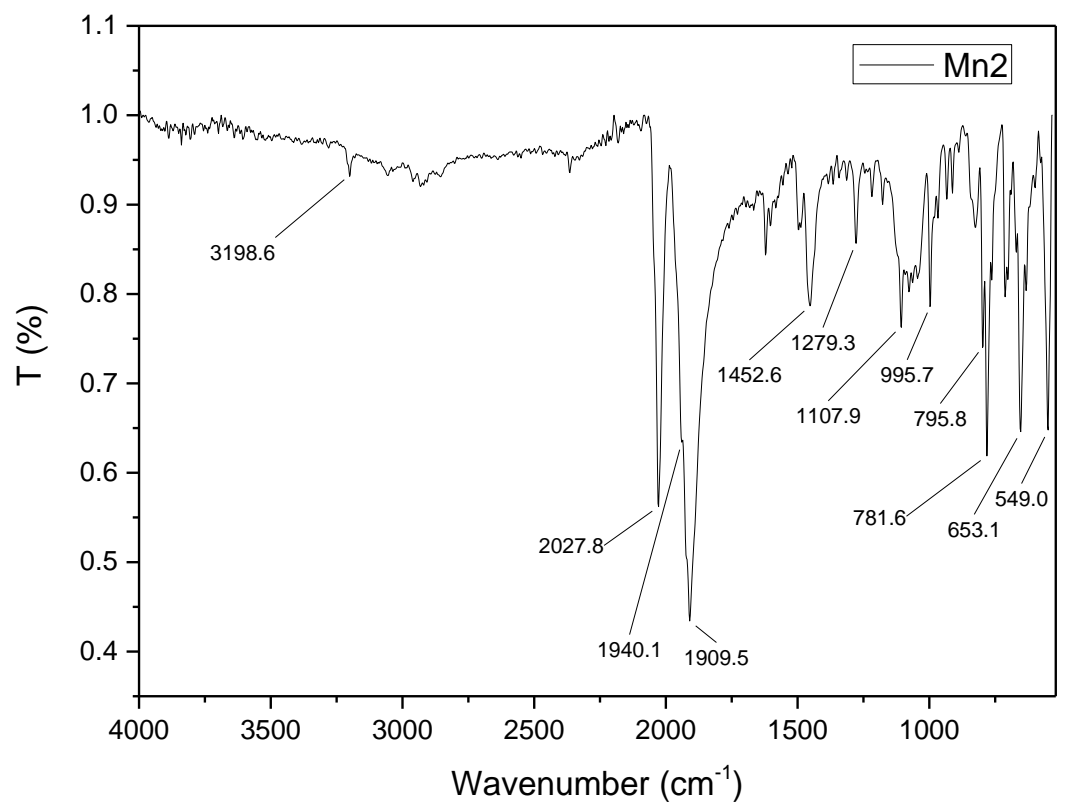




\section{IR spectrum of Mn3.}

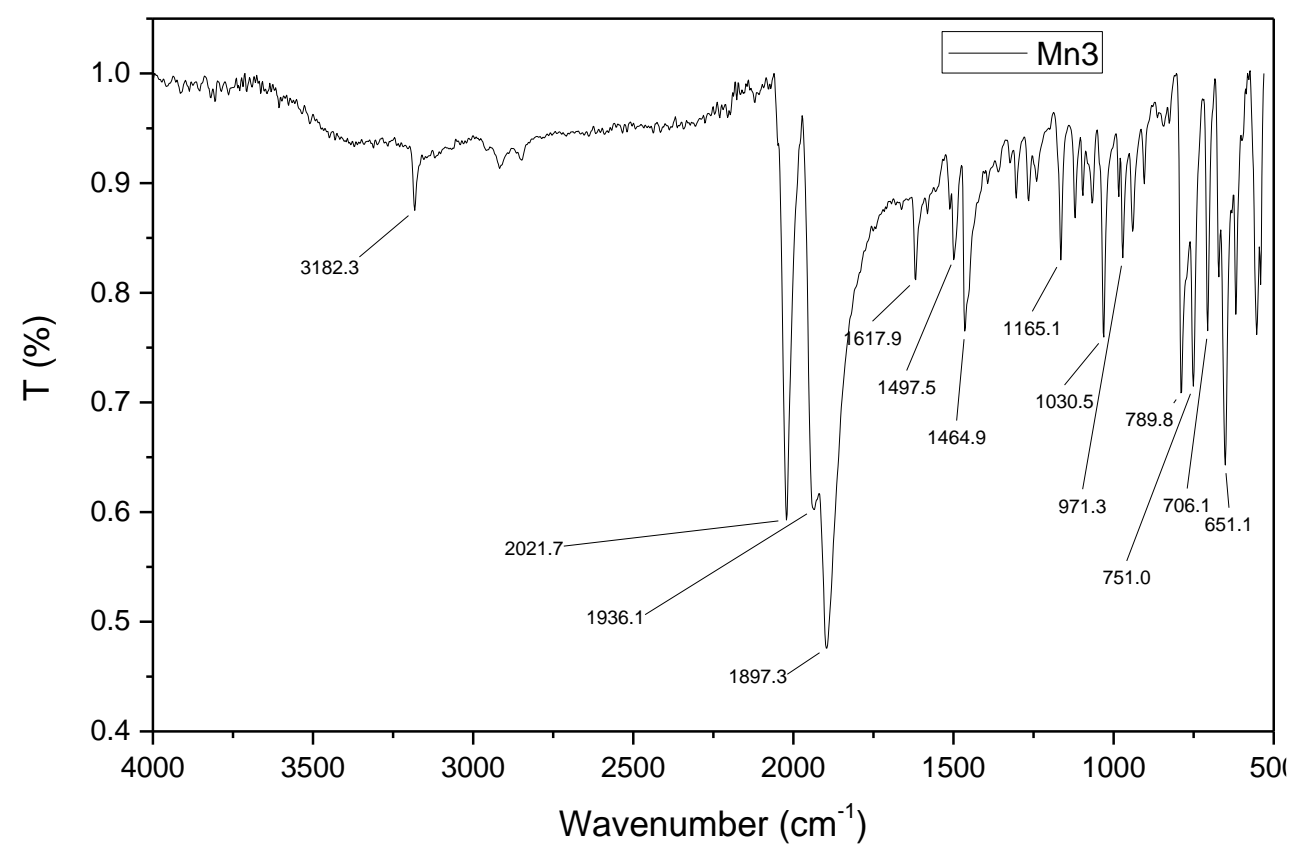

IR spectrum of Mn4.

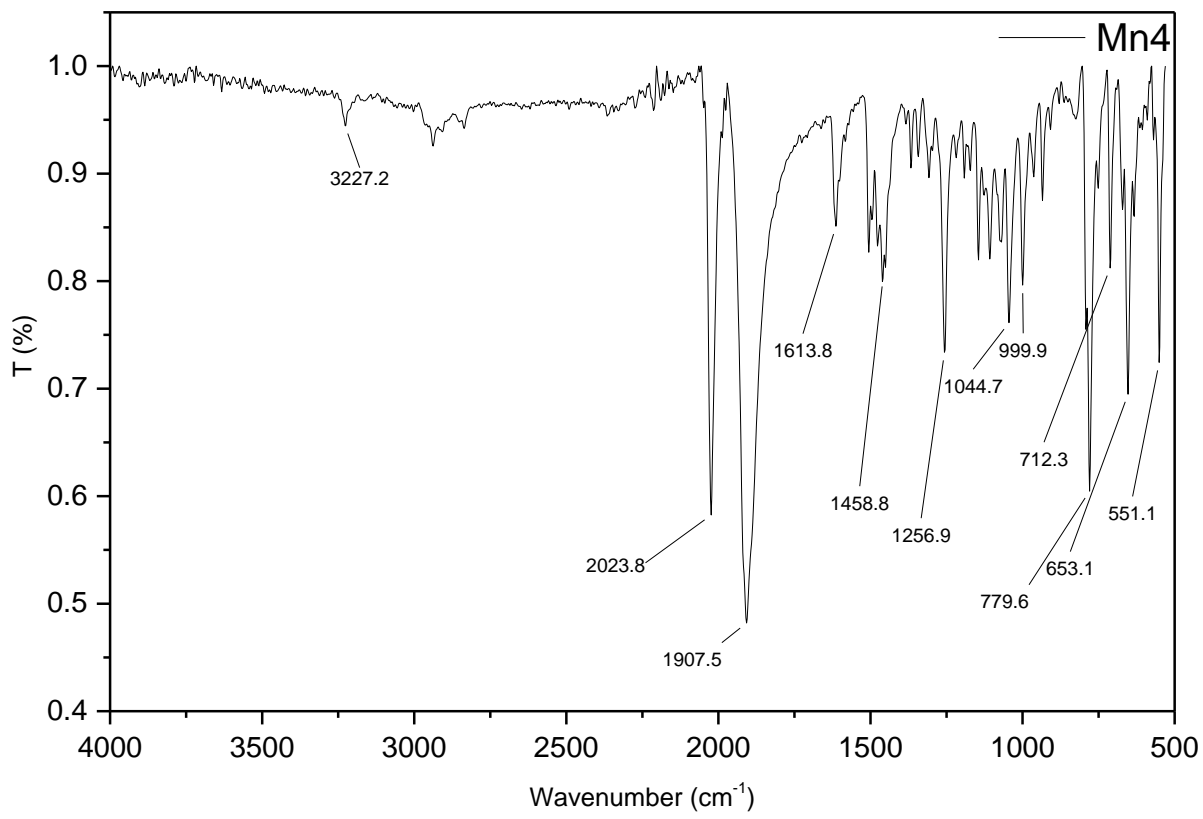




\section{Copies of GCMS traces}

11a. GCMS traces for an authentic samples of benzyl amines, dibenzyl amine, and tribenzylamine
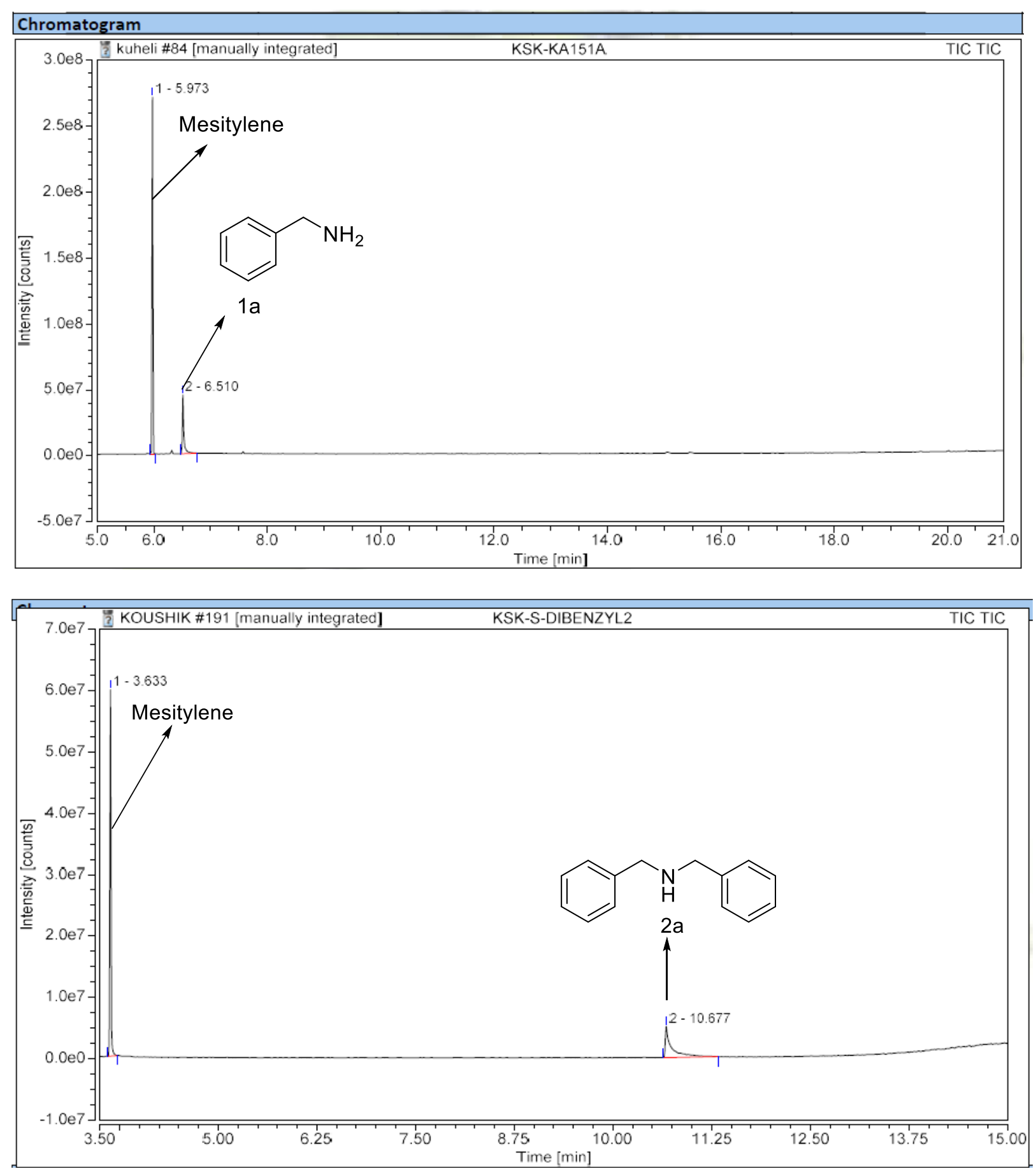


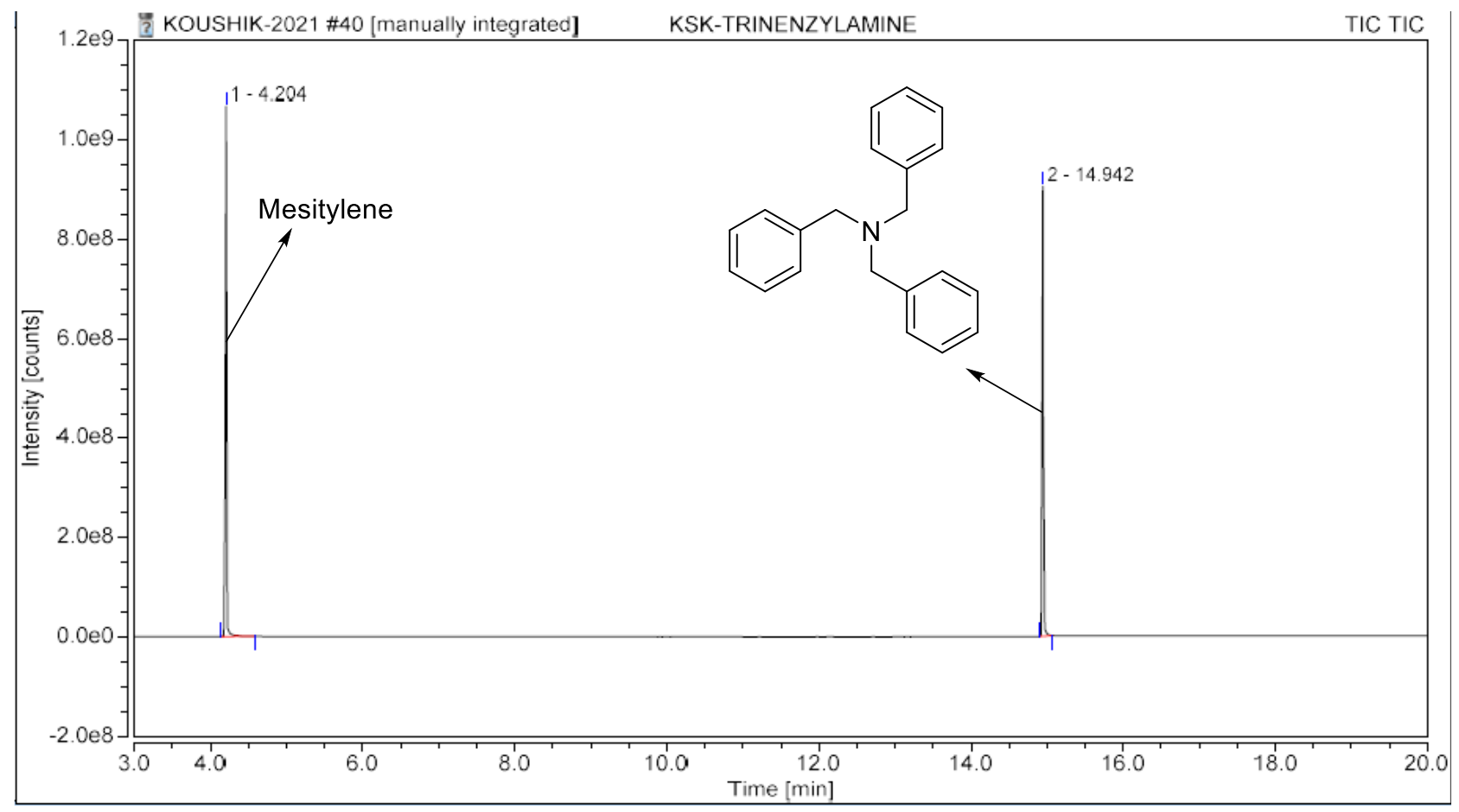

11b. Representative GCMS traces for the reaction optimization

Table 1, Entry 1

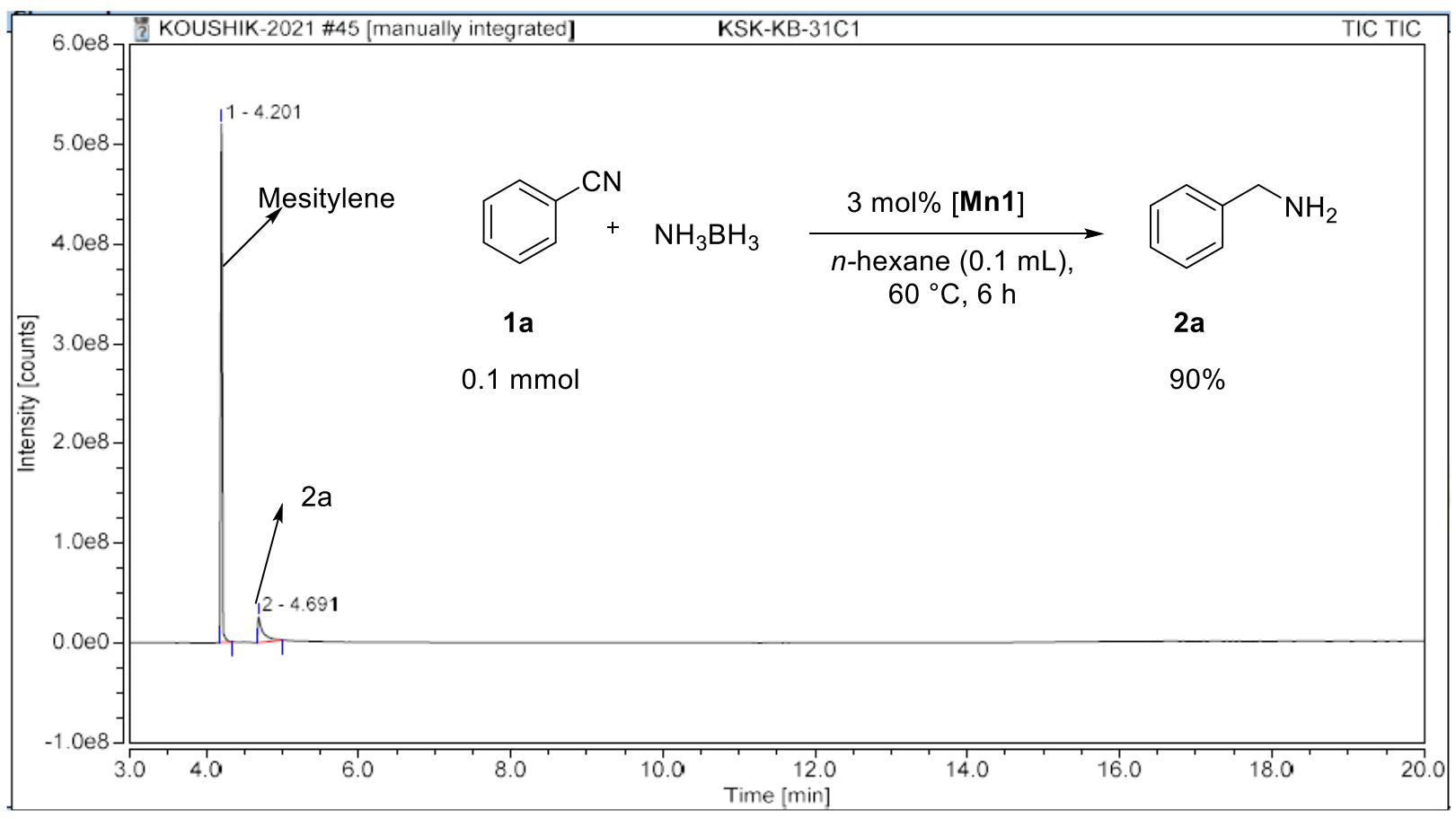


Table 1, Entry 2

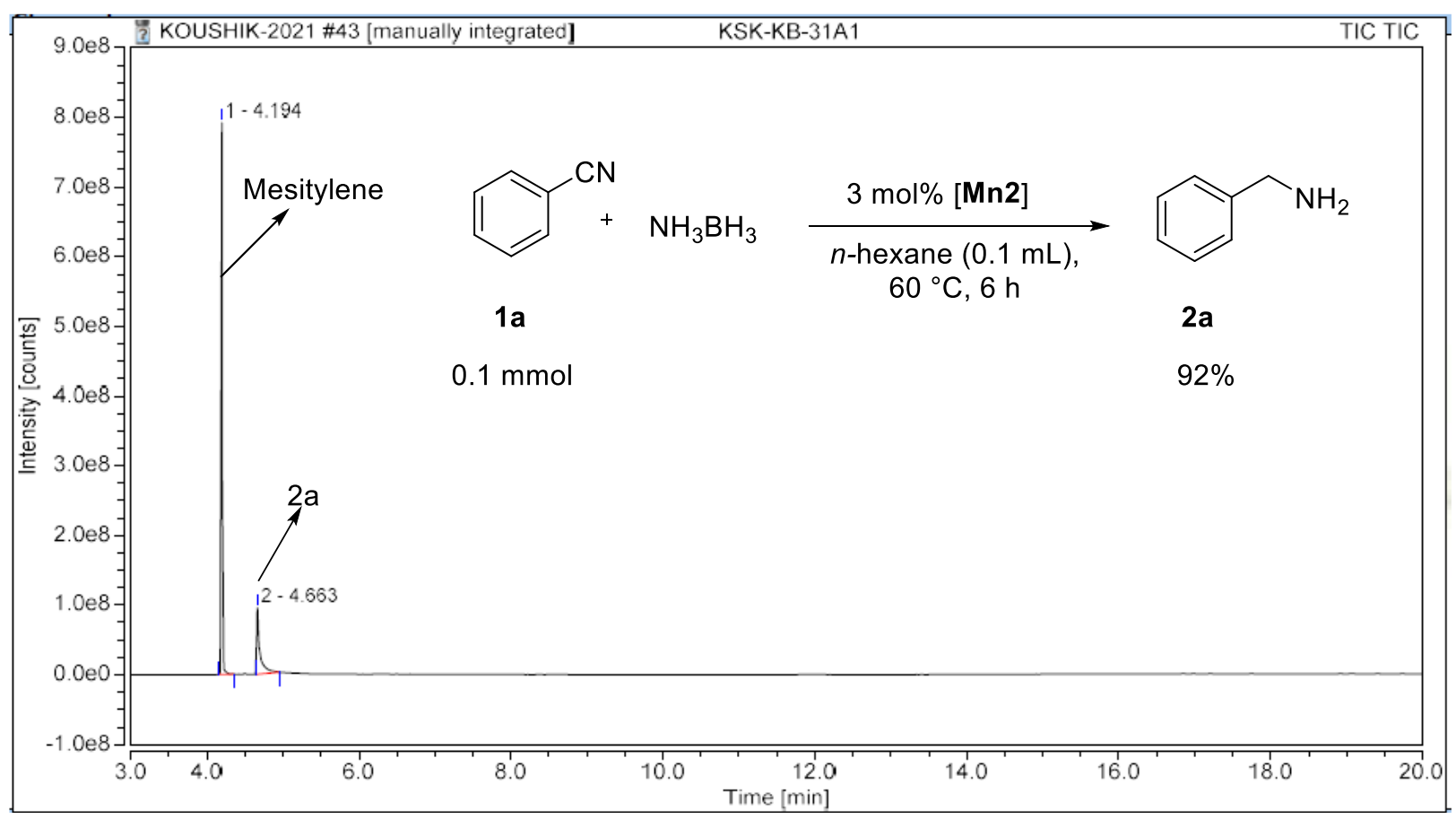

Table 1, Entry 3

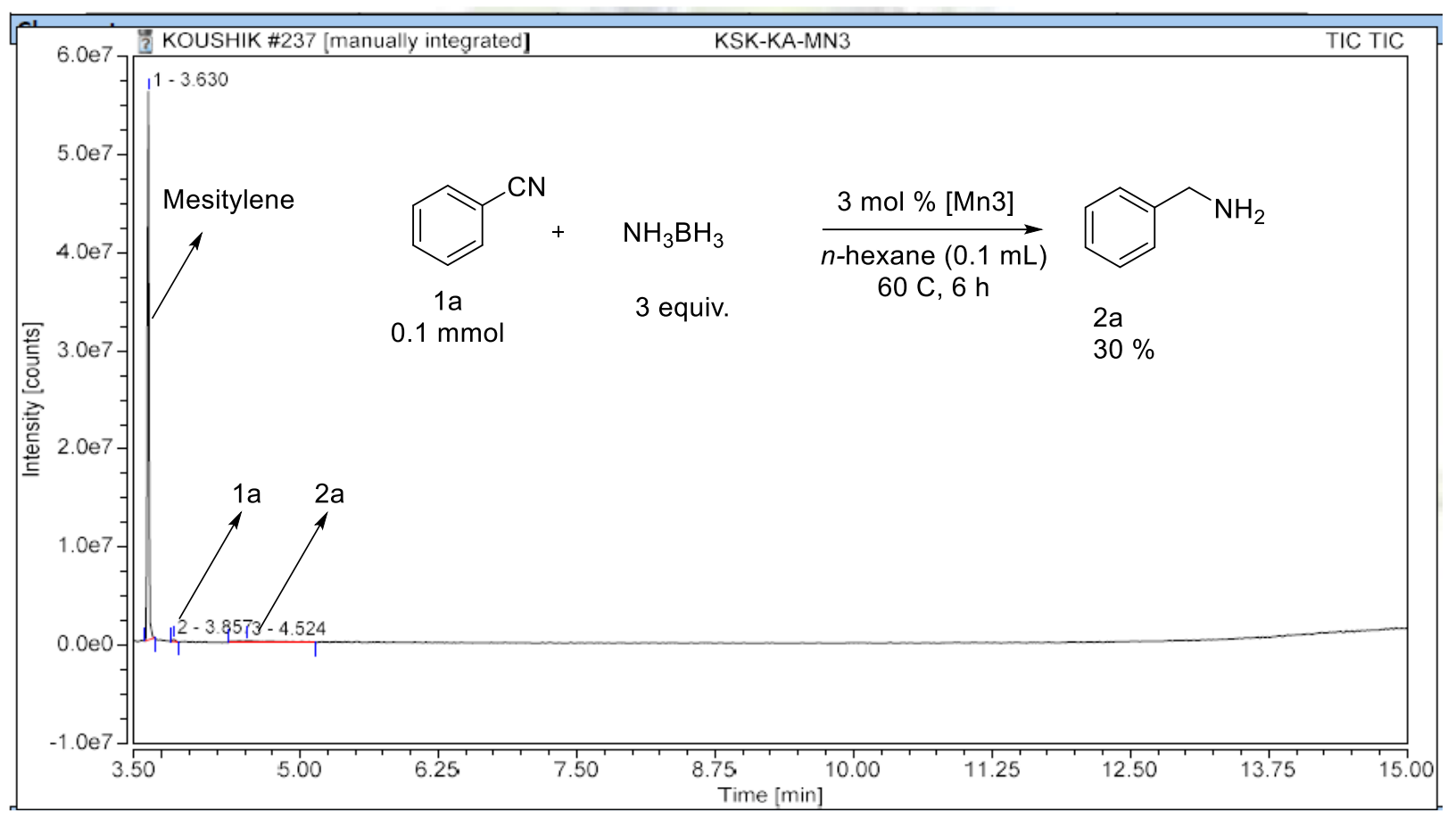


Table 1, Entry 4

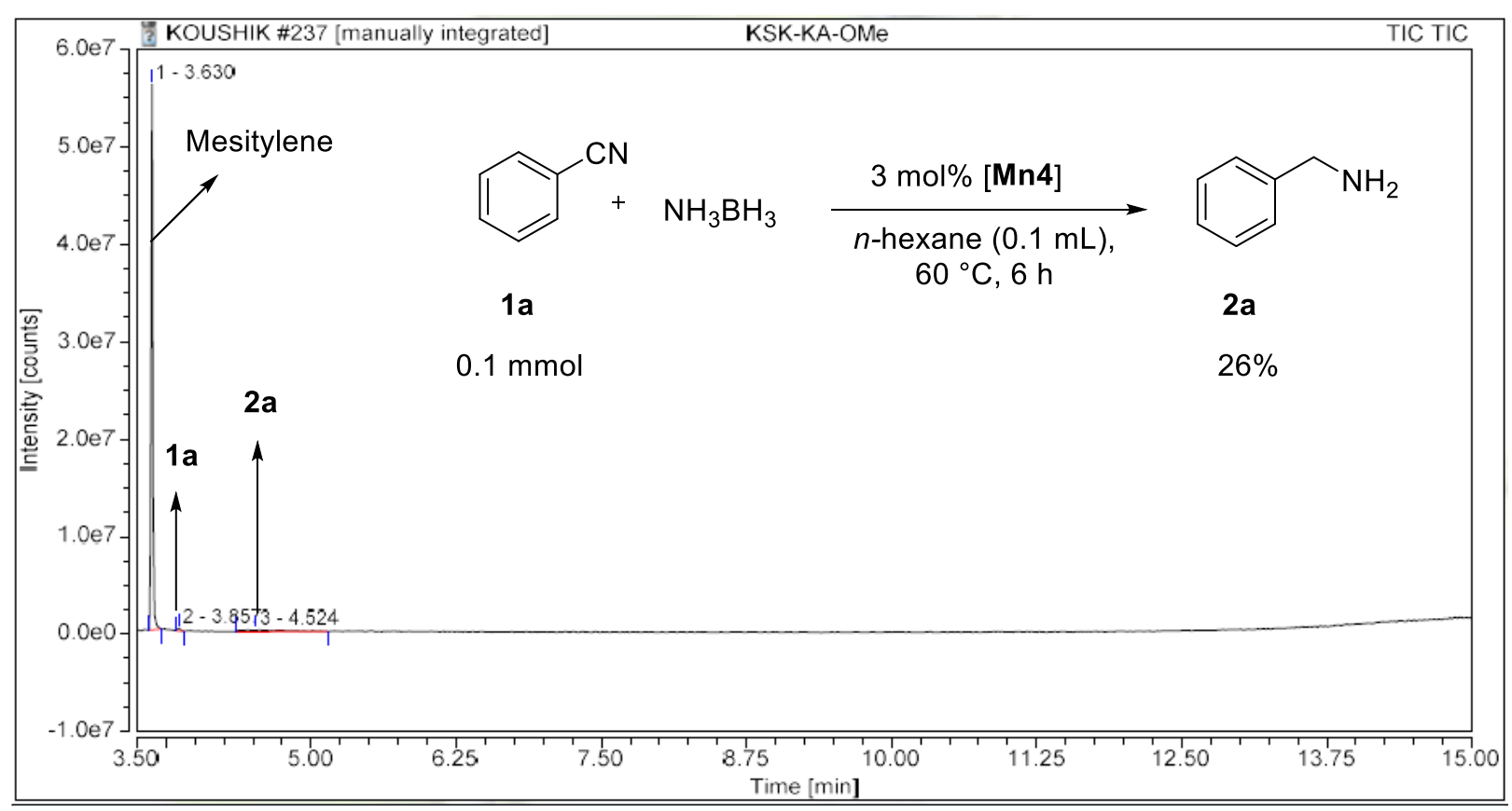

Table 1, Entry 5

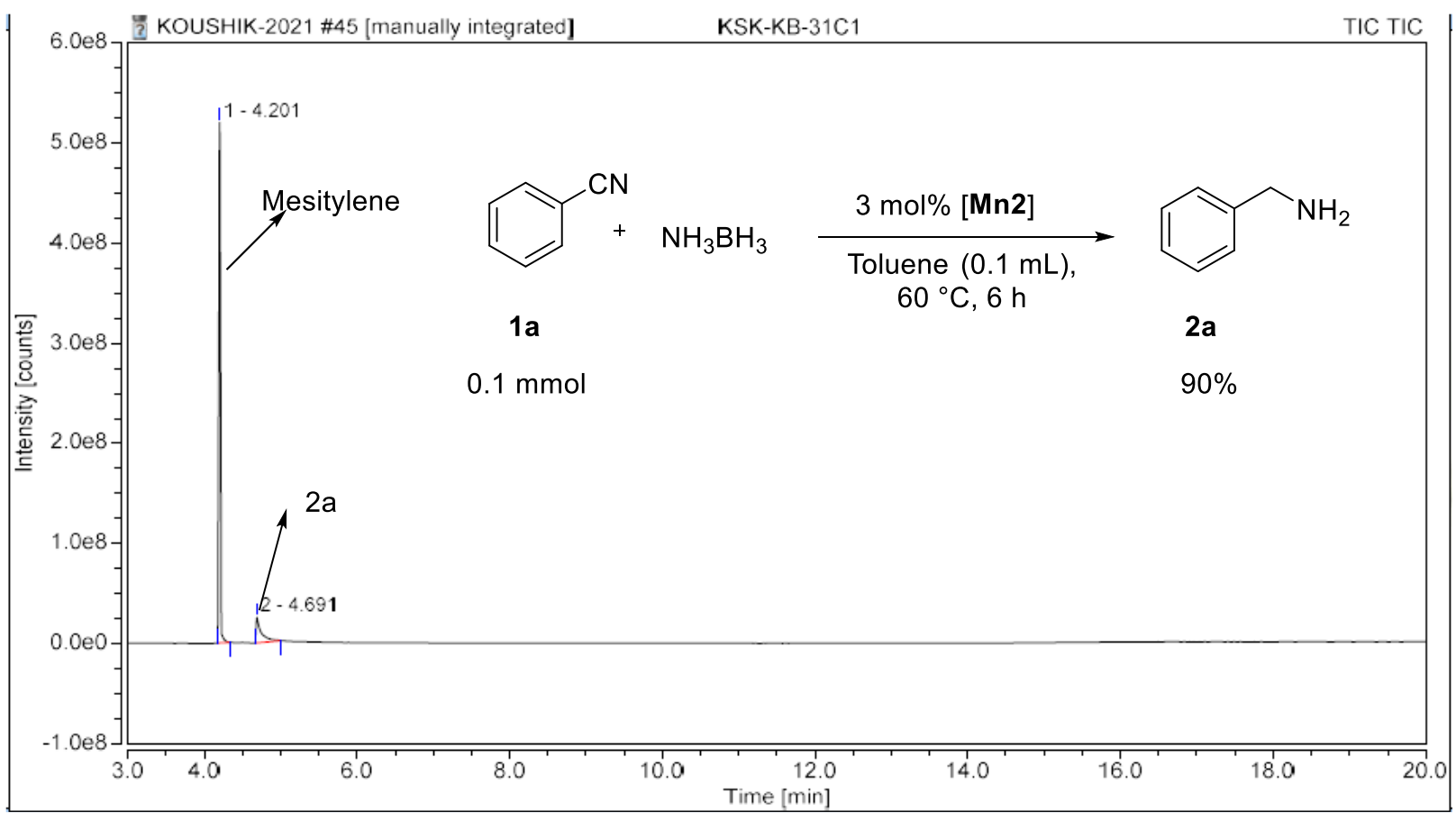


Table 1, Entry 6

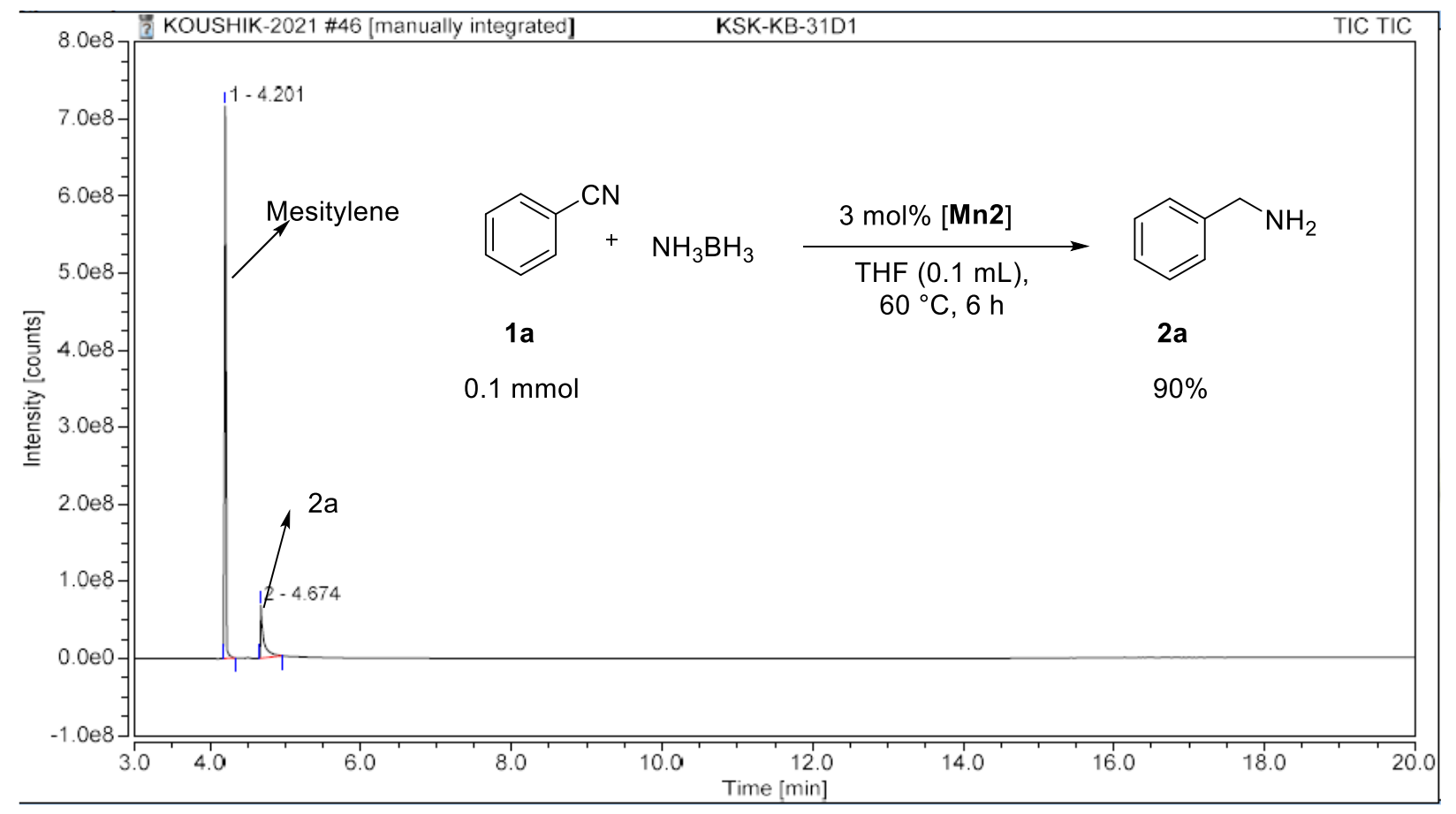

Table 1, Entry 7

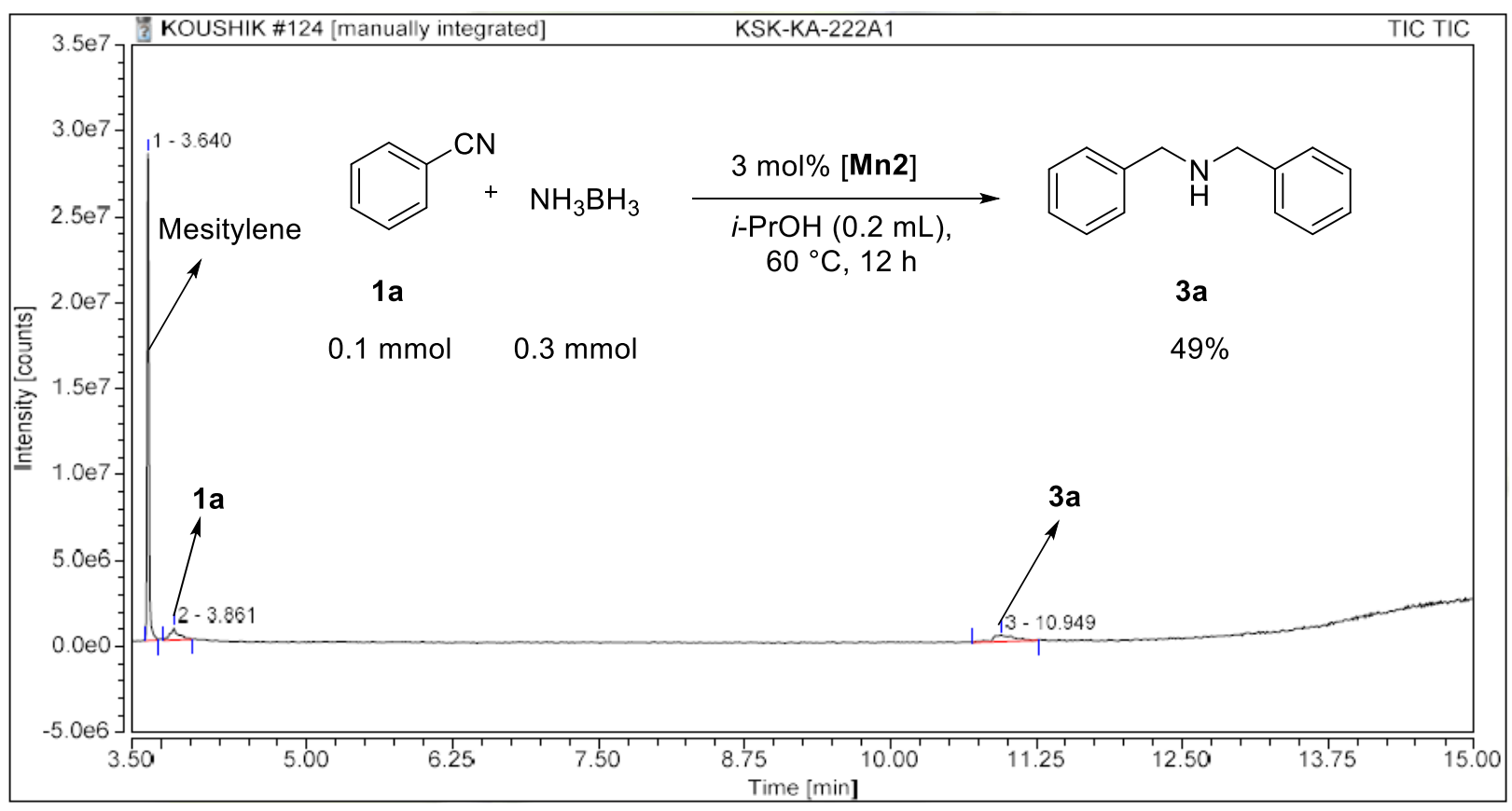


Table 1, Entry 8

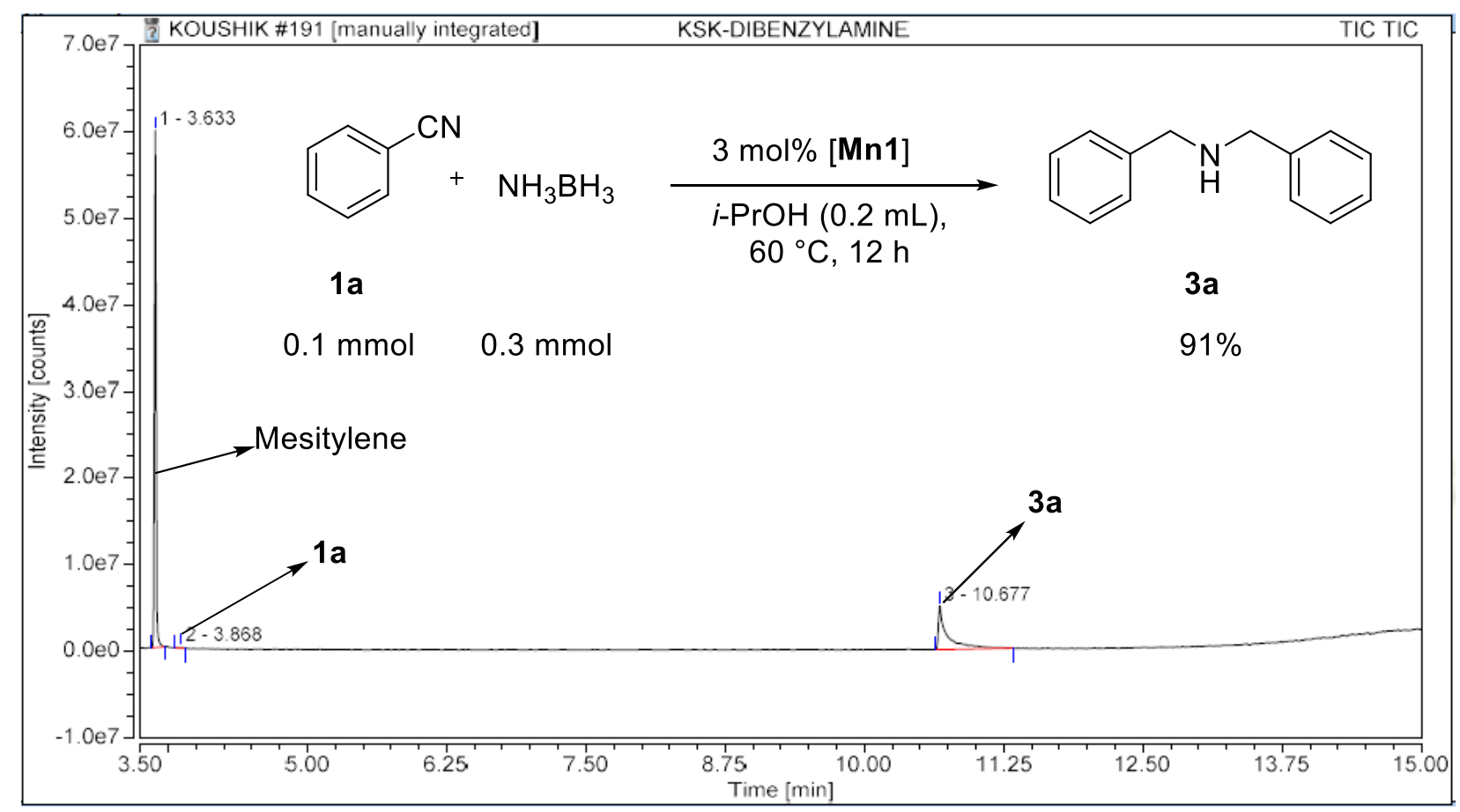

Table 1, Entry 9

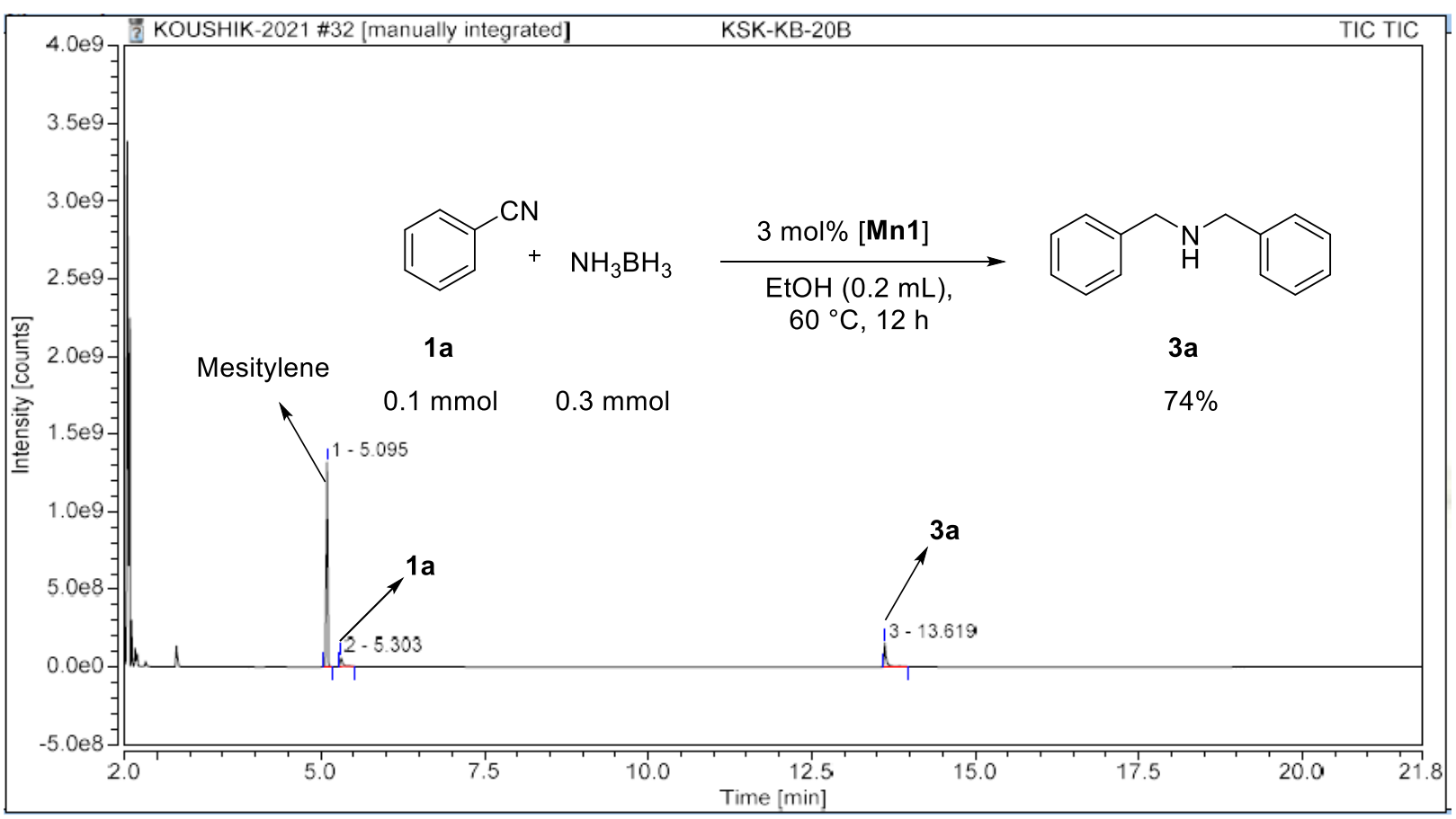


Table 1, Entry 10

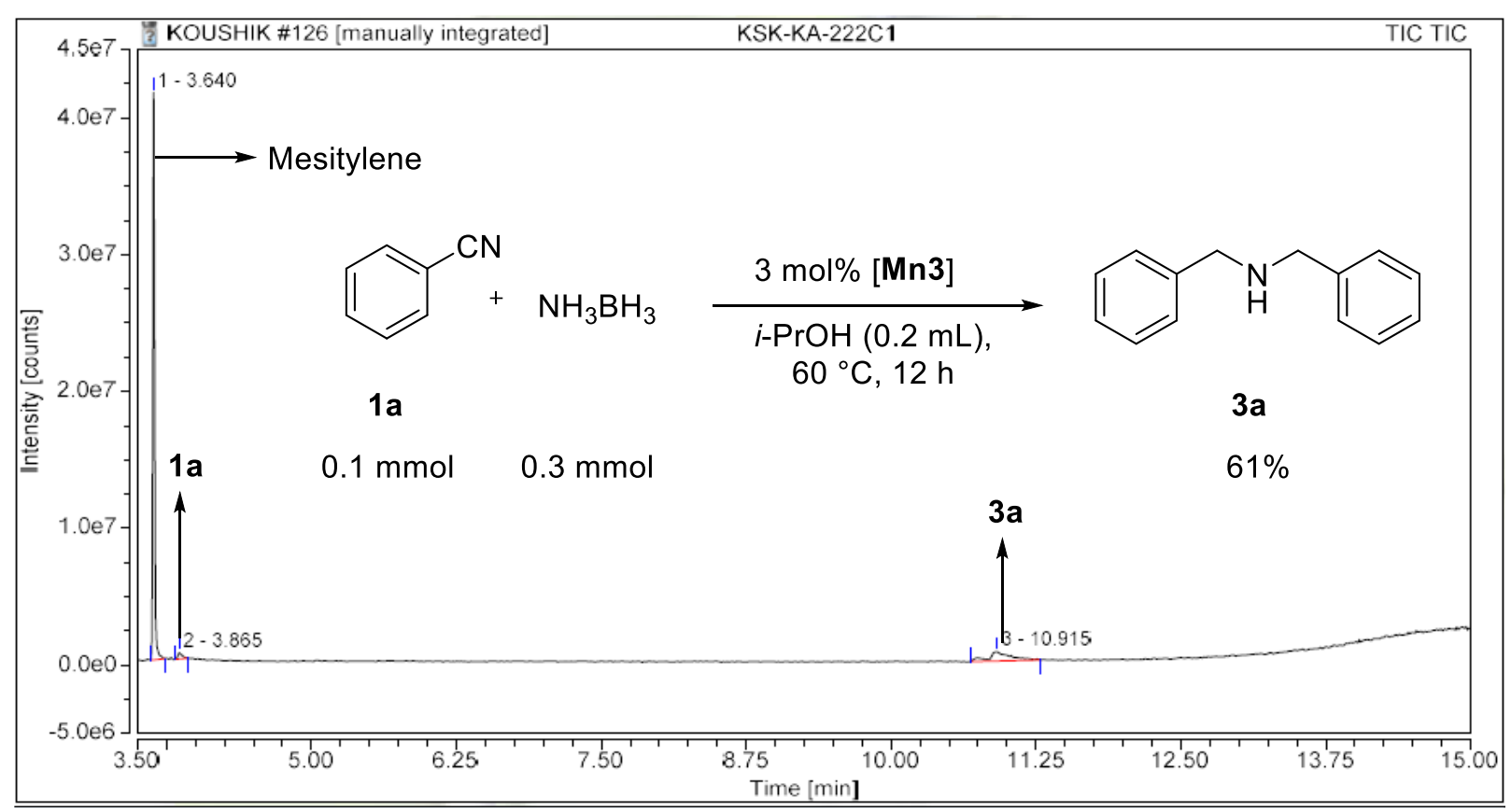

Table 1, Entry 11

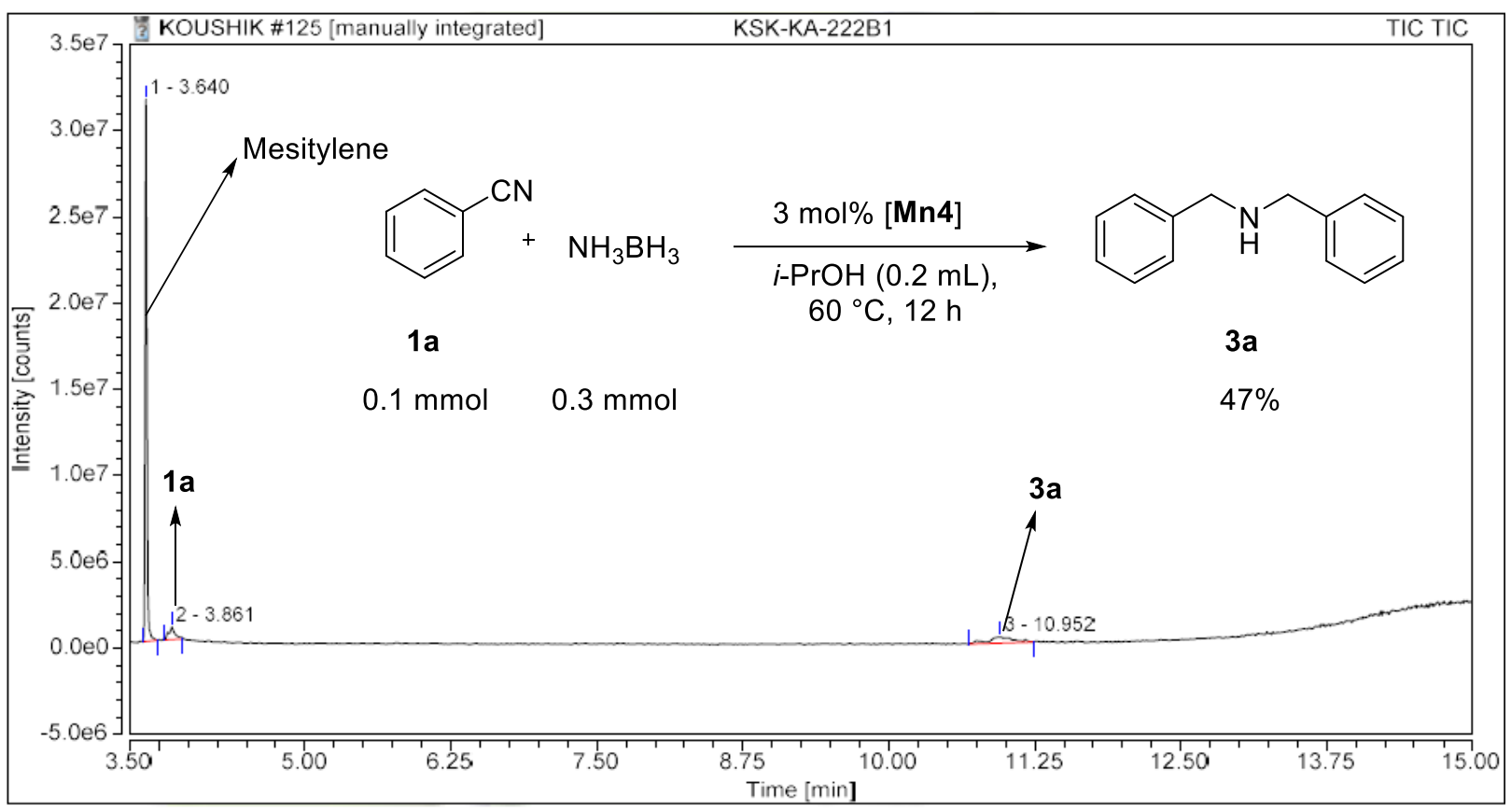




\section{Table S6, Entry 1}

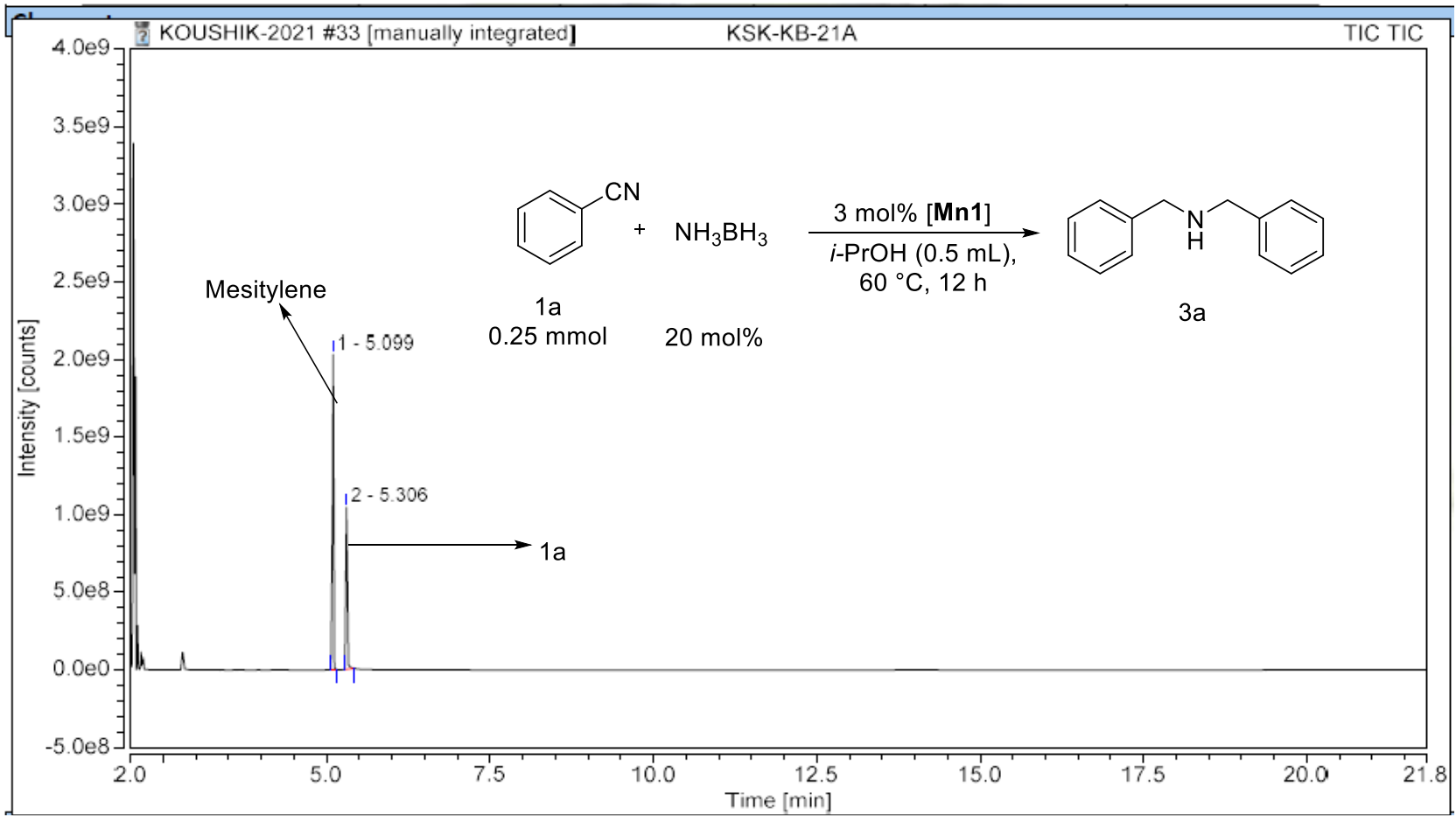

Table S7, Entry 1

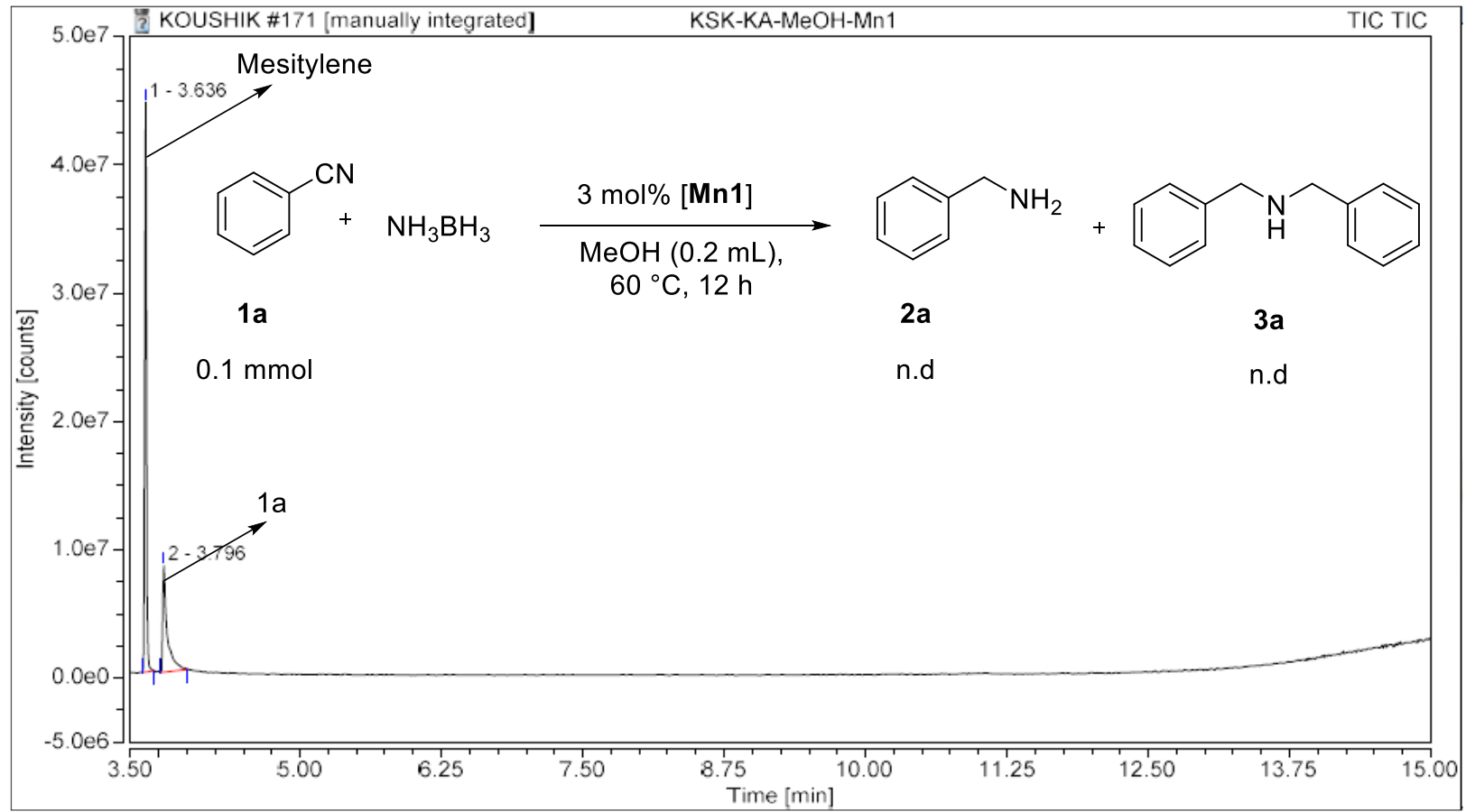


12. Copies of ${ }^{1} \mathrm{H}$ and ${ }^{13} \mathrm{C}$ NMR spectra
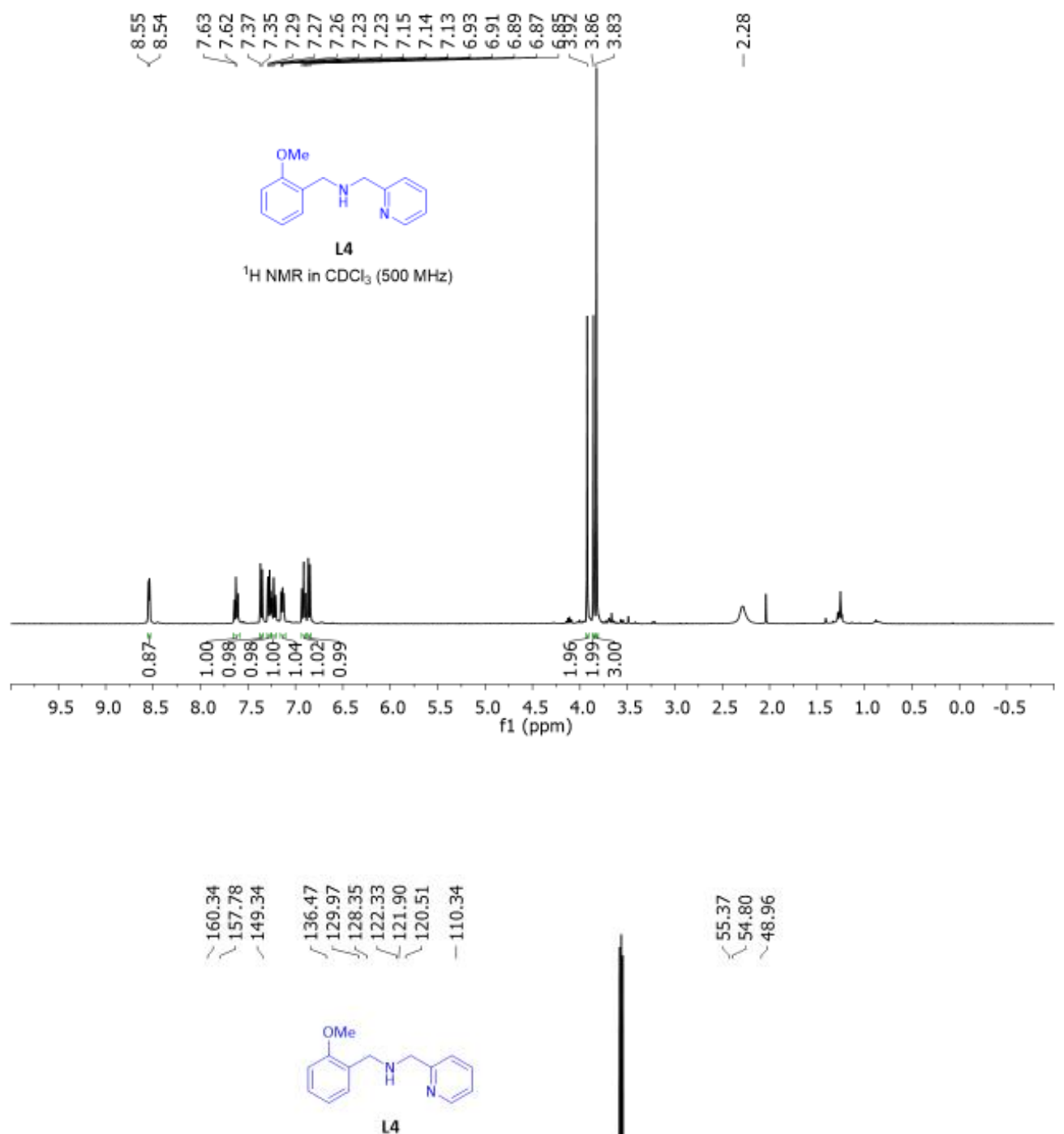

${ }^{13} \mathrm{C} \mathrm{NMR} \mathrm{in} \mathrm{CDCl}_{3}(126 \mathrm{MHz})$

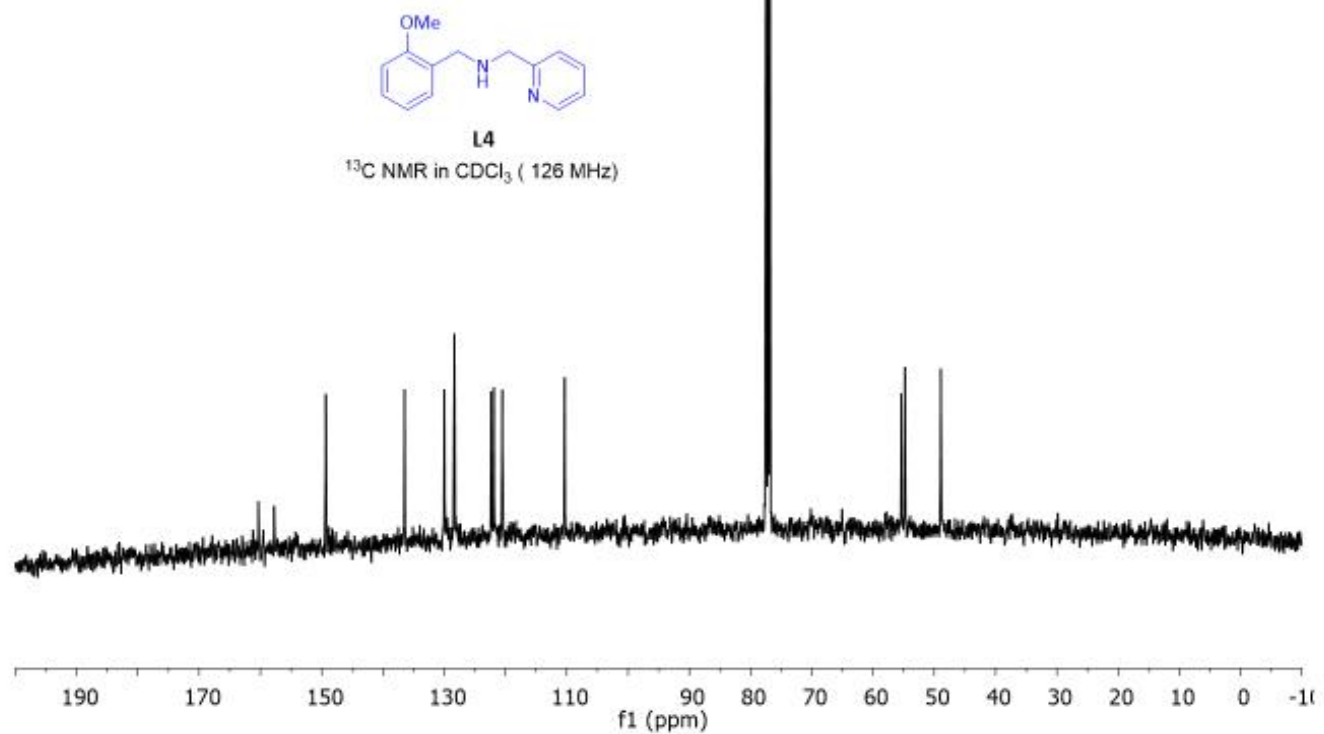

mิळ\%

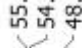




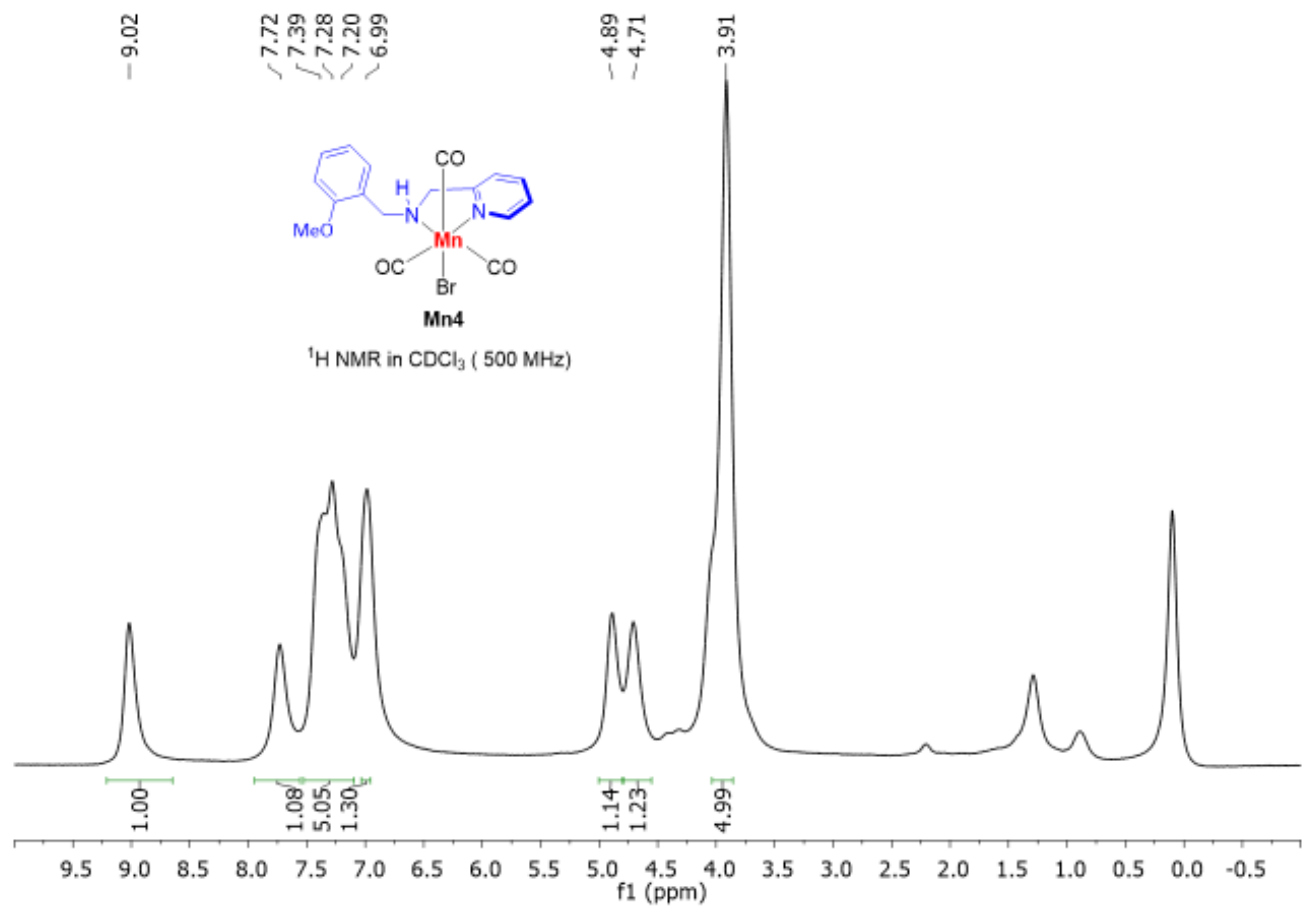

$\stackrel{\infty}{\stackrel{\sim}{*}}$

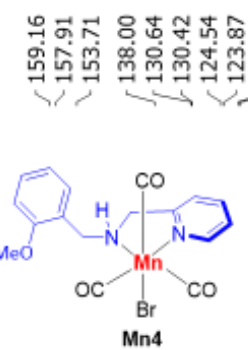

${ }^{13} \mathrm{C} \mathrm{NMR} \mathrm{in} \mathrm{CDCl}_{3}(126 \mathrm{MHz})$
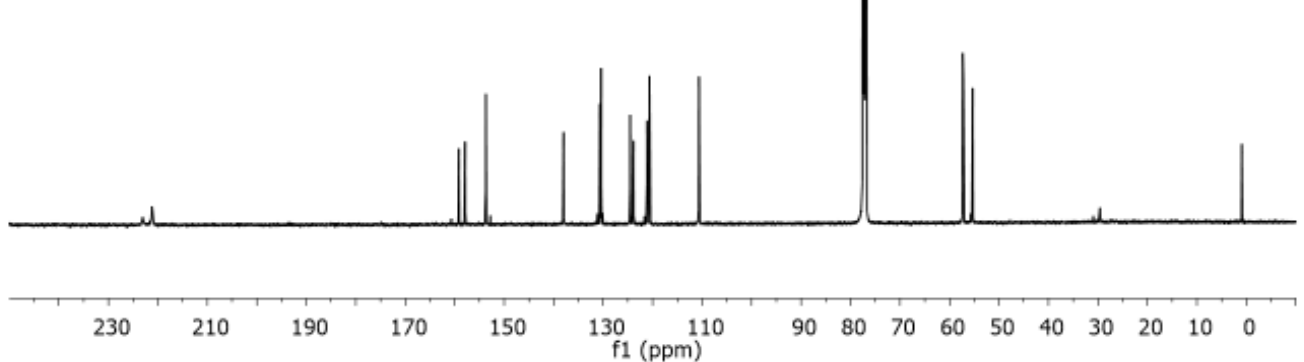

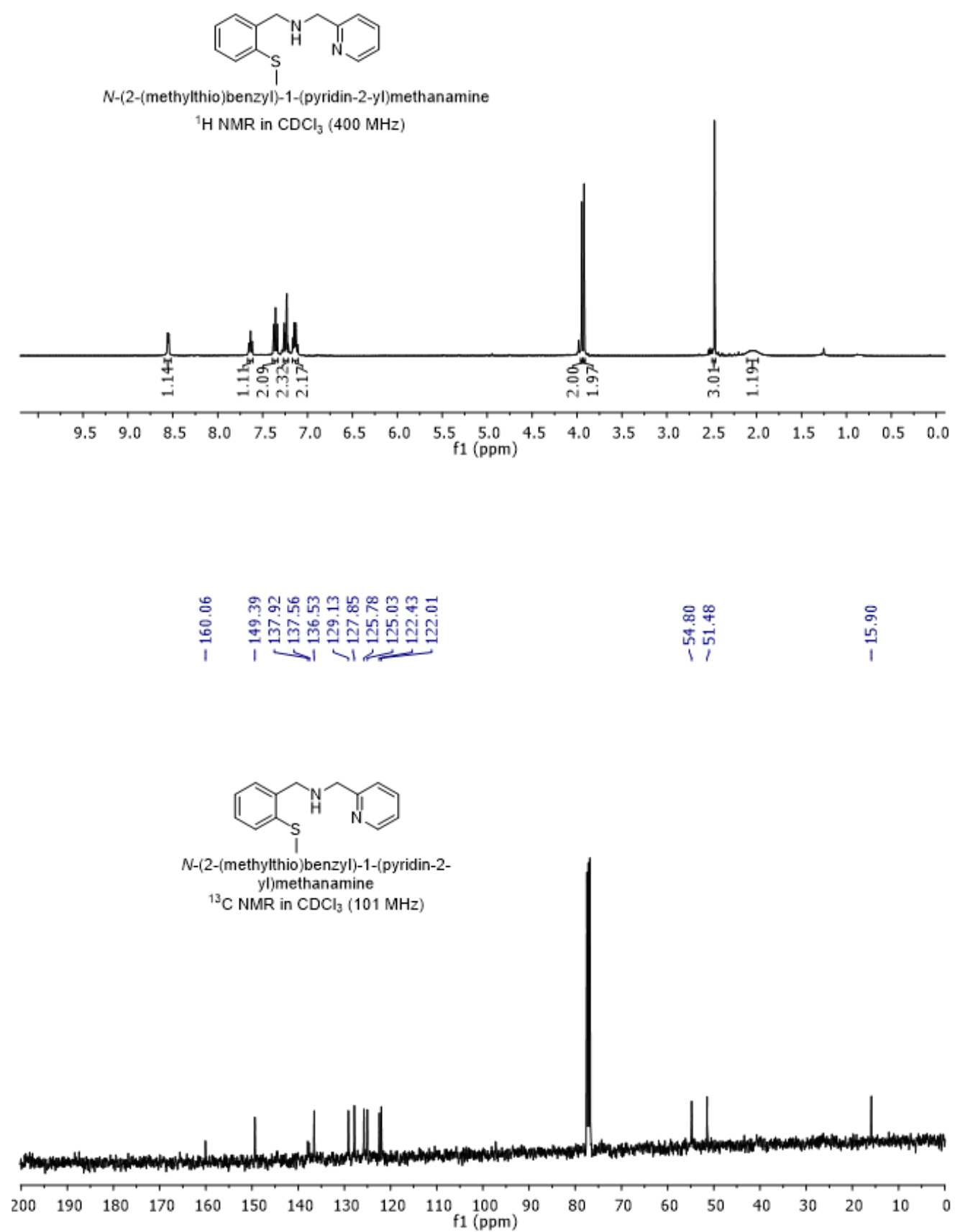


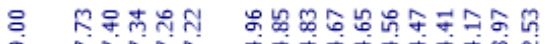

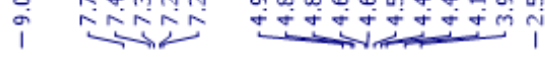

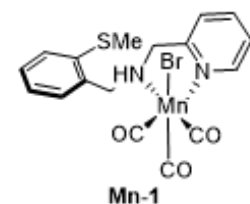

${ }^{1} \mathrm{H} \mathrm{NMR}$ in $\mathrm{CDCl}_{3}(500 \mathrm{MHz})$
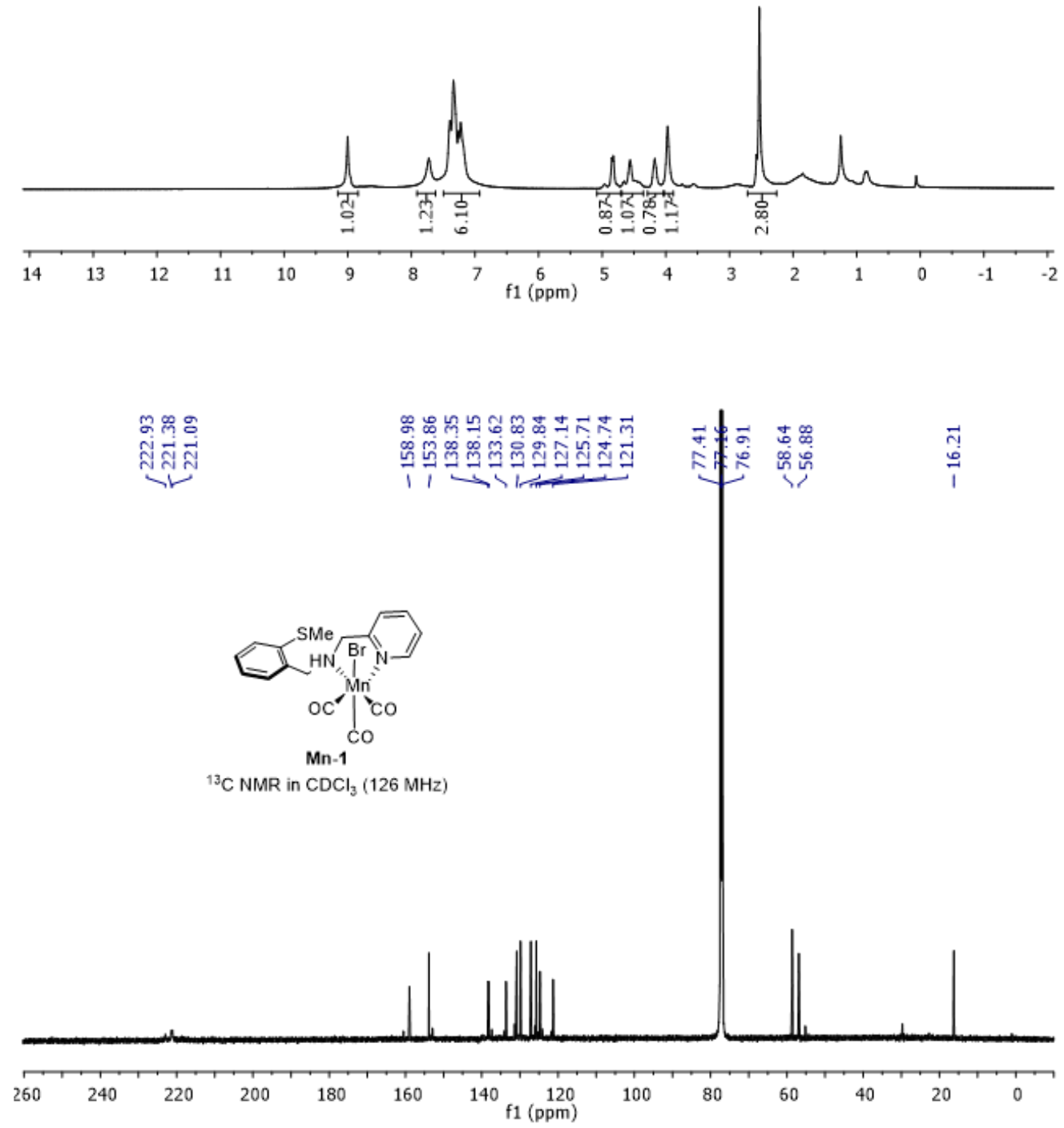


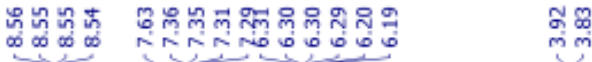

$\overbrace{\mathrm{N}} \mathrm{N} \backslash \mathrm{O}$

1-(furan-2-yl)- $N$-(pyridin-2-ylmethyl)methanamine

${ }^{1} \mathrm{H} \mathrm{NMR}$ in $\mathrm{CDCl}_{3}(500 \mathrm{MHz})$

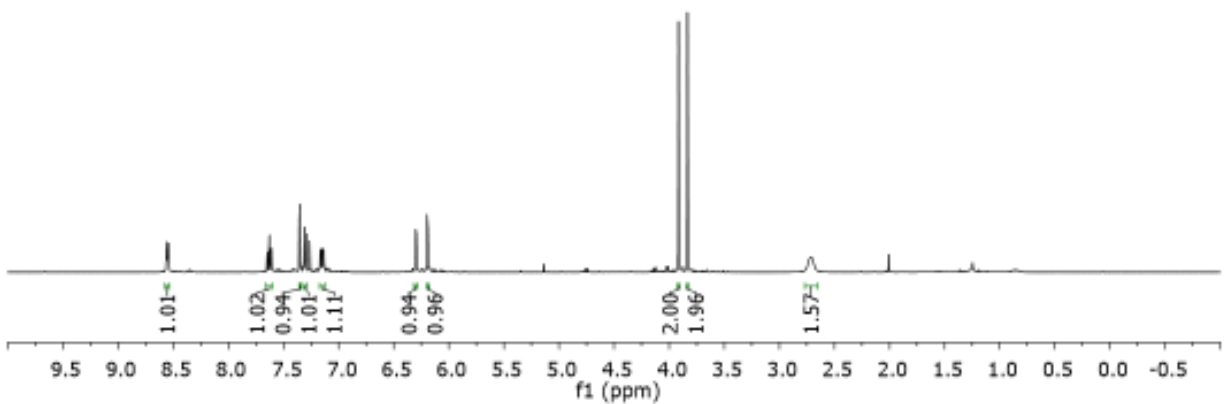

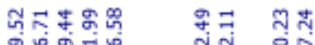

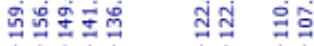

站

ㄴ,

1

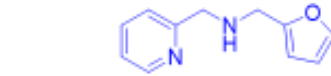

1-(furan-2-yl)-N-(pyridin-2-ylmethyl)methanamine

${ }^{13} \mathrm{C} \mathrm{NMR}$ in $\mathrm{CDCl}_{3}(126 \mathrm{MHz})$

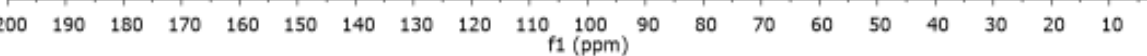




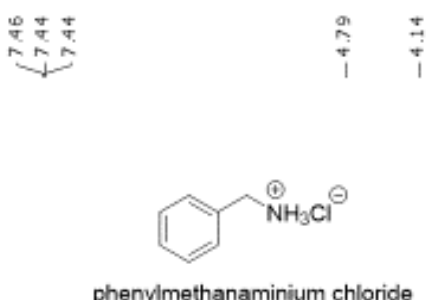

${ }^{1} \mathrm{H}$ NMR in $\mathrm{D}_{2} \mathrm{O}(400 \mathrm{MHz})$
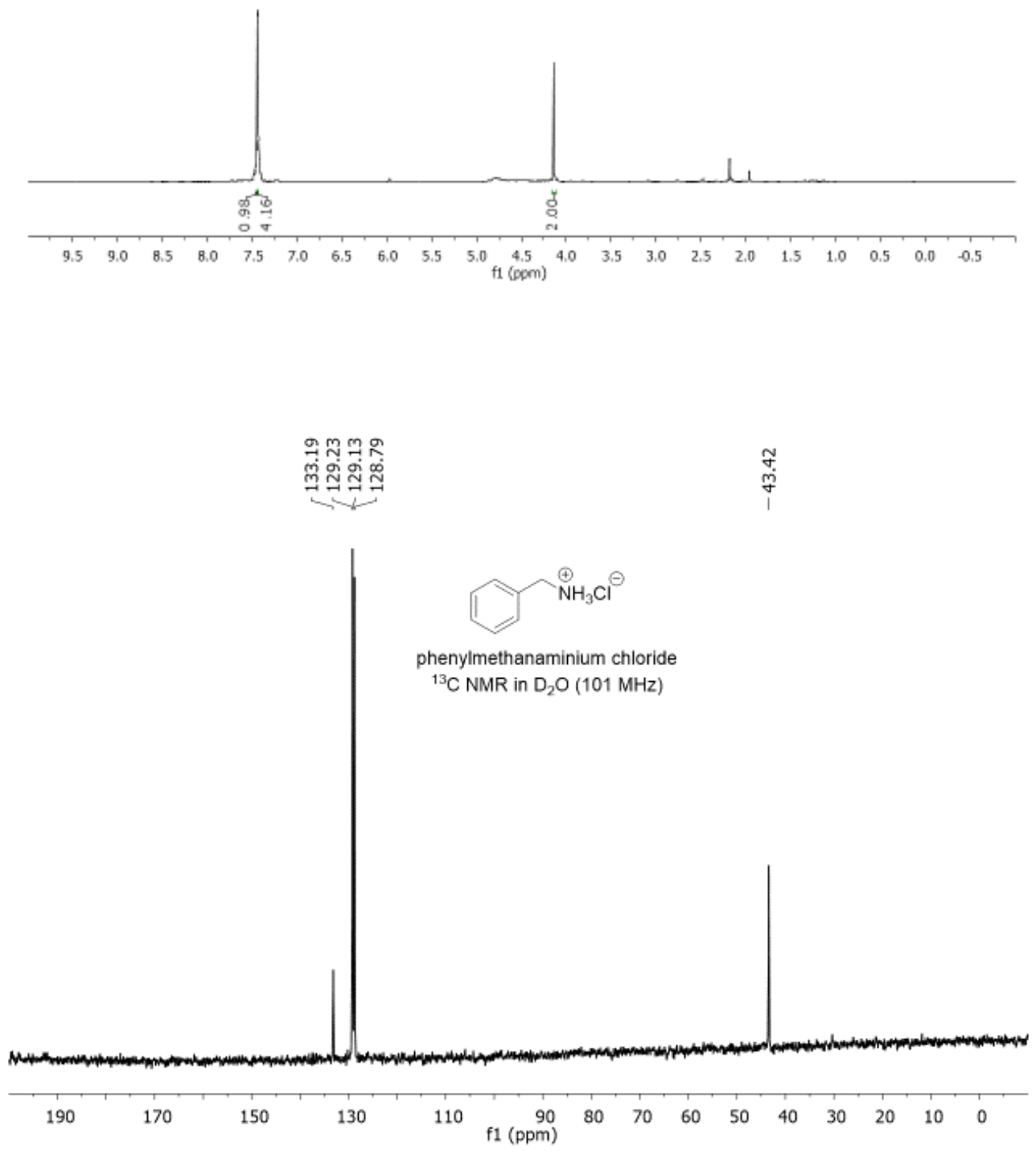

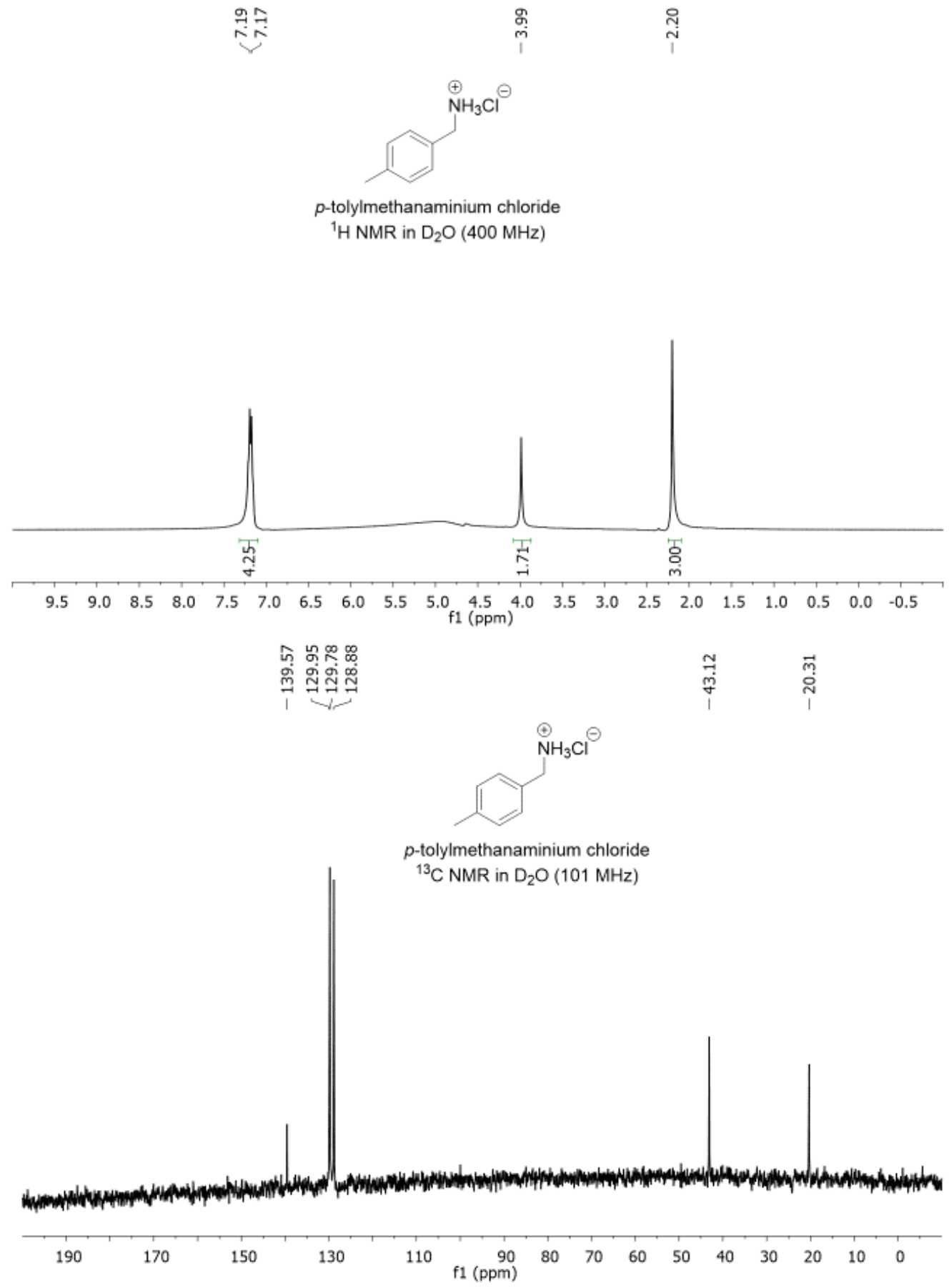


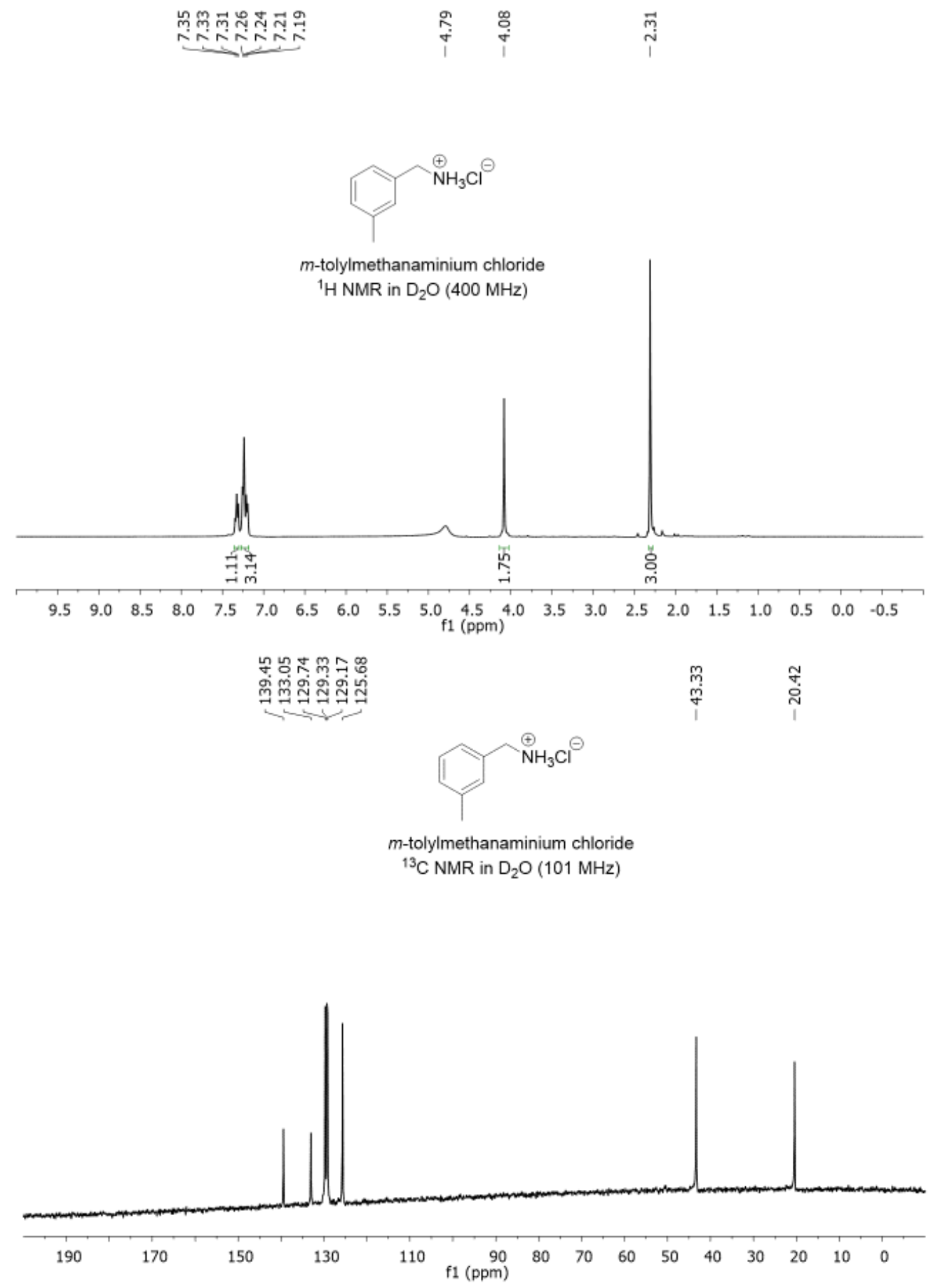



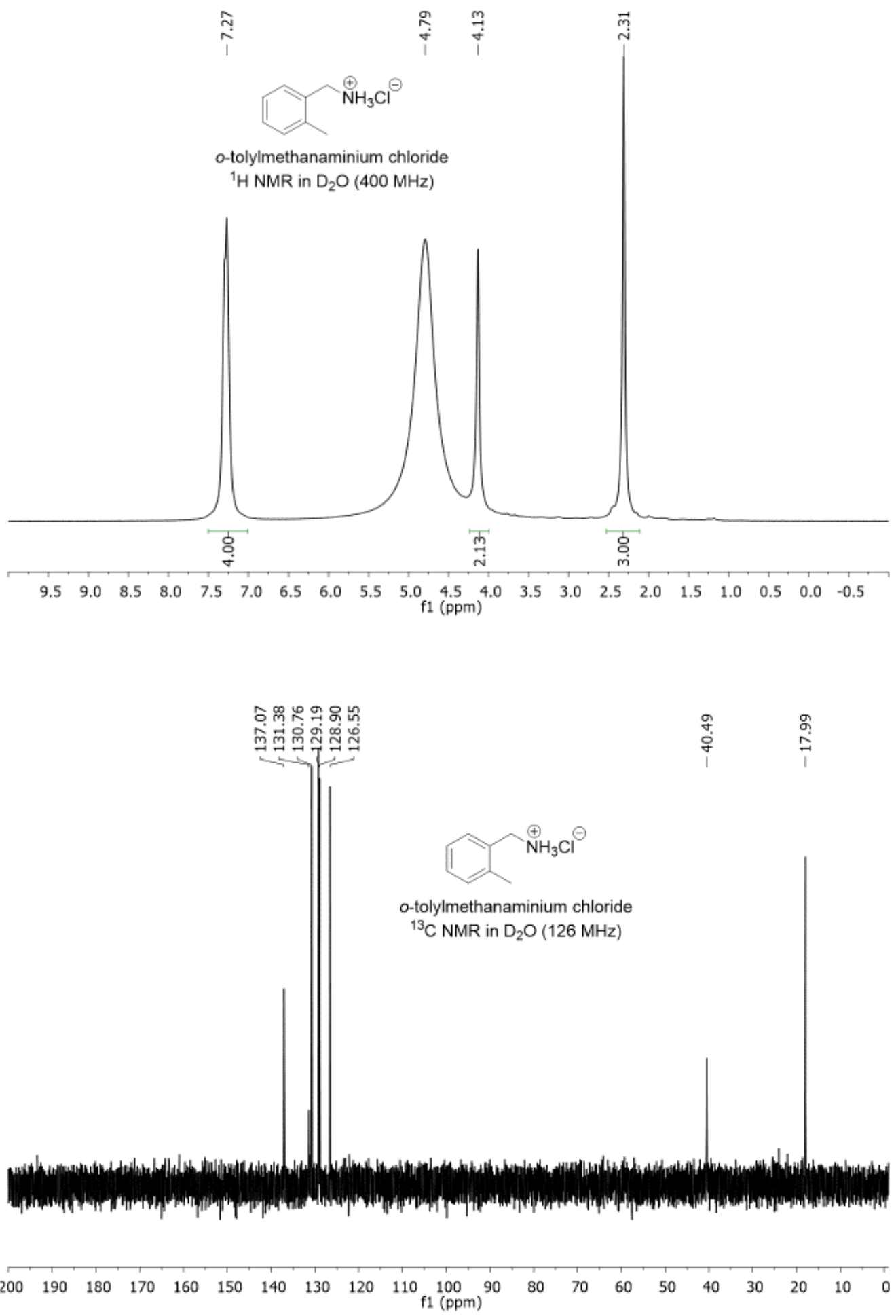


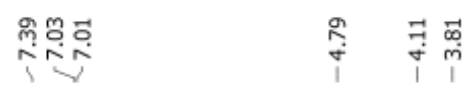

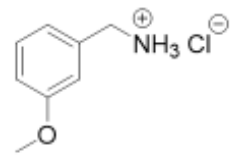

(3-methoxyphenyl)methanaminium chloride ${ }^{1} \mathrm{H}$ NMR in $\mathrm{D}_{2} \mathrm{O}(500 \mathrm{MHz})$
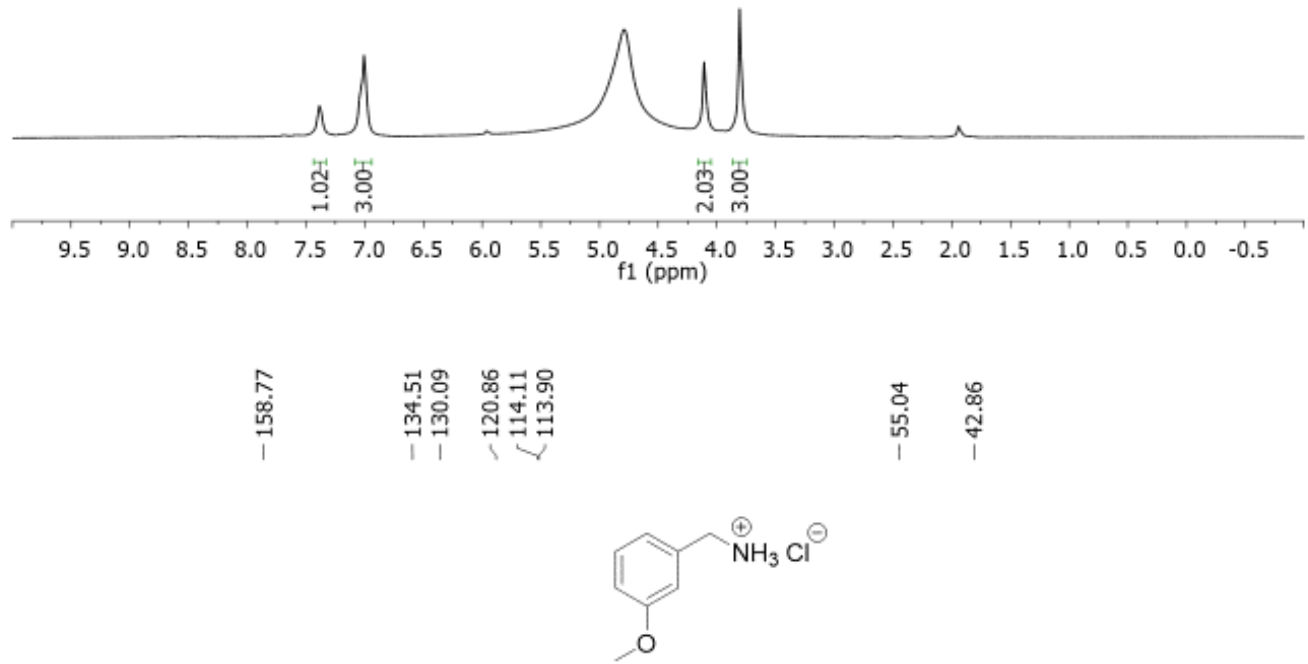

(3-methoxyphenyl)methanaminium chloride

${ }^{13} \mathrm{C}$ NMR in $\mathrm{D}_{2} \mathrm{O}(126 \mathrm{MHz})$

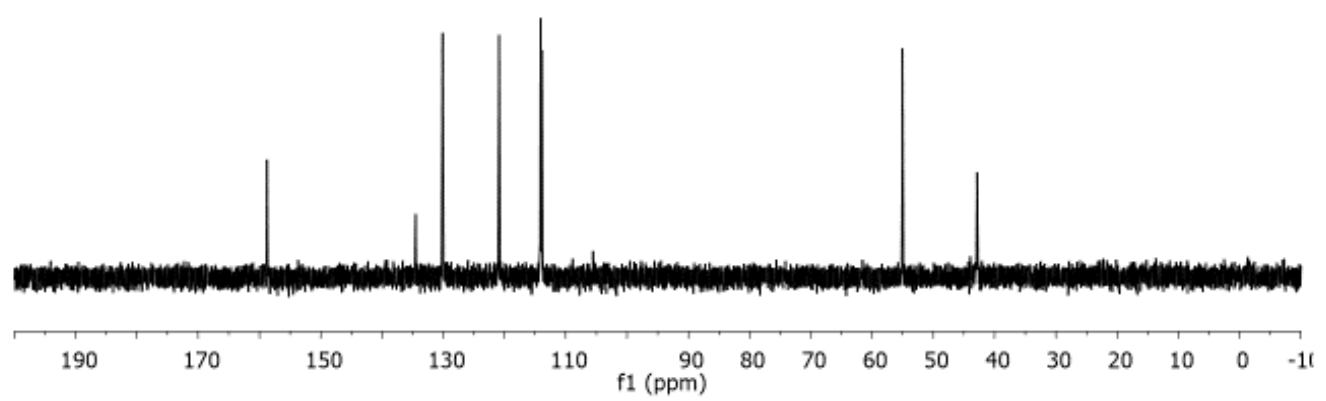




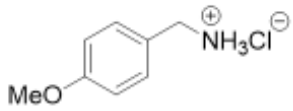

(4-methoxyphenyl)methanamine

${ }^{1} \mathrm{H} \mathrm{NMR}$ in $\mathrm{D}_{2} \mathrm{O}(400 \mathrm{MHz})$

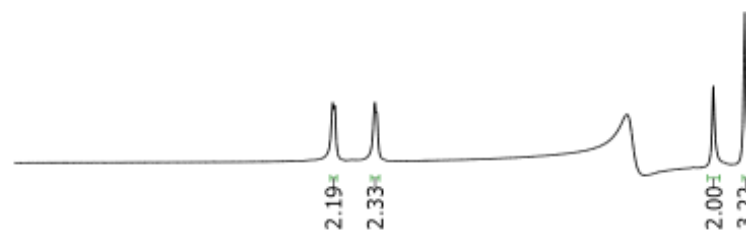

$\begin{array}{lllllllllllllllllllll}9.5 & 9.0 & 8.5 & 8.0 & 7.5 & 7.0 & 6.5 & 6.0 & 5.5 & 5.0 & 4.5 & 4.0 & 3.5 & 3.0 & 2.5 & 2.0 & 1.5 & 1.0 & 0.5 & 0.0 & -0.5\end{array}$

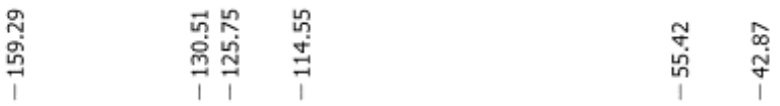

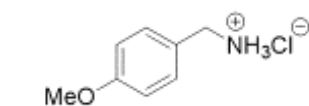

(4-methoxyphenyl)methanamine

${ }^{13} \mathrm{C}$ NMR in $\mathrm{D}_{2} \mathrm{O}(101 \mathrm{MHz})$

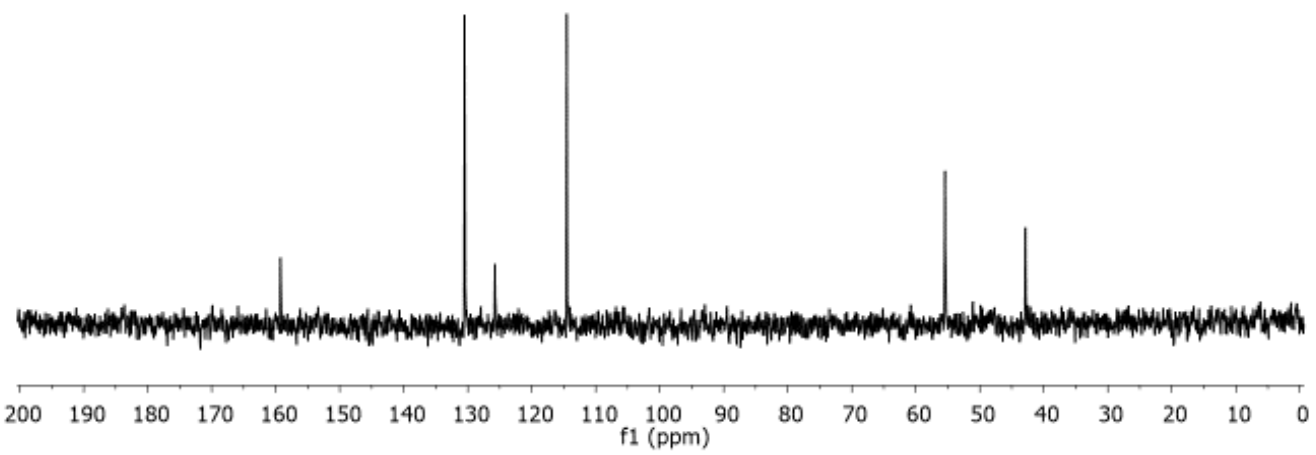




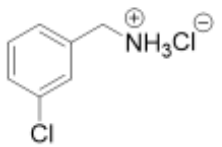

3-chlorobenzonitrile

${ }^{1} \mathrm{H} \mathrm{NMR}$ in $\mathrm{D}_{2} \mathrm{O}(400 \mathrm{MHz})$
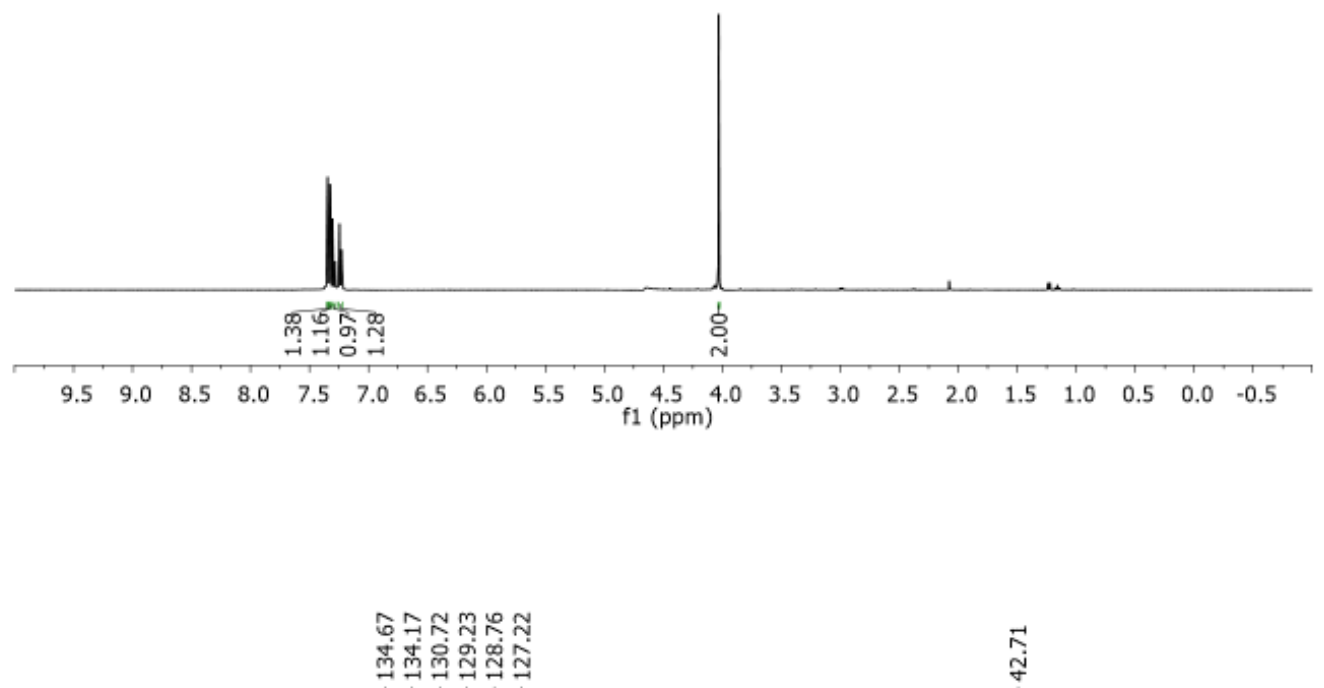

곤

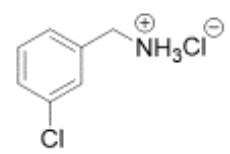

3-chlorobenzonitrile

${ }^{13} \mathrm{C}$ NMR in $\mathrm{D}_{2} \mathrm{O}(101 \mathrm{MHz})$

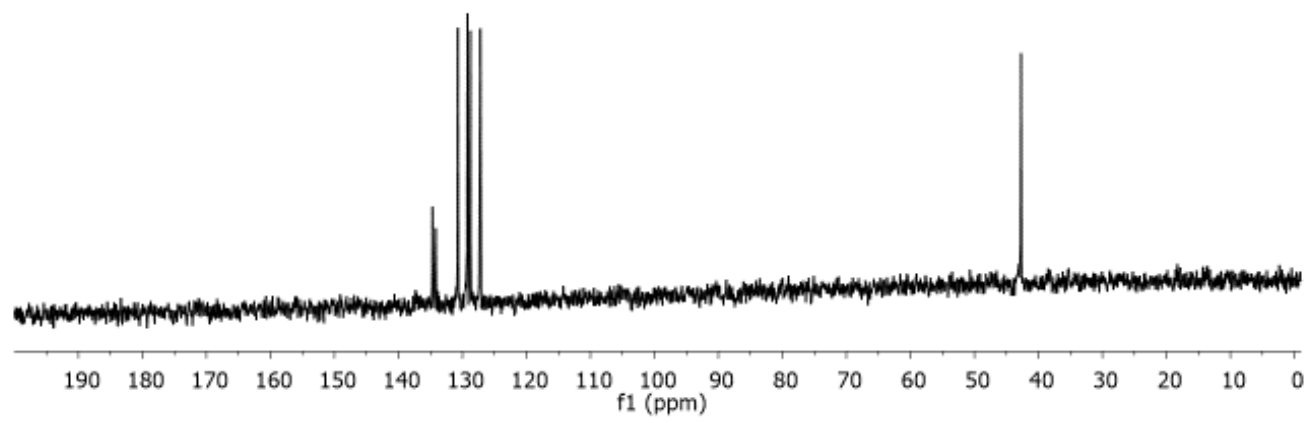




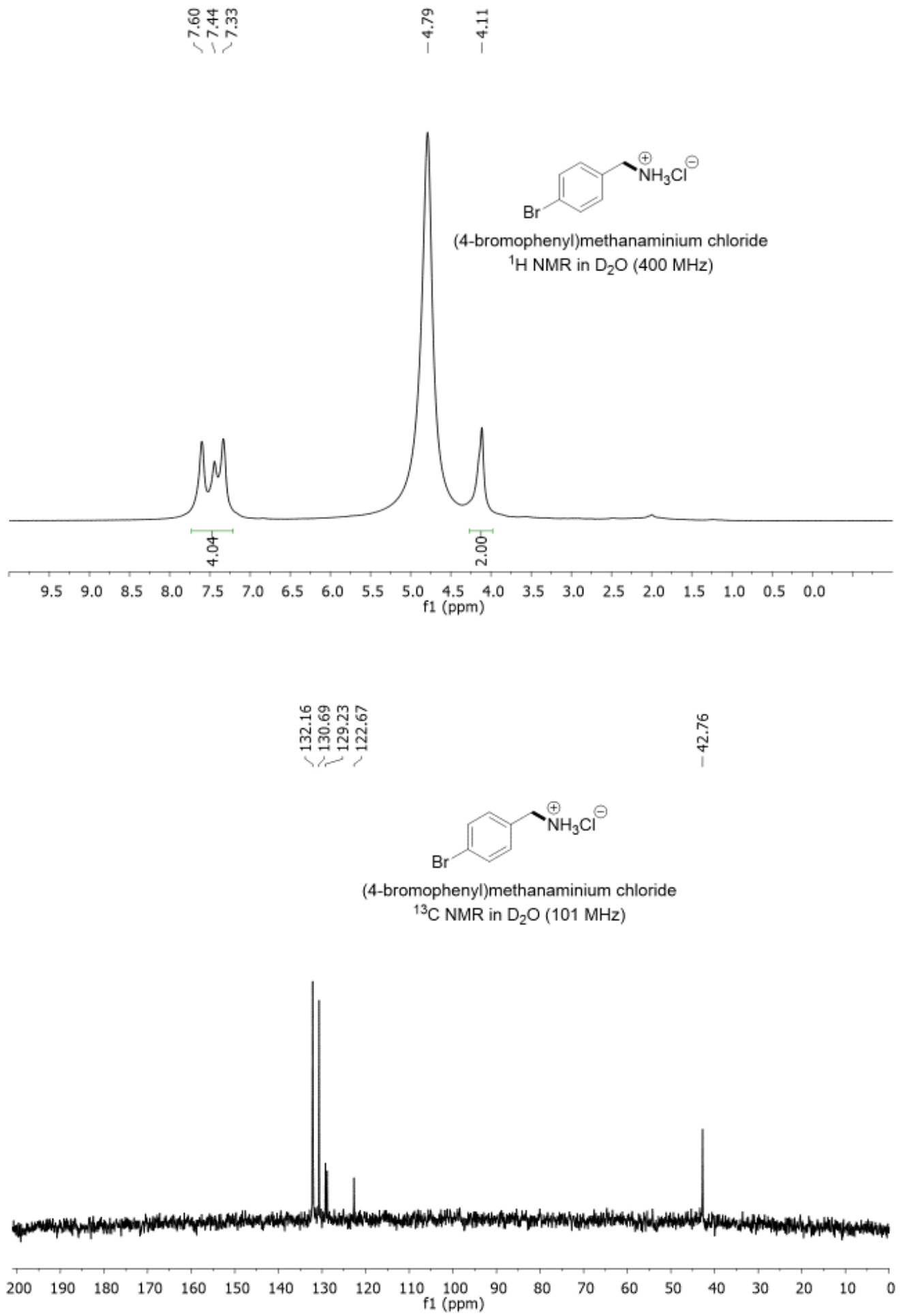




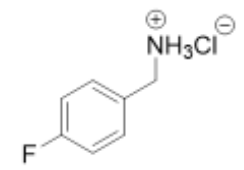

(4-fluorophenyl)methanaminium chloride ${ }^{1} \mathrm{H}$ NMR in $\mathrm{D}_{2} \mathrm{O}(400 \mathrm{MHz})$

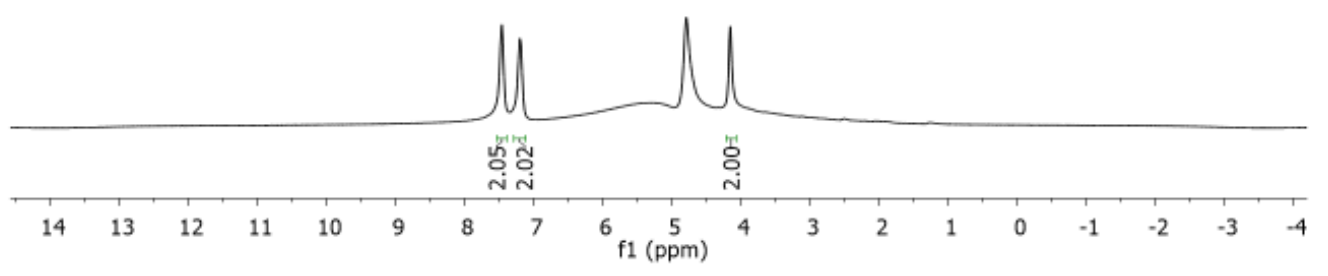

哭

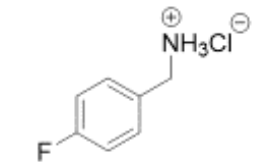

(4-fluorophenyl)methanaminium chloride

${ }^{13} \mathrm{CNMR}$ in $\mathrm{D}_{2} \mathrm{O}(101 \mathrm{MHz})$
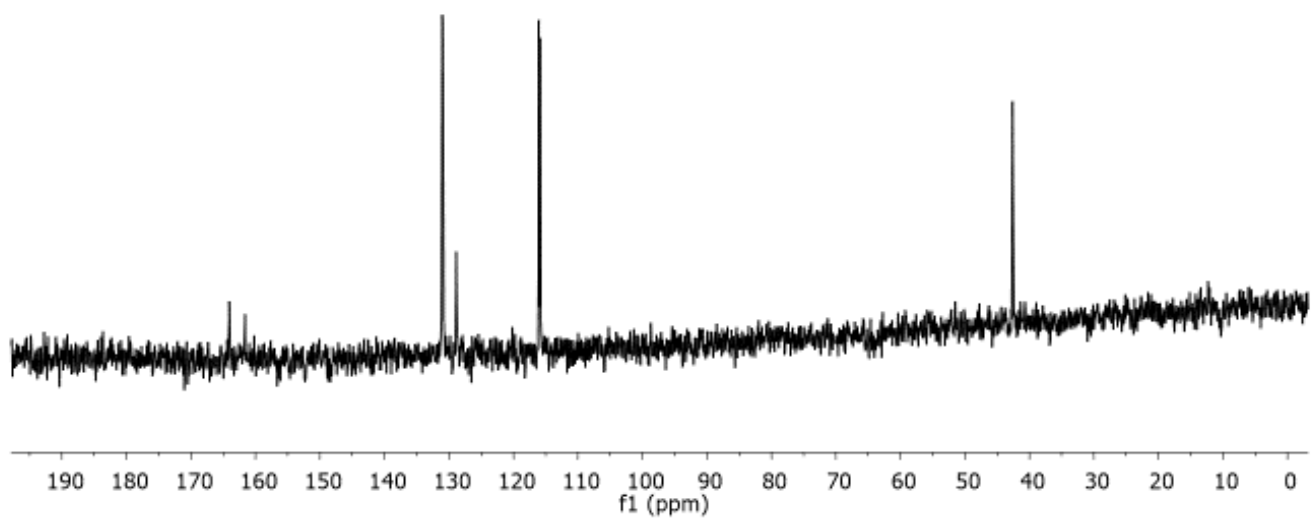


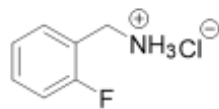

(2-fluorophenyl)methanaminium

${ }^{1} \mathrm{H}$ NMR in $\mathrm{D}_{2} \mathrm{O}(400 \mathrm{MHz})$
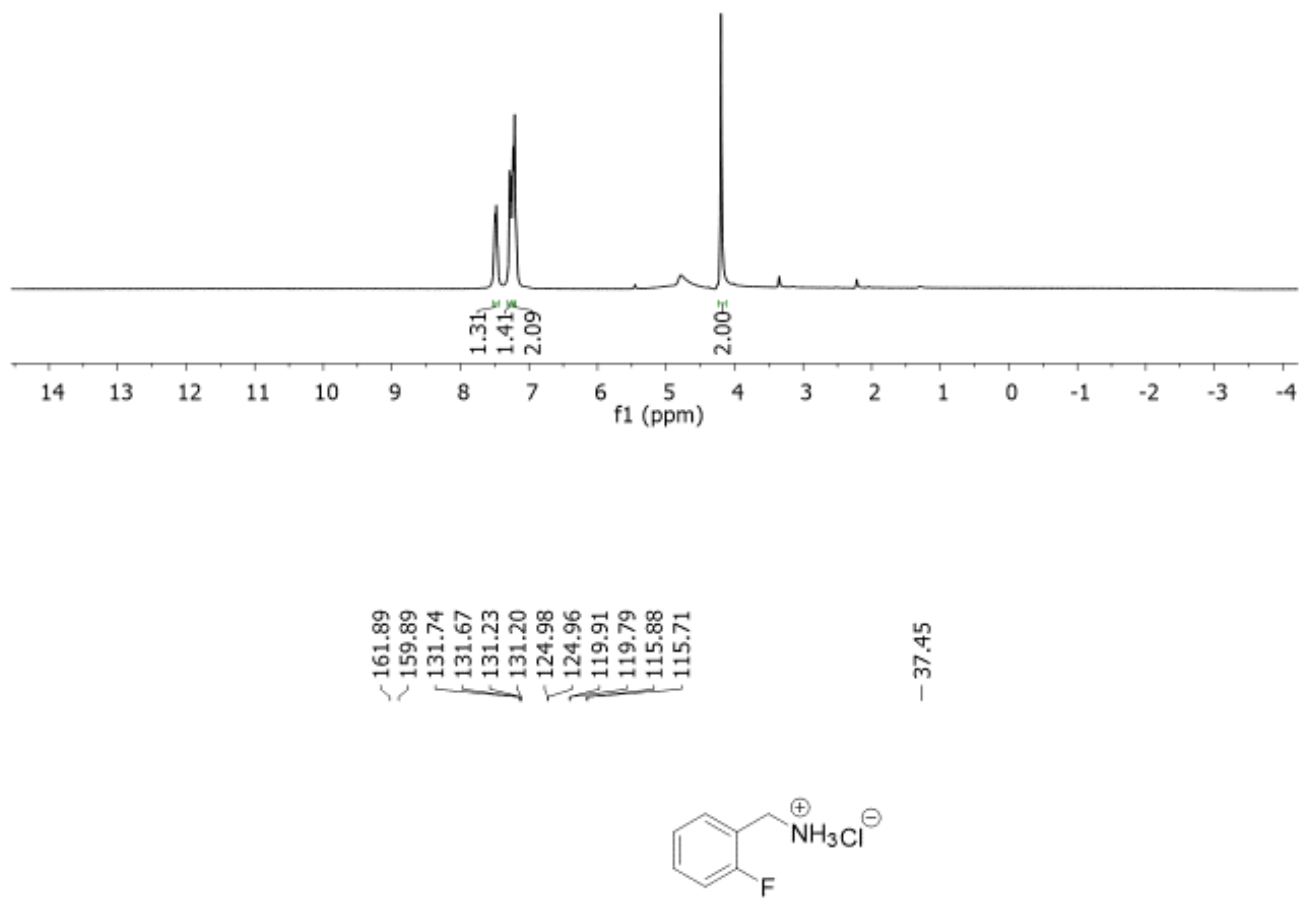

(2-fluorophenyl)methanaminium

${ }^{13} \mathrm{C}$ NMR in $\mathrm{D}_{2} \mathrm{O}(101 \mathrm{MHz})$

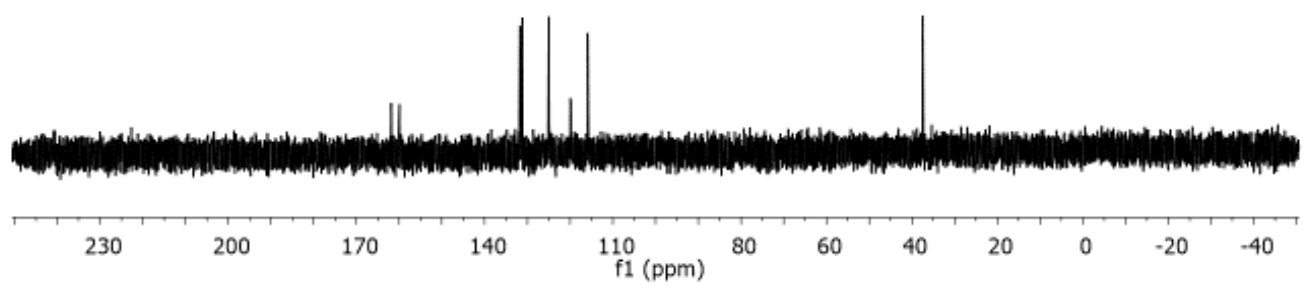




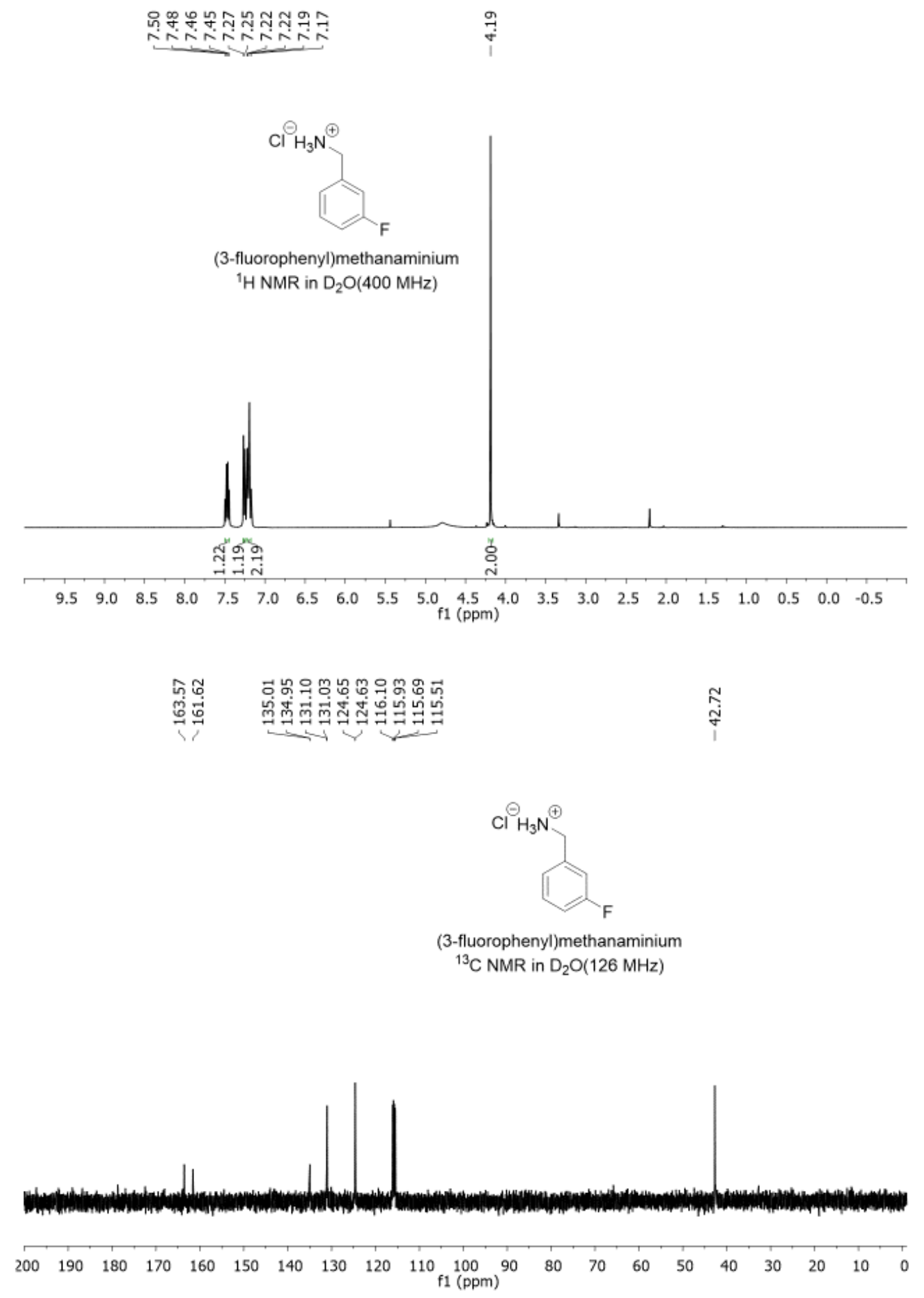




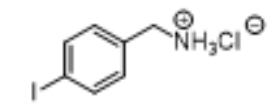

4-iodophenyl)methanaminium chloride ' $\mathrm{H} \mathrm{NMR}$ in $\mathrm{O}_{2} \mathrm{O}(400 \mathrm{MHz})$

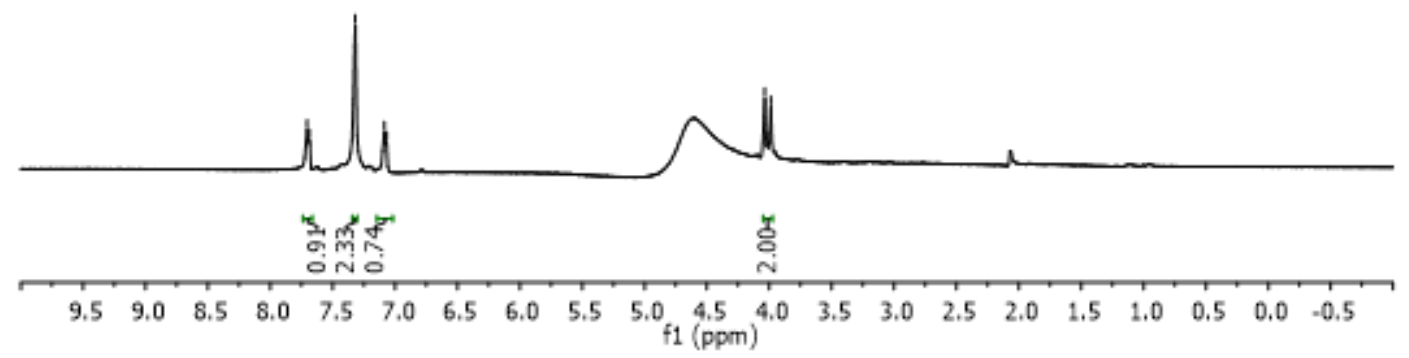

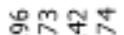

ติํํำ

$\checkmark$ is

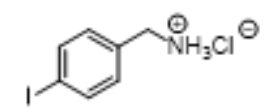

(4-iodophenyl)methanaminium chloride ${ }^{13} \mathrm{C}$ NMR in $\mathrm{D}_{2} \mathrm{O}(101 \mathrm{MHz})$

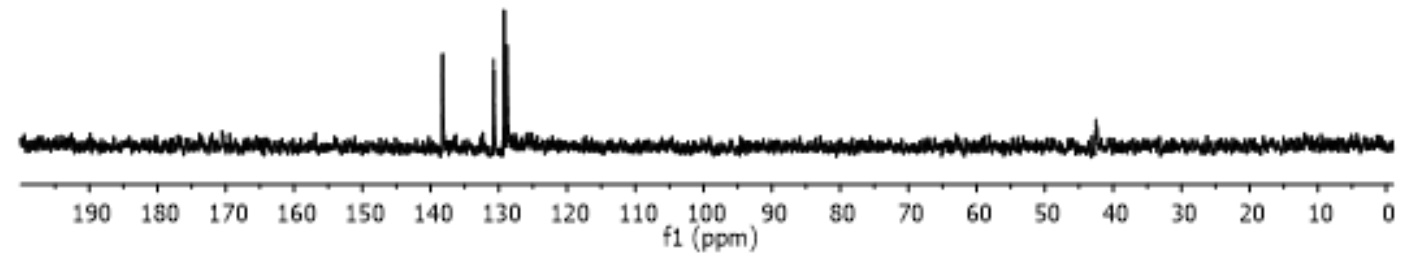




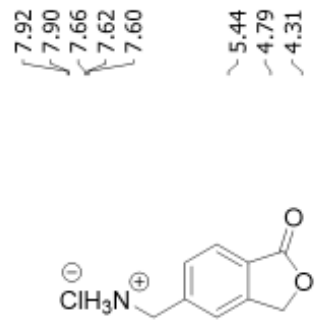

(1-oxo-1,3-dihydroisobenzofuran-5-yl)methanaminium chloride

${ }^{1} \mathrm{H}$ NMR in $\mathrm{D}_{2} \mathrm{O}(400 \mathrm{MHz})$

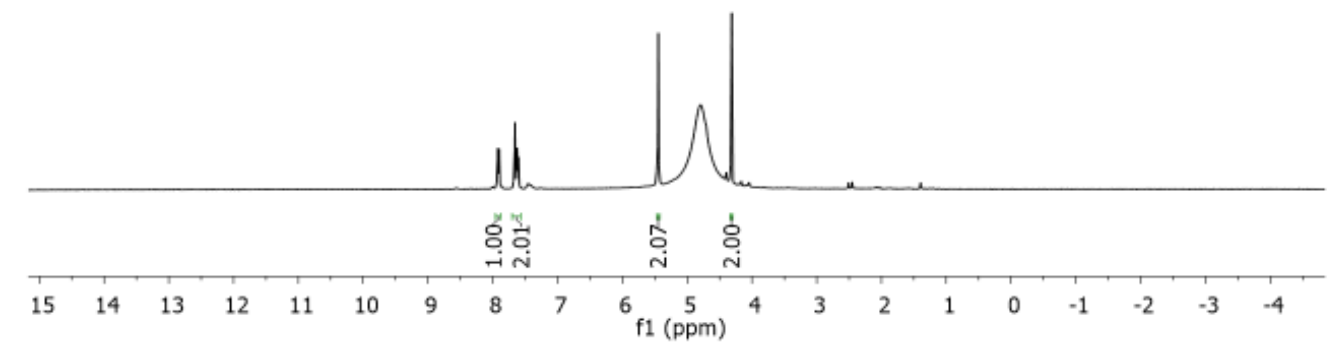

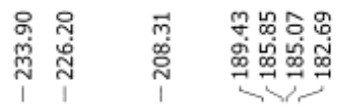

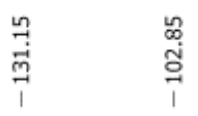

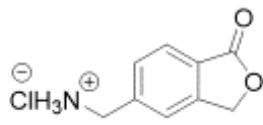

(1-oxo-1,3-dihydroisobenzofuran-5-yl)methanaminium chloride

${ }^{13} \mathrm{C} \mathrm{NMR}$ in $\mathrm{D}_{2} \mathrm{O}(126 \mathrm{MHz})$

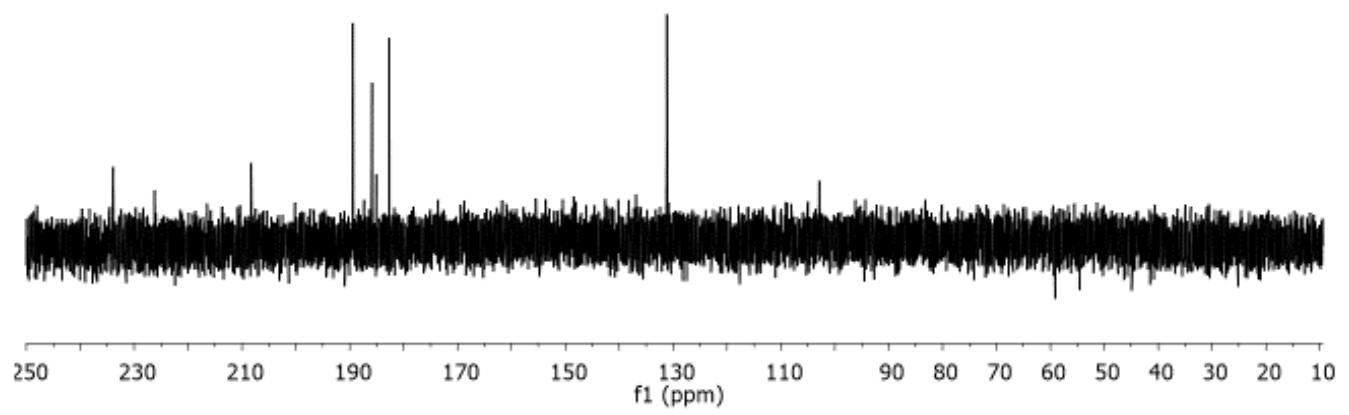




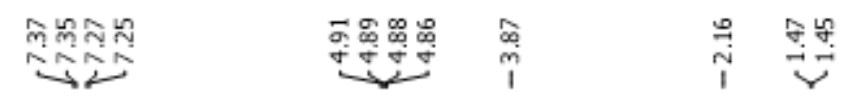

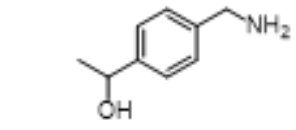

1-(4-(aminomethyl)phenyl)ethan-1-ol

$$
{ }^{1} \mathrm{H} \mathrm{NMR} \text { in } \mathrm{CDCl}_{3}(400 \mathrm{MHz})
$$
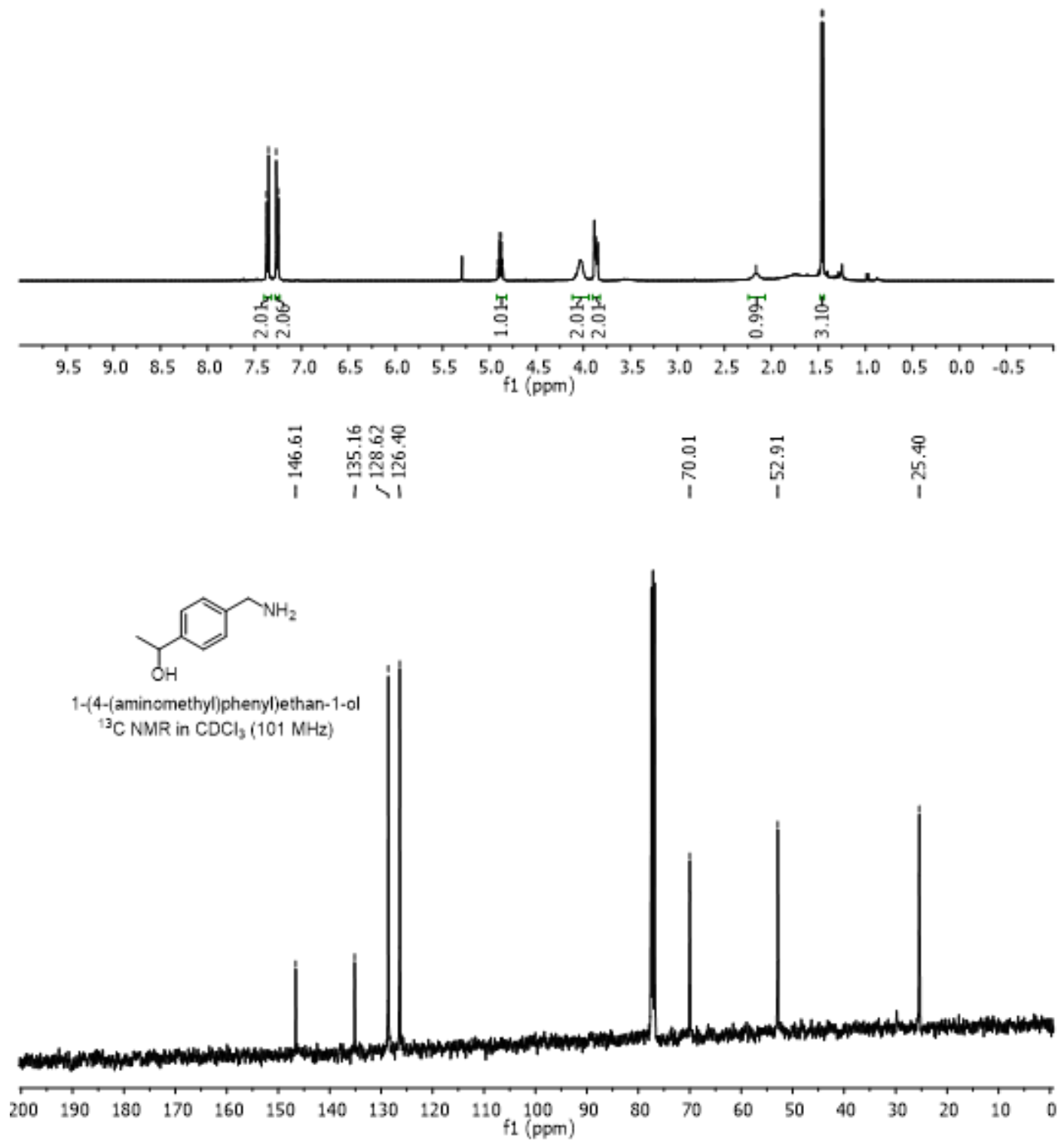


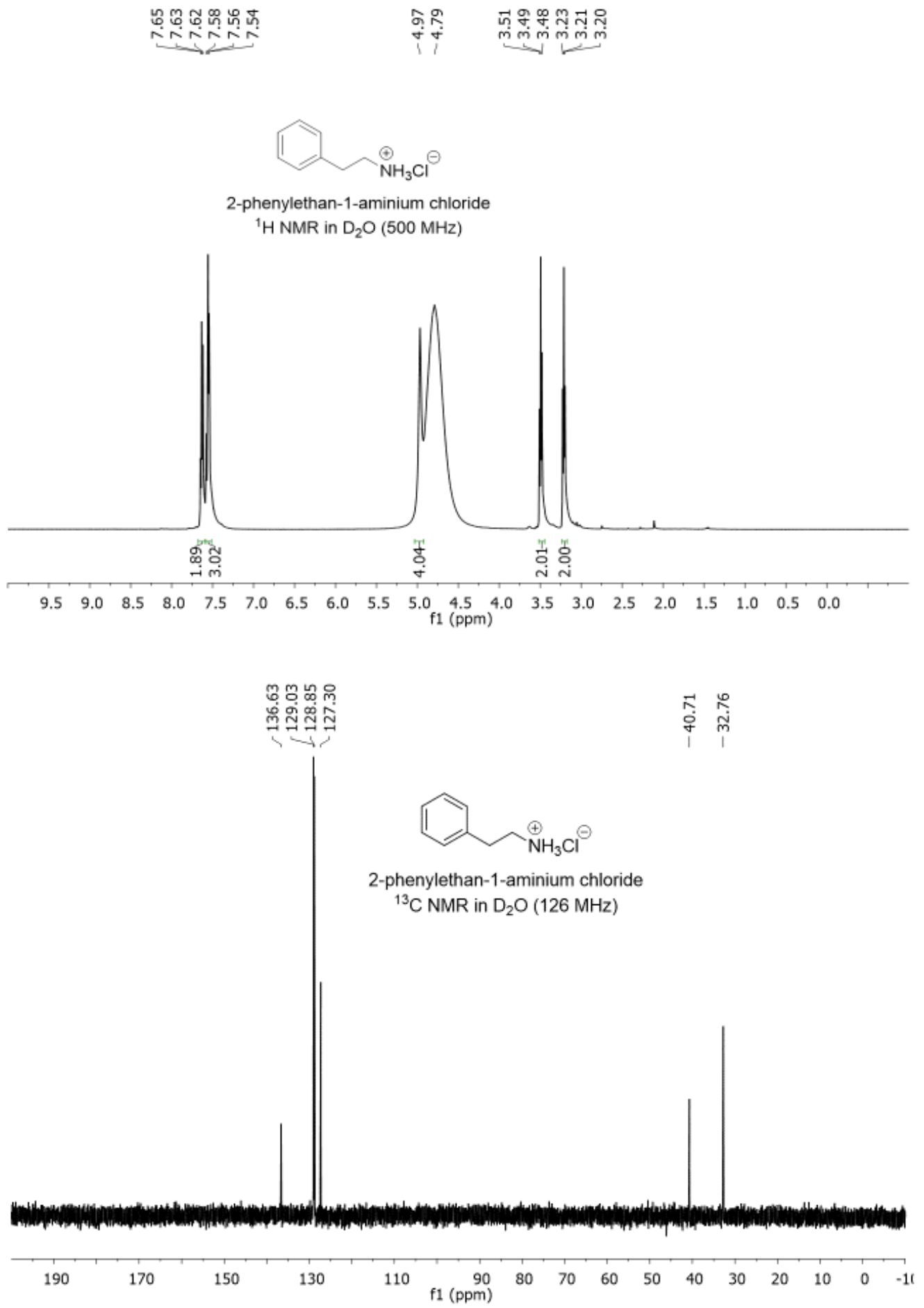



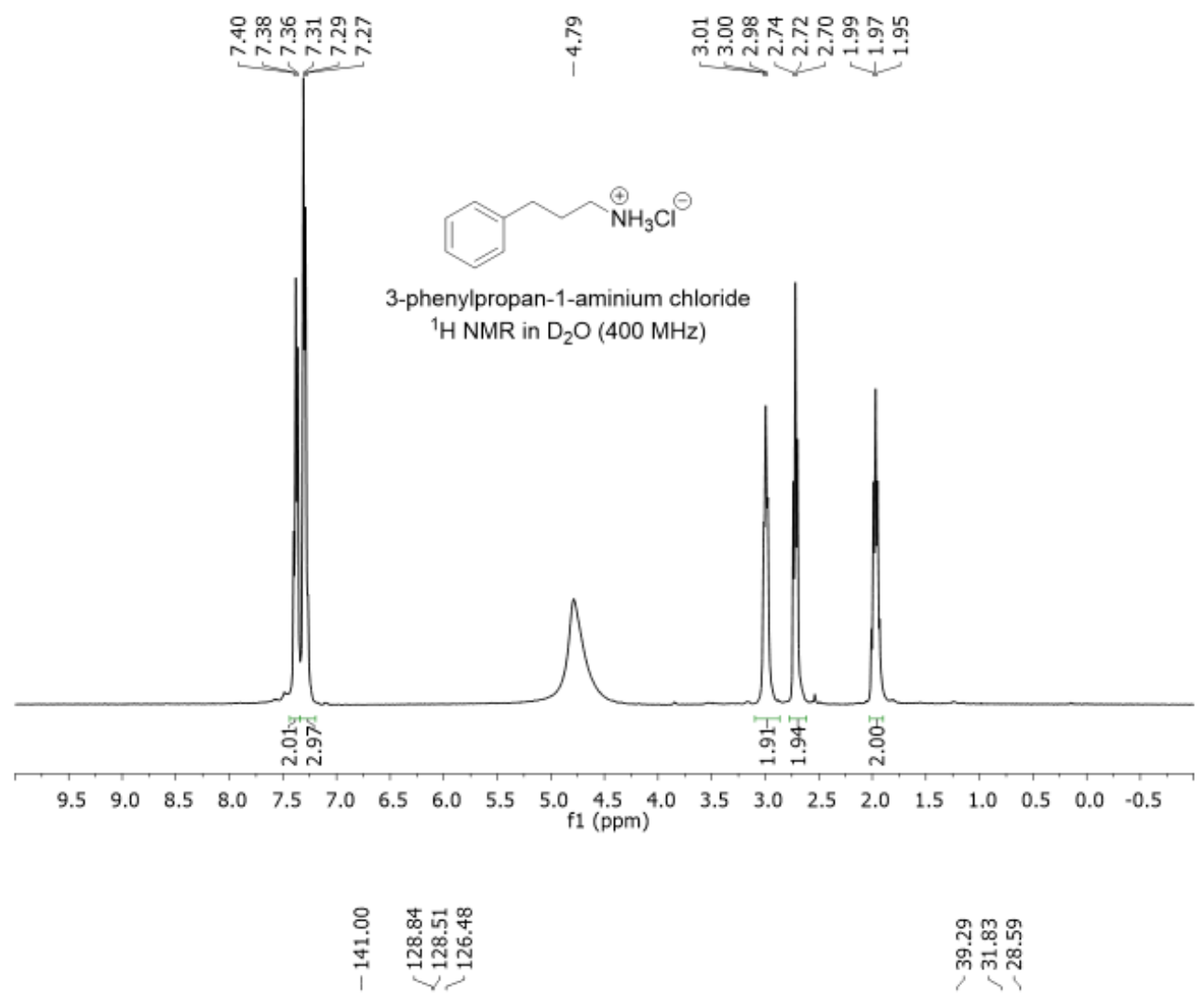

กุต ฒุ

लำ लె

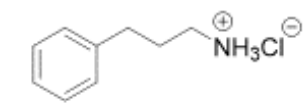

3-phenylpropan-1-aminium chloride

${ }^{13} \mathrm{C}$ NMR in $\mathrm{D}_{2} \mathrm{O}(101 \mathrm{MHz})$

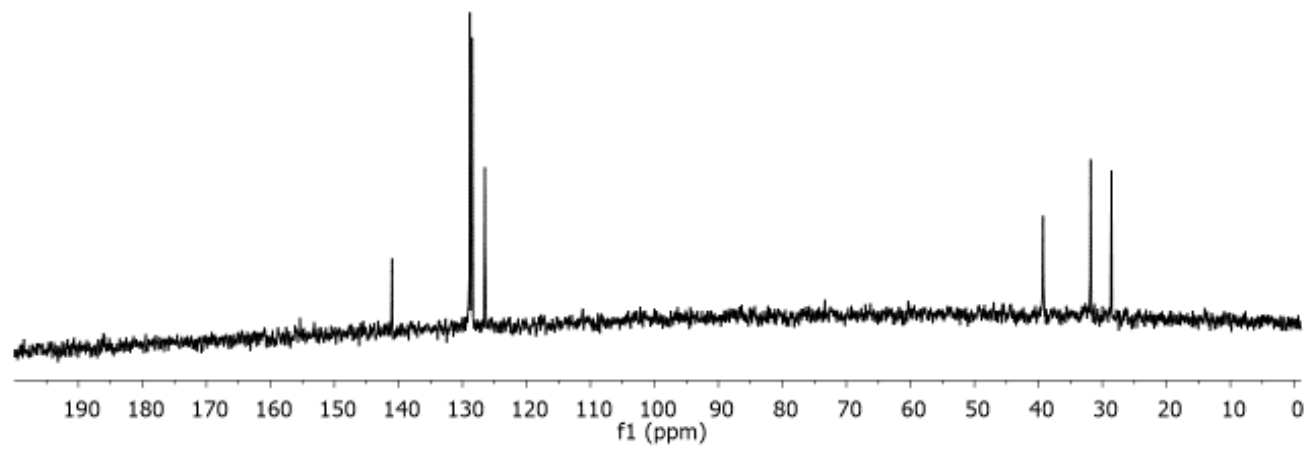


i

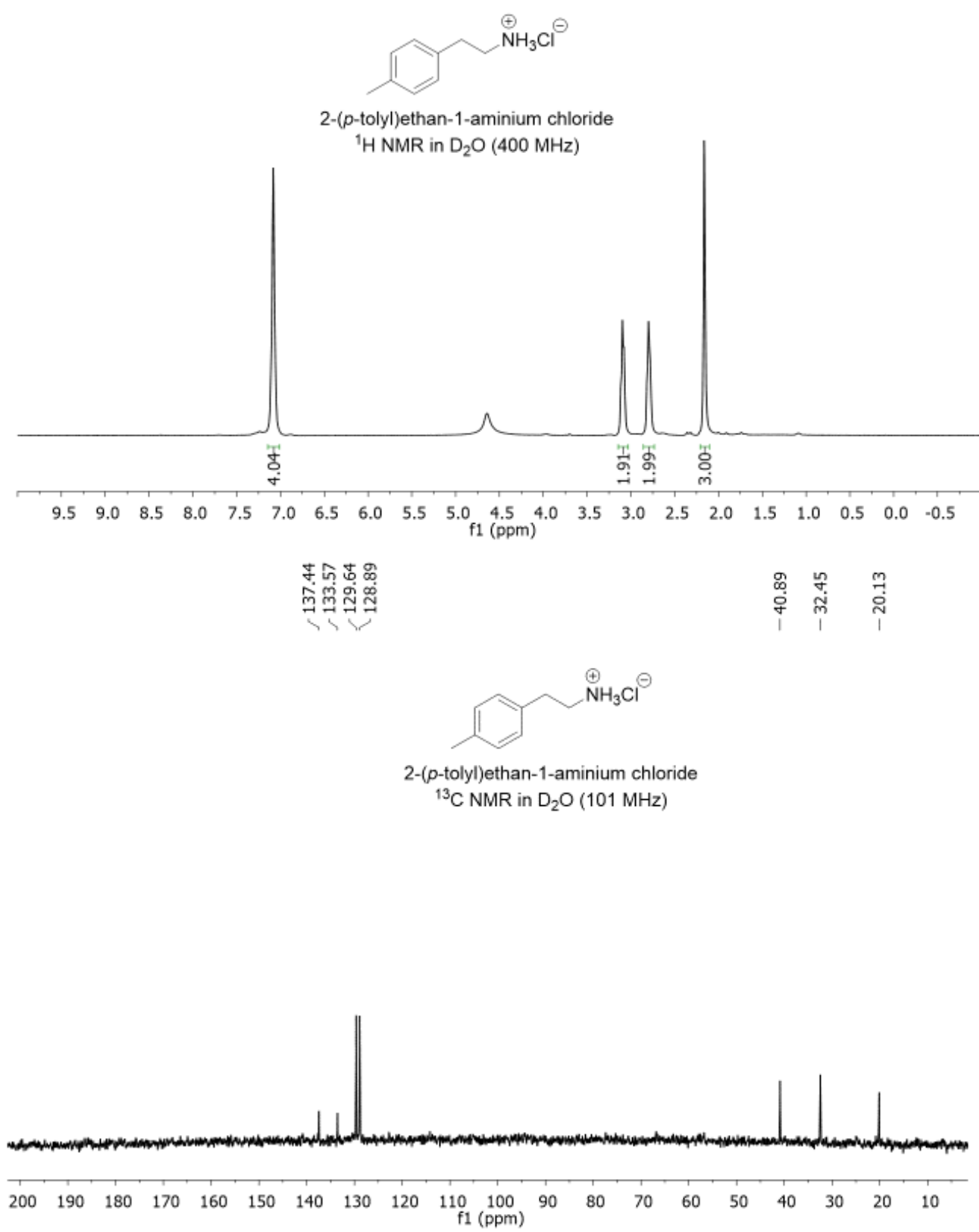



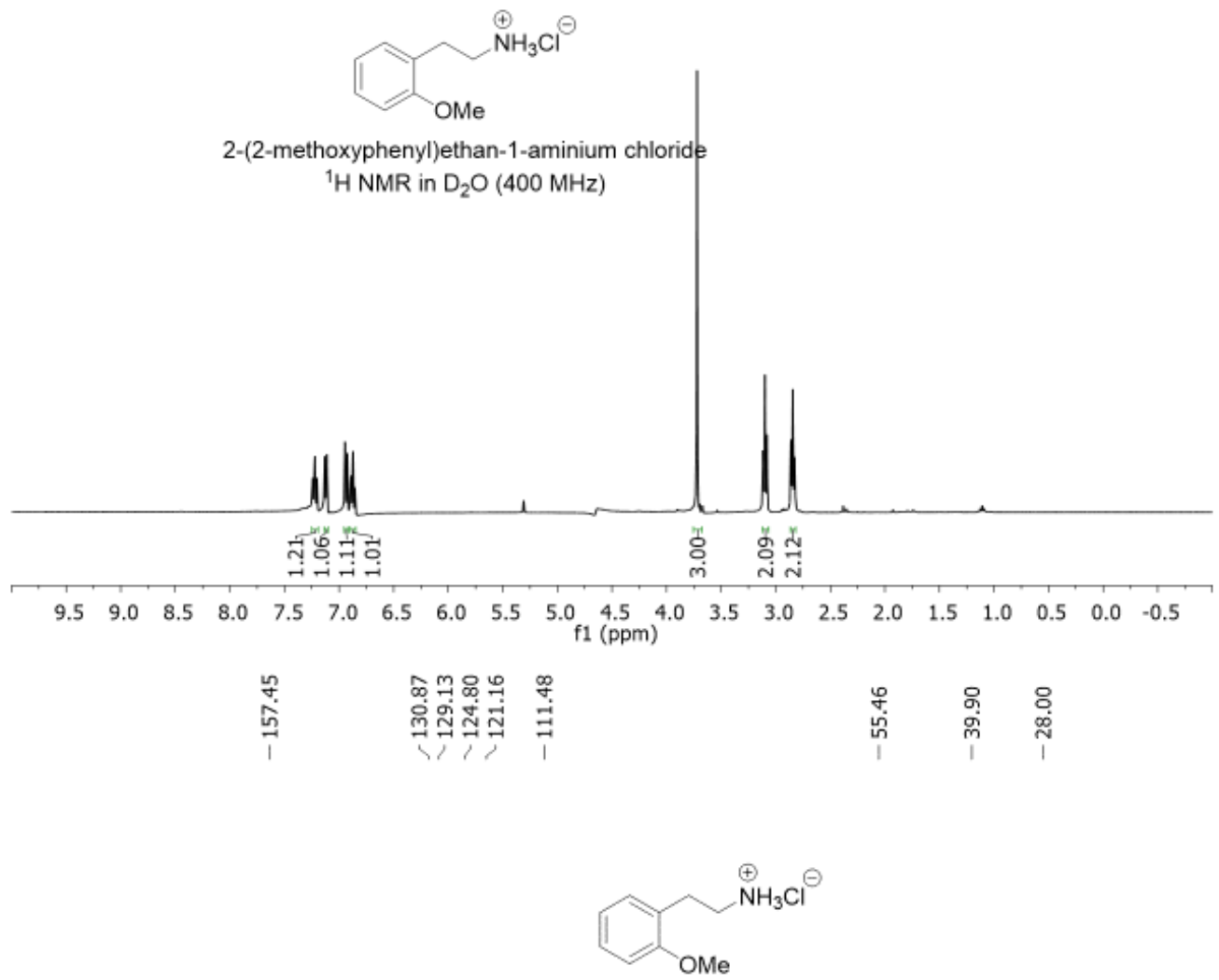

2-(2-methoxyphenyl)ethan-1-aminium chloride ${ }^{13} \mathrm{C}$ NMR in $\mathrm{D}_{2} \mathrm{O}(101 \mathrm{MHz})$

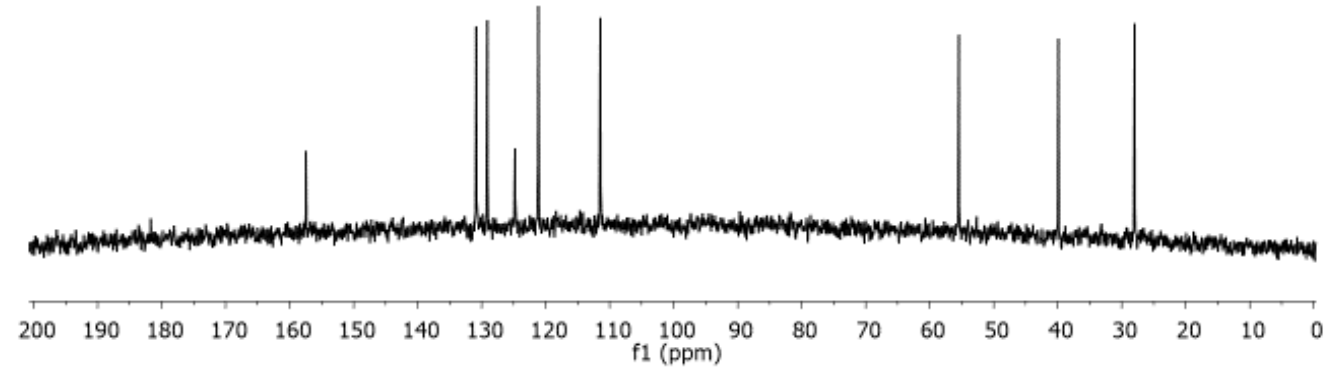




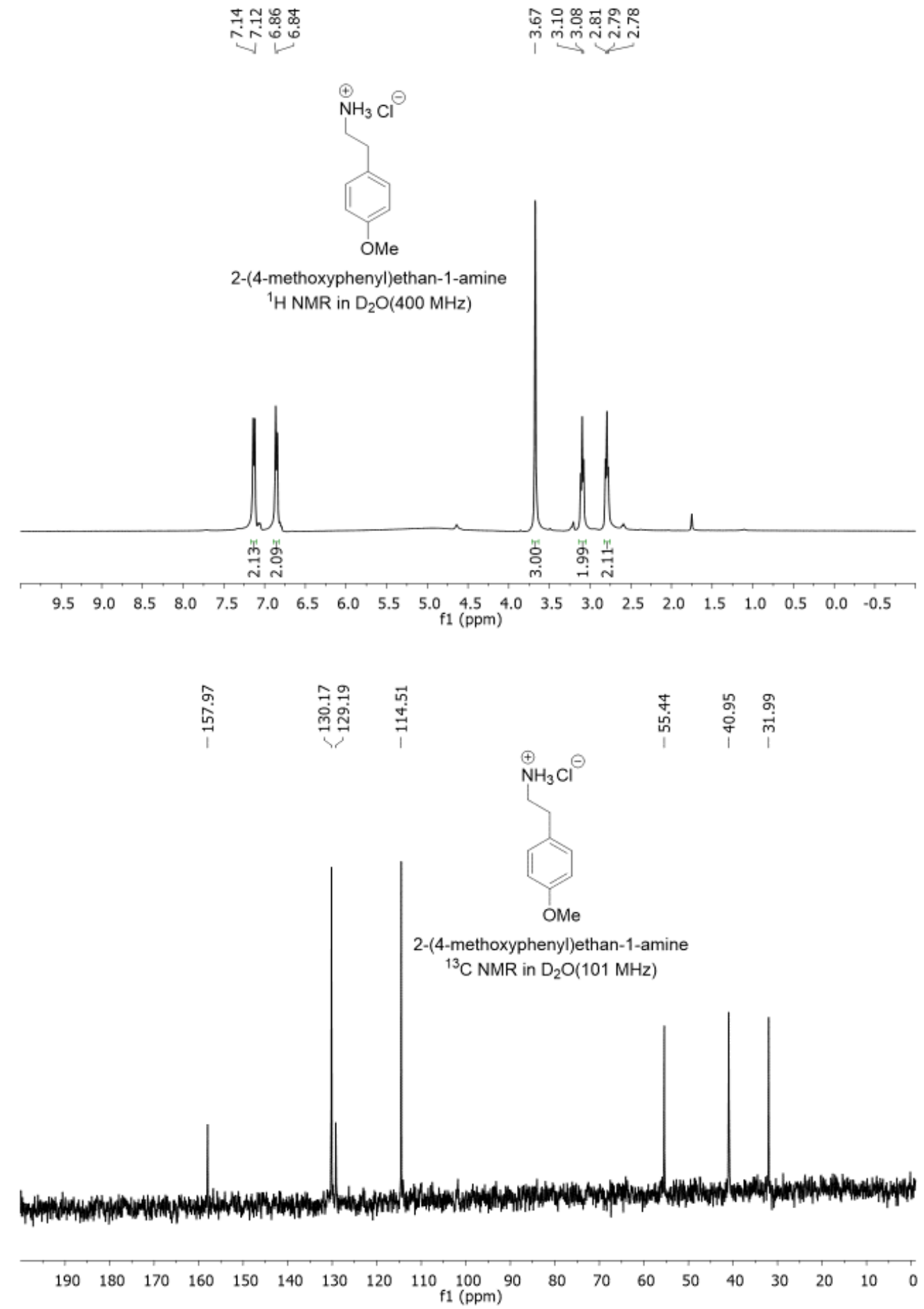




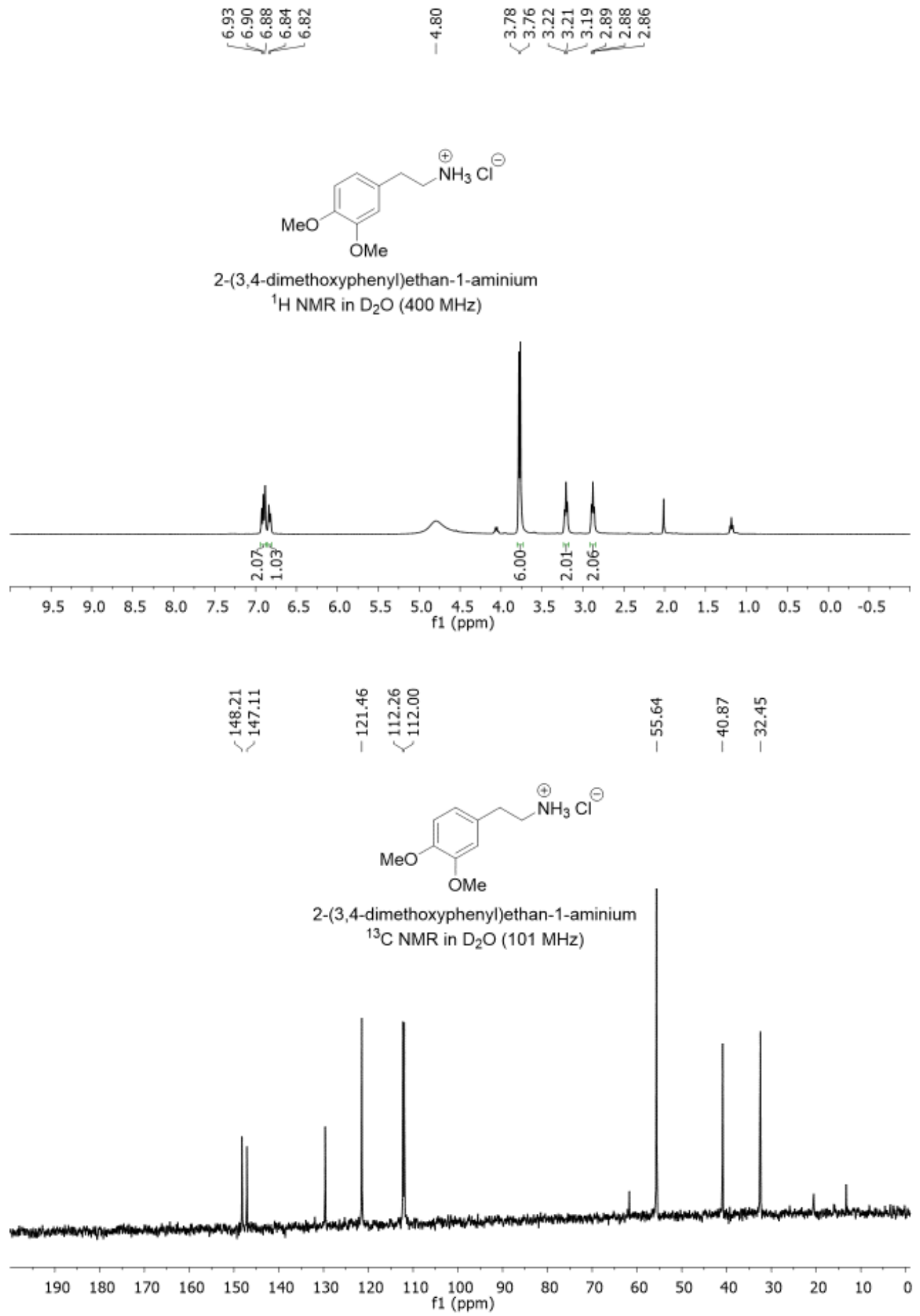



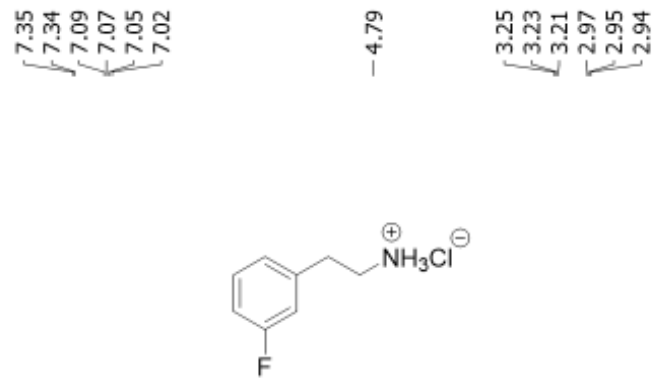

2-(3-fluorophenyl)ethan-1-aminium chloride ${ }^{1} \mathrm{H} \mathrm{NMR}$ in $\mathrm{D}_{2} \mathrm{O}(400 \mathrm{MHz})$
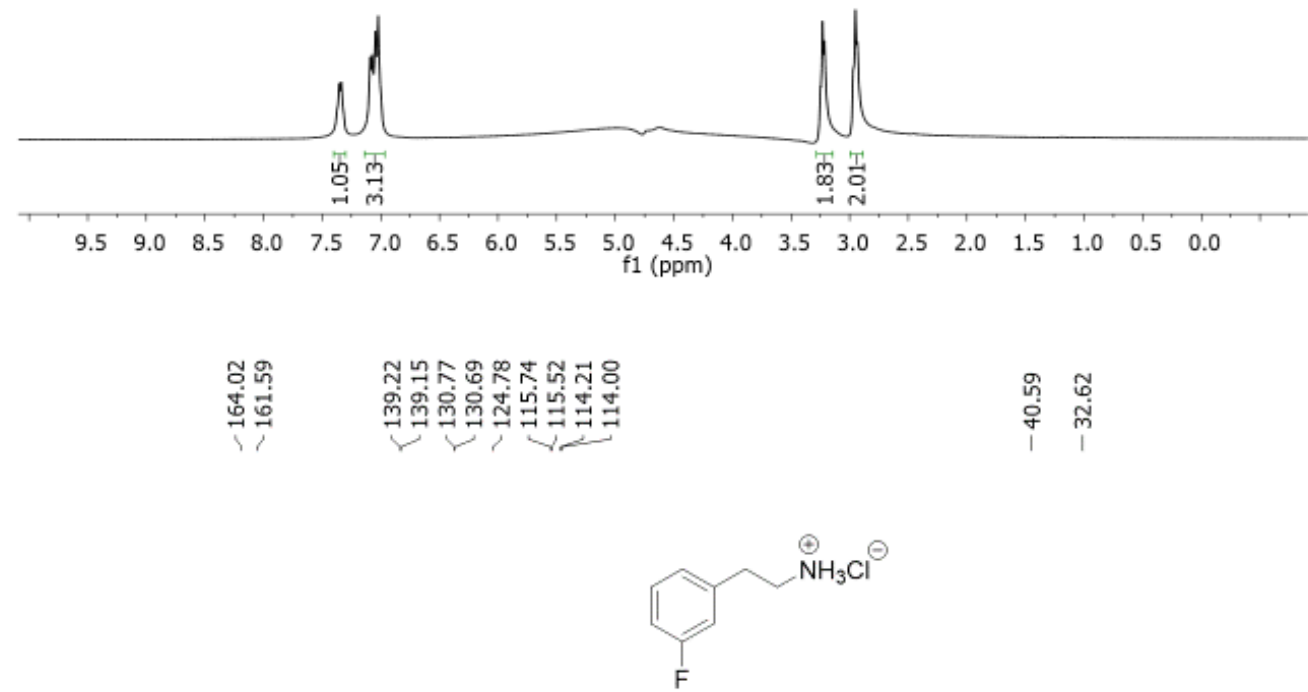

2-(3-fluorophenyl)ethan-1-aminium chloride ${ }^{13} \mathrm{C}$ NMR in $\mathrm{D}_{2} \mathrm{O}(101 \mathrm{MHz})$

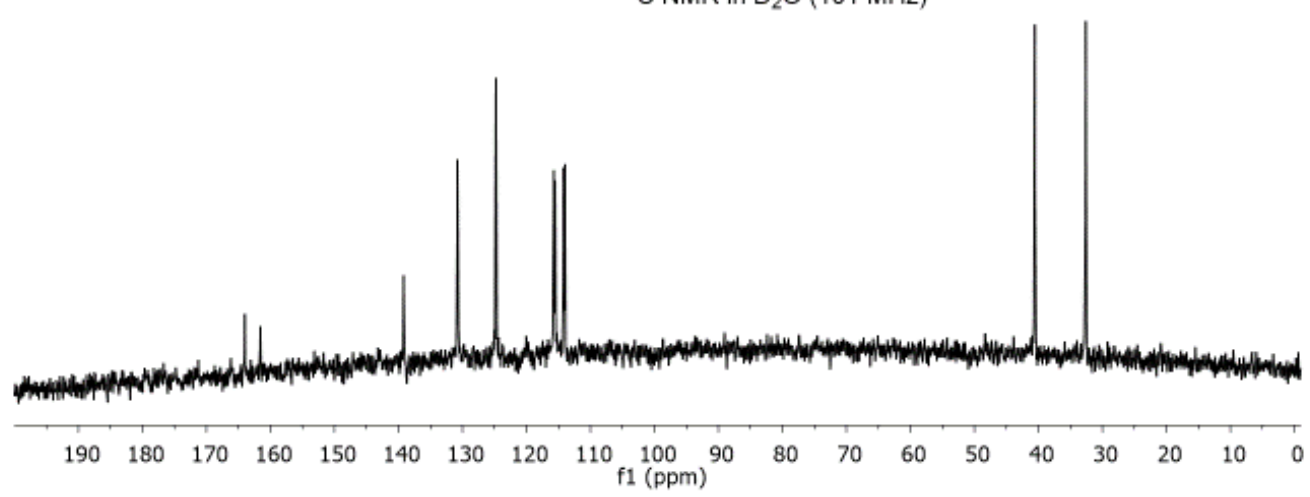




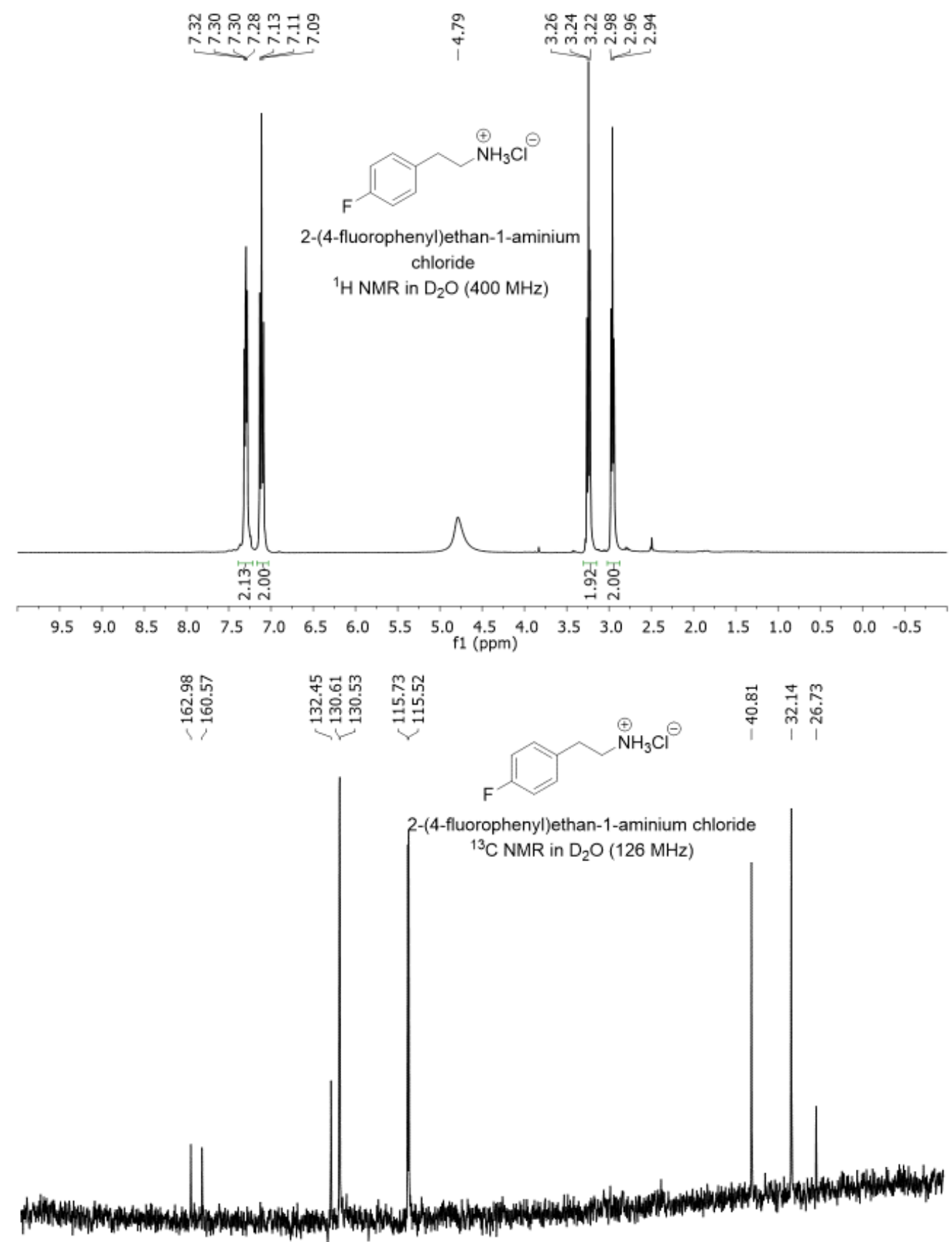

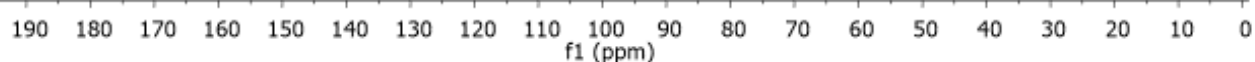



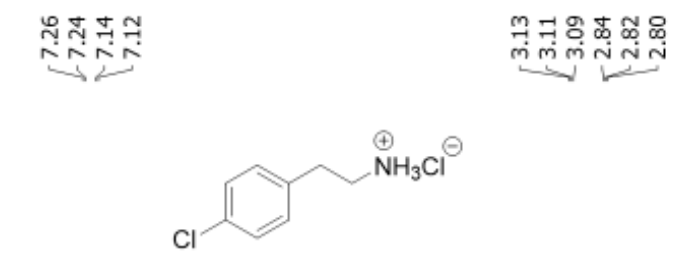

2-(4-chlorophenyl)ethan-1-aminium chloride

${ }^{1} \mathrm{H} \mathrm{NMR}$ in $\mathrm{D}_{2} \mathrm{O}(400 \mathrm{MHz})$
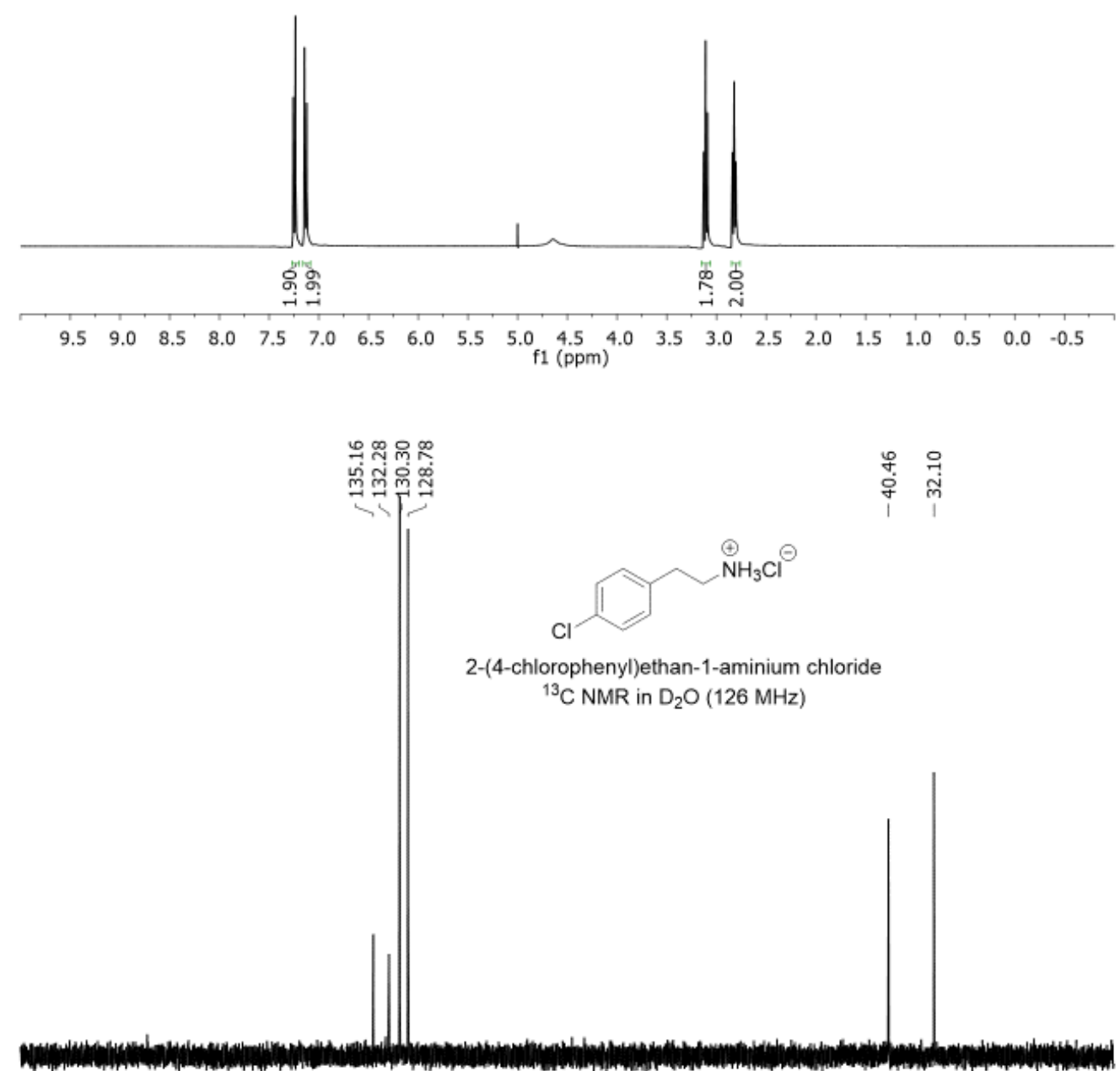

$\begin{array}{llllllllllllllllllll}200 & 190 & 180 & 170 & 160 & 150 & 140 & 130 & 120 & 110 \underset{\mathrm{f} 1(\mathrm{ppm})}{100} & 90 & 80 & 70 & 60 & 50 & 40 & 30 & 20 & 10 & 0\end{array}$ 


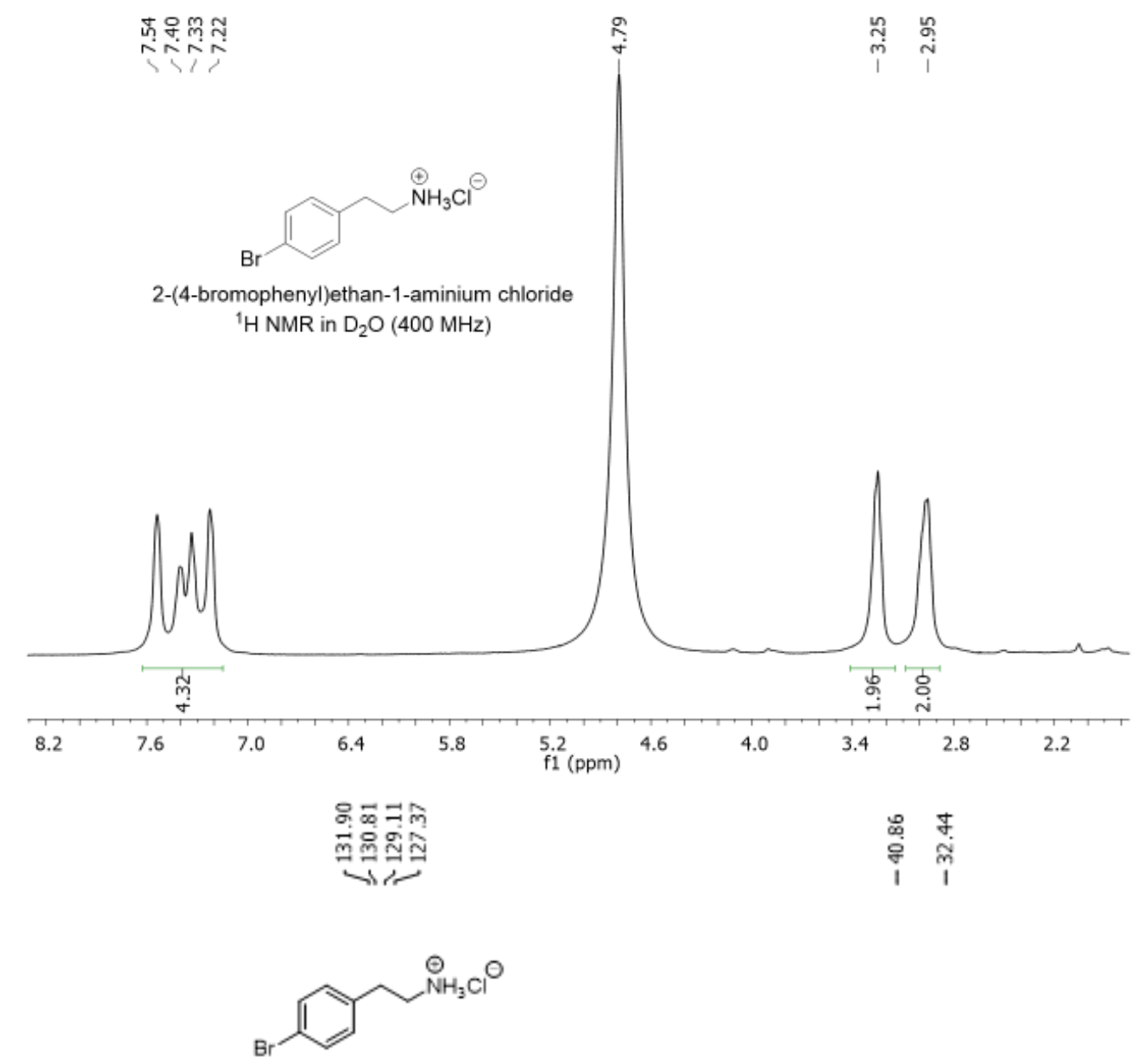

2-(4-bromophenyl)ethan-1-aminium chloride

${ }^{13} \mathrm{C}$ NMR in $\mathrm{D}_{2} \mathrm{O}(101 \mathrm{MHz})$

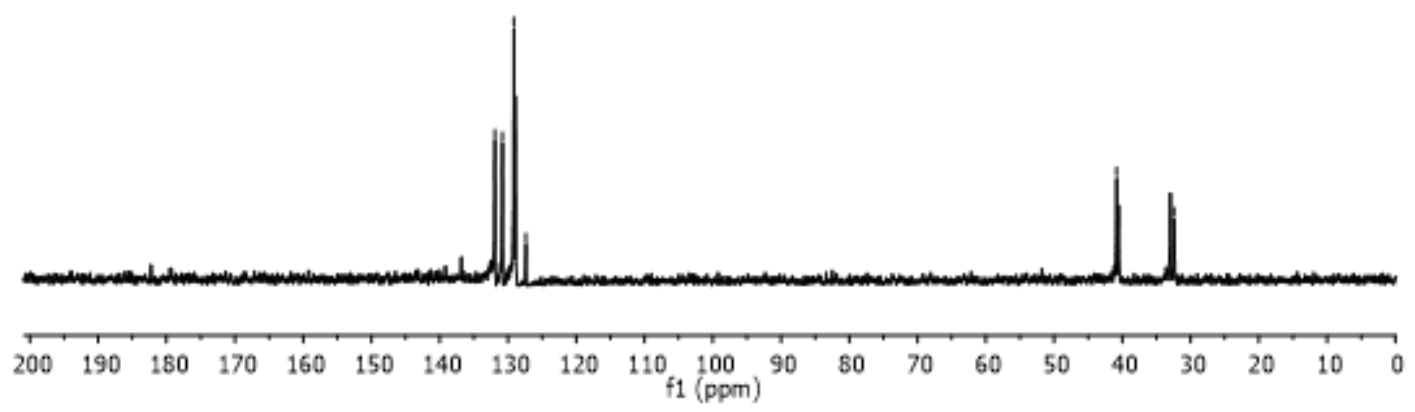




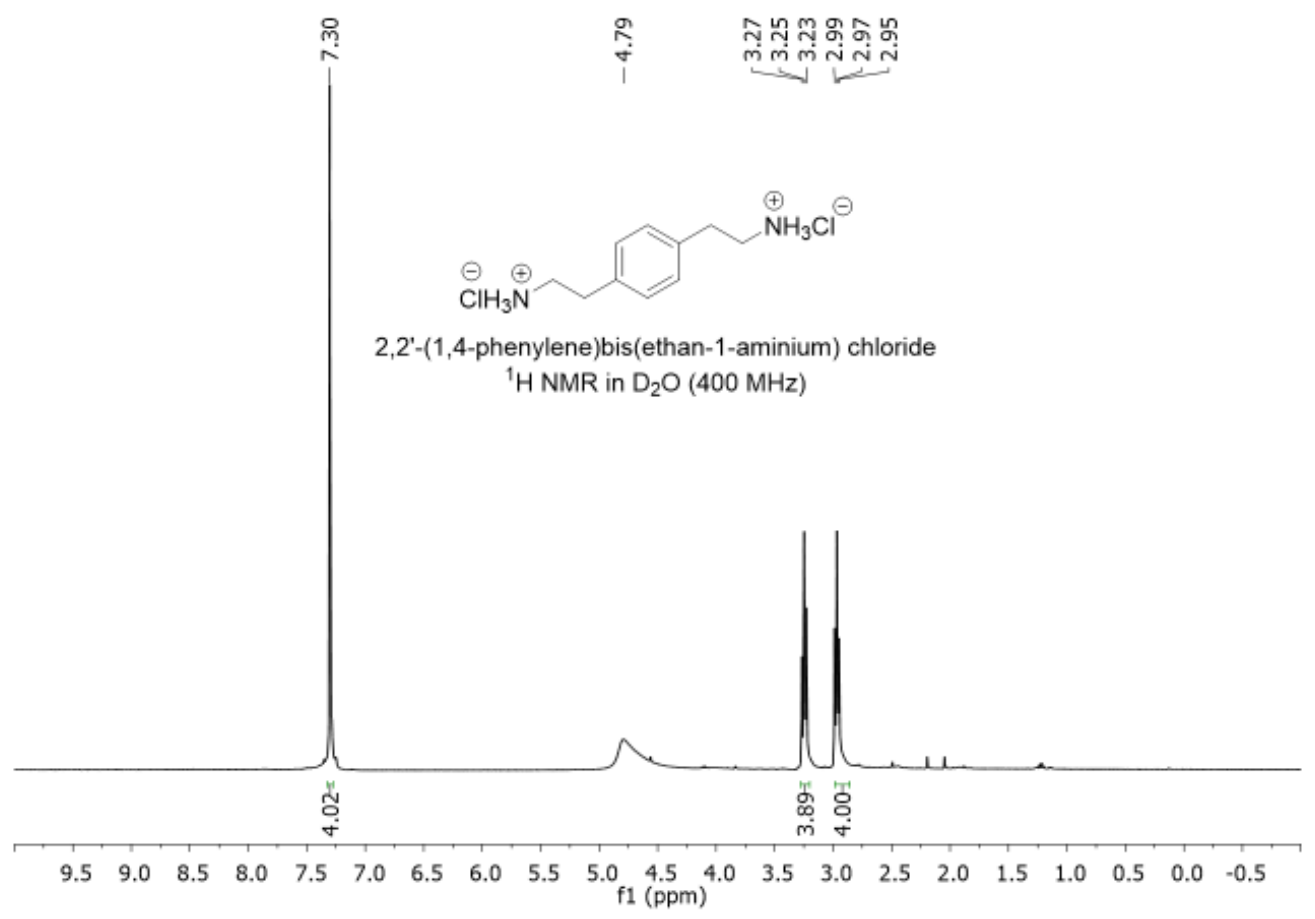

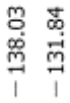

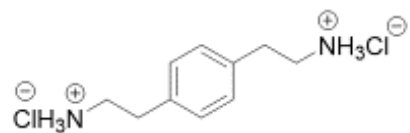

2,2'-(1,4-phenylene)bis(ethan-1-aminium) chloride ${ }^{13} \mathrm{C}$ NMR in $\mathrm{D}_{2} \mathrm{O}(101 \mathrm{MHz})$
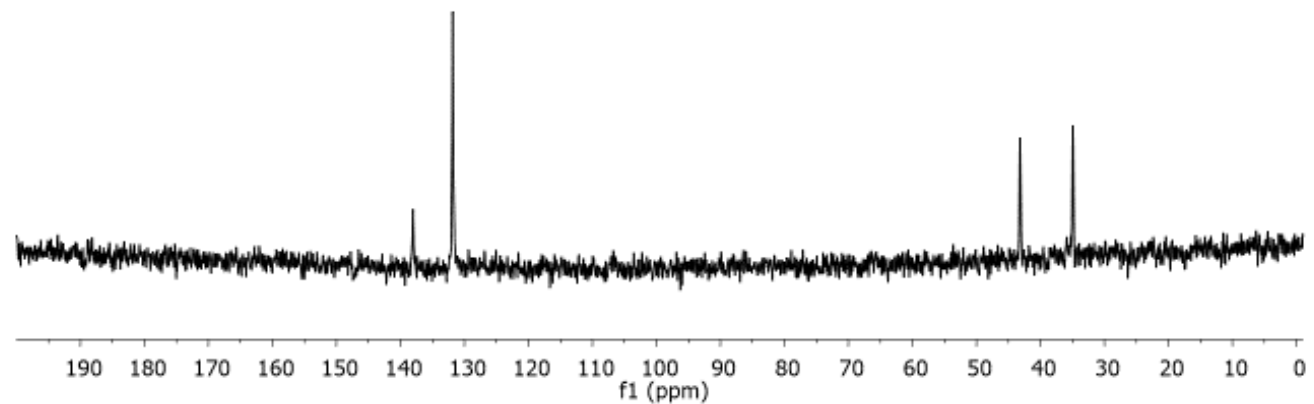

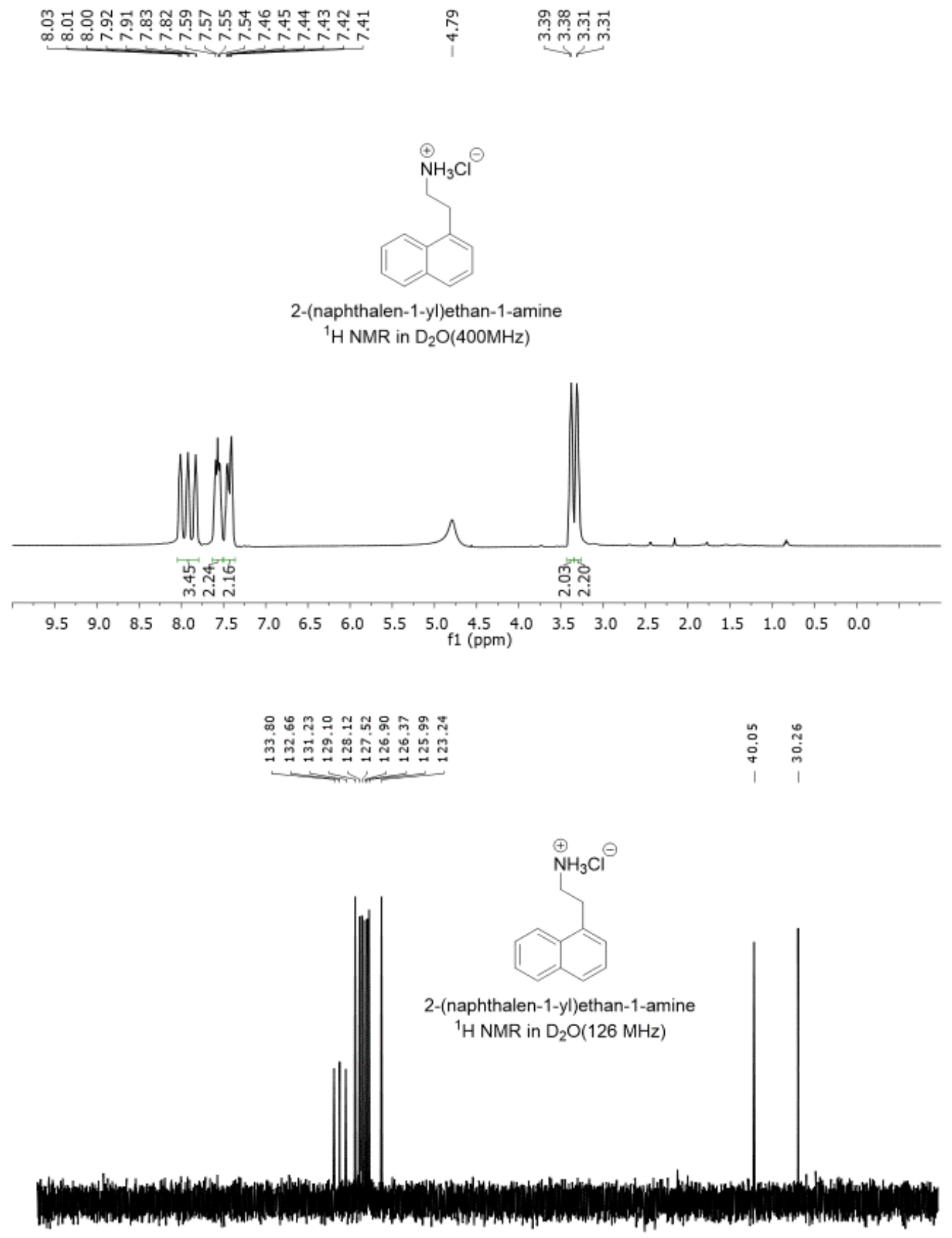

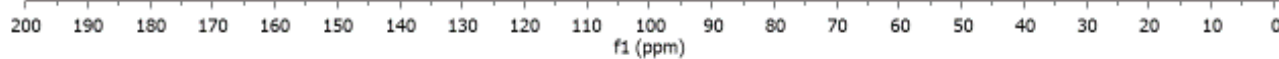




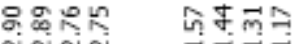

Non
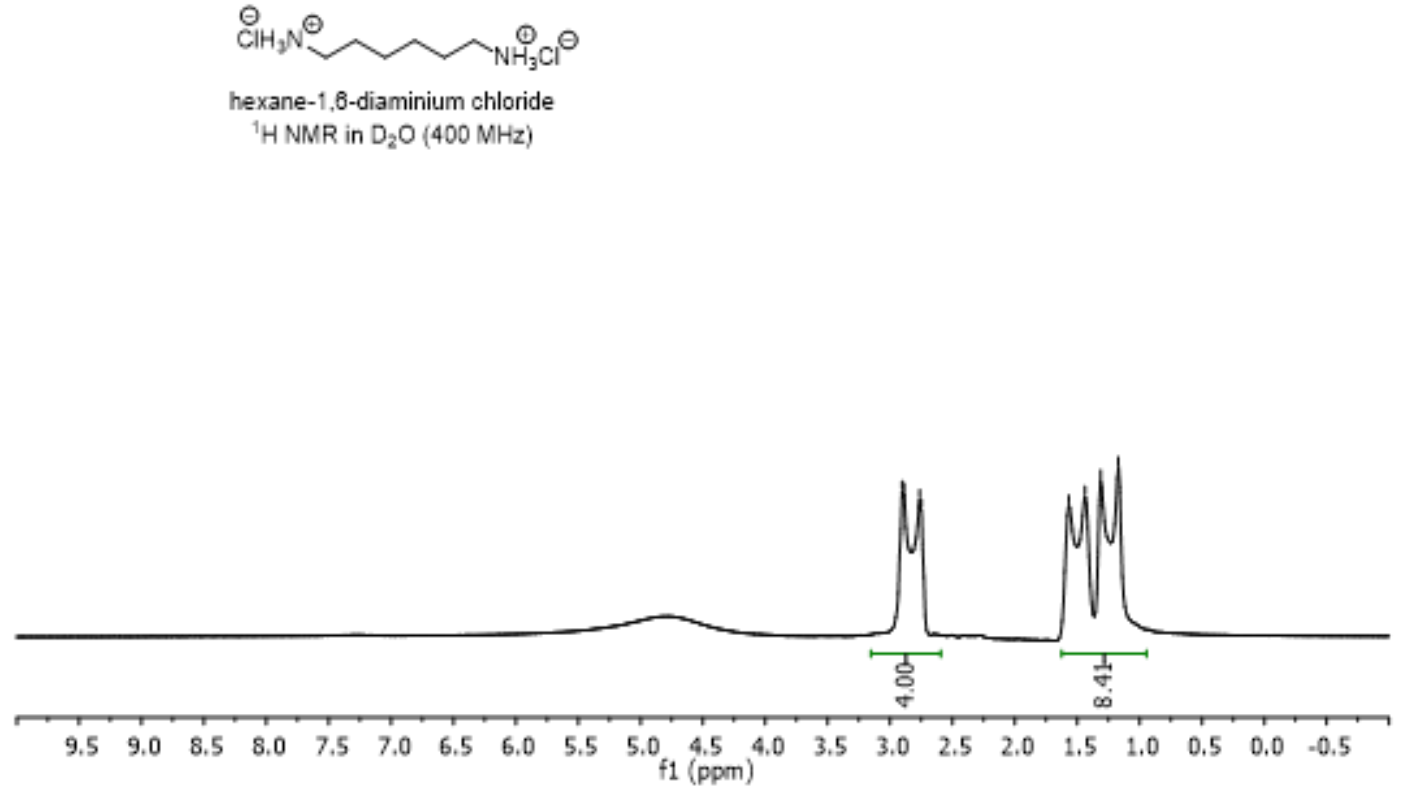

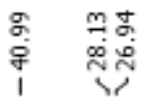

$\stackrel{\ominus}{\mathrm{ClH}_{3}} \mathrm{~N} \underbrace{\sim}_{\sim} \sim \mathrm{NH}_{3} \mathrm{Cl}^{\ominus}$

hexane-1,6-diaminium chloride

${ }^{13} \mathrm{C}$ NMR in $\mathrm{D}_{2} \mathrm{O}(101 \mathrm{MHz})$

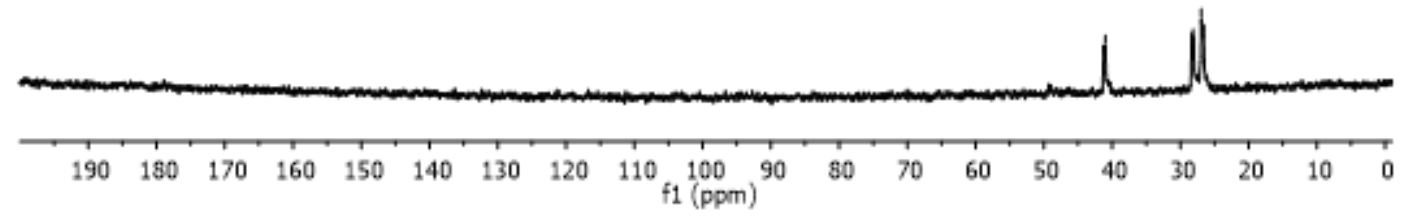


${ }^{1} \mathrm{H}$ and ${ }^{13} \mathrm{C}$ NMR Data of symmetric secondary amines:

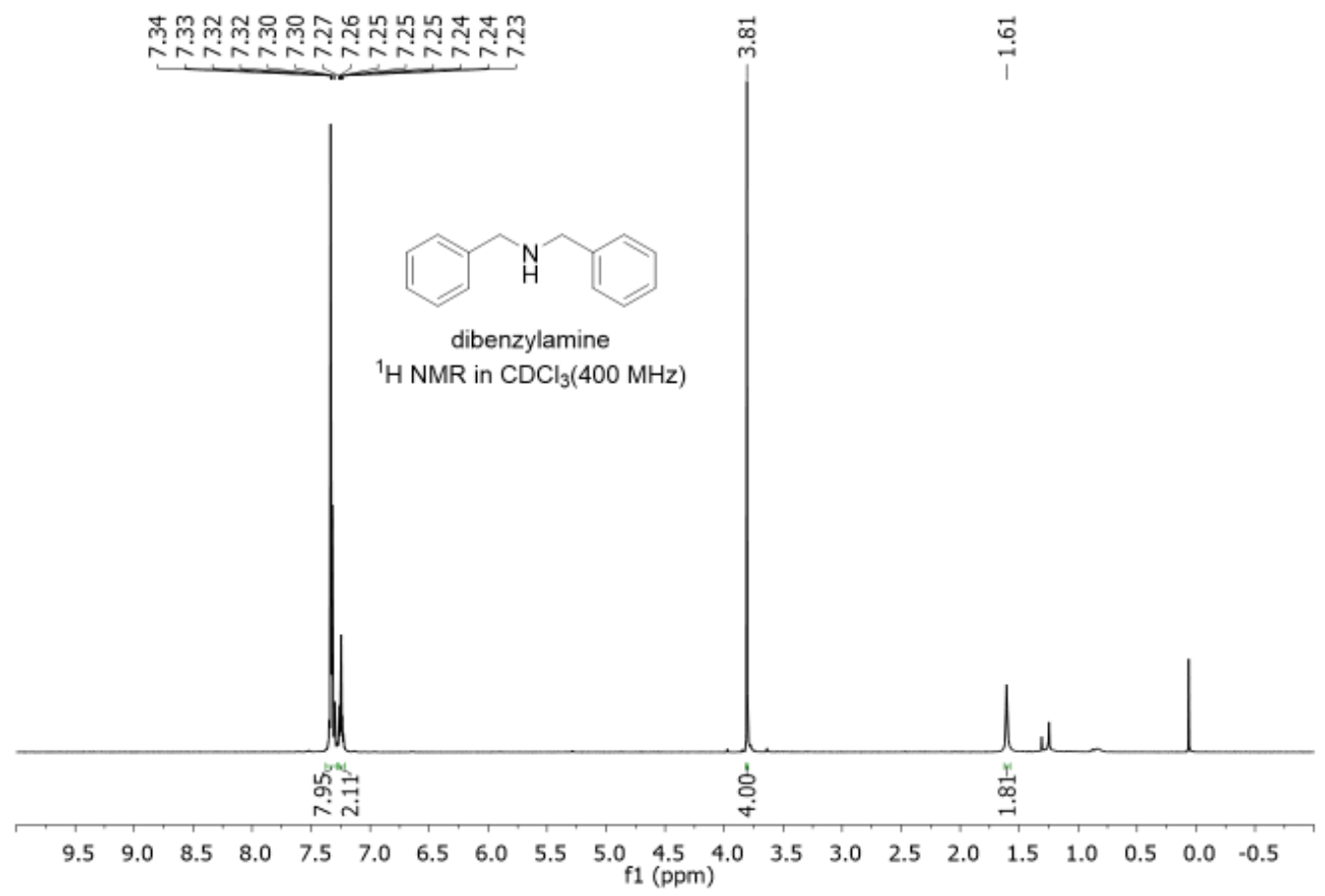

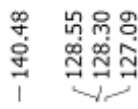

m

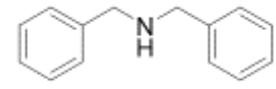

dibenzylamine

${ }^{13} \mathrm{C} \mathrm{NMR}$ in $\mathrm{CDCl}_{3}(101 \mathrm{MHz})$

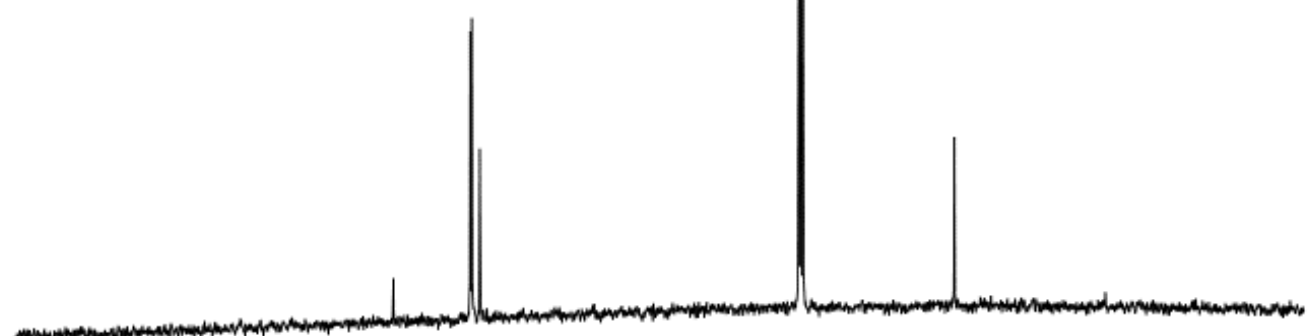

$\begin{array}{llllllllllllllllll}190 & 180 & 170 & 160 & 150 & 140 & 130 & 120 & 110 \underset{f 1(\mathrm{ppm})}{100} 90 & 80 & 70 & 60 & 50 & 40 & 30 & 20 & 10 & 0\end{array}$ 

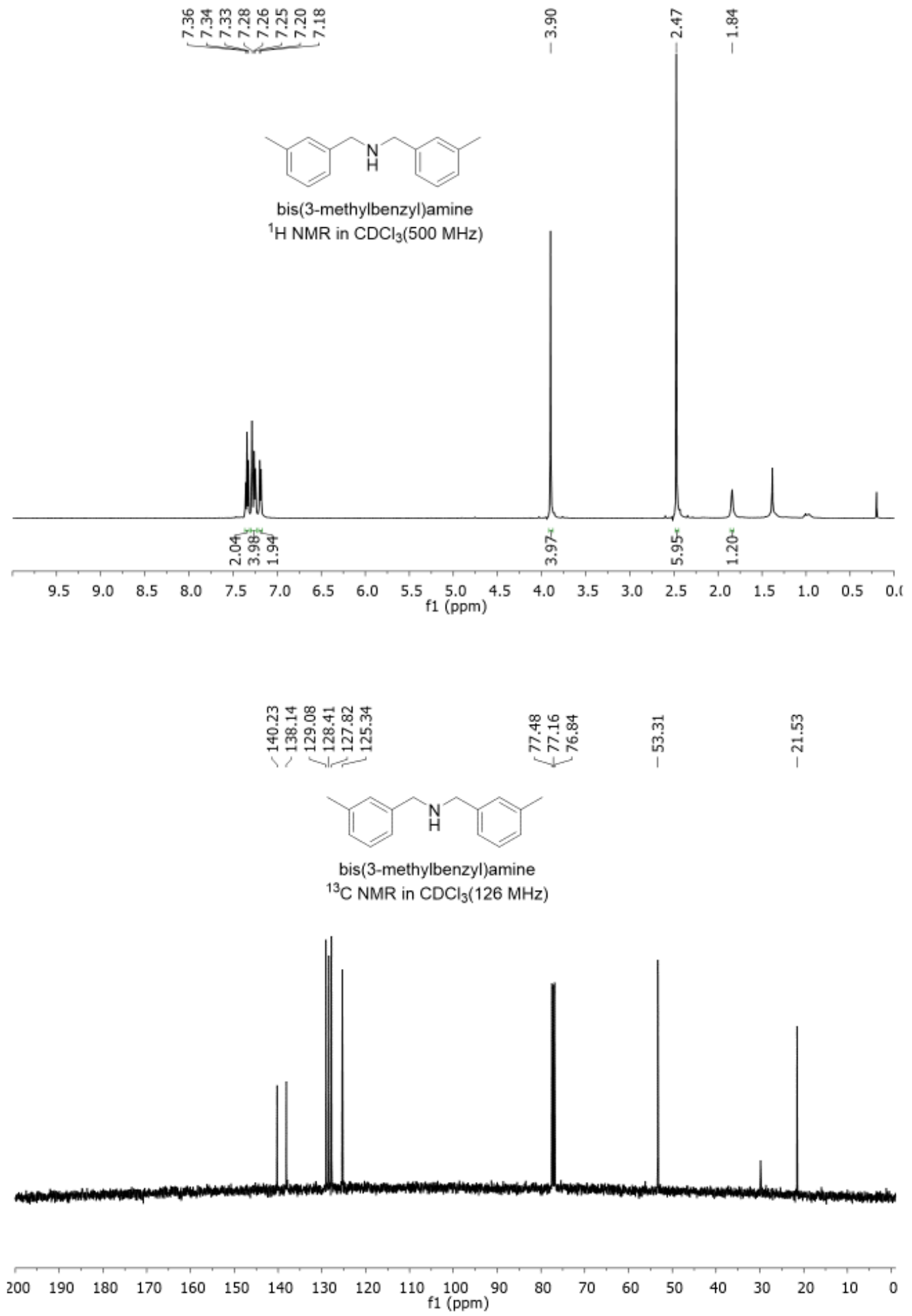

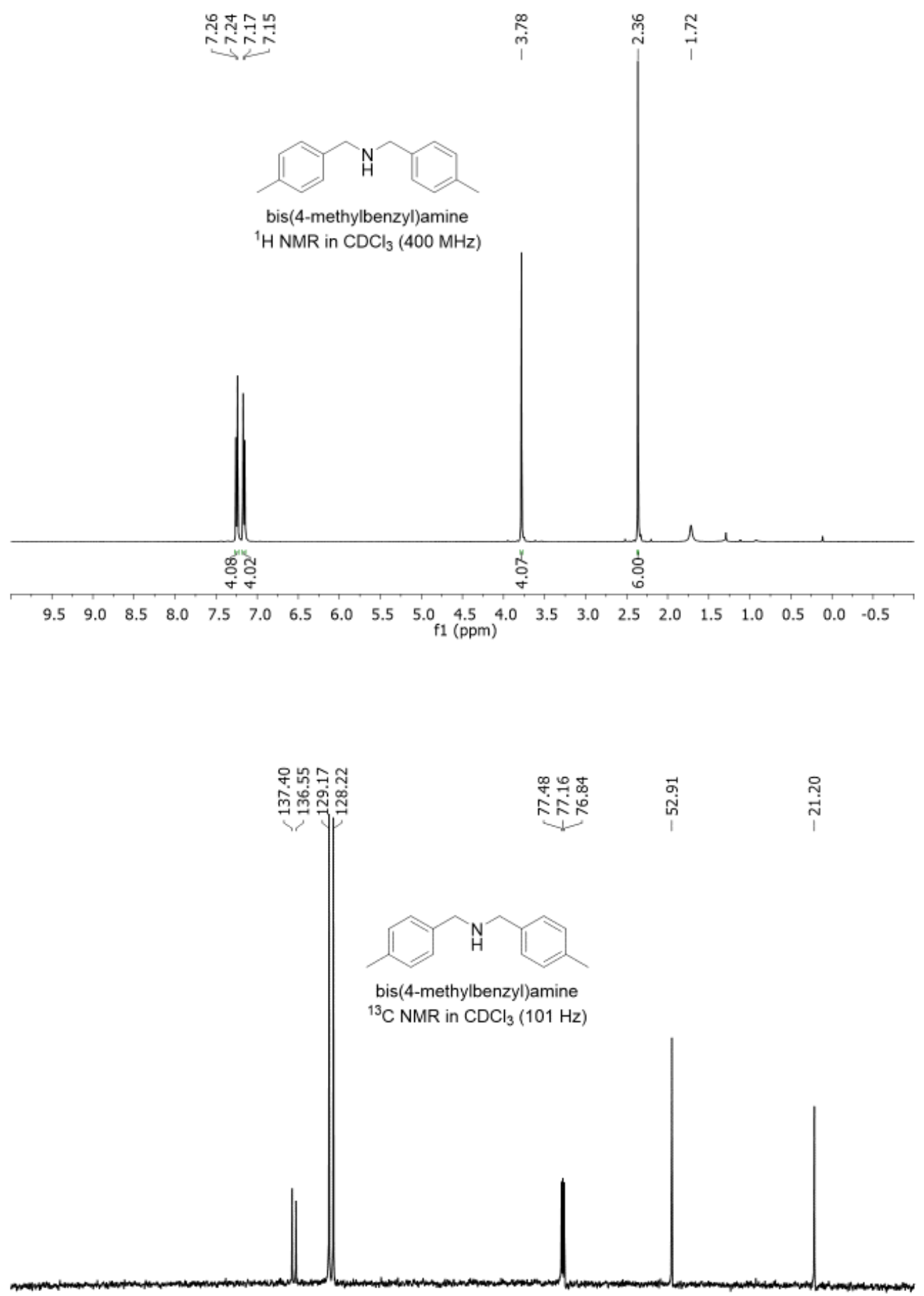

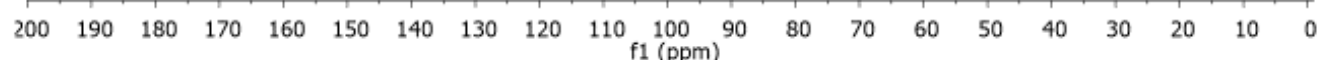




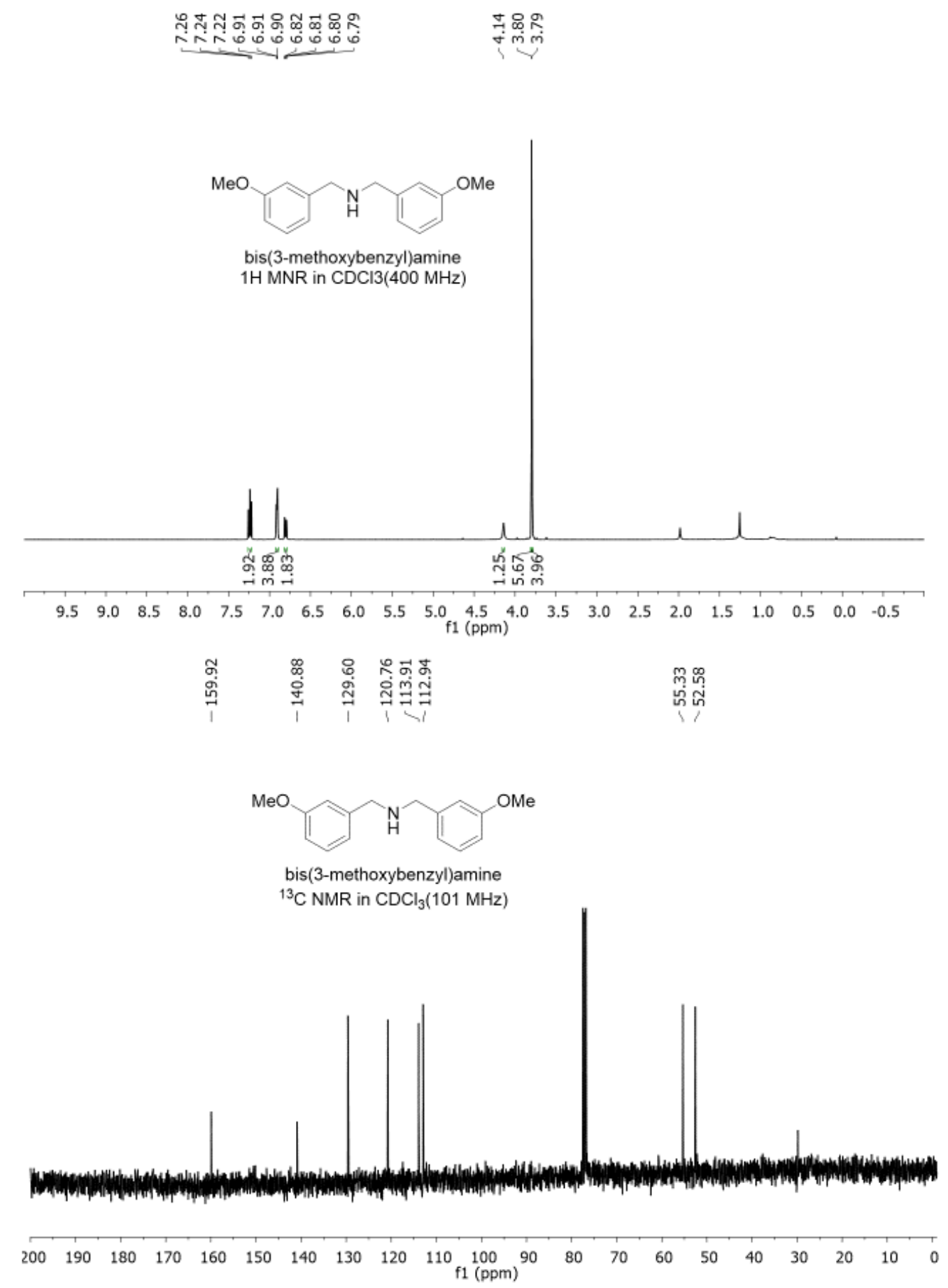




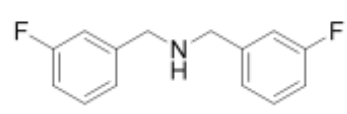

bis(3-fluorobenzyl)amine ${ }^{1} \mathrm{H} \mathrm{NMR}$ in $\mathrm{CDCl}_{3}(400 \mathrm{MHz})$

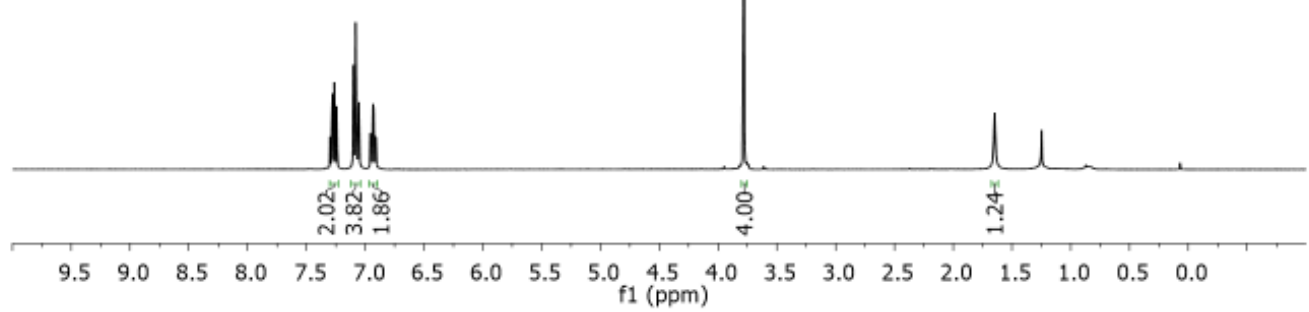

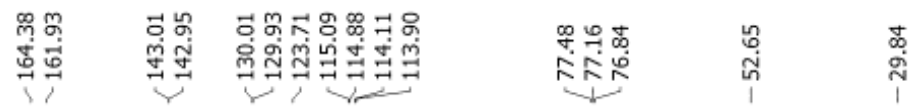

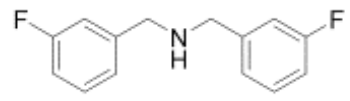

bis(3-fluorobenzyl)amine

${ }^{13} \mathrm{C} \mathrm{NMR}$ in $\mathrm{CDCl}_{3}(101 \mathrm{MHz})$

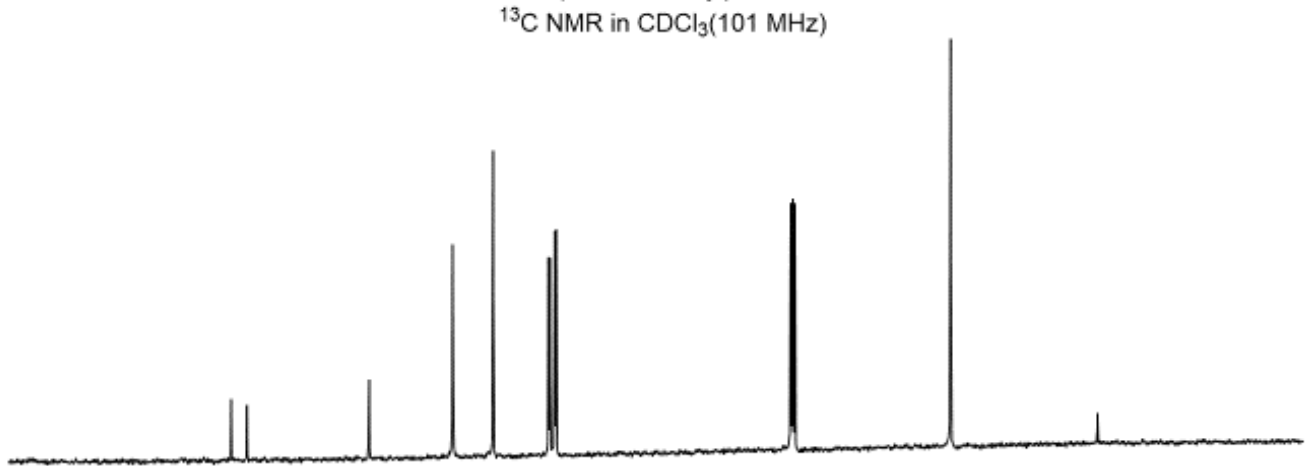

$\begin{array}{llllllllllllllllllll}190 & 180 & 170 & 160 & 150 & 140 & 130 & 120 & 110 & 100 & 90 & 80 & 70 & 60 & 50 & 40 & 30 & 20 & 10 & 0\end{array}$ 

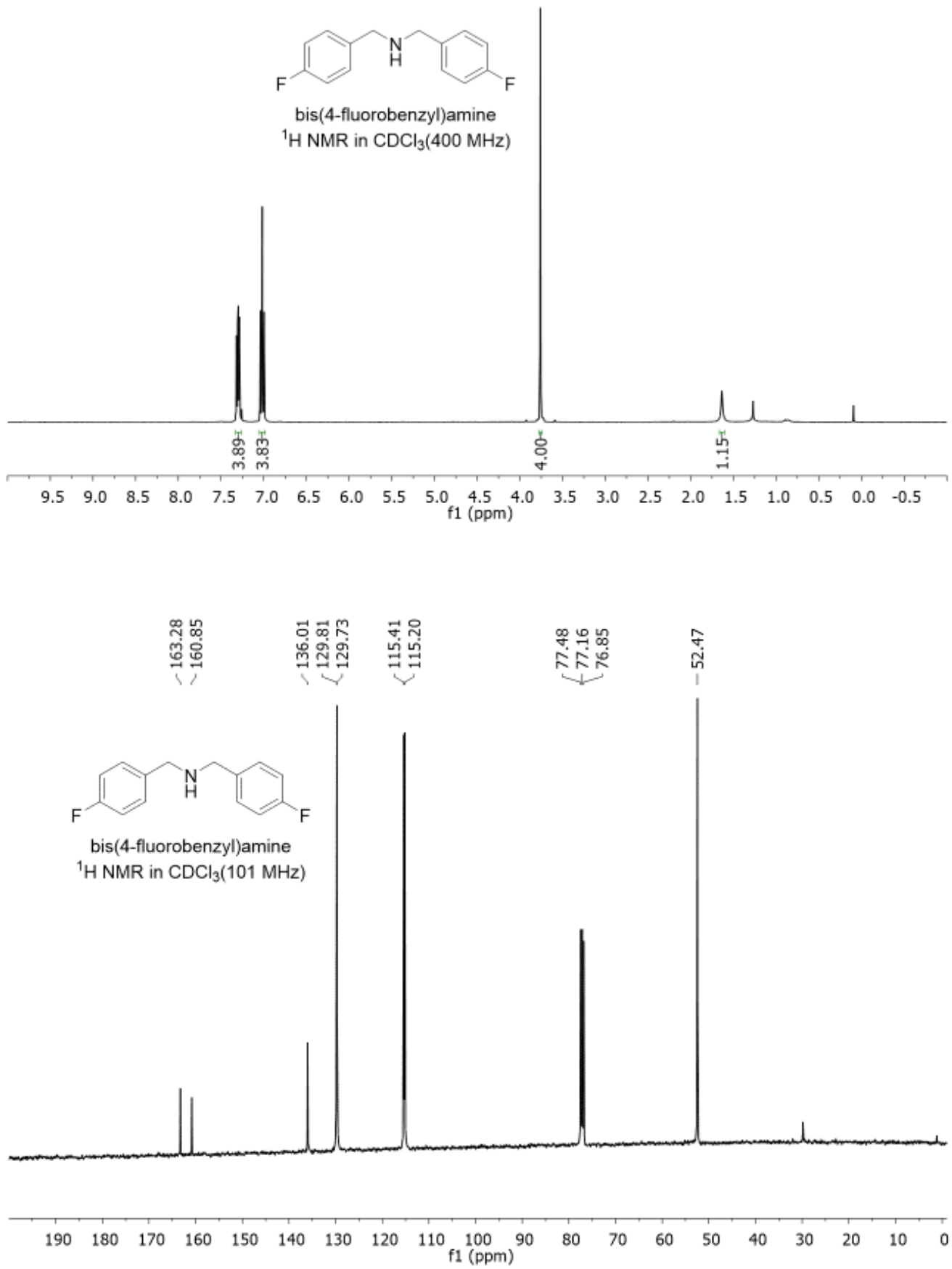


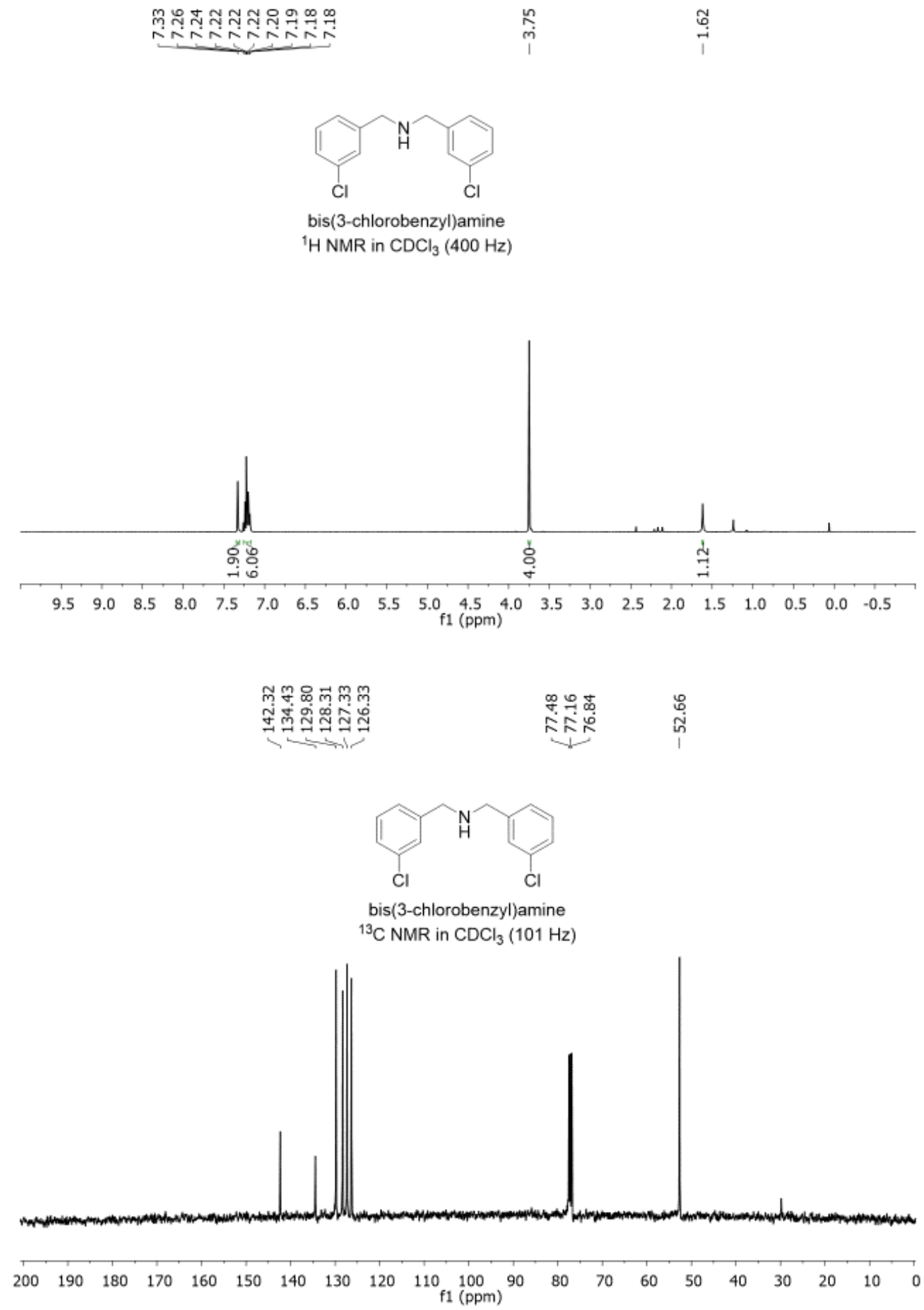



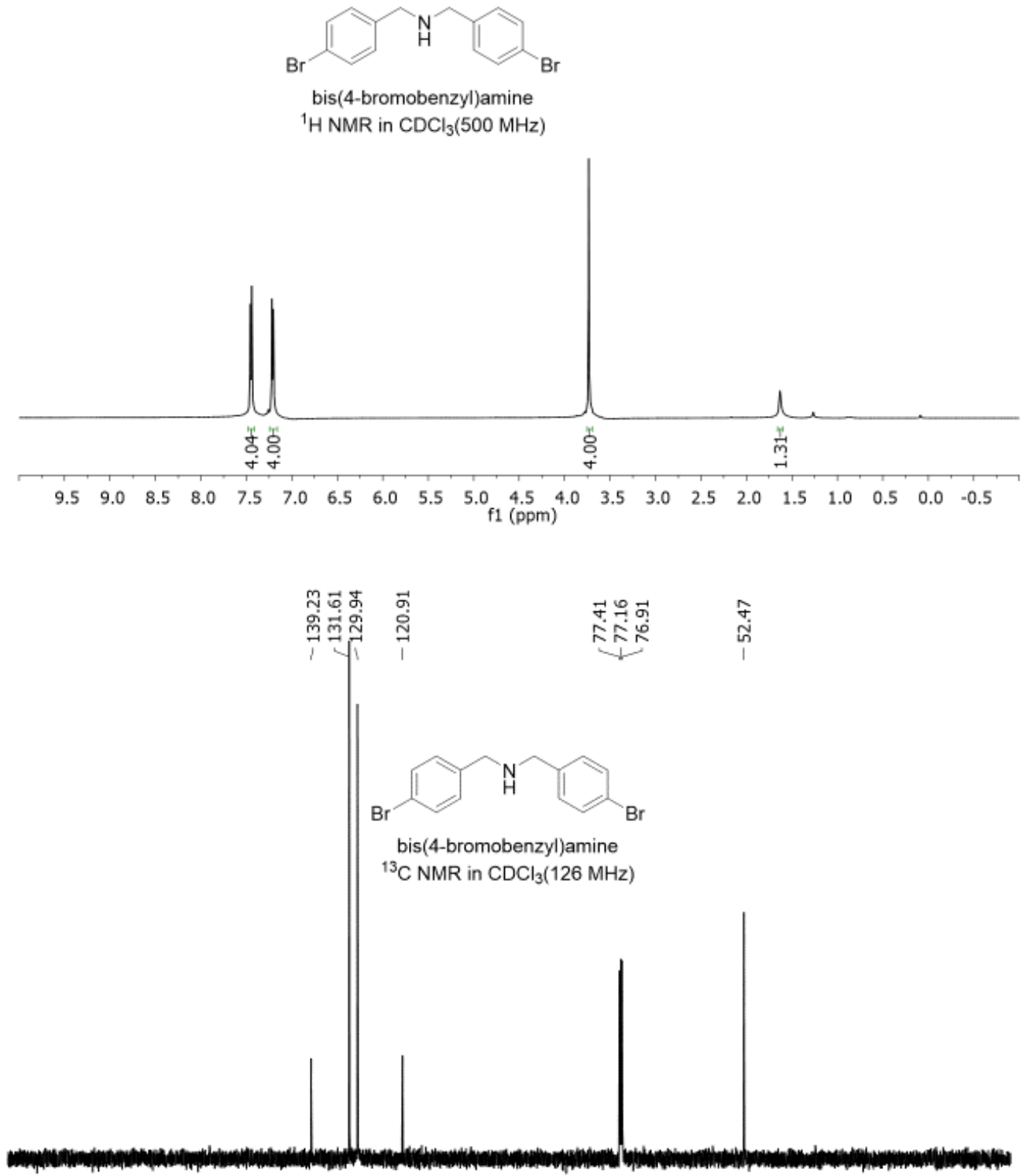

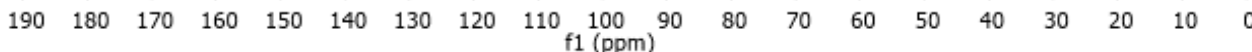




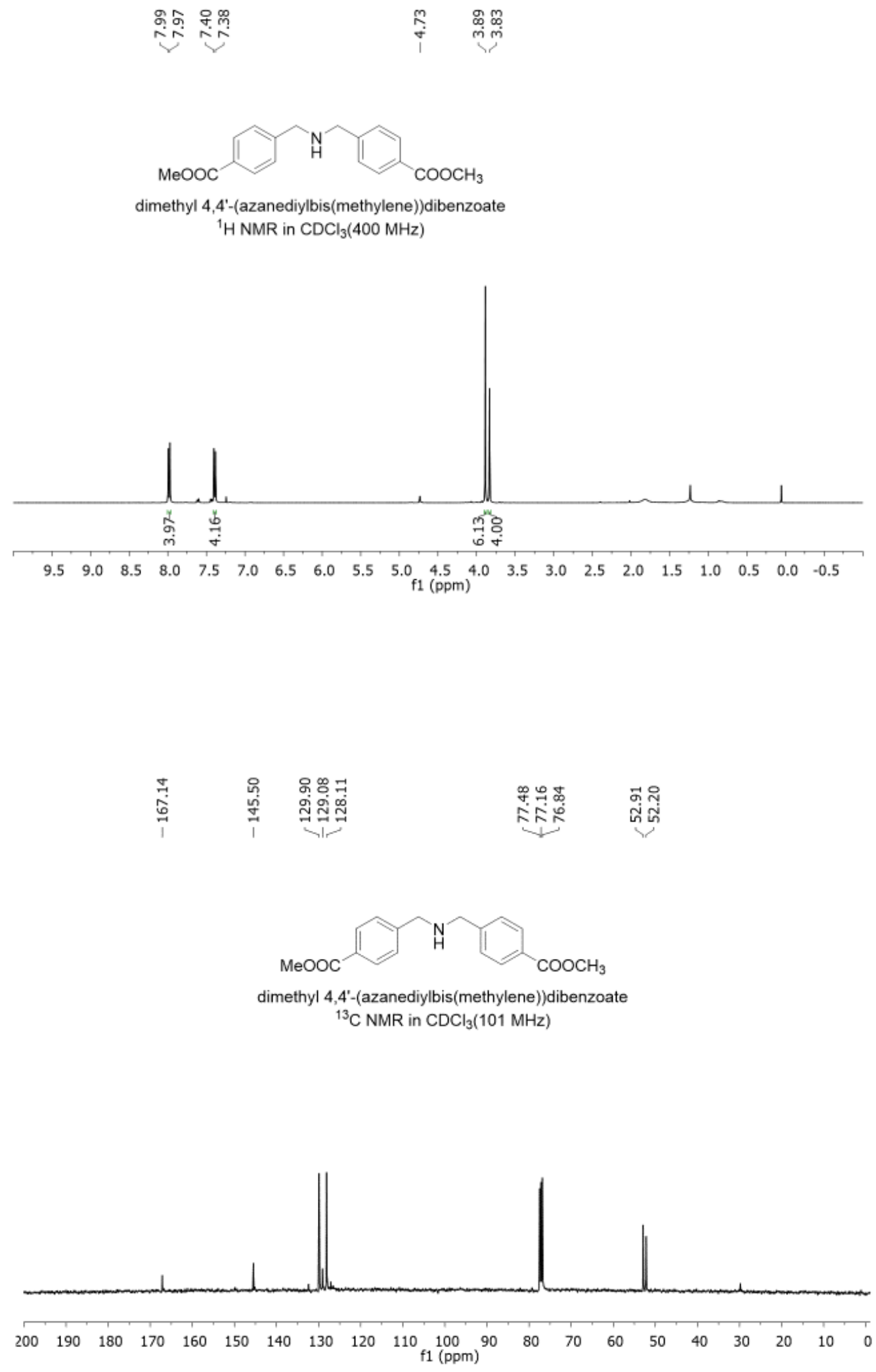

\section{${ }^{1} \mathrm{H}$ and ${ }^{13} \mathrm{C}$ NMR Data of asymmetric secondary amines:}



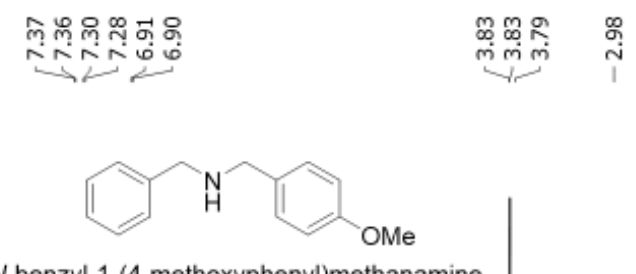

$\mathrm{N}$-benzyl-1-(4-methoxyphenyl)methanamine

${ }^{1} \mathrm{H}$ NMR data in $\mathrm{CDCl}_{3}(500 \mathrm{MHz})$
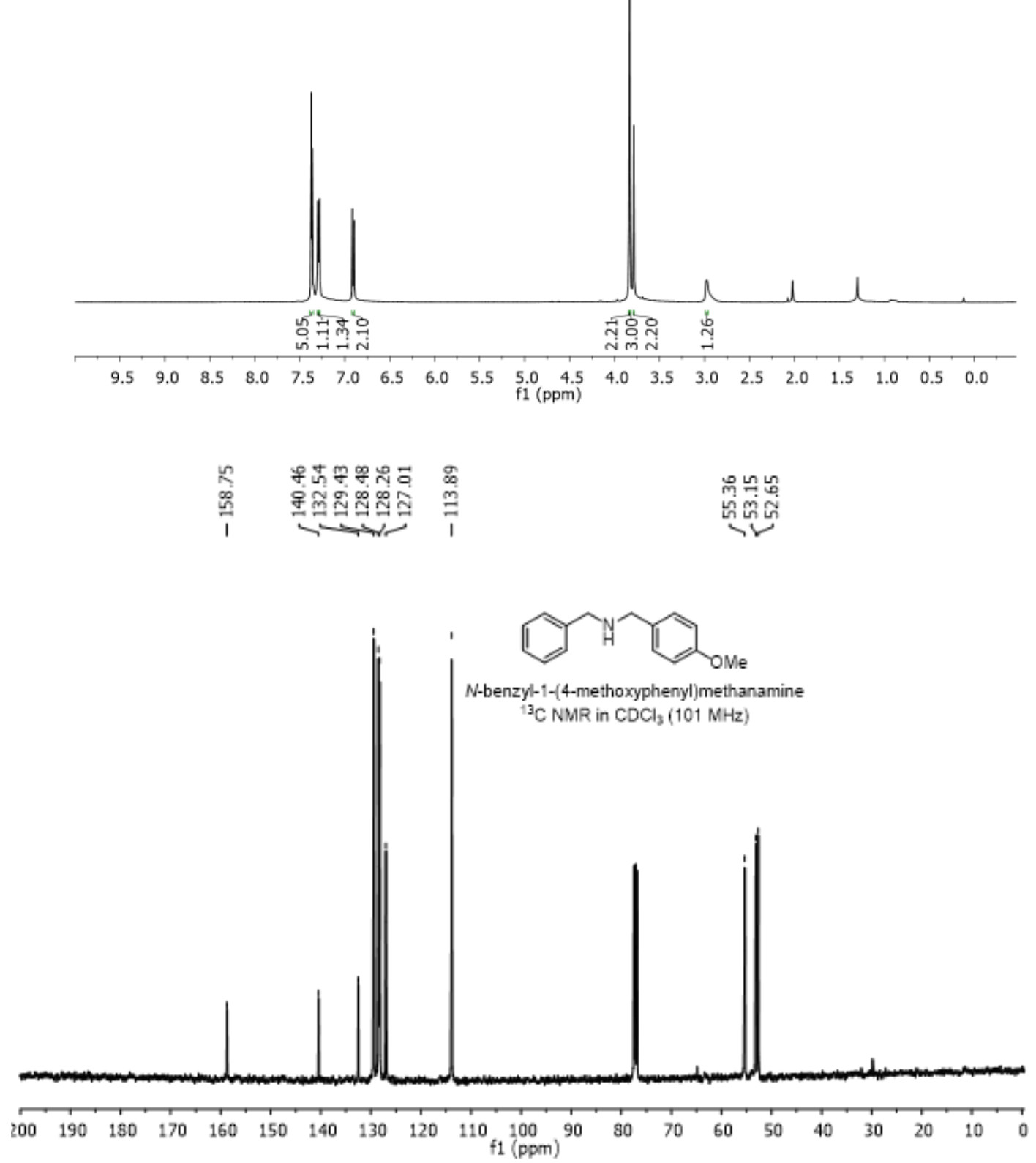


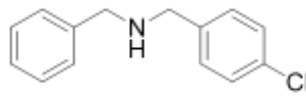

$\mathrm{N}$-benzyl-1-(4-chlorophenyl)methanamine ${ }^{1} \mathrm{H} \mathrm{NMR}$ in $\mathrm{CDCl}_{3}(500 \mathrm{MHz})$
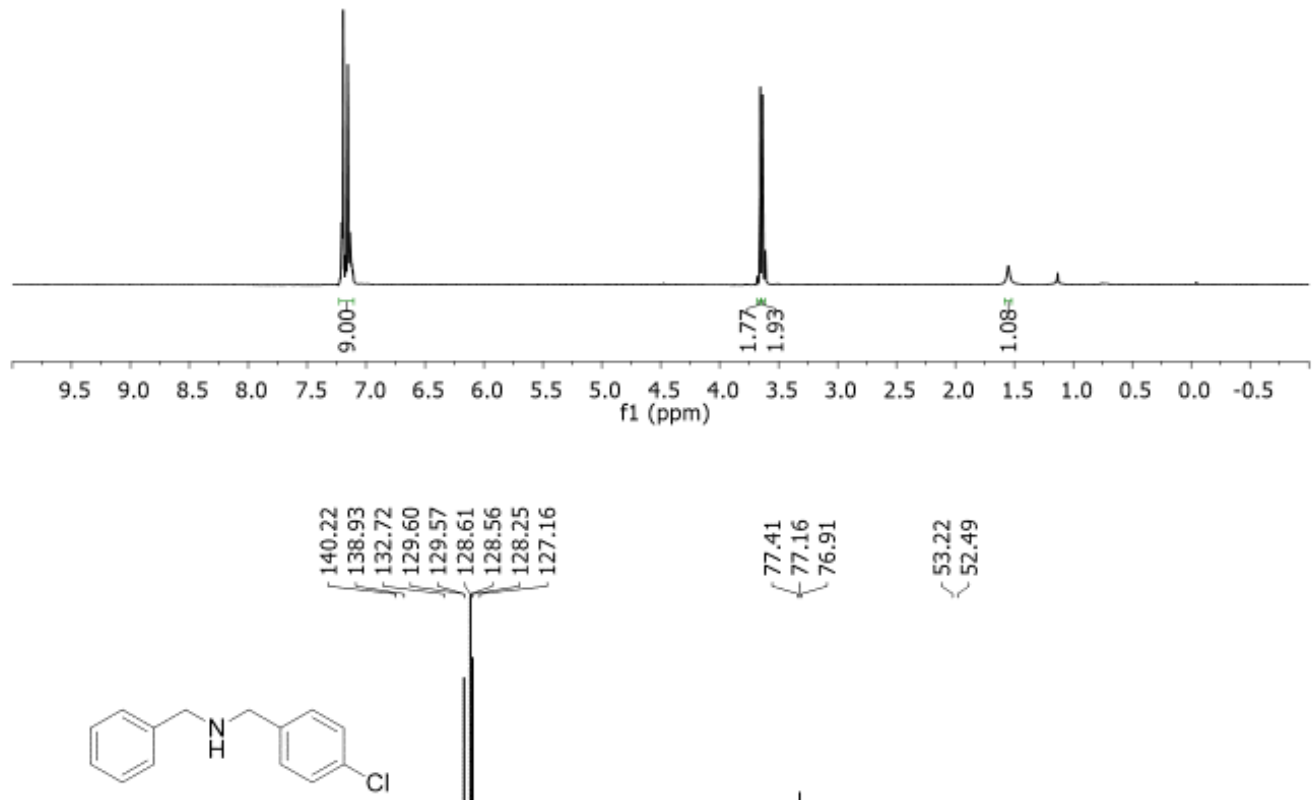

$\mathrm{N}$-benzyl-1-(4-chlorophenyl)methanamin ${ }^{13} \mathrm{C}$ NMR in $\mathrm{CDCl}_{3}(126 \mathrm{MHz})$

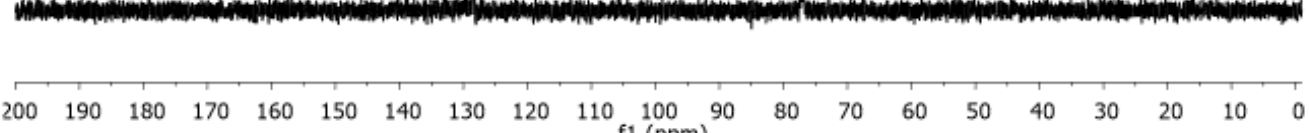



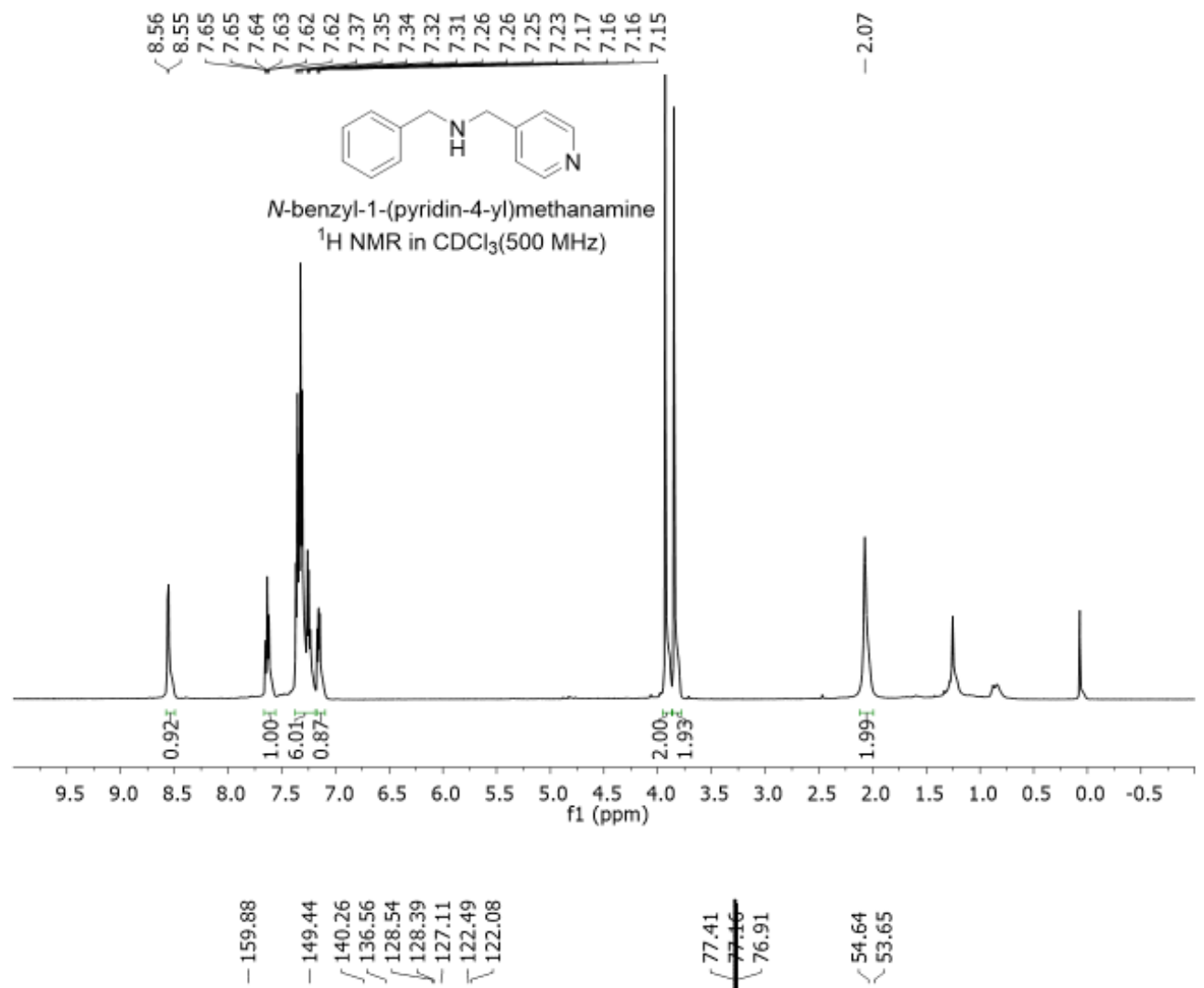

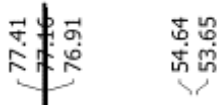

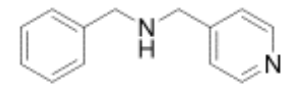

$\mathrm{N}$-benzyl-1-(pyridin-4-yl)methanamine

${ }^{13} \mathrm{C}$ NMR in $\mathrm{CDCl}_{3}(126 \mathrm{MHz})$

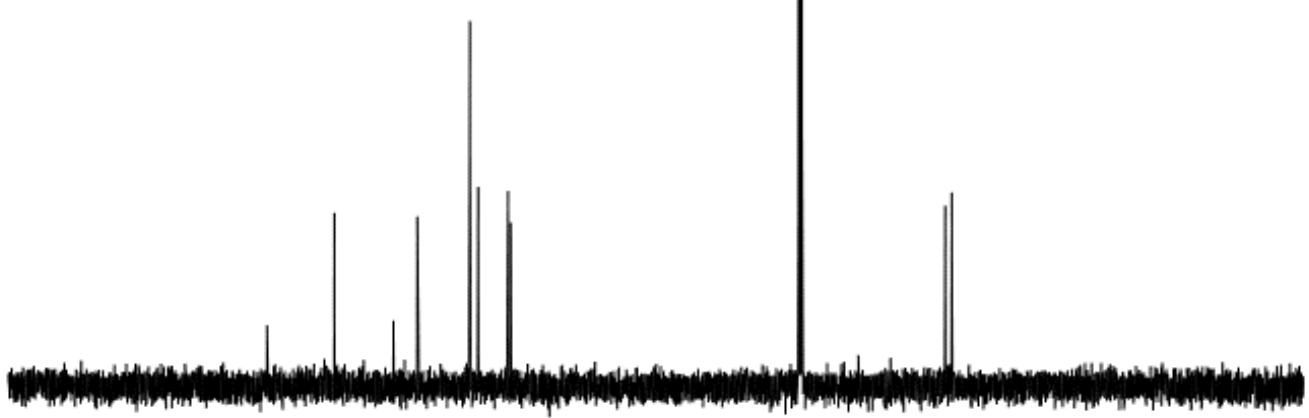

200

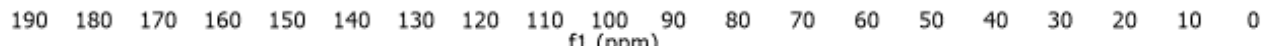



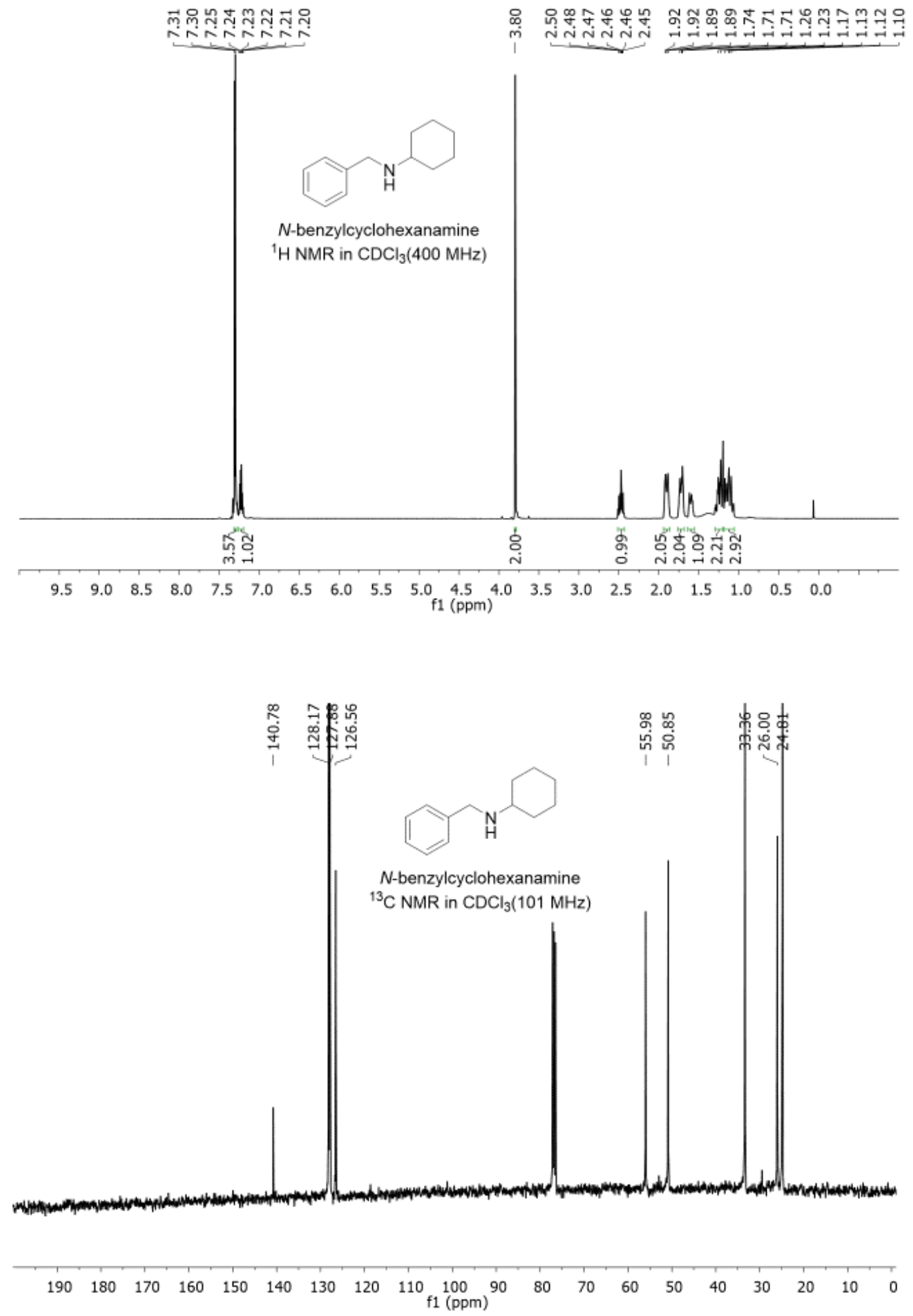

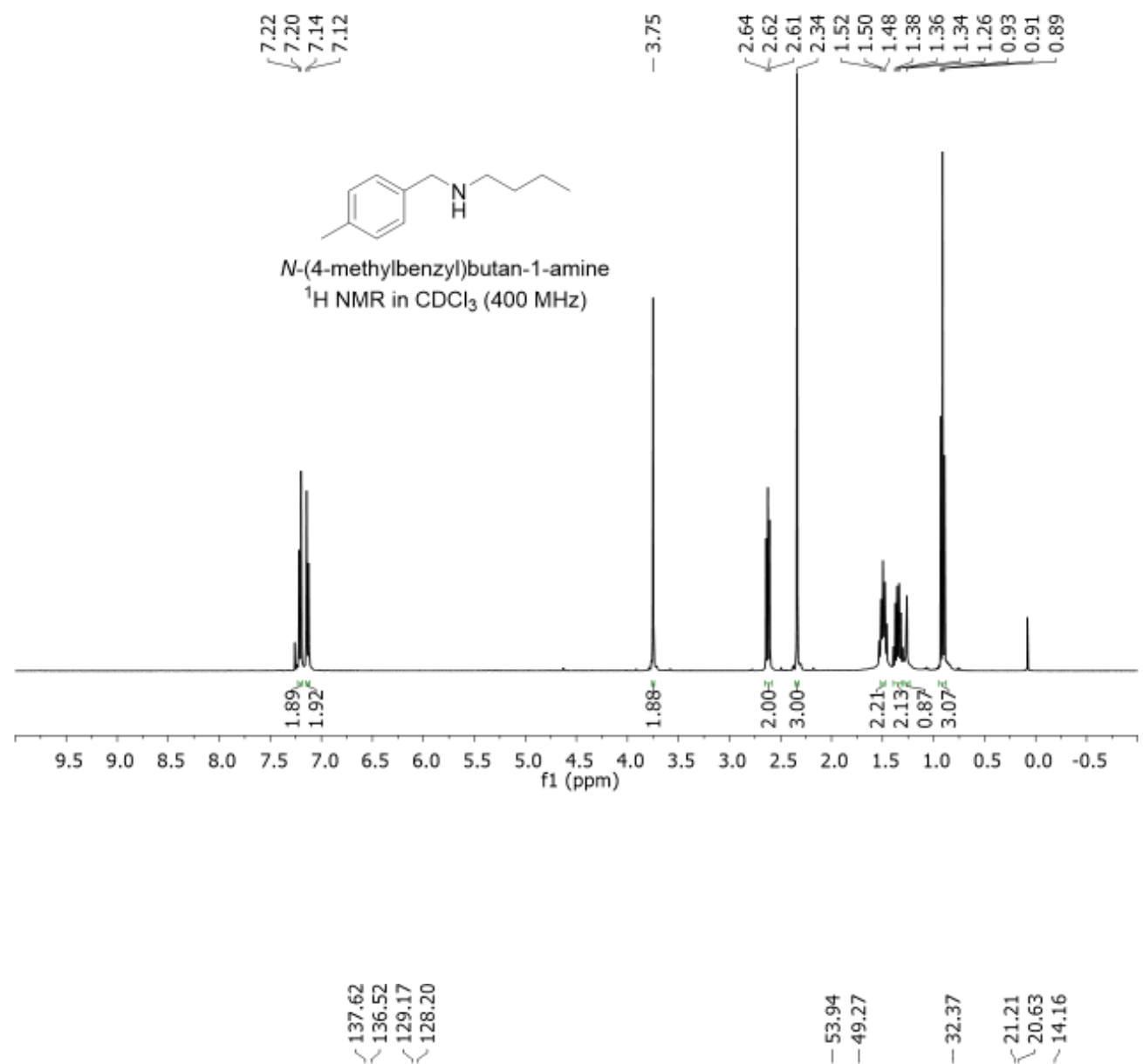

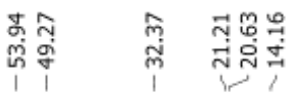

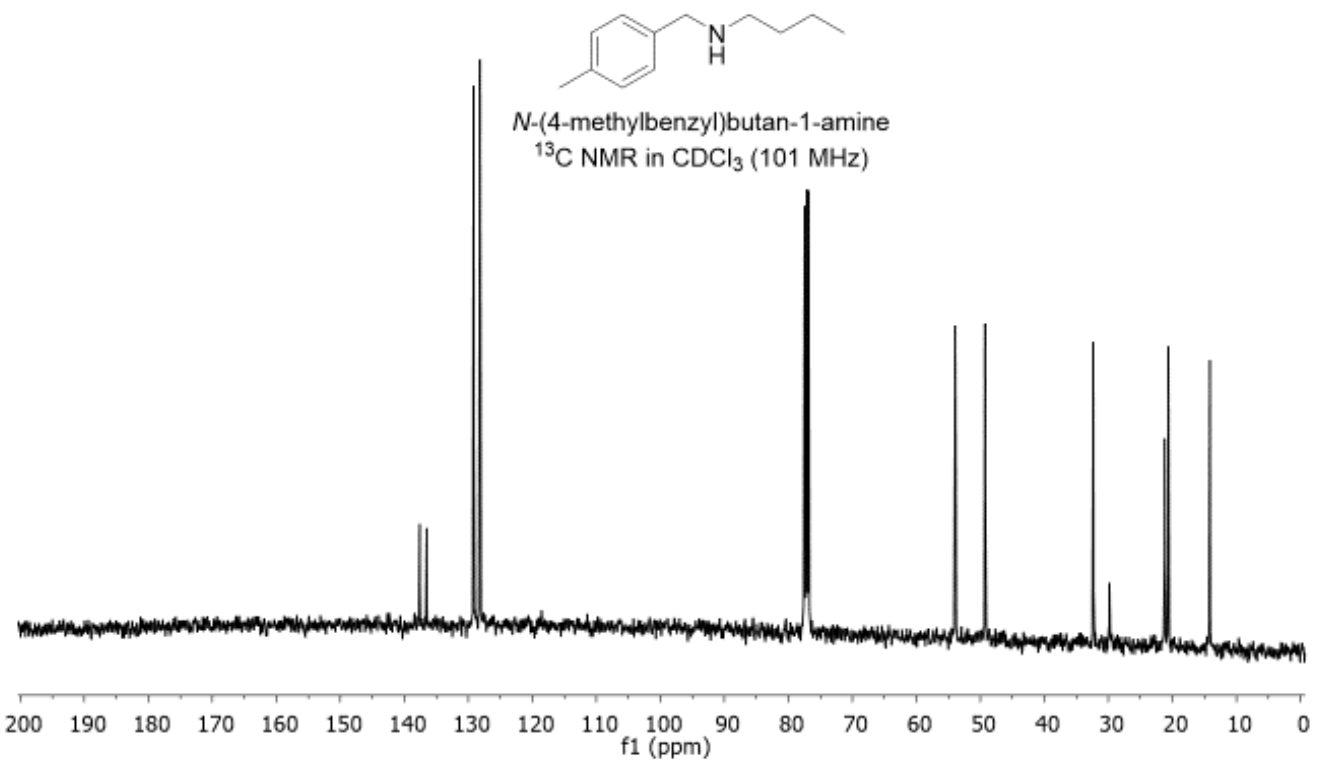




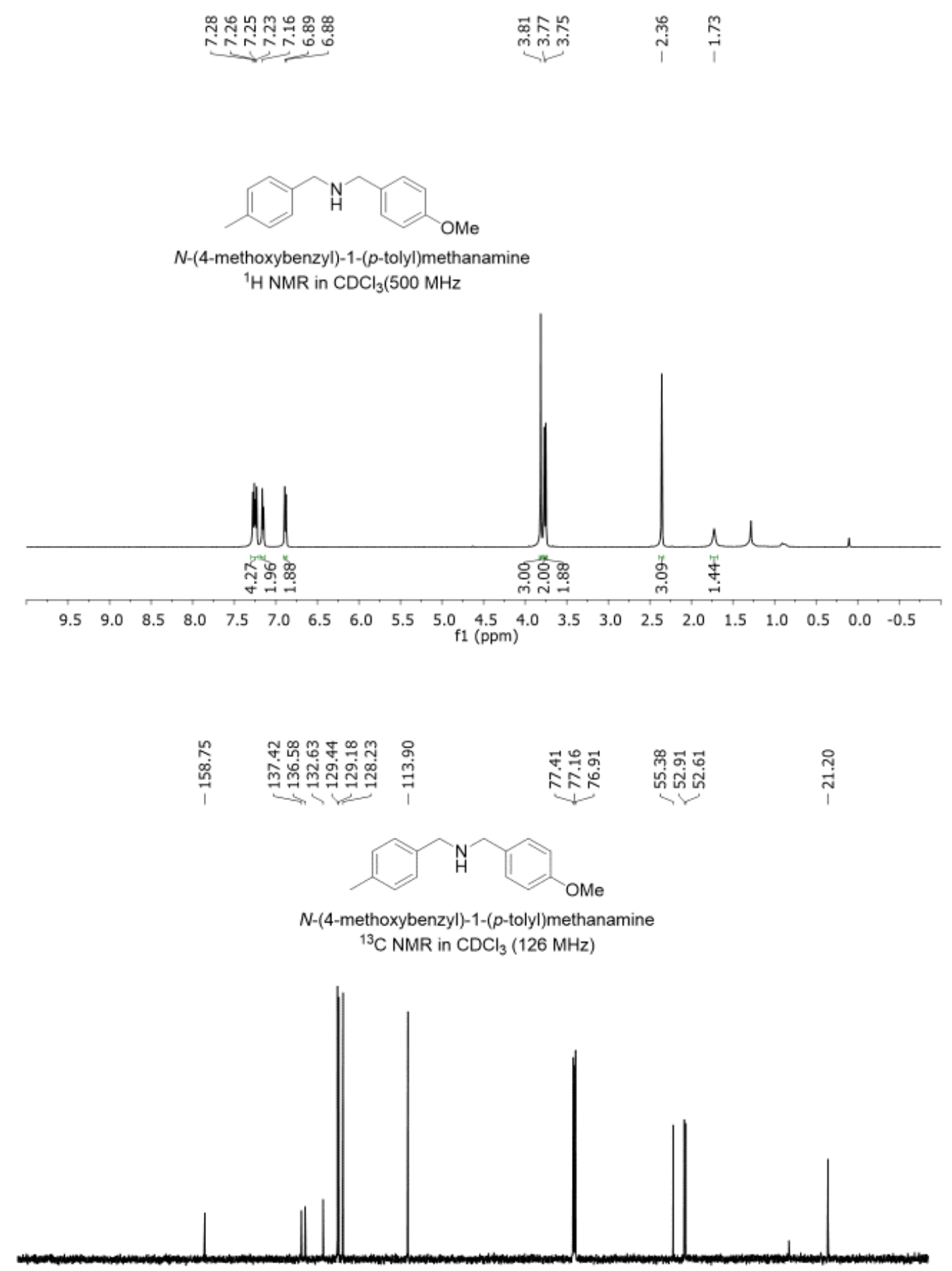

$\begin{array}{llllllllllllllllllll}200 & 190 & 180 & 170 & 160 & 150 & 140 & 130 & 120 & 110 & \underset{\mathrm{f} 1}{(100}(\mathrm{ppm}) \\ & 90 & 80 & 70 & 60 & 50 & 40 & 30 & 20 & 10 & 0\end{array}$ 


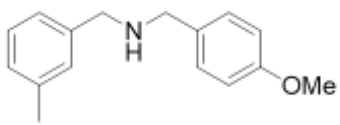

$N$-(4-methoxybenzyl)-1-( $m$-tolyl)methanamine ${ }^{1} \mathrm{H} \mathrm{NMR}$ in $\mathrm{CDCl}_{3}(500 \mathrm{MHz})$
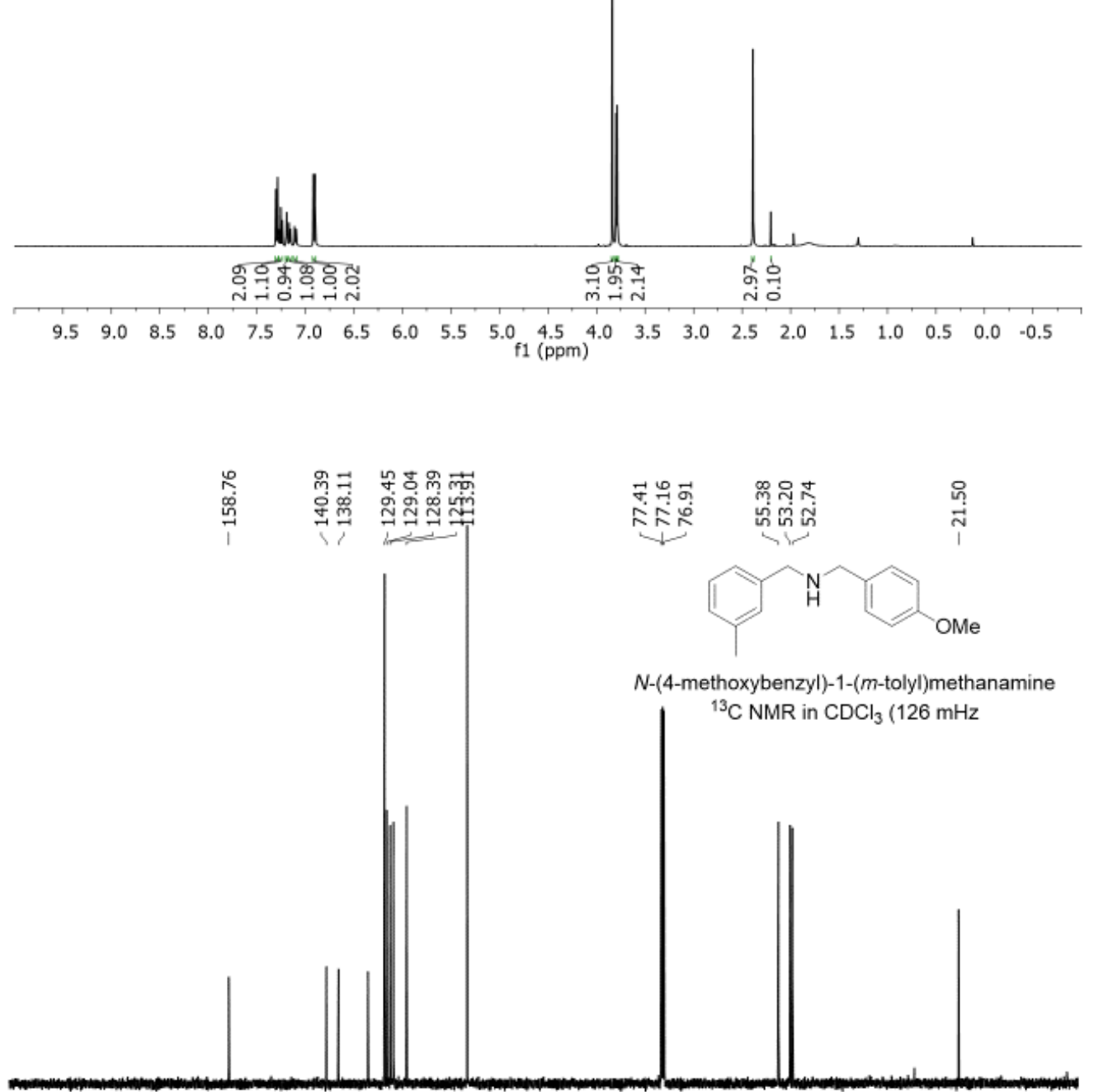

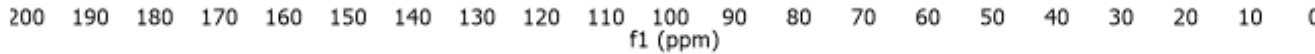



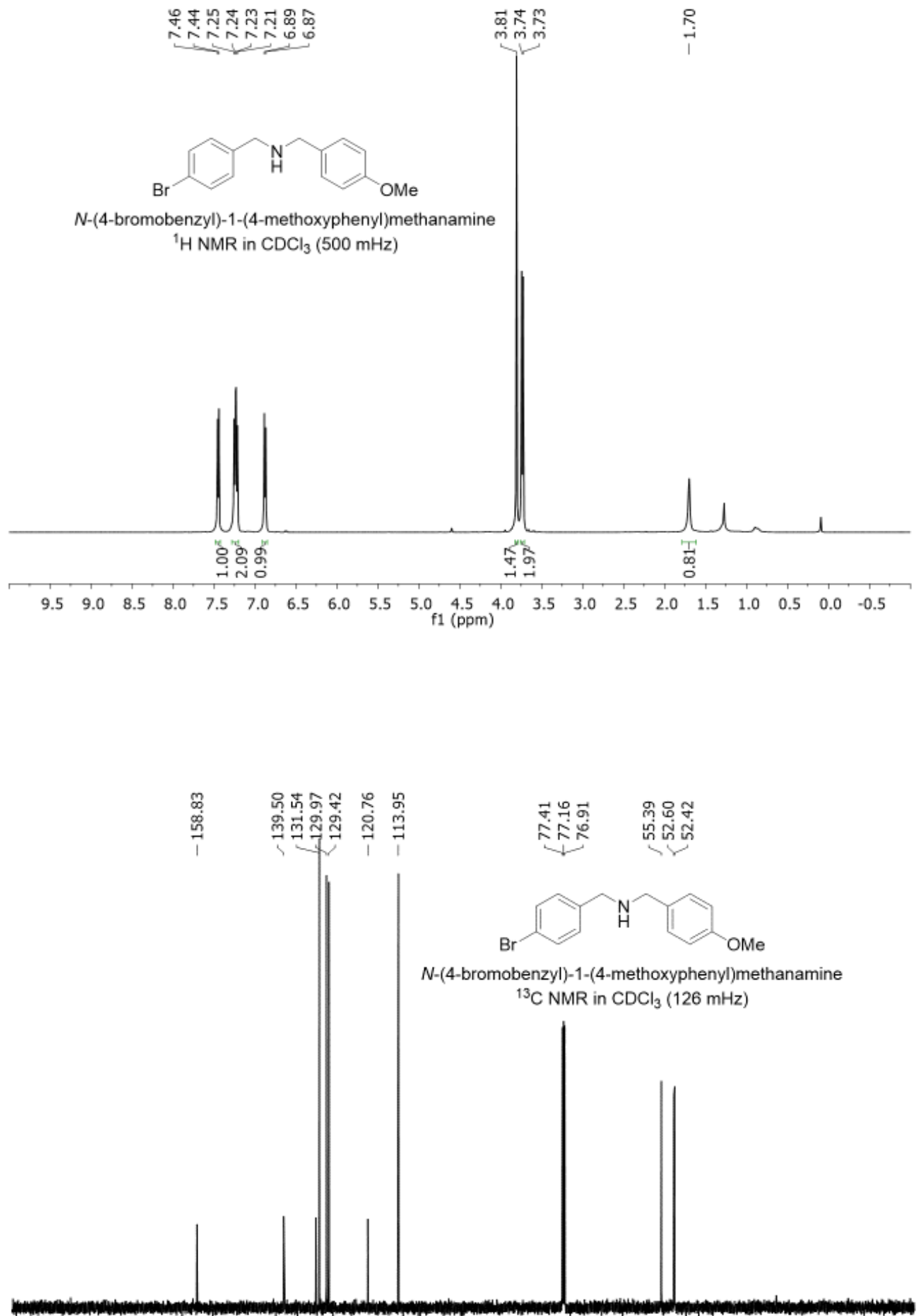

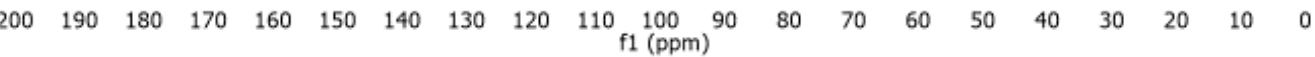



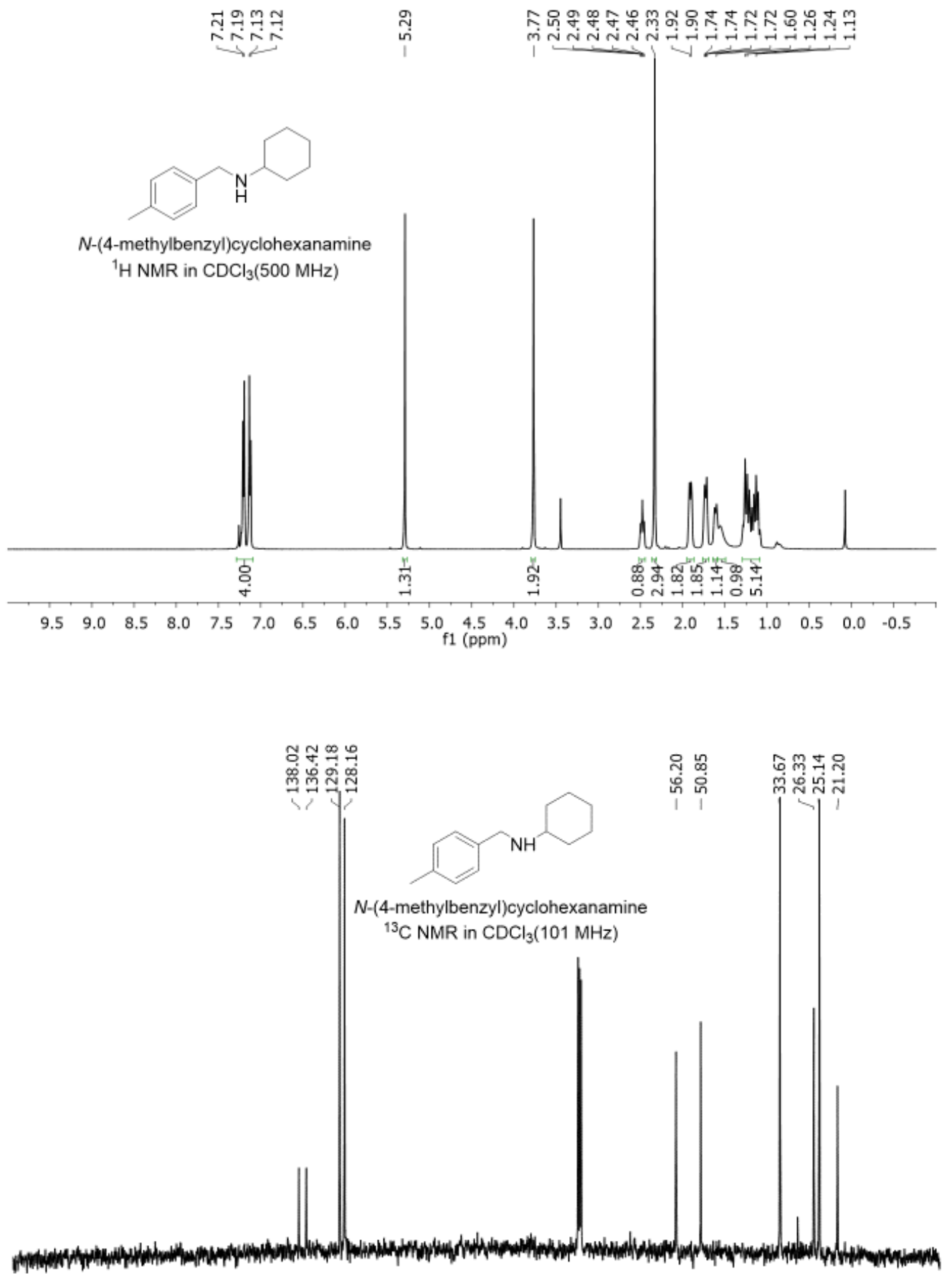

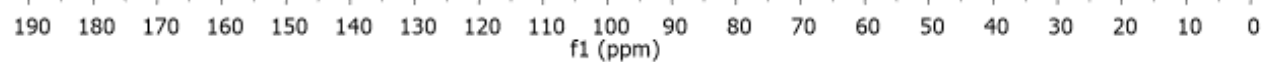




\section{References:}

1. (a) Jana, A.; Das, K.; Kundu, A.; Thorve, P. R.; Adhikari, D.; Maji, B., A Phosphine-Free Manganese Catalyst Enables Stereoselective Synthesis of $(1+n)$-Membered Cycloalkanes from Methyl Ketones and 1,n-Diols. ACS Catal. 2020, 10, 2615-2626. (b) Das, K.; Kumar, A.; Jana, A.; Maji, B., Synthesis and characterization of $\mathrm{N}, \mathrm{N}$-chelate manganese complexes and applications in $\mathrm{C} \mathrm{N}$ coupling reactions. Inorganica Chim. Acta 2020, 502, 119358.

2. Koval, Iryna A.; Huisman, M.; Stassen, Arno F.; Gamez, P.; Roubeau, O.; Belle, C.; Pierre, J.-L.; Saint-Aman, E.; Lüken, M.; Krebs, B.; Lutz, M.; Spek, Anthony L.; Reedijk, J., Dinuclear CuII Complexes with a New Phenol-Based Ligand Bearing Pyridine and Thiophene Substituents: Synthesis, Characterization and Interaction with Catechol Substrates. Eur. J. Inorg. Chem. 2004, 2004, 4036-4045.

3. Song, X.; Lim, M. H.; Mohamed, D. K. B.; Wong, S. M.; Zhao, J.; Hor, T. S. A., Re(I) carbonyl complexes containing pyridyl-imine and amine ligands: Synthesis, characterization and their catalytic olefin epoxidation activities. J. Organomet. Chem 2016, 814, 1-7.

4. (a) Gagare, P. V. R. a. P. D., Preparation of Ammonia Borane in High Yield and Purity, Methanolysis, and Regeneration. Inorg. Chem. 2007, 46 7810-7817. (b) Vencislav M. Parvanov, G. K. S., Nancy J. Hess, Luke L. Daemen, Monika Hartl, Ashley C. Stowe, Donald M. Camaionia and Tom Autrey, Materials for hydrogen storage: structure and dynamics of borane ammonia complex. Dalton Trans. 2008, $4514-4522$.

5. Hou, S.-F.; Chen, J.-Y.; Xue, M.; Jia, M.; Zhai, X.; Liao, R.-Z.; Tung, C.-H.; Wang, W., Cooperative Molybdenum-Thiolate Reactivity for Transfer Hydrogenation of Nitriles. ACS Catal. 2019, 10, 380-390.

6. Mukherjee, A.; Srimani, D.; Chakraborty, S.; Ben-David, Y.; Milstein, D., Selective Hydrogenation of Nitriles to Primary Amines Catalyzed by a Cobalt Pincer Complex. J. Am. Chem. Soc. 2015, 137, 88888891.

7. Shao, Z.; Fu, S.; Wei, M.; Zhou, S.; Liu, Q., Mild and Selective Cobalt-Catalyzed Chemodivergent Transfer Hydrogenation of Nitriles. Angew. Chem. Int. Ed. 2016, 55, 14653-14657.

8. $\quad$ Chen, F.; Topf, C.; Radnik, J.; Kreyenschulte, C.; Lund, H.; Schneider, M.; Surkus, A. E.; He, L.; Junge, K.; Beller, M., Stable and Inert Cobalt Catalysts for Highly Selective and Practical Hydrogenation of C identical withN and C horizontal lineO Bonds. J. Am. Chem. Soc. 2016, 138, 8781-8788.

9. Lu, Z.; Williams, T. J., A dual site catalyst for mild, selective nitrile reduction. Chem. Commun. 2014, 50, 5391-5393.

10. Szostak, M.; Sautier, B.; Spain, M.; Procter, D. J., Electron transfer reduction of nitriles using SmI2-Et3N-H2O: synthetic utility and mechanism. Org. Lett. 2014, 16, 1092-1095.

11. Lu, Z.; Williams, T. J., A dual site catalyst for mild, selective nitrile reduction. Chem. Commun. 2014, 50, 5391-5393.

12. Saito, Y.; Ishitani, H.; Kobayashi, S., Catalytic Hydrogenation of Aliphatic Nitro Compounds with Polysilane/Bone Charcoal-Supported Palladium Catalysts under Continuous-Flow Conditions. Asian J. Org.Chem. 2016, 5, 1124-1127.

13. Mercer, S. M.; Robert, T.; Dixon, D. V.; Chen, C.-S.; Ghoshouni, Z.; Harjani, J. R.; Jahangiri, S.; Peslherbe, G. H.; Jessop, P. G., Design, synthesis, and solution behaviour of small polyamines as switchable water additives. Green Chem. 2012, 14, 832.

14. (a) Bagal, D. B.; Bhanage, B. M., Recent Advances in Transition Metal-Catalyzed Hydrogenation of Nitriles. Adv. Synth. Catal. 2015, 357, 883-900. (b) Richard V. Jones, L. G., Norbert Varga, Daniel Szalay, Laszlo Urge, and Ferenc Darvas, Continuous-Flow High Pressure Hydrogenation Reactor for Optimization and High-Throughput Synthesis. J. Comb. Chem. 2006, 8, 110-116.

15. Lange, S.; Elangovan, S.; Cordes, C.; Spannenberg, A.; Jiao, H.; Junge, H.; Bachmann, S.; Scalone, M.; Topf, C.; Junge, K.; Beller, M., Selective catalytic hydrogenation of nitriles to primary amines using iron pincer complexes. Catal. Sci. Technol. 2016, 6, 4768-4772.

16. Sharma, D. M.; Punji, B., Selective Synthesis of Secondary Amines from Nitriles by a UserFriendly Cobalt Catalyst. Adv. Synth. Catal. 2019, 361, 3930-3936. 
17. Abonia, R.; Castillo, J. C.; Garay, A.; Insuasty, B.; Quiroga, J.; Nogueras, M.; Cobo, J.; D'Vries, R., A facile synthesis of stable $\beta$-amino- $\mathrm{N}$-/ O -hemiacetals through a catalyst-free three-component Mannich-type reaction. Tetrahedron Lett. 2017, 58, 1490-1494.

18. Singh, A.; Mobin, S. M.; Mathur, P., Preparation of the Ru3(CO)8-pyridine-alcohol cluster and its use for the selective catalytic transformation of primary to secondary amines. Dalton Trans. 2018, 47, 14033-14040.

19. Chung, H.; Chung, Y. K., Cobalt-Rhodium Heterobimetallic Nanoparticle-Catalyzed N-Alkylation of Amines with Alcohols to Secondary and Tertiary Amines. J. Org. Chem. 2018, 83, 8533-8542.

20. M. J. Frisch, G. W. T., H. B. Schlegel, G. E. Scuseria,M. A. Robb, J. R. Cheeseman, G. Scalmani, V. Barone, B. Mennucci, G. A. Petersson, H. Nakatsuji, M. Caricato, X. Li, H. P. Hratchian,; A. F. Izmaylov, J. B., G. Zheng, J. L. Sonnenberg, M. Hada,; M. Ehara, K. T., R. Fukuda, J. Hasegawa, M. Ishida, T. Nakajima,; Y. Honda, O. K., H. Nakai, T. Vreven, J. A. Montgomery, Jr.,; J. E. Peralta, F. O., M. Bearpark, J. J. Heyd, E. Brothers,; K. N. Kudin, V. N. S., T. Keith, R. Kobayashi, J. Normand; K. Raghavachari, A. R., J. C. Burant, S. S. Iyengar, J. Tomasi,; M. Cossi, N. R., J. M. Millam, M. Klene, J. E. Knox, J. B. Cross,; V. Bakken, C. A., J. Jaramillo, R. Gomperts, R. E. Stratmann,; O. Yazyev, A. J. A., R. Cammi, C. Pomelli, J. W. Ochterski,; R. L. Martin, K. M., V. G. Zakrzewski, G. A. Voth,; P. Salvador, J. J. D., S. Dapprich, A. D. Daniels,; O. Farkas, J. B. F., J. V. Ortiz, J. Cioslowski,; Fox, a. D. J., Gaussian, Inc., Wallingford CT. 2010.

21. (a) D., B. A., Density-functional thermochemistry. III. The role of exact exchange. J. Chem. Phys. 1993, 98, 5648. (b) Lee, C.; Yang, W.; Parr, R. G., Development of the Colle-Salvetti correlation-energy formula into a functional of the electron density. Phys. Rev. B Condens. Matter 1988, 37, 785-789.

22. (a) Hay, P. J.; Wadt, W. R., Ab initio effective core potentials for molecular calculations. Potentials for the transition metal atoms Sc to Hg. J. Chem. Phys. 1985, 82, 270. (b) Wadt, W. R.; Hay, P. J., Ab initio effective core potentials for molecular calculations. Potentials for main group elements Na to Bi. J. Chem. Phys. 1985, 82, 284-298.

23. Weigend, F.; Ahlrichs, R., Balanced basis sets of split valence, triple zeta valence and quadruple zeta valence quality for $\mathrm{H}$ to Rn: Design and assessment of accuracy. Phys. Chem. Chem. Phys. 2005, 7, 3297-305.

24. (a) Tomasi, J.; Mennucci, B.; Cammi, R., Quantum Mechanical Continuum Solvation Models. Chem. Rev. 2005, 105, 2999-3093. (b) Cossi, M.; Barone, V.; Cammi, R.; Tomasi, J., Ab initio study of solvated molecules: a new implementation of the polarizable continuum model. Chem. Phys. Lett. 1996, 255, 327-335. (c) Cancès, E.; Mennucci, B.; Tomasi, J., A new integral equation formalism for the polarizable continuum model: Theoretical background and applications to isotropic and anisotropic dielectrics. J. Chem. Phys. 1997, 107, 3032. (d) Barone, V.; Cossi, M., Quantum Calculation of Molecular Energies and Energy Gradients in Solution by a Conductor Solvent Model. J. Phys. Chem. A 1998, 102, 1995-2001. 